
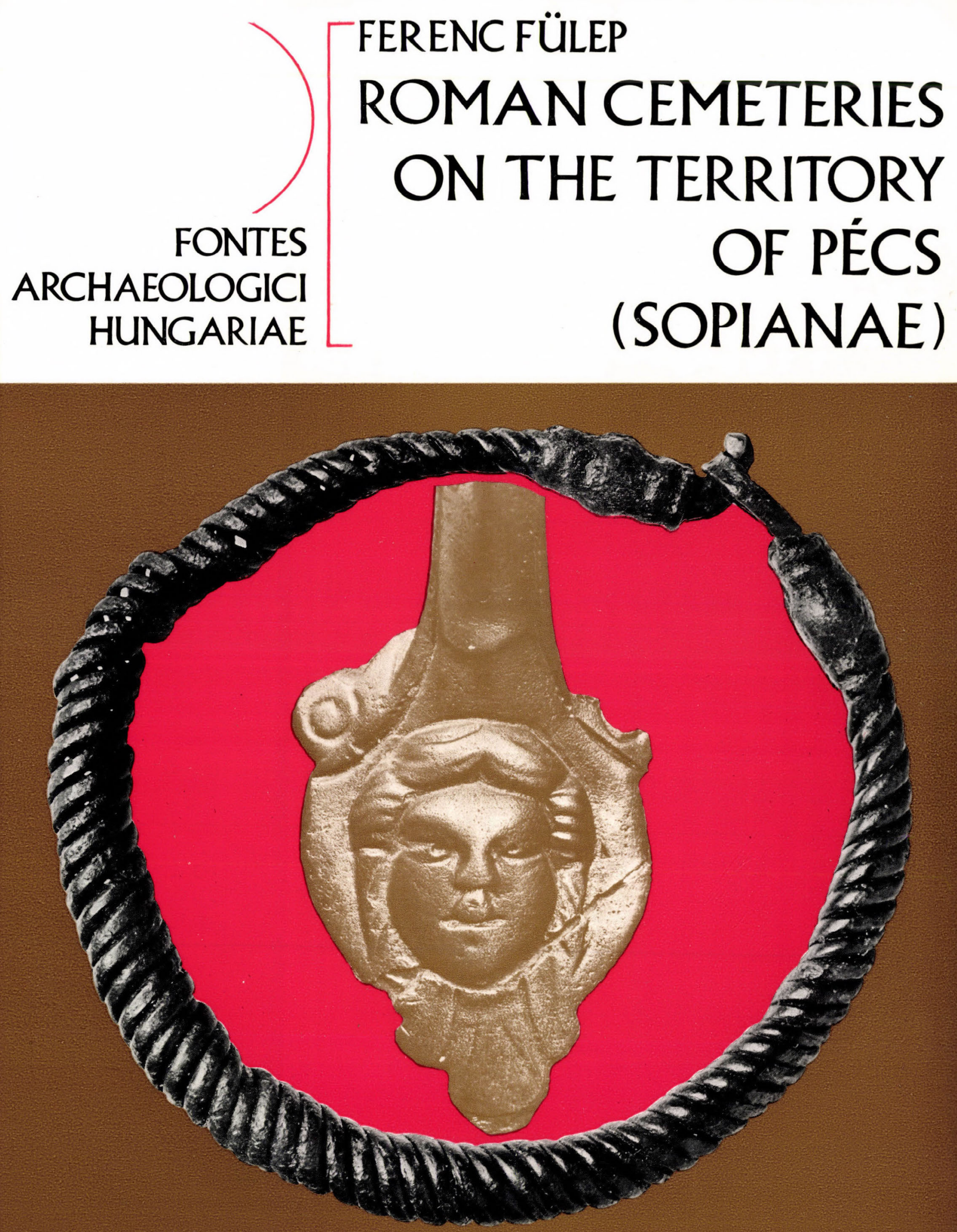


\section{FERENC FÜLEP}

\section{ROMAN CEMETERIES \\ ON THE TERRITORY OF PÉCS (SOPIANAE)}

\section{FONTES ARCHAEOLOGICI HUNGARIAE}

More than 20 years of excavations and research work have enabled the author to publish the material of the Roman cemeteries found on the territory of the Roman town Sopianae - the central part of the present town of Pécs. From the first graves discovered around 1716 to the author's own latest excavation results, we follow his reconstruction work during the course of which more than 250 earlier discovered graves were identified. The history of the Roman town is based on this thorough research work. The publication of this material is of great benefit to history in general apart from local and national history.

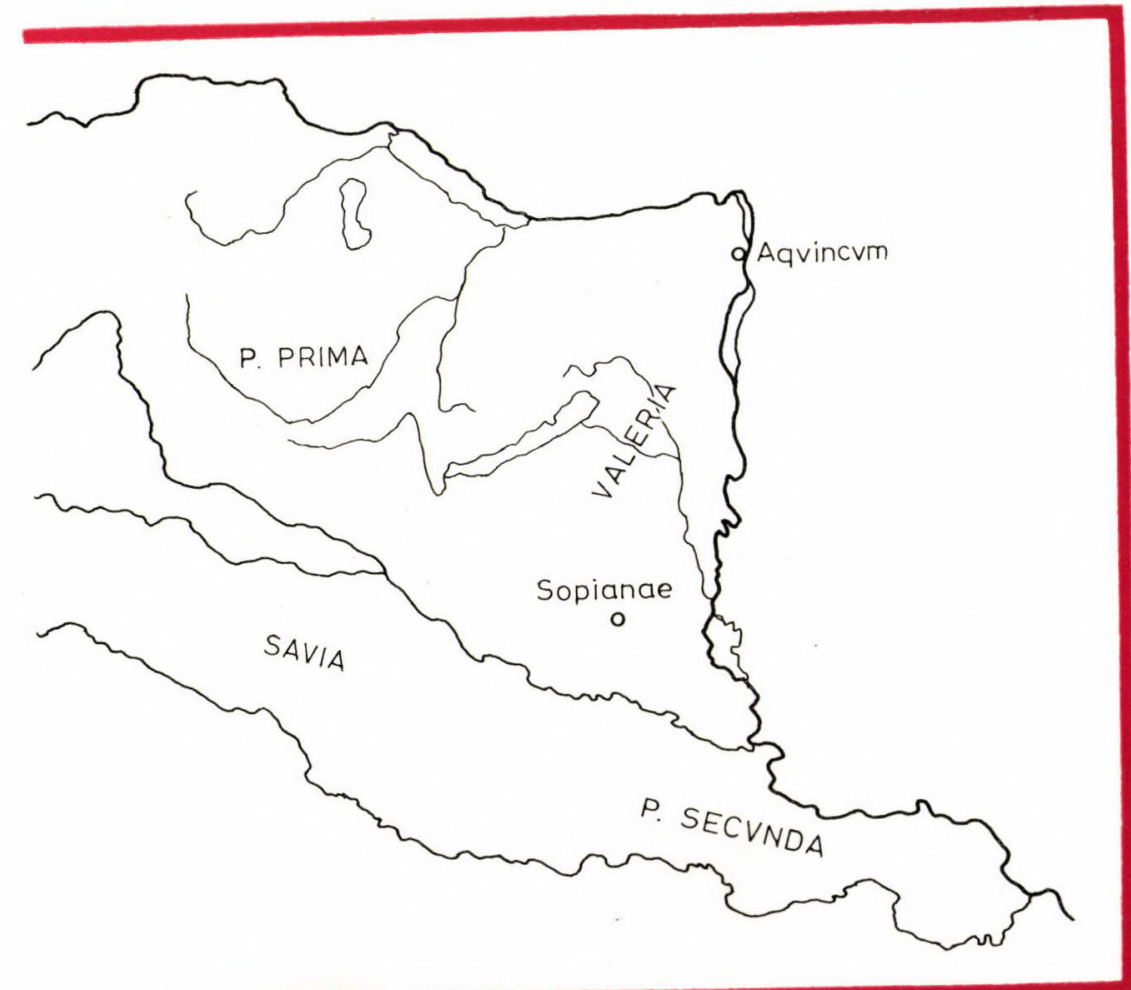


ROMAN CEMETERIES ON THE TERRITORY OF PÉCS (SOPIANAE) 


\section{FONTES ARCHAEOLOGICI HUNGARIAE}

ADIUVANTIBUS

L. CASTIGLIONE • L. GEREVICH - I. KOVRIG • GY. LÁSZLÓ

A. MÓCSY • E. PATEK

REDIGIT

I. HOLL

AEDES ACADEMIAE SCIENTIARUM HUNGARICAE BUDAPESTINI MCMLXXVII 
FERENC FÜLEP

\section{ROMAN CEMETERIES ON THE TERRITORY OF PÉCS (SOPIANAE)}

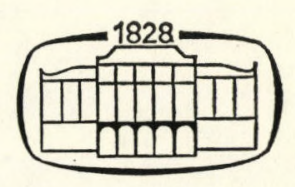

AKADÉMIAI KIADÓ • BUDAPEST 1977 
Translated by

VERA GÁTHY

ISBN 9630510790

(C) Akadémiai Kiadó, Budapest 1977

Printed in Hungary 


\section{CONTENTS}

$\begin{array}{ll}\text { Introduction } & 7\end{array}$

I. Historical outline 9

II. Burials around the present cathedral 11

III. Burials in other parts of the present town 23

IV. Burials in the suburban area

V. Articles from unspecified sites $\quad 57$

Abbreviations $\quad 58$

Changed street names in Pécs $\quad 60$

$\begin{array}{ll}\text { Bibliography } & 61\end{array}$

Index $\quad 63$

Plates $\quad 65$ 



\section{INTRODUCTION}

This work presents the material recovered since 1716 from the cemeteries of Roman date on the territory of Pécs (Sopianae). From that year onwards graves belonging to the Roman cemeteries, which are situated in the northern and northeastern part of the present city, have been brought to light by various construction and drainage works, and occasionally by excavations. Unfortunately, with the exception of the drawings of Ottó Szőnyi and Gyula Gosztonyi, no maps of the earlier excavations of the cemeteries have come down to us, and the situation has been further aggravated by the fact that the finds from the graves were not kept separate from one another; in 1939 the whole of the material was entered in an inventory irrespective of the relationship between the various objects, and in most cases those compiling it had not the slightest idea of the conditions under which the graves or their contents had been found.

In many cases the identification of the graves was the result of a long work of reconstruction. For this task, four volumes of the acquisitions register of the Pécs Municipal Museum, which were believed lost but have somehow been recovered in the Janus Pannonius Museum of Pécs, were of great help, as all of them contained valuable information relating to the graves and necessary for the identification of individual finds. We have also gone through the news reports in the local press, which were in many cases our only guides to the excavations, since no diary was kept on the spot in those early days.

We have also found valuable information in the Archives Departments of the Janus Pannonius Museum of Pécs and the Hungarian National Museum. By reconstruction in this way we were able to identify about 250 graves more or less accurately.

To the reconstructed graves we added the new ones we ourselves had excavated, and we fitted them into their appropriate place in the layout of the cemetery. With the exception of two major groups of burials (Geisler Eta Street 8 and István Square 12-Geisler Eta Street 14), the graves excavated by us have also been included in the present work. Of these two groups, one was published in the Archaeologiai Értesíto" (96/1969/ pp. 3 f.), and the other will be published in the monograph on Sopianae.

We had in addition to establish a method for numbering the graves, because almost all the sources used had their own individual systems for the graves already excavated. New numbers were therefore given to all the burial-chambers and graves, while the earlier numbering of the different authors has of course been preserved in the corresponding references or notes. On our plan of the cathedral area the burial-chambers and graves found there appear from the beginning with new numbers. During the repeated and thorough scrutiny of the interior of the burial-chambers several graves were found which had hitherto escaped attention.

A brief outline of the development and history of the cemeteries is given immediately below. A detailed historical survey and the place of the cemeteries in the historical context of the Roman town will be found in the monograph now ready to go to press.

Next we describe the various parts of the cemetery, starting from the groups of graves in the vicinity of the cathedral. Subsequently a separate chapter is devoted to the groups of graves known in other parts of the town, following, wherever possible, a topographical order. In the description of the cemetery a number of such groups are mentioned which were found in the course of almost two decades of excavations conducted by us. In presenting this volume it has been our intention to make available the rich material of the cemeteries of Pécs as a source for further research.

I would like to thank Mr J. Karáth and Miss K. Nádor for the photos; Mr E. Egyed, Mrs K. P. Mózes and A. Semsey for the drawings. 


\section{HISTORICAL OUTLINE}

The town of Sopianae, situated in the angle of the rivers Danube and Dráva in the south-west of Pannonia, was founded during the early or mid 2nd century AD. This is shown both by earlier and by recent research ${ }^{1}$ (Suppls 1-2). Although written records have not survived, it may be supposed that it was elevated to the rank of municipium as early as the reign of Hadrian. During the second half of the second century, under Marcus Aurelius, the wars against the Quadi, Marcomani and Sarmatians resulted in the destruction of the town, which was only gradually rebuilt during the third century.

Neither earlier nor recent research has revealed any material evidence in the environs of the Roman town to indicate the existence there of a cremation cemetery in the second century. The only such cemetery which could definitely be identified is to the north of the town at Pécs-Vasas. ${ }^{1 a}$ The excavations revealed nothing to suggest that any part of the Roman town had been built on an earlier cremation cemetery.

Only rather scanty evidence is available for the location of cremation burials. At Széchenyi Square, for instance, on the ground of the parish church, there may have been a columbarium in the cemetery; a knee brooch was found in grave $\mathrm{R} / 207$; a furrowed urn together with a badly burned second century brooch was found in graves R/278-279 at the brick-yard of Mohácsi Street; and finally we consider as indirect evidence the number of urns found in grave $\mathrm{R} / 18$, which may have come from the cremation cemetery cleared out during the period of inhumation. One of the important private collections of Pécs, the Juhász collection, contains urns found in the "inner city". The cremation ground (ustrinum) found in Rákóczi Street belongs to a cremation cemetery of the fourth century and cannot be related to the cremation cemeteries of the early imperial period. As hitherto no such cemeteries had been found, early glasses are almost entirely lacking from the town.

Inhumation burials appeared in the third century at present-day Sallai Street, considered to be the northern boundary of the Roman town. The third century cemetery spread in two directions: north of Sallai Street to the line of present Geisler Eta (Apáca) Street, and to Széchenyi Square in a north-easterly direction. The majority of them are brick graves and were richly furnished with grave-goods: generally men were buried in their military garments and women were interred with their jewels. From another private collection of Pécs, the Horváth collection, the Hungarian National Museum has obtained a rich assemblage of third century jewels which, in our view, were found in the third century graves towards the end of the last century. The orientation of the graves is E-W with the head to the east.

These rich grave-goods provide extremely important evidence for the third century revival of the town, which was again disturbed by an attack, presumably of the Roxolani, around 260-270 A.D.

Towards the end of the third century a decisive change took place in the life of the town, when after 293, under Diocletian, Sopianae became a seat of civil administration in Valeria Province and the headquarters of the praeses was shifted from Aquincum to Sopianae. ${ }^{2}$ Material evidence of the settlement of Carpi in the town, mentioned by Ammianus Marcellinus, could not, however, be identified. After its establishment as capital of the Province, largescale construction was carried out in the town at the end of the third century and during the fourth, and the increase in the volume of finds, together with their obvious richness, indicates that there was considerable material and intellectual prosper-

1 See in detail: Fülep $V$.

$1 a$ F. Fülep: Das frühkaiserzeit]iche Gräberfeld von Vasas, Acta Arch. Hung. 9(1959) p. 371 and supra.

2 Amm. Marc. 28, 1. 5. 
ity in the town. This is reflected in the large quantity of finds, big buildings, and burial structures of artistic value which are unique in the province.

The spread of Christianity after 313 obviously extended to regions north of the Dráva, with the result that in the fourth century Sopianae became one of the important centres of Christianity. During the first decades of the fourth century early Christian burials began to appear in the town. The alignment of the graves became W-E without exception, with the head to the west. On the basis of the development of the third-century cemetery it may be deduced that the early Christian burials spread from the south to the north, directly adjoining the third century cemetery, and mounting the hillside right up to the present-day cathedral. To the NE it spread as far as the eastern edge of Széchenyi Square, or even further, and its eastern limits should also be looked for along the eastern side of Széchenyi Square.

The central group of the early Christian cemetery at Széchenyi Square and Geisler Eta Street, situated in the vicinity of the cathedral, was surrounded by graves of $\mathbf{E}-\mathrm{W}$ orientation (head to the east) from the south as far as Geisler Eta (Apáca) Street. Along the northern side of Geisler Eta Street we succeeded in establishing the line where the earlier graves of $\mathbf{E}-\mathrm{W}$ orientation, head to the east, met the ones of W-E alignment (head to the west), which were burials in accordance with the early Christian rite. We also found these earlier graves representing the transition at burial-chambers II and VII (Graves R/32, R/40, heads to the east).

During the middle or the second half of the fourth century, such graves appeared in the northern and north-eastern part of the cemetery with the early Christian characteristics (Székesfehérvár Street, Széchenyi Square-Parish Church, Regős Street, Gründler Street); here, besides the W-E orientation, a different alignment of the graves can also be recognized (Széchenyi Square, Parish Church) and in contrast with the graves of W-E orientation where associated coins are only exceptional, we found in these graves a number of coins and other objects. Men were buried in their military garments with belt fittings, there were iron knives and tools in the graves, and the women were ornamented with jewels. According to our assessment this burial custom continued in use until the end of the fourth century, parallel with the early Christian rites.

Two features are characteristic of the early Christian burials:

(a) the small number, or almost entire absence of coins,

(b) the marked decrease in the number of other associated objects; there is hardly any pottery in the graves, although the quantity of glassware sharply increased.

The circulation of coins was undisturbed in Sopianae until 378 when the inflow of new issues ceased. The settlement of "foederati" peoples, Huns, Germans and Alani in the vicinity of the town in the 380 s may account for this (AlatheusSaphrac). ${ }^{3}$ At that time the town must have been in a rather dilapidated condition, as indicated by the finds of graves among the Roman buildings at the Post Office; the cemetery had therefore penetrated into the town by the end of the fourth century. Information derived from the burials show that life continued in the town to the end of the fourth century and even to the beginning of the fifth.
${ }^{3}$ Zos. IV; Jord. Get. 139-142; A. Alföldi: Der Untergang der Römerherrschaft in Pannonien I-II, Berlin 1924-26, Vol. II, p. 67; R. Egger: Der Heilige Herma- goras. Carinthia I. 2. Teil. Jahrgang 136-138, Klagenfurt 1948, p. 219; A. Mócsy: Pannonia. PWRE Suppl. Bd. IX. Stuttgart 1962. p. 578. 


\section{BURIALS AROUND THE PRESENT CATHEDRAL}

\section{BURIAL-CHAMBER I, WITH WALL PAINTINGS $(P l .50)$}

This was discovered in 1780 on the southern side of the present cathedral. ${ }^{4}$ On the basis of our reconstruction there was a huge tomb inside, in front of its northern wall (Grave I/1). W-E orientation.

In 178013 brick graves $(P l .50)$ were found on the site of the Archive of the Chapter House. ${ }^{5}$ Apparently these were uncovered by J. Koller. ${ }^{6}$ Gy. Gosztonyi only gives an approximate location for the graves.? The depth and orientation of the graves are not known either.

Grave R/1. A drawing of this grave was published by J. Koller. ${ }^{8}$ L: ca. 200 , W: $65 \mathrm{~cm}$. Its roof was made of four pairs of brick: $\wedge$. On each of two triangular gable bricks, one at the head and one at the foot, there was a painted monogram of Christ. In it there was an intact skeleton, but its orientation is not indicated.

Graves R/2-13. No notes are extant on these graves. According to J. Koller the graves contained fragments of one small glass unguent bottle and a bigger globular glass flask. ${ }^{9}$

\section{BURIAL - CHAMBER II, WITH WALL PAINTINGS $(P l .50)$}

This was found in 1939 in front of the main entrance of the cathedral. ${ }^{10}$ Originally there was

\footnotetext{
${ }^{4}$ Entry DACL XIII. Pannonie, p. 1058. Dates its finding wrongly to 1870 ; Kádár $I$, p. 4 and supra; Gosztonyi $I$, p. 93 and supra, drawing 6 ; Kádár $I I$, p. 65 and supra; Gosztonyi $I V$, p. 16 and supra, Fig. 3, 1-3, drawings 6-8; Fülep III, p. 151, note 4 for further literature.

${ }^{5}$ Koller, p. 33, Tab. XIV. Figs 7-9; Baranya $I I$, p. 452; Szönyi I, p. 19; Nagy, p. 36; Gosztonyi I, p. 105.

${ }^{6}$ Koller, Tab. XIV, Fig. 7.

${ }^{7}$ Gosztonyi I, p. 85, drawing 5, circle b.

${ }^{8}$ Koller, ibid.
}

one grave in it and later on another was added (Graves II/1-2). W-E orientation. ${ }^{11}$

On the eastern side of the corridor leading to painted burial-chamber I, three graves were found:

Grave R/1412 (Pl. 50). L: 149, W: 38, outer W: $100 \mathrm{~cm}$. Height of its walls: $42-59 \mathrm{~cm}$. Its roof was constructed of five pairs of keyed bricks: $\wedge$. Both interior and exterior were plastered. W-E orientation. The skeleton was lying on a thin layer of sand and there were no associated objects. O. Szőnyi remarks that all these graves have a W-E orientation with the head always at the western end of the grave..$^{13}$

Grave R/15 ${ }^{14}(\mathrm{Pl} .50)$. South of the above grave. Inner L: 172, W: $45 \mathrm{~cm}$. Height of the side walls: 50, total height: $90 \mathrm{~cm}$. The western gable of the grave was made of quarry stone carved in triangular shape. The bottom of the grave: six bricks. At the head there was a small cushion made of brick, its height: $9 \mathrm{~cm}$. The interior of the grave was plastered.

Associated articles:

1. Bronze brooch. With three knobs and of crossbow type. Decoration consists of ribbed cordons at the base of the knobs and bow, and three pairs of punched ringlets. It is intact, but its pin is missing. H: 8.5, W: $5.1 \mathrm{~cm}$. (In the collection of the bishopric, without inventory number, $P l$. 1, No. 1.)

2. Iron knife. With one edge only. Its edge is badly corroded and it has a broken tang. L: 13.5, W: 1.5 , Th: $0.4 \mathrm{~cm}$ (see: $P l .1$, No. 5).

\footnotetext{
${ }^{9}$ Koller, Tab. XIV, Figs 8-9.

${ }^{10}$ Gosztonyi I, p. 104; Gosztonyi III, p. 196 and supra for further literature; Gosztonyi $I V$, p. 22 and supra, drawings $9-10$; Fülep $I V$, p. 91 and supra for further

11 Fülep $I V$, ibid.

12 O. Szőnyi, PBMÉ 6(1913) p. 121; Gosztonyi $I$, p. 109, Grave XLIV (corresponds to grave I of Möller)

13 O. Szőnyi, Dunántúl, 25 December, 1913.

14 Gosztonyi I, p. 109, Grave XLV (corresponds to
} literature. grave II of Möller). 
According to the description by O. Szőnyi, this grave contained a skull of curious shape, and a second skull, probably of a horse, was found in the crumbled earth. ${ }^{15}$

At the western end of the grave the roof was made of a double row of bricks whereas at the eastern end there was only a single row. The grave itself may have been opened at some time, allowing earth to get into it together with the skulls.

\section{BURIAL-CHAMBER III $(P l .50)$}

According to O. Szônyi this was found during the last century when the corridor leading to painted burial-chamber I was built. ${ }^{16}$

Grave $\mathbf{R} / \mathbf{1 6}^{17}(\mathrm{Pl}$. 50). This was built towards the north-east corner of burial-chamber III. Inner L: $161, W: 41.5 \mathrm{~cm}$. The grave becomes narrower towards the east. H. of wall: $49.5 \mathrm{~cm}$. Its roof is made of keyed bricks. A thin layer of sand covered the bottom of the grave. W-E orientation. Two skeletons were found side by side in the grave.

Grave $\mathbf{R} / \mathbf{1 7}^{18}(P l$. 50). This was found at the north-east corner of burial-chamber III. Inner L: 162, W: 55 at the E, $48 \mathrm{~cm}$ at the W. The wall of the grave was of two rows of brick at the $\mathrm{S}, \mathrm{N}$, and $\mathrm{W}$, while at the $\mathrm{E}$ it was of plastered masonry. Its roof was made of brick: $\wedge$. Below the head there was a small cushion made of plaster. W-E orientation. In the grave there was the skeleton of a young man. There were no associated objects.

Grave $\mathbf{R} / \mathbf{1 8}^{19}(P l$. 50). North of the above grave. Inner L: 178, inner W: 85 at the bottom, $59 \mathrm{~cm}$ at the roof; its side walls are therefore inclining. $\mathrm{H}$ : $87 \mathrm{~cm}$. The roof of the tomb is made of large bricks (four and a half pairs): $\Lambda$. There was a skeleton in the grave. W-E orientation, head to the west. According to the description by O. Szőnyi there are a strongly drawn cross and an anchor on two bricks from the grave. The bottom of the grave was covered with black sand, and its walls

15 O. Szőnyi, PBMÉ 6(1913) p. 123; Idem, Dunántúl 3 September, 1913.

${ }^{16}$ O. Szőnyi, Dunántúl, 25 December, 1913; Gosztonyi $I V$, p. 28, drawing $11,3 / \mathrm{a}-\mathrm{c}$.

17 O. Szőnyi, $P B M E ́$ 6(1913) p. 121 and supra; Gosztonyi $I$, p. 109, Grave XLVI (corresponds to grave III of Möller).

18 O. Szőnyi, ibid; Gosztonyi I, p. 109, Grave XLVII (corresponds to grave IV of Möller).

19 O. Szőnyi, PBMÉ 6(1913) p. 124; Nagy, p. 36; Gosztonyi I, p. 109, Grave XLVIII (corresponds to grave $\mathrm{V}$ of Möller). were plastered. ${ }^{20}$ From the earth fallen into the grave during excavation four pottery vessels came to light, and after removing the earth 45 vessels were uncovered at the western corner of the grave:

1. Pottery beaker. Grey, coarse. It has a coarse spot on its shoulder which stuck to it during firing, and a dark patch on its side. $\mathrm{H}$ : 12.7, DM: 9.6, DB: $5.2 \mathrm{~cm}$. (In the collection of the bishopric, without inventory number, $\mathrm{Pl}$. 2, No. 1.)

2. Pottery beaker. Grey, smooth, worn dark grey slip. H: 14, DM: 10, DB: $5.9 \mathrm{~cm}$ ( $P l .2$, No. 16).

3. Pottery beaker. Brownish-grey, somewhat coarse. H: 12.3, DM: 10, DB: $6 \mathrm{~cm}$ (Pl. 2, No. 3).

4. Pottery beaker. Reddish-brown, coarse, of clay mixed with pebbles. H: 12.4 , DM: 9.6 , DB: $5.2 \mathrm{~cm}$ (Pl. 2, No. 8).

5. Pottery beaker. Brownish-grey, coarse. Imprints were made on its side before firing. The shoulder of the vessel is ornamented with several small, vertical lines. $\mathrm{H}:$ 11.5, DM: 9.8, DB: $5.8 \mathrm{~cm}$ (Pl. 2, No. 5).

6. Pottery beaker. Brownish-grey, of clay mixed with white grit. At the neck there are indentations resulting from finger pressure. On its surface there are sheafs of burnished lines. H: 12, DM: 10.2, DB: $7.5 \mathrm{~cm}$ (Pl. 2, No. 6).

7. Pottery beaker. Grey, coarse; of clay mixed with grit and mica. The upper part of the vessel is ornamented with fine horizontal lines. H: 10.1, DM: 8.1, DB: $4.1 \mathrm{~cm}$ (Pl. 2, No. 7).

8. Pottery beaker. Light brown, coarse. Dark marks on its side. H: 12, DM: 7.7, DB: $4.1 \mathrm{~cm}$ (Pl. 2, No. 4).

9. Pottery beaker. Dark grey, coarse, rough surfaced, of clay mixed with white grit. With dull dark grey paint. H: 11.1, DM: 10, DB: $6.2 \mathrm{~cm}$ (II/1) ${ }^{21}$ ( $P l .4$, No. 1).

10. Pottery beaker. Grey, somewhat coarse. Patches of rough material on its side stuck to it during firing. H: 9.8, DM: 9.4, DB: $5.6 \mathrm{~cm}$ (Pl. 2, No. 10).

11. Pottery beaker. Grey, coarse; of clay mixed with white grit. Traces of worn black paint. H: 11.2, DM: 9, DB: $5.4 \mathrm{~cm}$ (Pl. 2, No. 11).

12. Pottery beaker. Grey, coarse. H: 10.5, DM: 10.3, DB: $5.4 \mathrm{~cm}$ (Pl. 2, No. 9).

20 NCM Archives, 690/1913. According to the minutes of 29 August and 1-2 September: ". . . the S side of the top of the grave had collapsed and as a result the grave was filled with earth. At the $\mathrm{S}$ end of the grave there were two vertical bricks practically supporting the earth above the $\mathrm{S}$ roof. On one of them there was the anchor and on the other one the cross. The 45 vessels were lying in the W end of the grave."

${ }^{21}$ In some of the vessels there were slips of paper with Szőnyi's handwriting. The meaning of the numbers on them could not be deciphered hitherto. 
13. Pottery beaker. Grey, somewhat coarse. With light and dark grey spots. H: 10.5, DM: 8.5 , DB: $4.2 \mathrm{~cm}(\mathrm{II} / 5)^{22}$ (Pl. 4, No. 2).

14. Pottery flagon. Cream-coloured, of gritty clay. Wide ribbon handle. There is a wide stripe of brownish-red paint on the shoulder of the vessel. H: 27.1, DM: 8, DB: $9 \mathrm{~cm}(P l .5$, No. 5).

15. Jar. Dark grey. Its mouth is irregular, of oval shape. It is ornamented with a band of impressed oblique lines running in a continuous spiral around it. On its shoulder, rough V-shaped lines are smoothed in and the adjacent area of the sides is ornamented with a smoothed $\mathrm{O}$-shaped pattern. H: 20.8, DM: 15.4, 13.3, DB: $8.5 \mathrm{~cm}$ (Pl. 3, No. 1; Pl. 9, No. 1).

16. Jar. Neutral-coloured, thick walled. With treble ribbed handle. It has a wide stripe of reddishbrown paint. H: 21, DM: 15.4, DB: $8 \mathrm{~cm}(P l .3$, No. 6 ; Pl. 7, No. 3 ).

17. Pottery beaker. Brownish-grey, smooth, the upper part of the vessel with greyish-brown, shiny glaze. H: 12.2, DM: 10.7, DB: $5.6 \mathrm{~cm} \mathrm{(Pl.} \mathrm{2,}$ No. 12).

18. Pottery beaker. Grey, smooth surface. H: 10.5, DM: 7, DB: $4.5 \mathrm{~cm}$ (VII/2) $)^{23}$ (Pl. 4, No. 12).

19. Two-handled pottery vessel. Made of neutralcoloured clay. With brown, metallic glaze. H: 13.4, DM: 14, DB: $7.6 \mathrm{~cm}$ (Pl. 6, No. 4; Pl. \%, No. 1).

20. Pottery beaker. Grey, coarse, of rough-surfaced clay mixed with pebbles. Rough workmanship. H: 12.9, DM: 6.3, DB: $10.2 \mathrm{~cm}$ ( $P l .2$, No. 13).

21. Pottery beaker. Grey, somewhat smooth surface, of clay mixed with grit. Traces of badly worn black glaze. H: $14, \mathrm{DM}: 6, \mathrm{DB}: 9.6 \mathrm{~cm}$ (Pl. 2, No. 14).

22. Two-handled pottery vessel. Brick-coloured, somewhat rough. With two narrow handles. H: 18.4, DM: 6.2, DB: $9.1 \mathrm{~cm}$ (Pl. 6, No. 1).

23. Two-handled pottery vessel. Yellowish-pink. With badly worn red glaze. H: 14, DM: 14.5, DB: $7.5 \mathrm{~cm}$ ( $P l .6$, No. 3; Pl. 7, No. 2).

24. Two-handled pottery vessel. Of red clay, somewhat rough. With worn, spotted black paint. Inside there is a black encrustation, presumably of organic matter. H: 13.9, DM: 11, DB: $6 \mathrm{~cm}$ (Pl. 6, No. 2).

25. Pottery beaker with indented sides. Brownishgrey, smooth surface. With five round shaped indentations and shiny brownish-grey glaze. H: 13.3, DM: 9.3, DB: $6.2 \mathrm{~cm}$ (Pl. 3, No. 2).
26. Beaker with handle, of grey fabric. With double ribbed handle. H: 10.4, DM: 9, DB: $4.8 \mathrm{~cm}$ (Pl. 4, No. 3).

27. Pottery beaker with handle. Grey, rough-surfaced. H: 11.9, DM: 9.3, DB: $5.6 \mathrm{~cm}$ (Pl. 5, No. 2).

28. Urn. Grey clay, rough-surfaced. H: $22.5 \mathrm{~cm}$, DM: 9, DB: $8.8 \mathrm{~cm}$ (Pl. 3, No. 5; Pl. 9, No. 2).

29. Pottery flagon. Brick-coloured, with wide ribbon handle. Wide bright red painted stripe. H: 24.5, DM: 6.8, DB: $8.7 \mathrm{~cm}(\mathrm{~V} / 2)^{24}(P l$. 4, No. 4).

30. Pottery jug. Brick-coloured, thick walled. With a wide stripe of brownish-red paint. H: 21, DM: 6.9, DB: $6.8 \mathrm{~cm}$ (Pl. 5, No. 6).

31. Bottle-shaped pottery flagon. Two-handled. Light grey, somewhat rough-surfaced. H: 28.2, DM:

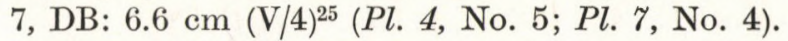

32. Glazed jug. Brick-coloured, rough. With shiny brownish-green lead glaze. H: 21.4, DM: 6.6 DB: $5.3 \mathrm{~cm}(\mathrm{~V} / 5)^{26}(P l .4$, No. 6; Pl. 9, No. 3).

33. Pottery jug. Grey, rough-surfaced. With double ribbed handle. H: 17.3 , DM: $7.6, \mathrm{DB}: 4.9 \mathrm{~cm}$ (Pl. 5, No. 4).

34. Pottery jug. Brownish-grey, of somewhat gritty clay, rough. With clover-shaped lip and badly worn dark grey paint. H: 16, DM: 7.5, DB: $6 \mathrm{~cm}$ $(\mathrm{V} / 7)^{27}$. (Pl. 4, No. 7; Pl. 9, No. 4).

35. Pottery beaker. Grey, somewhat rough. Smoothed areas on its surface. H: 12.8, DM: 9,6, DB: $5.5 \mathrm{~cm}$ (Pl. 4, No. 8).

36. Pottery bowl. Grey, smoothed surface. Traces of burnishing on its surface. H: 9.2, DM: 14.4, DB: $6.5 \mathrm{~cm}$ ( $P l .3$, No. 4).

37. Pottery beaker. Grey, rough. Worn surface with black paint. H: 13.4, DM: 9.2, DB: $6 \mathrm{~cm}$ (Pl. 2, No. 2).

38. Pottery beaker. Made of fine red clay. With badly worn red paint. H: 7.8, DM: $5.6, \mathrm{DB}: 2.9 \mathrm{~cm}$ (Pl. 3, No. 3).

39. Stove tile. From the time of Turkish occupation (16-17th centuries A. D.) (Pl. 4, No. X).

40. Pottery flagon. Brownish clay. Shiny dark brown, spotted surface, with light brown paint. $\mathrm{H}$ : 18.3, DM: 2.7, DB: $18 \mathrm{~cm}$ (Pl. 5. No. 7; Pl. 6, No. 5).

41. Pottery flagon. Brick-coloured, smooth surface. With smeared brownish paint. H: 18.5, DM: 3, DB: $6 \mathrm{~cm}$ (Pl. 5, No. 8; Pl. 6, No. 6).

42. Pottery vessel. Cream-coloured, of fine fabric. With worn brownish-red paint. H: 17.5, DM: 7, DB: $6.4 \mathrm{~cm}$ (Pl. 4, No. $9 ; P l .8$, No. 2).

43. Pottery vessel. Cream-coloured, somewhat rough. With worn reddish-brown paint. H: 20.8,

25 Cf. Note 21.

26 Cf. Note 21.

27 Cf. Note 21. 
DM: $9.5, \mathrm{DB}: 7 \mathrm{~cm}(\mathrm{VI} / 4)^{28}(P l .4$, No. $10 ; P l .8$, No. 1).

44. Pottery jug. Cream-coloured, somewhat rough. With clover-shaped lip (broken). The surface of the vessel is covered with a pattern, running down in a continuous spiral band, consisting of small lines. H: 29, DB: $8.2 \mathrm{~cm}(P l .5$, No. 10; Pl. 8, No. 4).

45. Pottery jug. Cream-coloured, somewhat rough. With clover-shaped lip. Decoration as above. H: 18, DB: $7.8 \mathrm{~cm}$ (Pl. 5, No. 9; Pl. 8, No. 3).

46. Pottery jug. Thick walled, pinkish. With a clover-shaped lip and reddish paint. H: 19.3, DM: 10, DB: $7 \mathrm{~cm}$ (Pl. 5, No. 3).

47. Pottery beaker. Brownish-grey, with worn black paint. H: 8.6, DM: 7.8, DB: $4 \mathrm{~cm}(\mathrm{VI} / 8)^{29}(P l$. 4, No. 11).

48. Pottery urn. Brownish-grey, with a smooth red spot. Its surface is smoothed in horizontal lines. H: 14.4, DM: 10, DB: $6 \mathrm{~cm}(P l .2$, No. 15).

49. Pottery flagon with handle. Grey, rough, coarse. Scratched inscription on its neck. H: 13.6, DM: 7.8, DB: $5.7 \mathrm{~cm}$ (Pl. 5, No. 1).

In spite of the record made at the time of excavation, we cannot accept that 49 vessels were deposited in a single grave. Their original association with this Roman tomb is conclusively disproved by the inclusion in the find of the stove tile (No. 39) from the Turkish period (16-17th centuries A.D.). In our view this might have been a disturbed grave where articles which were found in the graves demolished in the vicinity were collected together.

Grave $\mathbf{R} / 1^{30}(P l$. 50). This is a child's grave, to the east of the south-eastern corner of burialchamber III. L: 72, W: 51, H: $40 \mathrm{~cm}$. Its ground plan was irregular. According to the description by Gy. Gosztonyi its longer wall joins the northern wall in an apse after a straight portion of $46 \mathrm{~cm}$. Its roof is made of three pairs of keyed bricks: $\Lambda$. D: $209 \mathrm{~cm}$. On the bricks lineal decoration is visible. Gy. Gosztonyi's description does not mention either a skeleton or associated articles. ${ }^{31}$

In burial-chamber III the burial rite followed in the cemetery was obviously used: the neighbouring graves surrounded the burial-chamber.

A list follows of some additional articles which are kept together with the material found in these graves. These articles could not be identified with specific graves.

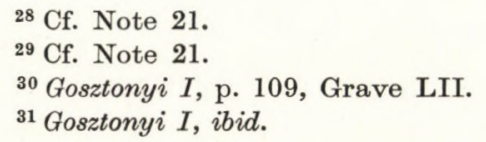

1. Beads made of glass paste. 85 items of flat, round shape, and of greyish-blue colour ( $P l$. 1, No. 3).

2. Bronze ring. Its inner edge is rounded. Diam: 3.7, W: 0.7 , Th: $0.3 \mathrm{~cm}$ (Pl. 1, No. 4).

3. Glass beaker. Yellowish-white, thin walled. With a narrow foot-ring. Complete. H: 7.4, DM: 8.6, DB: $3.8 \mathrm{~cm}$ (Pl. 1, No. 6).

4. Glass beaker. Greenish-white, thin walled. Engraved with horizontal lines below the rim. Its lip is broken. H: 6.4, DM: $8.4, \mathrm{DB}: 4.8 \mathrm{~cm}$ (Pl. 1, No. 7).

5. Coins:
(a) Tacitus
(b) Julian
Ant.
(C-XII. 1) $)^{31^{a}}$
(c) Valentinian
AE 3
(C-XII. 2)
(C-XII. 3)

\section{BURIAL-CHAMBER IV (Pl. 50)}

This was excavated in 1913 west of burialchamber I, and has painted walls. ${ }^{32}$ In front of its northern wall there is a sarcophagus and traces of another tomb can be seen on the southern side (Graves IV/1-2). W-E orientation.

Bronze key, with ring (before the chamber). L: $8.3, \mathrm{~W}: 2.8 \mathrm{~cm}$ ( $P l$. 1, No. 2).

Above burial-chamber IV three graves were found:

Graves R/20-21 ( $P l$. 50). Their longer walls were made of stone, the shorter ones of brick. Two were built closely side by side (double tomb?). W-E orientation.

Grave $\mathbf{R} / 22(P l .50)$. West of the earlier ones, its side walls were made of brick. Its cover was missing. 33

\section{BURIAL-CHAMBER V $(P l .50)$}

This was found in 1913 east of burial-chamber I with painted walls. ${ }^{34}$

\footnotetext{
31 $a$ The coins found on the territory of the whole town are classified in tables, of which this work uses tables C-XII, XIII and XVI.

32 O. Szőnyi, Dunántríl, 3 July and 25 December, 1913; Report of I. Möller: NCM Archives, 572/1913. On 28 July, 1913; Gosztonyi I, p. 117. Tomb chamber 5, further literature; Gosztonyi IV, p. 26, drawing 11, 1/a-c.

${ }^{33}$ O. Szőnyi, Dunántúl, 25 December 1913. About the shape of the grave Szőnyi writes the following: "The inside of the sarcophagus is not of regular oblong shape but the side walls corresponded to the curves of the human body."

${ }^{34}$ Gosztonyi $I$, p. 117, further literature; Gosztonyi IV, p. 30 , drawing 6 , g.
} 


\section{BURIAL-CHAMBER VI $(P l .50)$}

This was found in 1922 east of the obelisk adjoining the Bishop's Palace in the area in front of the cathedral. ${ }^{35}$ There are two graves in it made of stone and brick, respectively, and their roof is $\wedge$ shaped (Graves VI/1-2). N-S orientation.

Grave R/23-24 (Pl. 50). This was found in 1922, $1.50 \mathrm{~m}$ deep below the pavement of the western tower of the cathedral. ${ }^{36}$ It is a double grave, its walls were built of stone, covered inside with mortar. According to the description of O. Szönyi there were four cannonballs in one of the graves, three made of iron and one of lead. Two stone cannonballs were built into the southern wall of the other grave. On this basis he dates the graves after the 17th century A.D. In another paper ${ }^{37}$ O. Szőnyi mentions an apsed chamber whose wall was painted blue and red. According to his description one grave was found in this burial-chamber. In our view, based on a later description of O. Szőnyi ${ }^{38}$ and on a drawing by I. Möller in 1922, this may be a double tomb with painted walls which was subsequently rebuilt. But originally both were late Roman graves. In and around the graves human bones were lying in disorder with seven skulls among them.

\section{CELLA TRICHORA $(P l .50)$}

This was excavated in 1922 by O. Szőnyi and I. Möller. ${ }^{39}$ There are two graves below the ground in its narthex (Graves CT 1-2). W-E orientation.

Grave R/25 (Pl. 50). This was found in 1954 on the eastern side of the central apse of the cella trichora between the first buttress and the foundations of the cathedral. D, measured from the ground level of the cathedral: $200 \mathrm{~cm}$. Its northern wall is $20 \mathrm{~cm}$, the southern one is $18 \mathrm{~cm}$ thick and both may have been curved. Internal W: $45 \mathrm{~cm}$, measurable L: $100 \mathrm{~cm}, \mathrm{D}: 30 \mathrm{~cm}$. Its walls were built of brick and stone, and its bottom is covered with sand. The walls slant slightly upwards,,$^{40}$ and may

35 O. Szőnyi, Dunántúl, 19 November 1922; Gosztonyi $I$, p. 117 for further literature; Gosztonyi $I V .28$. drawing $11,2 / \mathrm{a}-\mathrm{b}$.

${ }^{36}$ O. Szőnyi, Dunántúl, 19 November, 1922.

${ }^{37}$ O. Szőnyi, Dunántúl, 2 July 1922.

${ }^{38}$ O. Szőnyi, Dunántúl, 19 November 1922.

${ }^{39}$ Entry DACL XIII. Pannonie, p. 1060; Szönyi II, p. 172 and supra; Gosztonyi $I$, p. 97 for further literature; Gosztonyi $I V$, p. 14 and supra for further literature; Fülep III, p. 152 for further literature. have been barrel vaulted. According to J. Dombay ${ }^{41}$ it is questionable whether the western end of the grave was joined to the cella. In the eastern end of the grave the shin-bones have been disturbed. W-E orientation. There were no associated articles.

Grave R/26 (Pl. 50). This was found $45 \mathrm{~cm}$ below the former one. The skeleton is mutilated, of W-E alignment, head to the west. Dombay inferred that there may have been some relationship between the lower skeleton and the burial structure (cella trichora). ${ }^{42}$

Between 26 and 29 November 1954, P. Lakatos conducted rescue excavations occasioned by the waterproofing of the cella trichora. ${ }^{43} \mathrm{He}$ unearthed three graves:

Graves R/27-29 (Pl. 50). The walls of the graves were built of brick and stone, and the majority of the bricks are of medieval character, but there are some Roman bricks as well. He mentions elongated burial structures resembling niches. Orientation: $\mathrm{W}-\mathrm{E}$, head to the west. There was one skeleton in each of the three graves. There were no associated articles. D: $50 \mathrm{~cm}$ below the present stone flooring.

Grave $\mathbf{R} / \mathbf{3 0}(\mathrm{Pl} .50)$. In front of the façade of the Bishop's Palace. W-E orientation. No other record is available. ${ }^{44}$

Grave R/31 ( $P l .50)$. This was found north of the above grave in the trench for I. Möller's sewer. ${ }^{45}$ $\mathrm{L}$ : (incomplete) $120 \mathrm{~cm}, \mathrm{~W}: 35 \mathrm{~cm}$ at the western end, $45 \mathrm{~cm}$ in the middle. Its eastern end is missing. Th. of walls: $25-30 \mathrm{~cm}$. Inferring from its shape, the two ends were narrower. I. Möller has reconstructed the complete form of the grave, according to which the inner L. was 170 , width at the eastern end was $30 \mathrm{~cm}$.

\section{BURIAL-CHAMBER VII $(P l .50)$}

We excavated this in 1958 to the west of the cella trichora. ${ }^{46}$ No grave was found in it. Originally

${ }^{40} \mathrm{Cf}$. with the Graves CT/1-2 found in the narthex of the cella trichora: Fülep $V$.

41 From the bequest of J. Dombay without signature and date: HNM Reference Archives, IV. 78/1965.

42 Ibid.

43 Report of P. Lakatos: 26 November 1954. JPM-Reference Archives, 19.

44 Szönyi $I I$, supplement No. 1.

45 Cf. Note 40.

46 Fülep $I$, p. 23 and supra. 
it may have contained one or two graves of $\mathrm{W}-\mathrm{E}$ orientation.

An additional grave was found below the narthex of the burial-chamber:

Grave $\mathbf{R} / 32(P l$. 50). This was a brick grave, its sides built of rimmed and plain bricks and its bottom also lined with bricks $(48 \times 38 \times 5 \mathrm{~cm}) .^{47}$ Its top was disturbed when the wall of the narthex was built so that the covering $\wedge$ bricks had fallen into the grave. L: 125 , D. of grave: $50 \mathrm{~cm}$. E-W orientation, head to the east. L. of skeleton: $110 \mathrm{~cm}$; the arms of the extended skeleton of a child were lying beside the body, parallel to it. There were no associated articles.

The orientation of the grave differs by 180 degrees from the general alignment in the early Christian cemetery of Pécs, as its head is to the east.

\section{CELLA SEPTICHORA $(P l .50)$}

This was found during the excavations conducted between 1938 and $1940 . .^{48}$ No grave was found in it.

Grave $\mathbf{R} / \mathbf{3 3}(P l .50)$. This was found at a depth of $200 \mathrm{~cm}$ in the infilled layer above the cella septichora. W-E orientation. It is an earth grave, and remains of an apparently female skeleton were found in it. ${ }^{49}$ According to the description, remains of jewels were also found beside the skeleton. The associated articles found and registered during the excavations of the cella septichora presumably belonged to this grave.

Associated articles:

1. Pottery spindle-whorl. It was of cream colour, and made from the wall of a vessel. Diam: 4.5, Th: $1.2 \mathrm{~cm}$. 7152/1941. (Pl. 10, No. 2).

2. Clay spindle-whorl. Light grey, conical, with a black spot. Diam: 2.2, Th: $1.3 \mathrm{~cm}$. 7152/1941 (Pl. 10, No. 1).

3. Mirror frame. Made of a flat strip of lead; round. Ornamented with embossed pelta and $\mathrm{V}$-shaped motifs among linear patterns. Diam: 5.3, W: 0.8, Th: $0.1 \mathrm{~cm}$. 7708/1941. (Pl. 10, No. 3).

47 Fülep I, p. 34, Pls 1 and 4; Pl. 6, 3-4.

48 Interview with Gy. Gosztonyi: Dunántúl, 18 February 1938; 20 February 1938; 23 February 1938; T. Horváth, Dunántúl, 22 March 1938; Gy. Gosztonyi, Dunántúl, 26 February 1939; Gy. Gosztonyi's reports: NCM Archives, 556/1939, 826/1939 and 406/1968. Excavation diary of Gy. Török: HNM Reference Archives I. 100/1968; Reports of A. Radnóti: HNM Reference Archives 77. P. II and 86. P. II; Gosztonyi I, p. 97 and supra for further literature; Gosztonyi, Arch. Ert. (1940) p. 56 and supra for further literature; Gosztonyi $I V, \mathrm{p}$ 10 and supra, drawing 1; Fülep III, p. 156.
In 1938 T. Horváth found two graves south of the cella septichora. ${ }^{50}$

Grave R/34 (Pl. 50). At a depth of $380 \mathrm{~cm}$. L: $90, \mathrm{~W}: 38$, D. of grave: $21 \mathrm{~cm}$. Its top was made of bricks $\wedge$. W-E orientation, head to the west. It contained a child's skeleton, in good condition, with both hands on the pelvis. There were bracelets on the lower left arm.

Associated articles:

1. Iron bracelet. Six fragments. Cylindrical cross section, made of wrought iron. Th: $0.3 \mathrm{~cm} .7157-2$ (Pl. 10, No. 6).

2. Bronze bracelet. It was stuck to the iron bracelet by rust. Made of bronze wire of $\mathrm{D}$ cross section, open. The two ends are stylized snake heads ornamented with a double dotted circle. Complete. Diam: 3.8, Th: $0.3 \mathrm{~cm}$. 7157-2 (Pl. 10, No. 7).

3. Fragments of a bone bracelet. Seven pieces. Yellowish-white, with dotted circle ornamentation. W: 0.6, Th: 0.2 cm. 7157-2a (Pl. 10, Nos 4-5).

Grave R/35 $(P l .50) .40 \mathrm{~cm}$ to the north of the former one. Their western end formed a single line. $405 \mathrm{~cm}$ below the surface. It had a masonry stone wall and its roof was made of big keyed bricks $\Lambda$; the roof was missing at the western end. L: 164, $\mathrm{W}$ : at the west 32 , at the east $45 \mathrm{~cm}$. W-E orientation. It contained an extended supine male skeleton, without associated articles.

In October 1940 Gy. Török found two burialchambers and four graves in the UpperPromenade..$^{51}$

\section{BURIAL-CHAMBER VIII (Török’s Chamber “A”)}

contained two graves: (Graves VIII/1-2), of which Grave VIII/2 was rebuilt, and shortened. W-E orientation $(P l .50)$.

\section{BURIAL-CHAMBER IX (Török's Chamber “B”),}

a grave was later built against its outer wall (Grave $\mathbf{I X / 1 ) . ~ W - E ~ o r i e n t a t i o n ~ ( P l . ~ 5 0 ) . ~}$

\footnotetext{
${ }^{49}$ Dunántúl, 20 February 1938.

${ }^{50}$ Excavation diary of T. Horváth: HNM Reference Archives X. 172/1968; T. Horváth, Dunántúl, 22 March 1938; Gosztonyi I, p. 115, Graves CXLI-CXLII.

51 Excavation diary of Gy. Török: HNM Reference Archives V. 113/1968; Excavation diary and report of A. Radnóti: HNM Reference Archives V. 109/1968; Török II, p. 207 and supra; Gosztonyi IV, p. 29, drawing 11, 4-5 and Pl. 4, No. 3.
} 
Grave R/36 (Török's Grave "C") ( $P l .50)$ ). Built of stone; its roof was made of brick $\wedge, L$ : 210 , $W: 110 \mathrm{~cm}$. The eastern end of its top was demolished. W-E orientation. Inside an adult skeleton was found without associated articles.

Grave R/37 (Török's Grave “D”) ( $P l .50)$. Built of stone; its roof was made of brick $\Lambda$, the middle of which is disturbed. L: 150, W: $80 \mathrm{~cm}$. It deviates from western orientation by 12 degrees. Inside there is a disturbed child's skeleton.

Associated article:

1. Coin. Claudius I. PB (lost)

Grave R/38 (Török's Grave “E”) ( $P l .50)$. Built of stone. Its western and southern end as well as its top were entirely missing. W-E orientation. Deviation from western orientation again by 12 degrees. Measurable W: $90 \mathrm{~cm}$. No skeleton or articles were found inside.

Grave R/39 (Török's Grave “F”) ( $P l$. 50). Made of stone, its vault (?) was destroyed. Only the western end was excavated. $W: 125 \mathrm{~cm}$, orientation: the same as that of Graves R/37-38. Gy. Török did not mention either a skeleton or associated articles.

In 1943 from the excavations of Gy. Török came to light:

Bronze bracelets. Two items.

a) D-shaped cross section, open, with stylized snake heads. Diam: 7, W: 0.5, Th: $0.4 \mathrm{~cm}$. 7195.4 (Pl. 10, No. 9).

b) Cylindrical cross section, open, with stylized snake heads. Diam: 6.1, Th: $0.1 \mathrm{~cm} 7195.5$ (Pl. 10, No. 8).
Grave $\mathbf{R} / \mathbf{4 0}(\mathrm{Pl}$. 50). This was found in 1964 to the west of burial-chamber II with painted walls, at a depth of $460 \mathrm{~cm}$. Its complete excavation was not possible. Measurable length: $120 \mathrm{~cm}$. Its wall was built of stone, its top $\wedge$ was of brick. $E-W$ orientation, head to the east. There was an extended supine skeleton of an adult in the grave, with the arms at the sides. In the excavated portion of the grave no articles were found. This grave also deviated by 180 degrees from the W-E orientation observed in the early Christian cemetery of Pécs; the head was found in the eastern end of the grave.

In 1970 Mária G. Sándor conducted rescue excavations at the north-western corner of the undercroft of the cathedral of Pécs. ${ }^{52} 40 \mathrm{~cm}$ below the floor of the undercroft a disturbed skeleton was found in an earth grave, and the legs were found at the eastern end of the grave (Grave $\mathbf{A} / \mathbf{I}$ ) (Pl. 50).

At a depth of $100 \mathrm{~cm}$ another supine extended skeleton was found in an earth grave. W-E orientation, head to the west. There were no associated articles (Grave A/II) $(P l$. 50).

At a depth of $90 \mathrm{~cm}$ a badly disturbed skeleton was found without articles. Orientation: the same as above (Grave A/III) ( $P l .50)$.

\section{BURIAL-CHAMBER X $(P l .50)$}

In 1927 a destroyed burial-chamber with painted walls was found in front of 6, Káptalan Street. We could identify the mosaic floor found here in 1841 with this burial-chamber. ${ }^{53}$
52 Haas, p. 9 mentions that many graves were found below the cathedral when the heating was installed; here I would like to thank G. Mária Sándor for having placed at my disposal the documentation of her excavation.
${ }^{5}$ Fülep V; Á. Kiss: Roman Mosaics in Hungary, Fontes Arch. Hung. (1973) p. 62. 


\begin{tabular}{|c|c|c|c|c|c|c|c|c|}
\hline No. & & & Av & Rv & RIC & A.D. & Grave & $\begin{array}{l}\text { INV No. } \\
\text { (PECS) }\end{array}$ \\
\hline 1. & $\begin{array}{l}\text { IMPCMCL TACITVS AVG } \\
\text { SALVS AVG }\end{array}$ & ANT & & $?$ & $\bar{?}$ & ? & - & $\mathrm{CT}$ \\
\hline 2. & $\begin{array}{l}\text { DN IVLIANVS NOB CAES } \\
\text { FEL TEMP-REPARATIO }\end{array}$ & AE3 & (P) & $\overline{\mathrm{ASIS}}$ & $\begin{array}{l}\text { LRBC } \\
1229\end{array}$ & $\begin{array}{l}\text { Siscia } \\
355-361\end{array}$ & $\longrightarrow$ & $\mathrm{CT}$ \\
\hline 3. & $\begin{array}{l}\text { DN VALENTINI-ANVS PF AVG } \\
\text { SECVRITAS REI PVBLICAE }\end{array}$ & EE3 & & $\frac{R}{A S I S C}$ & $147 / 15 / a$ & $\begin{array}{l}\text { Siscia } \\
367\end{array}$ & $\longrightarrow$ & $\mathrm{CT}$ \\
\hline 4. & $\begin{array}{l}\text { CONSTANTINVS IVN NOB C } \\
\text { GLORI-A EXERC-ITVS } \\
\end{array}$ & PB & & $\frac{2 \text { lab. }}{A S I S}$ & $\begin{array}{l}\text { LRBC } \\
743\end{array}$ & $\begin{array}{l}\text { Siscia } \\
330^{-}-335 \\
\end{array}$ & $\begin{array}{l}\text { IX. } \\
\text { chamber }\end{array}$ & 7715 \\
\hline 5. & $\begin{array}{l}\text { FL IVL CONSTANTIVS NOB C } \\
\text { GLOR-IA EXERC-ITVS }\end{array}$ & $\mathrm{PB}$ & & $\frac{2 \text { lab. }}{\text { SMTSA }}$ & 842 & $\begin{array}{l}\text { Thessalonica } \\
330-335\end{array}$ & $\begin{array}{l}\text { IX. } \\
\text { chamber }\end{array}$ & 7716 \\
\hline 6. & VRBS ROMA & PB & & $\overline{\mathrm{ONSA}}$ & 1008 & $\begin{array}{l}\text { Constantinopol } \\
330-335\end{array}$ & $\begin{array}{c}\text { IX. } \\
\text { chamber }\end{array}$ & 7717 \\
\hline 7. & $\begin{array}{l}\text { IMP.CAES·L·AVREL-VERVS } \cdot \text { AVG } \\
\text { TRP TR. P II tip. }\end{array}$ & GB & & - & Type 250 & Roma & $\mathrm{R} / 41$ & $805 / 1$ \\
\hline 8. & $\begin{array}{l}\text { CONSTANTINVS IVN NOB C } \\
\text { CAESARVM NOSTRORVM VOT/XX }\end{array}$ & $\mathrm{PB}$ & & $A \overline{S I S, *-}$ & $445 / 176$ & $\begin{array}{l}\text { Siscia } \\
321-324\end{array}$ & $\mathrm{R} / 41$ & $805 / 1$ \\
\hline 9. & \begin{tabular}{|l|l|} 
CALLIENVS AVG \\
VBERITAS AVG \\
\end{tabular} & ANT & & $E$ & $183 / 585 \mathrm{k}$ & $\begin{array}{l}\text { Siscia } \\
253-268 ?\end{array}$ & $\mathrm{R} / 42$ & 6110 \\
\hline 10. & $\begin{array}{l}\text { GALLIENVS AVG } \\
\text { IOVE: CONS AVG }\end{array}$ & ANT & & $\overline{S^{*}}$ & $149 / 207 . \mathrm{k}$ & $\begin{array}{l}\text { Roma } \\
253-268 ?\end{array}$ & $R / 42$ & 6113 \\
\hline 11. & $\begin{array}{l}\text { GALLIENVS AVG } \\
\text { IOVI CONS AVG } \\
\end{array}$ & ANT & & $\rho$ & $149 / 207 \mathrm{k}$ & $\begin{array}{c}\text { Roma } \\
253-268 ?\end{array}$ & $\mathrm{R} / 43$ & 6111 \\
\hline 12. & $\begin{array}{l}\text { GALLIENVS AVG } \\
\text { APOLLINI CONS ·AVG. }\end{array}$ & ANT & & $\bar{Z}$ & $145 / 166$ & $\begin{array}{c}\text { Roma } \\
253-268 ? \\
\end{array}$ & $\mathrm{R} / 43$ & 6112 \\
\hline 13 & $\begin{array}{l}\text { CONSTANTINVS MAX AVG } \\
\text { GLORI-A EXER-CITVS }\end{array}$ & PB & & $\frac{? l a b}{\text { SMTSA }}$ & $\begin{array}{l}\text { LRBC } \\
840 ? 845 ?\end{array}$ & $\begin{array}{c}\text { Thessalonica } \\
330-337\end{array}$ & - & - \\
\hline 14. & $\begin{array}{l}\text { IMP PRO-BVS AVG } \\
\text { SOLI-INVIC-TO }\end{array}$ & ANT & (ே) & $\overline{\mathrm{R}} \epsilon$ & $39 / 202 . G$ & $\begin{array}{c}\text { Roma } \\
276-282 ?\end{array}$ & $\mathrm{R} / 147$ & 6104 \\
\hline 15. & $\begin{array}{l}\text { IMP C M AVR PROBVS P AVG } \\
\text { SOLI INVICTO }\end{array}$ & ANT & & $\overline{K A \cdot A}$ & $112 / 864 . \mathrm{A}$ & $\begin{array}{l}\text { Serdica? } \\
276-282 ?\end{array}$ & $\mathrm{R} / 147$ & 6105 \\
\hline 16. & $\begin{array}{l}\text { IMP MAVR PROBVS PF AVG } \\
\text { CONCORDIA MILITVM }\end{array}$ & ANT & & $\overline{\mathrm{PXXT}}$ & $52 / 331 . G$ & $\begin{array}{l}\text { Ticinum } \\
276-282 ?\end{array}$ & $R / 148$ & 6106 \\
\hline 17. & $\begin{array}{l}\text { IMP CONSTANTINVS PF AVG } \\
\text { FIDES MILITVM AVGG ET CAES NN }\end{array}$ & AE2 & & $\overline{\mathrm{AQS}}$ & $\operatorname{con}^{2} 46$ & $\begin{array}{c}\text { Aquileia } \\
?\end{array}$ & $\mathrm{R} / 149$ & 6149 \\
\hline 18.5 & $\begin{array}{l}\text { IMP CONSTANTINVS P.F. } \\
\text { SACRA MONETA AVGG ET CAESS NOS }\end{array}$ & \begin{tabular}{|l|l|}
$E 2$ \\
$S T R$
\end{tabular} & & $\frac{*}{S Q}$ & $\operatorname{Coh}^{2} 264$ & $\begin{array}{c}\text { Aquileia } \\
?\end{array}$ & $\mathrm{R} / 149$ & 6150 \\
\hline 19. & \begin{tabular}{|l|} 
IMP LIC LICINIVS AVG \\
SAECVLI F-ELICITAS /AVG
\end{tabular} & FOLL & & $\frac{P R}{D S}$ & $315 / 160$ & $\begin{array}{c}\text { Roma } \\
318-319\end{array}$ & $R / 150$ & 5886 \\
\hline 20. & $\begin{array}{l}\text { DN CONSTAN-TIVS PF AVG } \\
\text { HOC SIG-NO VICTOR ERIS }\end{array}$ & E 2 & & $\frac{A}{A S I S *}$ & $\begin{array}{l}\text { LRBC } \\
1365\end{array}$ & $\begin{array}{c}\text { Siscia } \\
350\end{array}$ & $R / 150$ & 5887 \\
\hline 21. & $\begin{array}{l}\text { IMP PROBVS PF AVG } \\
\text { PAX AVGVSTI }\end{array}$ & ANT & & $\overline{\text { XXIV }}$ & $93 / 713 / c$ & $\begin{array}{l}\text { Siscia(?) } \\
276-282\end{array}$ & $\mathrm{R} / 178$ & 6131 \\
\hline 22. & $\begin{array}{l}\text { PROBV-S PF AVG } \\
\text { VICTOR-IA GER M }\end{array}$ & ANT & & $\overline{R \cup A}$ & $41 / 233$ & $\begin{array}{c}\text { Roma } \\
276-282 ?\end{array}$ & $\mathrm{R} / 170$ & $797 / 1$ \\
\hline 23 & $\begin{array}{l}\text { IMP PRO-BVS AVG } \\
\text { FIDES MILITVM }\end{array}$ & ANT & & $\overline{X X I P}$ & $91 / 694$ & $\begin{array}{c}\text { Siscia } \\
278-282 ?\end{array}$ & $R / 170$ & $797 / 1$ \\
\hline 24. & $\begin{array}{l}\text { IMP CM CARINVS PF AVG } \\
\text { VICTOR-IA GERMANICA }\end{array}$ & ANT & & - & $178 / 319(E)$ & $\begin{array}{l}\text { Cyzicus } \\
283-285 ?\end{array}$ & $\mathrm{R} / 170$ & $797 / 1$ \\
\hline 25. & \begin{tabular}{|l|} 
IMP PROBVS PF AVG \\
CONCORDIA MILIT \\
\end{tabular} & ANT & & $\overline{\mathrm{XXIP}}$ & $89 / 666 / A$ & $\begin{array}{l}\text { Siscia? } \\
276-282\end{array}$ & $\mathrm{R} / 178$ & 6130 \\
\hline 26.5 & $\begin{array}{l}\text { IMP DIOCLETIANVS PF AVG } \\
\text { SACRA MONETAVGG ET CAESS NOS }\end{array}$ & MB & & $\frac{B}{* S I S}$ & $469 / 131 / \mathrm{A}$ & $\begin{array}{c}\text { Siscia } \\
300\end{array}$ & $\mathrm{R} / 181$ & 6148 \\
\hline 27. $\mathrm{s}$ & \begin{tabular}{|l|} 
IMP C MAXIMIANVS PF AVG \\
SACRA MONET AVGG ET CAESS NOSTR
\end{tabular} & GB & & $\overline{\mathrm{P} . \mathrm{T}}$ & $286 / 47 / \mathrm{b}$ & $\begin{array}{l}\text { Ticinum } \\
304-305\end{array}$ & $R / 181$ & - \\
\hline 28. & \begin{tabular}{|l|} 
IMP LIC LICINIVS PF AVG \\
IOVI CON - SERVATOR
\end{tabular} & $M B$ & & $\overline{\text { ASIS }}$ & $426 / 22$ & $\begin{array}{l}\text { Siscia } \\
316 ?\end{array}$ & $R / 181$ & - \\
\hline
\end{tabular}




\begin{tabular}{|c|c|c|c|c|c|c|c|c|}
\hline No. & & & Av & $R v$ & RIC & A.D. & Grave & $\begin{array}{l}\text { INV. No } \\
\text { (PECS) }\end{array}$ \\
\hline 29. & $\begin{array}{l}\text { IMP CONSTANTINVS AVG } \\
\text { VICTORIAE LAETAE PRINCPERP/2 }\end{array}$ & $P B$ & $x^{\prime \prime \prime 2}=$ & $\frac{\text { VOTIPR }}{\text { IASIS* }}$ & $431 / 48$ & $\begin{array}{c}\text { Siscia } \\
318\end{array}$ & $\mathrm{R} / 181$ & - \\
\hline 30. & $\begin{array}{l}\text { CONSTAN - TINVS AVG } \\
\text { PROVIDEN - TIAE AVGG } 121\end{array}$ & PB & & $\overline{\mathrm{ASIS}}$ & $\begin{array}{c}\text { LRBC } \\
737\end{array}$ & $\begin{array}{l}\text { Siscia } \\
324-330\end{array}$ & $\mathrm{R} / 177$ & $\left.\begin{array}{l}6126 \\
6128\end{array}\right\}$ \\
\hline 31. & $\begin{array}{l}\text { CONSTAN-TINVS AVG } \\
\text { PROVIDEN - TIAE AVGG } 121\end{array}$ & PB & & $\overline{\text { SMTSA }}$ & 811 & $\begin{array}{c}\text { Thessal onica } \\
324-330\end{array}$ & $\mathrm{R} / 181$ & - \\
\hline 32. & \begin{tabular}{l|l} 
CONSTAN-TINVS AVG \\
PROVIDEN-TIAE AVGG
\end{tabular} & PB & & $\overline{\text { SMNA }}$ & 1107 & $\begin{array}{c}\text { Nicomedia } \\
324-330\end{array}$ & $\mathrm{R} / 177$ & 6127 \\
\hline 33. & $\begin{array}{l}\text { CONSTAN -TINVS AVG } \\
\text { PROVIDEN *TIAE AVGG } \\
\end{array}$ & PB & & $\overline{\text { SMNA }}$ & 1110 & $\begin{array}{l}\text { Nicomedia } \\
324-330\end{array}$ & $\mathrm{R} / 177$ & 6129 \\
\hline 34. & $\begin{array}{l}\text { CONSTAN-TINVS AVG } \\
\text { PROVIDEN-TIAE AVGG } 121\end{array}$ & PB & & $\overline{\text { SMKA }}$ & 1158 & $\begin{array}{l}\text { Cyzicus } \\
324-330\end{array}$ & $R / 181$ & - \\
\hline 35. & $\begin{array}{l}\text { DN VALENTINI-ANVS PF AVG } \\
\text { GLORIA RO-MANORVM }\end{array}$ & AE 3 & & $\frac{R}{B S I S C}$ & $147 / 14 / a$ & $\begin{array}{c}\text { Siscia } \\
367\end{array}$ & $R / 183$ & 6109 \\
\hline 36. & $\begin{array}{l}\text { DN VALENTINI-ANVS PF AVG } \\
\text { GLORIA RO-MANORVM }\end{array}$ & AE 3 & & $\frac{M \quad F}{B S I S C}$ & $147 / 14 / \alpha$ & $\begin{array}{c}\text { Siscia } \\
369\end{array}$ & $\mathrm{R} / 183$ & 6109 \\
\hline 37. & $\begin{array}{l}\text { DN VALENTINI-ANVS PF AVG } \\
\text { GLORIA RO-MANORVM }\end{array}$ & E 3 & & $\frac{*}{\operatorname{CONSA}}$ & $219 / 41 / a$ & $\begin{array}{c}\text { Constantinopolis } \\
367-375\end{array}$ & $R / 183$ & 6109 \\
\hline 38. & $\begin{array}{l}\text { DN VALENTINI-ANVS PF AVG } \\
\text { SECVRITAS REI PVBLICAE }\end{array}$ & ES3 & & $\frac{\mathrm{R}}{\mathrm{\triangle SISC}}$ & $147 / 15 / a$ & \begin{tabular}{|c|} 
Siscia \\
368 \\
\end{tabular} & $R / 183$ & 6109 \\
\hline 39. & $\begin{array}{l}\text { DN VALENTINI ANVS PF AVG } \\
\text { SECVRITAS REI PVBLICAE }\end{array}$ & E 3 & & $\frac{P}{\Gamma S I S C}$ & $147 / 15 / a$ & $\begin{array}{c}\text { Siscia } \\
370\end{array}$ & $R / 183$ & 6109 \\
\hline 40. & $\begin{array}{l}\text { DN VALEN-S PF AVG } \\
\text { GLORIA RO-MANORVM }\end{array}$ & EE 3 & & $\mathrm{BSISC}$ & $146 / 5 / b$ & $\begin{array}{c}\text { Siscia } \\
366\end{array}$ & $R / 183$ & 6137 \\
\hline 41. & $\begin{array}{l}\text { DN VALEN-S PF AVG } \\
\text { GLORIA RO-MANORVM }\end{array}$ & E 3 & & $\frac{\pi}{B S I S C}$ & $146 / 5 / b$ & $\begin{array}{c}\text { Siscia } \\
367\end{array}$ & $\mathrm{R} / 183$ & 6136 \\
\hline 42. & $\begin{array}{l}\text { DN VALEN-S PF AVG } \\
\text { SECVRITAS REI PVBLICAE }\end{array}$ & $A \in 3$ & & $\overline{\text { DASISC }}$ & $146 / 7 / b$ & $\begin{array}{r}\text { Siscia } \\
364 ?\end{array}$ & $\mathrm{R} / 183$ & 6139 \\
\hline 43. & $\begin{array}{l}\text { DN VALEN S PF AVG } \\
\text { SECVRITAS REI PVBLICAE }\end{array}$ & E 3 & & $\frac{*}{D A S I S C}$ & $146 / 7 / b$ & $\begin{array}{c}\text { Siscia } \\
367 \\
\end{array}$ & $R / 183$ & 6138 \\
\hline 44. & $\begin{array}{l}\text { IMP C PROBVS PF AVG } \\
\text { ABVNDANTIA AVG }\end{array}$ & ANT & & IIIII & $22 / 17$ & $\begin{array}{l}\text { Lugdunum } \\
276-282 ?\end{array}$ & $R / 184$ & 6107 \\
\hline 45. & $\begin{array}{l}\text { IMP PROBVS PF AVG } \\
\text { PAX AVGVSTI }\end{array}$ & ANT & - & $\frac{P}{x x \mid}$ & $93 / 713 / 9$ & $\begin{array}{l}\text { Siscia } \\
276-282 ?\end{array}$ & $\mathrm{R} / 184$ & 6108 \\
\hline 46. & $\begin{array}{l}\text { IMP DIOCLET IANVS PF AVG } \\
\text { SACRMONET AVGG ETCAESS NOSTR }\end{array}$ & $M B$ & & $\frac{\mathrm{*} V \mathrm{VI}}{\mathrm{AQP}}$ & $316 / 37 / a$ & $\begin{array}{c}\text { Aquileia } \\
303\end{array}$ & $\mathrm{R} / \mathrm{XI} \quad \mathrm{I}$ & 6125 \\
\hline 47. & $\begin{array}{l}\text { IMP.CAES NERVAE-TRAIANO AVG } \\
\text { GER } \cdot \text { DAC } \cdot P \cdot M \cdot T R \cdot P \cdot C O S \cdot V \cdot P \cdot P \cdot \\
S \cdot P \cdot Q \cdot R \cdot O P T I M O \text { PRINCIPI S.C }\end{array}$ & MB & & SC & $279 / 483$ & $\begin{array}{c}\text { Roma } \\
103-111\end{array}$ & $\mathrm{R} / 191$ & $\begin{array}{c}\text { HNM } \\
108 / 1912.15\end{array}$ \\
\hline 48. & $\begin{array}{l}\text { IMP. C. AVRELIANVS AVG. } \\
\text { CONCORDIA MILITVM. }\end{array}$ & ANT & & $\overline{P *-V \| *}$ & $271 / 59$ & $\begin{array}{c}\text { Roma } \\
?\end{array}$ & - & 1363 \\
\hline 49. & $\begin{array}{l}\text { IMP C.PROBVS AVG } \\
\text { CONCORD. MILIT. }\end{array}$ & ANT & & $\overline{A X X I-S X X I}$ & $73 / 532$ & $\begin{array}{c}\text { Ticinum } \\
?\end{array}$ & - & 1364 \\
\hline 50 & $\begin{array}{l}\text { IMP LICINIVS P.F AVG. } \\
\text { IOVI CONSERVATORI }\end{array}$ & ANT & & - & $\operatorname{coh}^{2} 73$ & - & - & 1365 \\
\hline 51. & $\begin{array}{l}\text { DN CONSTAN-TIVS PF AVG } \\
\text { FEL TEMP-REPARATIO }\end{array}$ & E3 & & $\overline{A \cdot S I S}$ & $\begin{array}{l}\text { LRBC } \\
1225\end{array}$ & $\begin{array}{l}\text { Siscia } \\
355-361\end{array}$ & $\mathrm{R} / 223$ & 4769 \\
\hline 52. & $\begin{array}{l}\text { DN VALEN-S PF AVG } \\
\text { GLORIA RO-MANORVM }\end{array}$ & EE3 & & $\frac{\mathrm{R}}{\mathrm{BSISC}}$ & $147 / 14 / 0$ & $\begin{array}{c}\text { Siscia } \\
367\end{array}$ & $R / 234-237$ & - \\
\hline 53. & $\begin{array}{l}\text { GALLIENVS AVG } \\
\text { APOLLINI CONS AVG }\end{array}$ & ANT & & & $145 / 166$ & Roma & $R / 250$ & 6120 \\
\hline 54. & $\begin{array}{l}\text { GALLIENVS AVG } \\
\text { FORTVNA REDVX }\end{array}$ & ANT & & $\mathrm{S}$ & $181 / 572 / \mathrm{A}$ & Siscia & $R / 250$ & 6121 \\
\hline 55. & $\begin{array}{l}\text { GALLIENVS AVG } \\
\text { FIDES EXERC VIII }\end{array}$ & ANT & & $P$ & $173 / 478 / K$ & Mediolanum & $R / 250$ & 6122 \\
\hline 56. & $\begin{array}{l}\text { DN VALEN-S PF AVG } \\
\text { SECVRITAS REI PVBLICAE }\end{array}$ & E 3 & $\therefore \ddot{\theta}$ & $\frac{*}{D A S I S C}$ & $146 / 7 / \mathrm{b}$ & $\begin{array}{l}\text { Siscia } \\
367\end{array}$ & - & - \\
\hline
\end{tabular}




\begin{tabular}{|c|c|c|c|c|c|c|c|c|}
\hline No. & & & Av & $R v$ & RIC & A.D. & Grave & $\begin{array}{l}\text { INV No. } \\
\text { (PECS) }\end{array}$ \\
\hline 57. & $\begin{array}{l}\text { DN CONSTAN-TINVS AVG } \\
\text { PROVDEN-TIAE AVGG }\end{array}$ & PB & & $\overline{\text { SMNA }}$ & $\begin{array}{c}\text { LRBC } \\
1110 \\
\end{array}$ & $\begin{array}{c}\text { Nicomedia } \\
324-330\end{array}$ & - & 6367 \\
\hline 58. & $\begin{array}{l}\text { DN IVLIAN-VS NOBC } \\
\text { FEL TEMP REPARATIO }\end{array}$ & PB & & $\overline{\mathrm{M}}$ & 1610 & $\begin{array}{r}\text { Sirmium } \\
355-361 \\
\end{array}$ & - & 6369 \\
\hline 59. & $\begin{array}{l}\text { CONSTANS NOB CAES } \\
\text { GLORI-A EXERC-ITVS } \\
\end{array}$ & PB & & $\frac{2 l a b}{\text { SMTSA }}$ & 843 & $\begin{array}{c}\text { Thessalonica } \\
330-335 \\
\end{array}$ & $R / 264$ & 3444 \\
\hline 60. & DN CONSTANTI-NVS PF AVGG & PB & & $\overline{\text { SMAL }}$ & 1469 & $\begin{array}{c}\text { Alexandria } \\
341-346\end{array}$ & $R / 273$ & $\begin{array}{c}\text { HNM } \\
/ 1337 / 1 . / E /\end{array}$ \\
\hline 61. & $\begin{array}{l}\text { DN CONSTA-NS PF AVG } \\
\text { FEL TEMP REPARATIO } \\
\end{array}$ & EE2 & & $\overline{\mathrm{ASIS}}$. & 1140 & $\begin{array}{c}\text { Siscia } \\
346-350 \\
\end{array}$ & $R / 269$ & $11337 / 10$ \\
\hline 62. & \begin{tabular}{|l|} 
N CONSTA-NS PF AVG \\
VOT IXX/MULT/XXX $\mid 1+1 ? /$ \\
\end{tabular} & PB & & $\overline{\text { SMNA }}$ & 1150 & $\begin{array}{l}\text { Nicomedia } \\
341-346\end{array}$ & $R / 273$ & $11337 / 3$ \\
\hline 63. & $\begin{array}{l}\text { D N CONSTA-NS PF AVG } \\
\text { VOT/XX/MVLT/XXX. } \\
\end{array}$ & PB & & $\overline{S M K B}$ & 1303 & $\begin{array}{l}\text { Cyzicus } \\
341-346\end{array}$ & $R / 273$ & $/ 1337 / 3$ \\
\hline 64. & $\begin{array}{l}\text { CONSTANS PF AVG } \\
\text { VICTORIAE DD AVGG Q NN } / 3 /\end{array}$ & PB & & $\bar{?}$ & Coh? $^{2} 176$ & - & $R / 273$ & $/ 1337 / 2$ \\
\hline 65. & $\begin{array}{l}\text { COSTANT-IVS PF AVG } \\
\text { VICTORIAE DD AVGG Q NN } \\
\end{array}$ & $\mathrm{PB}$ & & $\overline{\mathrm{AQP}}$ & $\begin{array}{c}\text { LRBC } \\
701 \\
\end{array}$ & $\begin{array}{l}\text { Aquileia } \\
341-346\end{array}$ & $\mathrm{R} / 273$ & $/ 1337 / 7$ \\
\hline 66. & $\begin{array}{l}\text { CONSTAN-TIVS AVG } \\
\text { GLORIA EXERCITVS } \\
\end{array}$ & PB & & $\frac{1 \text { lab. }}{\text { SMAN }}$ & 1379 & $\begin{array}{l}\text { Antiochia } \\
337-341\end{array}$ & $\mathrm{R} / 273$ & $/ 1337 / 5$ \\
\hline 67. & $\begin{array}{l}\text { DN CONSTAN-TIVS PF AVG } \\
\text { VOT/XX/MVLT/XXX. } \\
\end{array}$ & PB & & $\overline{\text { SMANA }}$ & 1398 & $\begin{array}{c}\text { Antiochia } \\
341-346\end{array}$ & $\mathrm{R} / 273$ & $/ 1337 / 8$ \\
\hline 68. & $\begin{array}{l}\text { FL IVL CONSTANTIVS NOB C } \\
\text { HOC SIG-NO VICTOR ERIS }\end{array}$ & $\mathbb{E} 2$ & A 少 & $\frac{A}{-A S I S}$ & 1173 & $\begin{array}{c}\text { Siscia } \\
350\end{array}$ & $\mathrm{R} / 273$ & $/ 1337 / 6$ \\
\hline 69. & $\begin{array}{l}\text { DN CONSTAN-TIVS PF AVG } \\
\text { VOT/XX/MVLT/XXX } \\
\end{array}$ & PB & & $\overline{\text { SMALB }}$ & 1474 & $\begin{array}{l}\text { Alexandria } \\
341-346\end{array}$ & $\mathrm{R} / 273$ & $/ 1337 / 8$ \\
\hline 70. & $\begin{array}{l}\text { DN CONSTANTIVS IVN NOB C } \\
\text { FEL TEMP REPARATIO } / 51 \\
\end{array}$ & AE2 & & $?$ & $\operatorname{coh}^{2} 5$ & 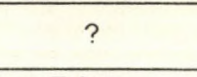 & $\mathrm{R} / 273$ & $/ 1337 / 9$ \\
\hline 71. & $\begin{array}{l}\text { DN CONSTAN-TIVS PF AVG } \\
\text { FEL.TEMP-REPARATIO } \\
\end{array}$ & E2 & & $\frac{A}{\text { TRP }}$ & $\begin{array}{l}\text { LRBC } \\
42\end{array}$ & $\begin{array}{c}\text { Trier } \\
346-350 \\
\end{array}$ & $\mathrm{R} / 273$ & $/ 1337 / 4$ \\
\hline 72. & $\begin{array}{l}\text { DN CONSTAN-TIVS PF AVG } \\
\text { FEL TEMP-REPARATIO }\end{array}$ & $E 2$ & & $\frac{\text { III }}{\text { BSIS }}$ & 1202 & $\begin{array}{c}\text { Siscia } \\
351-354 \\
\end{array}$ & $\mathrm{R} / 273$ & $/ 1337 / 4$ \\
\hline 73. & $\begin{array}{l}\text { DN CONSTAN-TIVS PF AVG } \\
\text { FEL TEMP-REPARATIO }\end{array}$ & $E 2$ & & $\frac{A}{A Q P}$ & 893 & $\begin{array}{l}\text { Aquileia } \\
346-350\end{array}$ & $R / 273$ & $/ 1337 / 4$ \\
\hline 74 & $\begin{array}{l}\text { DN CONSTAN-TIVS PF AVG } \\
\text { FEL TEMP-REPARATIO }\end{array}$ & $E 2$ & A $1 \%=$ & $\frac{A}{A Q S}$. & 893 & $\begin{array}{l}\text { Aquileia } \\
346-350\end{array}$ & $R / 273$ & $/ 1337 / 4$ \\
\hline 75. & $\begin{array}{l}\text { IMP LIC LICINIVS PF AVG } \\
\text { IOVI CONS-ERVATORI } \quad 121 \\
\end{array}$ & FOU & & $\frac{A}{S I S}$ & $423 / 8$ & $\begin{array}{r}\text { Siscia } \\
313-315 \\
\end{array}$ & $R / 275$ & $\left.\begin{array}{l}6140 \\
6141\end{array}\right\}$ \\
\hline 76. & $\begin{array}{l}\text { IMP LIC LICINIVS PF AVG } \\
\text { IOVI CONS-ERVATORI } 121\end{array}$ & FOLL & & $\frac{\mathrm{B}}{\mathrm{SIS}}$ & $423 / 8$ & $\begin{array}{r}\text { Siscia } \\
313-315 \\
\end{array}$ & $R / 275$ & 6142 \\
\hline 77. & $\begin{array}{l}\text { IMP CONSTANTINVS PF AVG } \\
\text { SOLI INVI-C-TO COMITI } 121 \\
\end{array}$ & PB & & $\overline{\mathrm{PT}}$ & $366 / 43$ & $\begin{array}{c}\text { Ticinum } \\
316 \\
\end{array}$ & $R / 275$ & $\left.\begin{array}{l}6154 \\
6155\end{array}\right\}$ \\
\hline 78. & $\begin{array}{|ll|}\text { CONSTAN-TINVS } & \text { AVG } \\
\text { DN CONSTANTINI } & \text { MAX AVG } \\
\end{array}$ & PB & & $\frac{\text { VOTXX }}{\text { ASISIM }}$ & $446 / 180$ & $\begin{array}{c}\text { Siscia } \\
321-324 \\
\end{array}$ & $\mathrm{R} / 275$ & 6156 \\
\hline
\end{tabular}




\begin{tabular}{|c|c|c|c|c|c|c|c|}
\hline No. & & & Rv & LRBC & A.D. & $\begin{array}{l}\text { INV. No. } \\
\text { (PECS) }\end{array}$ & Grave \\
\hline 1. & VRBS ROMA & PB & $\overline{\mathrm{R} *}$ & 563 & $\begin{array}{c}\text { Roma } \\
335-337\end{array}$ & 1640 & $\mathrm{R} / 212$ \\
\hline 2. & VRBS ROMA & PB & $\overline{\text { SMHA }}$ & 902 & $\begin{array}{r}\text { Heraclea } \\
330-335\end{array}$ & 1630 & $R / 212$ \\
\hline 3. & VRBS ROMA & PB & $\overline{\text { SMKA }}$ & 1231 & $\begin{array}{l}\text { Cyzicos } \\
330-335\end{array}$ & 1631 & $\mathrm{R} / 212$ \\
\hline 4. & $\begin{array}{l}\text { CONSTANTINVS MAX AVG } \\
\text { GLORIA EXERCITVS }\end{array}$ & PB & $?$ & \multicolumn{2}{|c|}{ not identifiable } & 1637 & $R / 212$ \\
\hline 5. & $\begin{array}{l}\text { CONSTANTINVS IVN NOB C } \\
\text { GLORI-A EXERC-ITVS } \\
\end{array}$ & PB & $\overline{\text { SMTSA }}$ & 846 & $\begin{array}{c}\text { Thessalonica } \\
330-337 \\
\end{array}$ & 1639 & $\mathrm{R} / 2: 2$ \\
\hline 6. & $\begin{array}{l}\text { CONSTANTINVS PF AVG } \\
\text { GLOR-IA EXER-CITVS } \\
\end{array}$ & PB & $\overline{\text { SMTSA }}$ & 854 & $\begin{array}{c}\text { Thessalonica } \\
337-341\end{array}$ & 1638 & $\mathrm{R} / 212$ \\
\hline 7. & $\begin{array}{l}\text { CONSTANTINVS IVN NOB C } \\
\text { GLOR-IA EXERC-ITVS }\end{array}$ & PB & $\overline{\text { SMNA }}$ & 1126 & $\begin{array}{c}\text { Nicomedia } \\
335-337\end{array}$ & 1634 & $\mathrm{R} / 212$ \\
\hline 8. & $\begin{array}{l}\text { CONSTAN-S PF AVG } \\
\text { GLOR-IA EXERC-ITVS }\end{array}$ & PB & $\overline{\mathrm{ASIS}}$ & 778 & $\begin{array}{l}\text { Siscia } \\
337-341 \\
\end{array}$ & 1633 & $\mathrm{R} / 212$ \\
\hline 9 & $\begin{array}{l}\text { FL IVL CONSTANTIVS NOB C } \\
\text { GLOR-IA EXERC-ITVS }\end{array}$ & PB & $\overline{R \notin P}$ & $?$ & $\begin{array}{c}\text { Roma } \\
335-337\end{array}$ & 1643 & $\mathrm{R} / 212$ \\
\hline 10. & $\begin{array}{l}\text { FL IVL CONSTANTIVS NOB C } \\
\text { GLOR-IA EXERC - ITVS }\end{array}$ & $\mathrm{PB}$ & $?$ & \multicolumn{2}{|c|}{ not identifiable } & 1659 & $\mathrm{R} / 212$ \\
\hline 11. & $\begin{array}{l}\text { FL IVL CONSTANTIVS NOB C } \\
\text { GLOR-IA EXERC-ITVS } \\
\end{array}$ & PB & $\overline{\mathrm{ASIS}}$ & 756 & \begin{tabular}{|c|} 
Siscia \\
$335 \cdot 337$ \\
\end{tabular} & 1636 & $\mathrm{R} / 212$ \\
\hline 12. & \begin{tabular}{|l|l|} 
CONSTANTI-VS PF AVG \\
VICTORIAEDDAVGGQNN $/ 3 /$ \\
\end{tabular} & PB & $\overline{\mathrm{ASIS}}$ & 790 & $\begin{array}{l}\text { Siscia } \\
341-346\end{array}$ & $\left.\begin{array}{l}1641 \\
1642 \\
1655\end{array}\right\}$ & $\begin{array}{l}R / 212 \\
R / 212 \\
R / 214\end{array}$ \\
\hline 13. & $\begin{array}{l}\text { DN CONSTAN -TIVS PF AVG } \\
\text { FEL TEMP-REPARATIO } \\
\end{array}$ & E 3 & $\frac{M}{\text { ASIRM }}$ & 1609 & $\begin{array}{l}\text { Sirmium } \\
335-361\end{array}$ & 1584 & $\mathrm{R} / 204$ \\
\hline 14 & $\begin{array}{l}\text { FL IVL CONSTANTIVS NOBC } \\
\text { GLOR-IA EXERC-ITVS. }\end{array}$ & PB & $\overline{\text { SMHA }}$ & 937 & $\begin{array}{r}\text { Heraclea } \\
335-337 \\
\end{array}$ & 1632 & $\mathrm{R} / 212$ \\
\hline 15. & $\begin{array}{l}\text { CONSTAN-TIVS AVG } \\
\text { GLOR-IA EXERC-ITVS }\end{array}$ & PB & $\overline{\text { SMHA }}$ & $94 \varepsilon$ & \begin{tabular}{|r|} 
Heraclea \\
$337-341$ \\
\end{tabular} & 1635 & $\mathrm{R} / 212$ \\
\hline 16. & $\begin{array}{l}\text { DN CONSTAN-TIVS PF AVG } \\
\text { GLOR -IA EXERC-ITVS }\end{array}$ & PB & $\overline{\text { CONSA }}$ & 1043 & $\begin{array}{c}\text { Constantinopol } \\
337-341 \\
\end{array}$ & 1656 & $R / 214$ \\
\hline 17. & $\begin{array}{l}\text { DN CONSTANTIVS NOB... } \\
\text { FEL TEMP..... }\end{array}$ & E3 & $?$ & \multicolumn{2}{|c|}{ not identifiable } & 1585 & $\mathrm{R} / 204$ \\
\hline 18. & $\begin{array}{l}\text { DN IOVIA-NVS PF AVG } \\
\text { VOT /V / MVLT/X }\end{array}$ & AE3 & $\overline{\mathrm{ASISC}}$ & 1267 & $\begin{array}{l}\text { Siscia } \\
363-364\end{array}$ & 1583 & $\mathrm{R} / 204$ \\
\hline 19. & $\begin{array}{l}\text { DN IOVIA-NVS PF AVG } \\
\text { VOT/VI MVLTIX }\end{array}$ & AE3 & $\bar{?}$ & \multicolumn{2}{|c|}{ not identifiable } & 1613 & $R / 207$ \\
\hline 20. & $\begin{array}{l}\text { DN VALENTINI-ANVS PF AVG } \\
\text { GLORIA RO-MANORVM }\end{array}$ & ES3 & $\overline{\mathrm{ASISC}}$ & $\begin{array}{l}\mathrm{RIC} \\
146.5 / \mathrm{a}\end{array}$ & $\begin{array}{l}\text { Siscia } \\
364-367\end{array}$ & 1601 & $R / 207$ \\
\hline 21. & $\begin{array}{l}\text { DN VALENTINI-ANVS PF AVG } \\
\text { GLORIA RO-MANORVM } \\
\end{array}$ & EE3 & $\frac{\vec{A}}{\Gamma \operatorname{SISC}}$ & 146. $5 / a$ & $\begin{array}{l}\text { Siscia } \\
364-367\end{array}$ & 1602 & $R / 207$ \\
\hline 22 & $\begin{array}{l}\text { DN VALENTINI-ANVS PF AVG } \\
\text { GLORIA RO-MANORVM } 121\end{array}$ & ES3 & $\frac{\mathrm{R}}{\mathrm{BSISC}}$ & 147. $14 / a$ & $\begin{array}{l}\text { Siscia } \\
367-375 \\
\end{array}$ & $\begin{array}{l}1607 \\
1608\end{array}$ & $\mathrm{R} / 207$ \\
\hline 23. & $\begin{array}{l}\text { DN VALENTINI-ANVS PF AVG } \\
\text { GLORIA RO-MANORVM }\end{array}$ & E3 & $\frac{S O D}{B S I S C}$ & 147. $14 / a$ & $\begin{array}{l}\text { Siscia } \\
367-375\end{array}$ & 1611 & $\mathrm{R} / 207$ \\
\hline 24. & $\begin{array}{l}\text { DN-VALENTINI-ANVS PF AVG } \\
\text { GLORIA RO-MANORVM }\end{array}$ & EE3 & $\frac{M P}{B S I S C}$ & 147. $14 / a$ & $\begin{array}{l}\text { Siscia } \\
367-375\end{array}$ & 1596 & $R / 207$ \\
\hline 25. & $\begin{array}{l}\text { DN VALENTINI-ANVS PF AVG } \\
\text { GLORIA RO-MANORVM } 131 \\
\end{array}$ & AE 3 & $?$ & \multicolumn{2}{|c|}{ not identifiable } & $\left.\begin{array}{l}1603 \\
1606 \\
1610\end{array}\right\}$ & $\mathrm{R} / 207$ \\
\hline 26. & $\begin{array}{l}\text { DN VALENTINI-ANVS PF AVG } \\
\text { SECVRITAS-REI PVBLICAE I2 }\end{array}$ & E3 & $\frac{*}{\Delta \mathrm{SISC}}$ & $146.7 / a$ & $\begin{array}{l}\text { Siscia } \\
364-367\end{array}$ & $\begin{array}{l}1600 \\
1609\end{array}$ & $\mathrm{R} / 207$ \\
\hline 27. & $\begin{array}{l}\text { DN VALENTINI-ANVS PF AVG } \\
\text { SECVRITAS-REI PVBLICAE / } / 2 \\
\end{array}$ & E3 & $\frac{D S}{\Delta S I S C}$ & 147. $15 / a$ & $\begin{array}{l}\text { Siscia } \\
367-375 \\
\end{array}$ & $\left.\begin{array}{l}1597 \\
1599\end{array}\right\}$ & $\mathrm{R} / 207$ \\
\hline 28 & $\begin{array}{l}\text { DN VALENTINI -ANVS PF AVG } \\
\text { SECVRITAS REI PVBLICAE /2/ }\end{array}$ & $A E 3$ & $\frac{M}{\Delta S I S C}$ & 147. $15 / a$ & $\begin{array}{l}\text { Siscia } \\
367-375\end{array}$ & $\left.\begin{array}{l}1598 \\
1605\end{array}\right\}$ & $\mathrm{R} / 207$ \\
\hline 29. & $\begin{array}{l}\text { DN VALENTINI ANVS PF AVG } \\
\text { SECVRITAS-REI PVBLICAE } \\
\end{array}$ & $A E 3$ & $\frac{0^{R} \dot{M}}{\Gamma \operatorname{SISC}}$ & 147. $15 / a$ & $\begin{array}{l}\text { Siscia } \\
367-375\end{array}$ & 1604 & $\mathrm{R} / 207$ \\
\hline 30. & $\begin{array}{l}\text { DN VALENTINI-ANVS PF AVG } \\
\text { SECVRITAS-REI PVBLICAE }\end{array}$ & E3 & $?$ & \multicolumn{2}{|c|}{ not identifiable } & 1614 & $\mathrm{R} / 207$ \\
\hline 31. & $\begin{array}{l}\text { DN GRATIANVS PF AVG } \\
\text { GLORIA RO -MANORVM }\end{array}$ & E 3 & $\frac{D}{* B S I S C}$ & 147. $14 / c$ & \begin{tabular}{|l|} 
Siscia \\
$367-375$ \\
\end{tabular} & 1612 & $R / 207$ \\
\hline
\end{tabular}




\begin{tabular}{|c|c|c|c|c|c|c|c|}
\hline No. & & & Av & $R v$ & RIC & A.D. & $\begin{array}{l}\text { INV } \\
\text { (PECS }\end{array}$ \\
\hline 1. & \begin{tabular}{|l|} 
CRISPVS NOB CAES \\
CAESARVM NOSTRORVM VOTV \\
\end{tabular} & PB & $i=\frac{1}{(C)}$ & $\overline{A Q T}$ & $402 / 68$ & $\begin{array}{l}\text { Aquileia } \\
320-321\end{array}$ & $\longrightarrow$ \\
\hline 2. & $\begin{array}{l}\text { DN FL CL IVLII-ANVS PF AVG } \\
\text { VOT/X-MVLT/XX }\end{array}$ & PB & i $\%=$ & $\overline{\text { ASIRM }}$ & $\begin{array}{l}\text { LRBC } \\
1619\end{array}$ & $\begin{array}{l}\text { Sirmium } \\
361-363\end{array}$ & 61.150 .1 \\
\hline 3. & $\begin{array}{l}\text { IMP TRAIANVS DECIVS AVG } \\
\text { P MS C-OL VIM }\end{array}$ & GB & $\% \%$ & $\overline{\text { ANXII }}$ & $\begin{array}{l}\text { PICK } \\
130\end{array}$ & $\begin{array}{c}\text { Viminacium } \\
?\end{array}$ & 58.10 .3 \\
\hline 4 & $\begin{array}{l}\text { FAVSTINA-AVGVSTAE } \\
\text { SALVTI AVGVSTAE } \\
\end{array}$ & AVR & - & - & $\begin{array}{c}\text { BMC } \\
405 / 152\end{array}$ & $\begin{array}{c}\text { Roma } \\
161-176\end{array}$ & 61. 22.1 \\
\hline 5. & $\begin{array}{l}\text { IMP.C.CLAVDIVS P.F.AVG } \\
\text { VICTORIA AVG }\end{array}$ & AVR & $x^{1}=\bar{P}=$ & - & $\operatorname{coh}^{2} 291$ & - & 61.21 .1 \\
\hline 6. & $\begin{array}{l}\text { FL-IVL-CONSTANTIVS PERP.AVG } \\
\text { GLORIA. REI. PVBLICAE }\end{array}$ & AVR & $\frac{E=7}{(-2)}$ & $\begin{array}{l}\text { VOT IXXXV } \\
\text { MVLT XXXX } \\
\text { SIRM }\end{array}$ & $\operatorname{Coh}^{2} 123$ & Sirmium & 61. 23.1 \\
\hline
\end{tabular}




\section{ISTVÁN (SCITOVSZKY) SQUARE (Suppl. 2)}

In 1888 a vaulted cellar was found in one of the houses on the square. According to Gy. Gosztonyi ${ }^{54}$ it was a burial vault.

\section{2, ISTVÁN SQUARE (Suppl. 2)}

On this site fragments of a mosaic floor were found. 55

\section{CORNER OF ISTVÁN SQUARE AND JANUS PANNONIUS (KARDOS KÁLMÁN) STREET (Suppl. 2)}

Grave $\mathbf{R} / \mathbf{4 1}$. In front of the Littke building, at the corner of Kardos Kálmán Street, found during drainage works in 1927 ; $\mathrm{D}: 310 \mathrm{~cm}^{.56}$

Associated articles:

1. Bones. 34 pieces.

2. Roman bricks. 12 items.

3. Fragments of a glass bottle. Yellowish-white, thin walled. Diota with fourfold ribbed handle..$^{57}$ H: 17 (?), W. of handle: $2.2 \mathrm{~cm} .805 / 2.1939$ (Pl. 41).

4. Coins (four items according to the inventory):
(a) Lucius Verus GB (C-XII. 7)
(b) Constantius II PB (C-XII. 8)
(c) 4th century AE 2, badly burned, presumably Constantius II. NH.
(d) PB. 805/1. 1939. NH.

\section{2, ISTVÁN SQUARE (LITTKE BUILDING) (Suppl. 2)}

Grave R/42. This was found on 21 April 1927 in front of the gate of the Littke Champagne Factory,

${ }^{54}$ Gosztonyi $I$.

55 Gosztonyi I, p. 124.

${ }^{56}$ Gy. Fejes, Dunántúl, 25 December 1927; Gosztonyi, op. cit., p. 114, Grave LXXXIV.

57 Isings, p. 159 for further literature. Form 129; Benkö, p. 95 and supra, $4 / \mathrm{b}$ type. in the sewer trench; D: $270 \mathrm{~cm}$. Its roof was made of bricks on their edge $\wedge$. L: $165, W: 50$, internal $\mathrm{H}: 50 \mathrm{~cm} . \mathrm{W}-\mathrm{E}$ orientation. ${ }^{58}$ In the grave there were bones.

Associated articles:

1. Coin Gallenius Ant. (C-XII.9)

2. Coin Gallenius Ant. (C-XII.10)

3. Glass jug. Yellowish-white, thin walled. With flat ribbed handle. H: 16, DM: 5.2, DB: $4.5 \mathrm{~cm} .808 / 1939$ (Pl. 41).

Grave $\mathbf{R} / \mathbf{4 3}$. This was $1.50 \mathrm{~m}$ north of the former grave, D: $260 \mathrm{~cm}$. Its walls were built of bricks $(58 \times 58 \times 8 \mathrm{~cm})$; the inside of the wall was smoothed and had apparently been painted. Roof: $\wedge$, on top of it a $15 \mathrm{~cm}$ thick layer of lime mortar. Inner L: 210, inner W: 110, inner H: $100 \mathrm{~cm}$. Disturbed. ${ }^{59}$ Associated articles:

1. Bones. 20 pieces (A.r. 331/1927.3)

2. Coin. Gallenius Ant. (C-XII. 11)

3. Coin. Gallenius Ant. (C-XII. 12)

New excavations have been conducted on the plot.60

\section{2, ISTVÁN SQUARE (Suppl. 2)}

Graves R/44-46. During the reconstruction of the house three Roman graves were found. D: $200 \mathrm{~cm}$. Built of limestone, with a roof: $\wedge$. Its bottom is of lime mortar. L: 200, inner H: 95, $\mathrm{W}: 85 \mathrm{~cm}$. Side H: $50 \mathrm{~cm}$. W-E orientation. ${ }^{61}$ One male and one female skeleton (heads to the west).

From a disturbed Roman grave at the same place:

1. Coin. Gordian PB four items (A.r. 1932-36. 177/1) NI.

\footnotetext{
${ }^{58}$ Note of Gy. Fejes: JPM-Reference Archives 211; Idem, Dunántúl, 25 December 1927; Gosztonyi I, p. 114.

${ }^{59}$ Cf. Note 58; Gosztonyi I, p. 114, Grave LXXXIII.

${ }^{60} \mathrm{Cf}$. Fülep $V$, the description of the early Christian cemetery excavated between 1968 and 1972 on the site of 12, István Square and 14, Geisler Eta Street.

${ }^{61}$ Gy. Fejes, Dunántúl, 21 July 1931.
} 


\section{5, ISTVÁN SQUARE (Suppl. 2)}

Graves R/47-48. Found in 1926 in front of the house when the main sewer was dug; D: $200 \mathrm{~cm}$. Both were disturbed. ${ }^{62}$

Article:

Glass scent bottle. Green, thin walled. H: 5.9, DB: $3.1 \mathrm{~cm}$. 1143-1939 (Pl. 12, No. 1).

Graves R/49-50. Found on the same site, $60 \mathrm{~cm}$ from the wall of the house; D: $210 \mathrm{~cm}$. Both were disturbed. Human bones and Roman bricks were found. ${ }^{63}$

\section{CORNER OF ISTVÁN SQUARE AND RADONAY STREET (Suppl. 2)}

Graves R/51-53. In 1927 three skeletons were found in yellow sand (A. r. 1927/241).

\section{ISTVÁN SQUARE (Suppl. 2)}

Fragments of a Roman flagon in an undefined courtyard (A. r. 1925/73).

During drainage works Gy. Fejes collected the following articles from unspecified sites:

1. Antefix. Made of reddish clay. Stilized animal head, with red paint on one side and scratched lines around its eye and mouth. ${ }^{64} \mathrm{~L}: 12.5, \mathrm{~W}: 12.9$, Th: $5.1 \mathrm{~cm} .742 / 1939$.

2. Fragment of schola cantorum screen. Yellowish-white, the meeting point of three curving marble ribbons. Broken. L: 18.5, W: 16, Th: $7 \mathrm{~cm}$. 741/1939.

3. Fragment of brick with stamp. In the frame of the stamp: LVPICINITP.P. ${ }^{65}$ (sic!). L: 16.8, W: 11.9, Th: $2.6 \mathrm{~cm} .727 / 1939$.

4. Fragment of roof finial. Brick coloured; with a triangular excision and pierced at several points. Twisted ribbon along one edge. $\mathrm{H}: 14.5, \mathrm{~W}: 10.1$, Th: $3.8 \mathrm{~cm}$. 728/1939 (Pl. 47, No. 1).

${ }^{62}$ Gy. Fejes, Dunántúl, 25 December 1926; Gosztonyi I, p. 111, Graves LVI-LVII.

${ }^{63}$ Fejes, ibid; Gosztonyi I, p. 111, Graves LVIII-LIX.

64 Similar finds: p. 16. Tomb chambers VIII-IX, Inv. No.: 7138; p. 38. Grave R/201.

65 J. Szilágyi: Inscriptiones tegularum Pannonicarum. Diss. Pann. Ser. II No. 1 (Budapest 1933), p. 98. XXVII. No. 34. An officer's stamp of the period of Valentinian I (364-375), ibid, p. 94 .

${ }^{66}$ Opinions differ about its site: According to M. Haas, Baranya Memorial, Pécs 1845, p. 22: it was found in a grave near the cathedral in 21, Scitovszky Square; according to F. Rómer, Arch. Ért. X(1876) p. 176, it was found in 1842 on the land of the pharmacist Nendwitch; later

\section{1, ISTVÁN SQUARE (Suppl.2)}

\section{Grave R/54.}

Remains of a wooden casket ornamented with bronze plates. The front side of the cover is ornamented with struggling centaurs and lions between vine-tendrils in two narrow bands. On the lower portion rectangular panels are arranged in two zones separated by kymatia decoration; there are three panels above and five below. To the left, above, Zeus is surprising Antiope in the shape of a satyr. In the centre there is Leda with the swan. The right side panel is entirely missing, but it might have represented Danae. In the middle of the lower zone the figure of Rome can be seen seated on a throne flanked on two sides by gods of towns indicated by inscriptions: to the left there are Carthage and Constantinople, to the right there are Nicomedia and Siscia. ${ }^{66}$ L: 27, fragmentary $\mathrm{H}$ : $18.5 \mathrm{~cm}$. Its cover is missing. HNM 5/1952.28 (Pls 44-45).

The bronze ringed key in the Horváth collection might have belonged to this. HNM 108/1912.12.

\section{ISTVÁN SQUARE}

Without a more precise definition of the site: Finger ring. With a hemispherical head. D: $1.9 \mathrm{~cm} .1495 / 1939$. M.

\section{JANUS PANNONIUS STREET - THE EARLIER PRIORY OF THE JESUIT ORDER (Suppl. 2)}

Graves R/55-91. During the construction of the Jesuit priory between 1716 and 1726 a painted burial-chamber was found, presumably during the digging of its foundations. ${ }^{67}$

on Rómer learned that it was found below the bishop's palace in the garden of $\mathrm{A}$. Weidinger; Haas, op. cit. p. 228; L. Juhász: Baranya vármegye és Pécs város régészete (The Archaeology of Baranya County and Pécs) Pécs 1894, p. 31. presumes that it was found in 1839 in Apáca Street. In 1852 it was given to the Hungarian National Museum from the Jankovics collection: Baranya II, p. 192; A. Alföldi: Arch. Ért. (1920-22) p. 98; Nagy, p. 141; Gosztonyi I, p. 116; Gosztonyi IV, p. 32; H. Buschhausen: Die spätrömischen Metallscrinia und frühchristlichen Reliquiare. I. Teil. Katalog (Wien 1971), in Wiener Byzantinische Studien, Bd. IX, p. 23 and supra for further literature.

${ }^{67}$ Gosztonyi I, p. 116 for complete literature; Gosztonyi $I V$, p. 31 . 


\section{BURIAL-CHAMBER XI (Suppl. 2)}

Its orientation, dimensions and precise site were not recorded. The earliest source ${ }^{68}$ mentions only that it was found when the foundations of the onetime Jesuit college were built. No mention was made about the nature of the painting of the chamber. In the burial vault there were "a human and a horse skull among other bones". According to 0 . Szőnyi ${ }^{69}$ the inhumation took place as late as the Migration Period, but according to A. Marosi ${ }^{70}$ it dates from the 4th century. O. Szőnyi dealt with the finds again in 1927.71

In our view, which is further proved by results from recent excavations, burial-chambers of Roman date were plundered during the Migration Period and were again used for burials later on..$^{2}$

\section{JANUS PANNONIUS STREET - THE ONE-TIME CISTERCIAN GRAMMAR SCHOOL (Suppl. 2)}

Graves R/92-105. Very little useful information may be recovered from the notes made by Gy. Fejes about the graves found during the excavations in 1934-35. ${ }^{73}$ Here Gy. Fejes found 37 graves altogether. About the graves found in the construction works he notes that they were all at a $\mathrm{D}$. of $465 \mathrm{~cm}$, in contrast to those found during drainage works, which were all at a D. of $220 \mathrm{~cm}$. All were made of brick $^{*}(40 \times 30 \times 5 \mathrm{~cm})$, their roof: $\Lambda$. Alignment exactly $\mathrm{E}-\mathrm{W}$.

The associated articles found in the graves he mentions only summarily: there was a large number of hurnan bones, fragments of pottery, ornamented bronze needles, glass beakers and pieces of iron. During the excavations of 1934 not a single coin was found. He dates the graves to the 4th century.

${ }^{68} \mathrm{G}$. Szerdahelyi: Celebrium Hungariae urbium et oppidorum,Corographia, Cassoviae 1770, p. 230 ... in cryptam fossores incidere picturis intrinsecus variegetam ... Because the quoted work was published in 1770 , several subsequent authors thought the tomb chamber was found in 1770 .

${ }^{69}$ O. Szőnyi: Útmutató a városban, a környéken és a Mecsekben (Directory of the town, its neighbourhood and the Mecsek mountains), Pécs, 2. ed. p. 38

70 A. Marosi, PBMÉ, II (1909) p. 128.

71 O. Szőnyi, Dunántúl, 16 January 1927.

72 Török II, p. 209; Cf. supra pp. 11 and 15; tomb chambers II and VII; in the cemetery at 8, Geisler Eta Street tomb chamber 25 and grave 26: Fülep $I I$, p. 17, Pl. 28.

${ }^{73}$ Notes of Gy. Fejes: JPM-Reference Archives, 215.
In $1935^{74} 14$ Roman graves ${ }^{75}$ were found at a D. of $500 \mathrm{~cm}$ on the Kardos Kálmán Street side. With the exception of four all were disturbed. According to Gy. Fejes these graves differ in type from those found at the same place during drainage works. These graves were all narrower and there were five among them which had no side walls, only a roof. The majority of the graves were at a D. of $500 \mathrm{~cm}$ and he could not exactly define the situation of 8 graves. All the 14 graves were built of brick, their roof: $\wedge$. According to his description, the graves were placed in rows in this part of the cementery. Gy. Fejes dates the graves to the 4th century.76

On the basis of Gy. Fejes's notes and the information in the Pécs Museum we could isolate only four graves:

Grave (a). On the Kardos Kálmán Street side. D: $420 \mathrm{~cm}$, inner L: 125, W: $50 \mathrm{~cm}$. Its bottom was made of mortar. There were bones in the grave, which were those of a child.?7

Grave (b). Its bottom was made of brick, its roof: $\wedge$. L: $125, W: 40 \mathrm{~cm}$. Child's grave. ${ }^{78}$

Articles: Fragment of glass vessel, buckle, earthenware and the button of a brooch. Disintegrated skull.

Grave (e).

Bronze brooch. With three knobs, crossbow type. The central knob is missing, and it is badly corroded. L: (broken) 6.2, W: $4.9 \mathrm{~cm}$. 804-17/1939 (Pl. 11, No. 1).

Grave (d).

Articles:

1. Pottery beaker. Dark grey, coarse, granulated. Double linear ornament on its shoulder. H: 12.7, DM: 11, DB: $5.8 \mathrm{~cm}$. 804/1939.

2. Pottery urn. Vase-shaped, with red paint. H: 15, DM: 7.5, DB: $5.7 \mathrm{~cm}$. 804-2/1939 (Pl. 11, No. 7 )

3. Pottery beaker. With light grey glaze. H: 4.5 , DM: 4.3, DB: $2.4 \mathrm{~cm}$. 804-8/1939.

4. Bronze bracelet. Fragment of a twisted item. 804-12/1939 (Pl. 11, No. 3).

5. Mass-produced lamp. Brown. L: $10 \mathrm{~cm}$. 804-16/1939.

${ }^{74}$ Gy. Fejes, Dunántúl, 24 March 1935.

${ }^{75}$ Gosztonyi $I$, p. 115 . Under No. 34 mentions 35 Roman graves found in 1935: Graves CV-CXL.

${ }^{76}$ Among the finds there are: an altar stone with inscriptions, ef. Stone Finds, Cat. No. 3; further on: yellow and grey clay jugs, glazed jugs, brooches, bronze bangles, buckles, glasses, iron nails, coins, fragments of marble statue and bricks.

77 Notes of Gy. Fejes: JPM-Reference Archives, 215.

${ }^{78}$ Ibid. 
Articles from the remaining graves without any closer description of their origin are the following:

1. Pottery bowl. Dark grey, granulated. H: 7.3, DM: 16.5, DB: 4.3 cm. 804-3/1939 (Pl. 11, No. 9).

2. Pottery beaker. Dark grey, granulated. $\mathrm{H}$ : 10.3, DM: 8.7, DB: $4.3 \mathrm{~cm}$. 804-4/1939 (Pl. 11, No. 8).

3. Pottery beaker with a handle. Dark grey, fine surfaced. With double ribbed handle. Complete. H: 8.7, DM: 7.1, DB: 4 cm. 804-5/1939 (Pl. 11, No. 6).

4. Crucible. Dark grey, granulated. Of pierced, burned clay. Its mouth is triangular. H: 7, DM: 6.9, DB: 4.3 cm. 804-6/1939.

5. Pottery beaker. Light brown, soot-marked on its side. Worn. H: 4.9, DM: 4, DB: $3.5 \mathrm{~cm}$. 804-7/1939.

6. Pottery bowl. Brick-coloured, roughly granulated. Inside are traces of green glaze. H: 3.2, DM: 10.6, DB: 4.4 cm. 804-9/1939 (Pl. 11, No. 10).

7. Bronze buckle. Oval, with rectangular tongue. W: $3.9 \mathrm{~cm}$. 804-10/1939. M.

8. Bronze bracelet. Thin, made of wire with D-shaped cross section. Penannular, with snakeheads at its ends. D: 6.1, W: 0.3 , Th: $0.2 \mathrm{~cm}$. 804-11/1939.

9. Same. (Description as above.) D: 6.1, Th: $0.2 \mathrm{~cm} .804-12 / 1939$. M.

10. Same. Made of thin, cylindrical wire, open. Snake-heads on its flattened ends. D: 4.9, Th: $0.3 \mathrm{~cm}$. 804-13/1939.

11. Bronze buckle. Oblong in shape, with wedgeshaped notches. L: $3, \mathrm{~W}: 1.9$, Th: $0.3 \mathrm{~cm}$. 804-14/1939 (Pl. 11, No. 2).

12. Small glass bottle. Greenish-blue, thick walled. Intact. H: 8.1, DM: 2.5, DB: $3.1 \mathrm{~cm}$. 804-19/1939 (Pl. 11, No. 5).

13. Bronze brooch. Three-knobbed, crossbow type. Ornamented foot. L: 8.9, W: $6.2 \mathrm{~cm}$. 2916/1941.

14. Same. Crossbow brooch, broken. Decorated with four and six punched ringlets on its foot, with a safety device. L: $3.7 \mathrm{~cm}$. 2917/1941.

15. Altar stone. ${ }^{79}$

16. Bronze bracelet. Of thin wire, broken. D: $4.7 \mathrm{~cm} .2912$. M.

17. Fragments of bronze vessel. Five pieces. 2902. M.

18. Iron knife. Flat, its point is broken. L: 25 , W: 3.5, Th: $0.3 \mathrm{~cm} .2919 / 1941$.

19. Bronze cauldron. Fragments of side and handle. The handle is in the shape of a swan's head, inscription IVV on it. ${ }^{80}$ L: 24, Th: $0.9 \mathrm{~cm} .2922 / 1941$ (Pl. 4 \%, No. 3 ).

\footnotetext{
79 Stone Finds, Cat. No. 3.

${ }^{80}$ Radnóti $I, \mathrm{XXXV}$, No. 7; Intercisa $I I$, p. 183 and supra, XXXVII. No. 1; XXXVIII. No. 2; XXXIX. No. 7, Cat. No. 15, 217.
}

20. Bronze bracelet. Twisted from four wires, with a hooked clasp. Broken into three pieces. D: 6.5, Th: $0.8 \mathrm{~cm} .927$ (Pl. 11, No. 4).

21. Coins. Five items. PB. 2924-2928/1941. NI.

\section{3, JANUS PANNONIUS STREET (Suppl. 2)}

Grave R/106. This was found in 1927 during drainage works $18 \mathrm{~m}$ west of the corner of Leonardo da Vinci (Szepessy) Street. Brick grave, its roof: $\wedge$; disturbed. ${ }^{81}$

Articles:

Human bones, green glazed fragments of vessels, pieces of bronze chains, one bronze ornamental piece.

\section{3, JANUS PANNONIUS STREET (Suppl. 2)}

Grave $\mathbf{R} / \mathbf{1 0 7}$. In the garden of the house, a brick grave. Its side walls were built of limestone. Th. of wall: $15 \mathrm{~cm}$. D: $465 \mathrm{~cm}, \mathrm{~L}: 200, \mathrm{~W}: 68, \mathrm{H}: 70 \mathrm{~cm}$. Its roof: $\wedge$. E-W orientation, disturbed. ${ }^{82}$

\section{5, JANUS PANNONIUS STREET (Suppl. 2)}

Grave $\mathbf{R} / \mathbf{1 0 8}$. At the corner of Székesfehérvár (Vörösmarty) Street, in front of the entrance of the former Cseh Palace. It was found in 1927 during drainage works. D: $140 \mathrm{~cm} .^{83}$

Articles:

1. Bronze bowl. Fragment of the bottom of a bigger vessel, made in one piece. D: 9.8, H: 1.6, Th: $0.1 \mathrm{~cm} .814-4 / 1939$ ( $P l .12$, No. 5).

2. Mass-produced lamp. Light brown, one big and one small hole in its cover-plate. Framed inscription on its bottom: VIBIANI. L: 8.5, H: 2.8 , DB: $2.8 \mathrm{~cm} .814 / 1939$.

3. Same. Cream-coloured; with two knobs. On its bottom in a double circle: FORTIS. With traces of orange coloured paint. L: $9.3, \mathrm{H}: 2.9 \mathrm{~cm}$. 814-2/1939 ( $P l .12$, No. 3).

4. Same. Brick-coloured, its cover-plate is broken; with three knobs. L: 6.3 , H: $2.9 \mathrm{~cm}$. 814-3/1939 (Pl. 12, No. 2).

\section{5, JANUS PANNONIUS STREET (Suppl. 2)}

By way of donation, the Pécs Museum obtained (from the land of the former Cseh Palace) the following articles in 1908:

\footnotetext{
81 Notes of Gy. Fejes: JPM-Reference Archives, 222; Idem. Dunántúl, 25 December 1927.

82 Gy. Fejes, Dunántúl, 4 November 1934.

${ }^{83}$ Gy. Fejes, Dunántúl, 25 December 1927.
} 
1. Inscribed stone. ${ }^{84}$

2. Fragment of tombstone. ${ }^{85}$

3. Fragment of sarcophagus. ${ }^{86}$

\section{4, JANUS PANNONIUS STREET (Suppl. 2)}

Graves R/109-111. In 1927 during canalization works one disturbed Roman grave was found at the entrance of the house, and two more were discovered east of it. D: $230 \mathrm{~cm} .^{87}$

\section{Grave R/109.}

Articles:

1. Pottery bowl. Cream-coloured, rough precipitation. Complete. H: 5, DM: 23, DB: $9.5 \mathrm{~cm}$. 1990. (Pl. 12, No. 4.)

\section{Grave R/110.}

Articles:

1. Bronze weight. Solid. Shaped as an octahedron. D: $1.7 \mathrm{~cm} .1118 / 1939$.

2. Bronze die. Same. D: 1.4 cm. 812/1939.

\section{Grave R/111.}

Articles:

1. Base of a pottery flagon. Grey. (A. r. 1927/337-39.)

\section{4, JANUS PANNONIUS STREET (Suppl. 2)}

In front of the staircase, among ruinous walls of uncertain age, together with a few bones. D: $120 \mathrm{~cm}$.

1. Fragment of earthen vessel. Thin-walled, yellow. With scratched on curvilinear decoration, and red paint. Sooted. 7191. M.

\section{1, JANUS PANNONIUS STREET (Suppl. 2)}

In 1960 we conducted excavations on this plot and found the following Roman articles.

Northern part of Trench XX, D: between 315-325 cm:

1. Fragment of pot. Grey, the side and bottom fragment of a big vessel. With greenish-yellow glaze outside and inside. 61.144.19.

Trench XX, from a pit, D: $325-505 \mathrm{~cm}$ :

84 Stone Finds, Cat. No. 70.

85 Ibid., Cat. No. 22.

${ }^{86}$ Ibid., Cat. No. 65 .

87 Gy. Fejes, Dunántúl, 25 December 1927; Gosztonyi I, p. 114, Graves LXXXV-LXXXVII.
1. Fragment of pot. Yellow, made of finely granulated clay. Reddish-brown, with painted lines and dotted pattern. 61.144.23.

Trench XXVI, D: 190-260 cm:

1. Coin. Julian AE3 (C-XVI. 2).

\section{GEISLER ETA (APÁCA) STREET (Suppl. 2)}

Grave R/112. Without a more closely defined location; a brick grave. Its finds were obtained by the HNM by donation:88

1. Bronze ring. Flat, slightly protruding around the middle. Concave on its reverse. Complete, with attractive patina. D: 7.1, W: 1.5 , Th: $0.4 \mathrm{~cm}$. HNM 298/1874 ( $\mathrm{Pl}$. 12, No. 7).

2. Bronze brooch. Three-knobbed, crossbow brooch. With safety device, its pin fragmentary. L: 9.3, W: 5.8 cm. HNM 108/1912.47 (Pl. 12, No. 6).

\section{4, GEISLER ETA STREET (Suppl. 2)}

Grave R/113. This was found by $O$. Szőnyi east of the entrance in the courtyard. ${ }^{89} \mathrm{D}: 220 \mathrm{~cm}$. Earth grave, without side walls. Its roof was made of five pairs of bricks: $\wedge$. On its ridge were imbrices. L: $190 \mathrm{~cm}$. E-W orientation. It had no associated articles.

\section{6, GEISLER ETA STREET (Suppl. 2)}

Grave R/114. Found at the entrance of the local branch of the Hungarian National Bank. D: $200 \mathrm{~cm}$.

\section{Articles:}

1. Fragments of glass beaker. Yellowish-green, thin walled. Broken. DB: $3.8 \mathrm{~cm} .767 / 1939$.

2. Fragments of glass beaker. Thin walled. 766/1939. M.

3. Roman brick. Carved. (A. r. 1925/43). N. I.

\section{6, GEISLER ETA STREET (Suppl. 2)}

Grave R/115. A grave with a skeleton found in 1938 during the construction of the local headquarters of the Hungarian National Bank. The grave was not described.

${ }^{88}$ T. Ortvay, Arch. Közl. X(1876) p. 128; Baranya II, p. 195.

${ }^{89}$ O. Szőnyi, PBMÉ (1914) p. 91; Gosztonyi I, p. 110, Grave LIII. 
Articles:

1. Glass bracelet. Of black glass. D-shaped cross section, closed. Broken into three pieces. Diam: $6.7 \mathrm{~cm}$. 7095/1941.

2. Fragments of bronze bracelet. Two pieces. Made of thin, flat wire, with tiny cuts on its side. One tapered end is twisted around the other. Broken. Diam: 6.6, W: $0.3 \mathrm{~cm}$. 7095/1941.

\section{7, GEISLER ETA STREET (Suppl. 2)}

Grave $\mathbf{R} / 116$. This was found during drainage works. D: $200 \mathrm{~cm}$, disturbed. ${ }^{90}$ Inside were a human skull and other bones. (A. r. 182/1927. 46).

\section{8, GEISLER ETA STREET (Suppl. 2)}

Grave R/117. This was found in front of the house below the road surface in $1926 .{ }^{91}$ Brick grave. D: 50, L: 142 , W: $40 \mathrm{~cm}$. D. of grave: $35 \mathrm{~cm}$. Child's grave. No articles.

In 1927 Gy. Fejes found the following at a D. of $190 \mathrm{~cm}$ during drainage works:

1. Bronze bracelet. Made of wire of D-shaped cross section. Open, with stylized snake-heads. Complete. 813/1939.

2. Coin: Constantine I. Foll. (C-XII. 13)

A description of the cemetery excavated between 1958 and 1961 is given elsewhere. ${ }^{92}$

\section{0, GEISLER ETA STREET (Suppl. 2)}

Graves R/118-124. During reconstruction works several Roman brick graves were found $30 \mathrm{~cm}$ below the floor. Side walls were built of brick and limestone, roof: $\Lambda,(60 \times 60 \times 9 \mathrm{~cm}$ bricks $), H$ : $56 \mathrm{~cm}$. According to Gy. Fejes ${ }^{93}$ the dimensions of one of the graves were: inner L: 196, W: 70, H: $101 \mathrm{~cm}$. Nothing is known about orientation.

They were disturbed and contained bones and fragments of vessels. Gy. Fejes mentions that in front of house No. 10 four complete Roman graves were found in 1927. He unearthed 23 graves altogether in Apáca Street with rich finds. According to his estimate they are brick graves from the 3rd century.

\footnotetext{
${ }^{90}$ Gosztonyi I, p. 114, Grave LXXXII.

91 Dunántúl, 13 August 1926; Gosztonyi, I, p. 110, Grave LIV.

92 Fïlep $I I$, p. 3 and supra.

${ }^{93}$ Gy. Fejes, Dunántúl, 28 March 1934.
}

\section{4, GEISLER ETA STREET (THE FORMER CZIERER BUILDING)}

(Suppl. 2)

Graves R/125-145. In the rear of the courtyard a disturbed Roman grave was found in 1906. ${ }^{94}$ Its walls were of limestone, its roof of four pairs of brick: $\wedge$. The bottom of the grave is also made of bricks. Inner L: 185, W: $63, \mathrm{H}: 82 \mathrm{~cm}$. No associated articles.

In the courtyard 20 Roman graves were found in 1932. D: 70-100 cm. According to Gy. Fejes ${ }^{95}$ the graves were of $\mathrm{N}-\mathrm{S}$ orientation and placed closely one by the other. There were 6 children's and 14 adult skeletons. Inner $\mathrm{L}$. of the adult graves: 180-200, W: 45, $\mathrm{H}$ (together with the roof): $70-80 \mathrm{~cm}$. The L. of one grave was $240 \mathrm{~cm}$. Graves 6 and 7 were built adjoining, and were presumably double graves. Inner L. of children's graves: 120-160 cm. According to the description by Gy. Fejes, six were earth graves, their roof: $\Lambda$. Orientation: W-E (head to the west). Fragments: pieces of pottery, glass beakers, bronze pieces; in four graves there were illegible, worn coins around the skull. These graves are dated by Gy. Fejes approximately to the 3rd century.

Articles:

1. Fragments of glass beakers. Ten pieces (A. r. 306/1932). Of these:

Fragments of glass beaker. Two pieces. Small, white, thin walled. 2224/1940.

2. Roman brick. With cursive writing on one side. ${ }^{96}$ Broken into three pieces. L: 37.9, W: 25.5, H: $5.6 \mathrm{~cm}$. 731/1939. (Pl. 48, Fig. 1).

3. Roman brick. With an incised figure of a stag..$^{9 ?}$ Broken. L (fragmentary): 32.5, W: 29.5, H: $5.7 \mathrm{~cm}$. 730/1939 (Pl. 15, No. 1).

At the beginning of 1968 when water pipes were laid down, eight Roman brick graves were found; excavations followed in the courtyard in the summer of $1969 .{ }^{98}$

\section{3, GEISLER ETA STREET (Suppl. 2)}

Grave R/146. In 1870, when gas pipes were laid down this grave was found below the road surface, at the NE corner of the building of the convent.

\footnotetext{
${ }^{94}$ O. Szőnyi, $P B M E ́$ VII (1914) p. 91; Gosztonyi $I$, p. 107.

${ }^{95}$ Gy. Fejes, Dunántúl, 24 April and 18 August 1932.

${ }^{96}$ See in detail: Frülep $V$.

${ }^{97}$ Cf. Kuzsinszky, p. 70, Fig. 90. From the cemetery of Fenék, from a late Roman grave (head to the W). ${ }^{98} \mathrm{Cf}$. footnote 60 .
} 


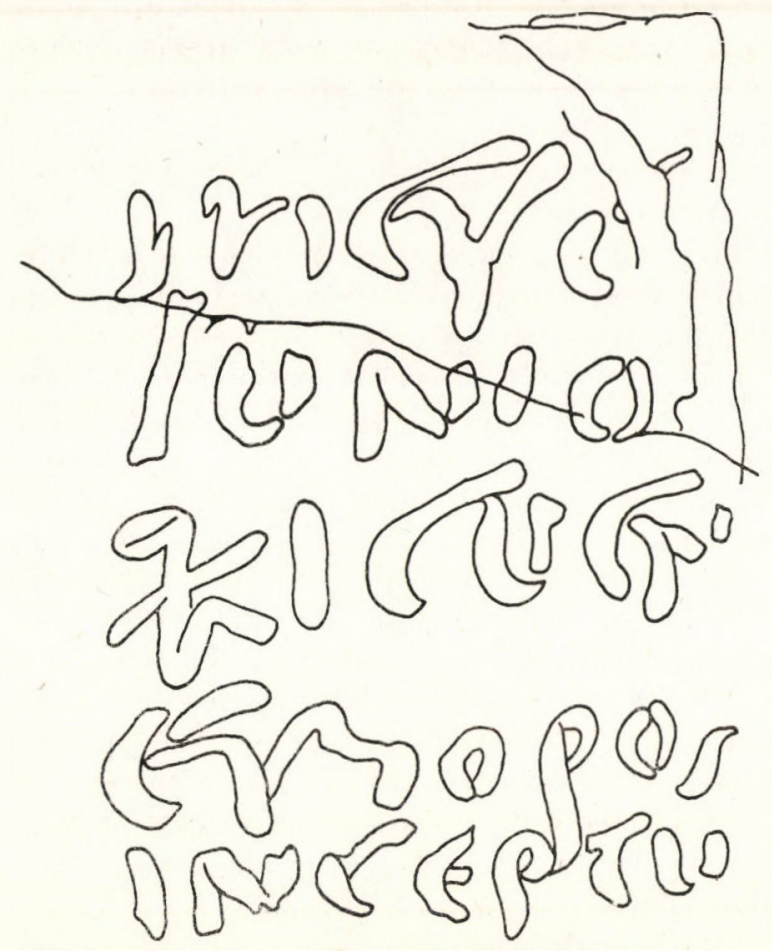

Fig. 1 Roman brick

D: ca. $90 \mathrm{~cm}$. The walls of the graves were built of stone and brick, roof: $\wedge .{ }^{99} \mathrm{E}-\mathrm{W}$ orientation.

Articles:

1. Glass bracelet. Dark blue, D-shaped cross section. Complete. Diam: $7, \mathrm{~W}: 0.6$, Th: $0.5 \mathrm{~cm}$. 762/1939 (Pl. 15, No. 2).

2. Coins. Two items. Constantine II(?). NI.

Grave R/147. This was found in 1927 during drainage works $5 \mathrm{~m}$ from the pit in front of the convent; disturbed. ${ }^{100}$ D: $190 \mathrm{~cm}$. Inside were pieces of brick and bones.

Articles:

1. Coin: Probus Ant. (C-XII. 14).

2. Coin: Probus Ant. (C-XII. 15).

$16 \mathrm{~m}$ from this grave were two other disturbed graves:

\section{Grave R/148.}

Articles:

1. Coin: Probus Ant. (C-XII. 16). Altogether Gy. Fejes mentions six coins of Probus in his notes.

99 A. Horváth, Arch. Ért. (1870) p. 122; Baranya II, p. 194; Gosztonyi I, p. 106, Grave XVII.

100 Notes of Gy. Fejes: JPM-Reference Archives, 212; Idem., Dunántúl, 25 December 1927; Gosztonyi I, p. 113, Graves LXXVII-LXXIX.
Grave R/149. Coins of "Constantinus Chlorus" in the grave, ${ }^{101}$ but their number is not specified. The following could be identified:

1. Coin: Constantine I. Foll. (C-XII. 17).

2. Coin: Constantine I. Foll. (C-XII. 18).

\section{GEISLER ETA STREET (Suppl. 2)}

Grave R/150. This was found in 1927 during drainage works at the eastern wall of the convent building; disturbed.

Articles:

1. Coin: Licinius Foll. (C-XII. 19). ${ }^{102}$

2. Coin: Constantius II AE 2 (C-XII. 20). ${ }^{103}$

\section{GEISLER ETA STREET, CONVENT BUILDING (Suppl. 2)}

Graves R/151-163. In 1935, during the extension of the convent building, twelve graves were found.

According to the notes of Gy. Fejes ${ }^{104}$ the D. of one of them is $140 \mathrm{~cm}$. This was an earth grave, roof: $\wedge$. L. of one of the graves: $70, H: 40 \mathrm{~cm}$. No other information is given.

Associated articles:105

1. Fragment of wall paintings. 14 items. 816-1/1939. M.

2. Bronze belt fitting. Elongated leaf shape, with two pelta motifs cut out. L: 4.9, W: 2.2, Th: 0.2-0.4 cm. 816-2/1939 (Pl. 13, No. 8).

3. Same as above. Heart-shaped, with four dotted circle motifs. L: $3.1, \mathrm{~W}: 2.8$, Th: $0.1-0.3 \mathrm{~cm}$. 816-3/1939 (Pl. 13, No. 9).

4. Pierced bronze mount. Irregular, approximately rectangular, with round pierced holes. Belt plate (?). L: $2.6, \mathrm{~W}: 2.6$, Th: $0.1 \mathrm{~cm} .816-4 / 1939$.

5. Bronze mount. Solid, with pelta decoration. From a horse harness (?). L: 5.5, W: 5.2, Th: 0.4-0.7 cm. 816-5/1939 (Pl. 13, No. 10).

6. Bronze ring. Solid, of circular cross section. Diam: 7.5, W: 0.8, Th: $0.8 \mathrm{~cm} .816-6 / 1939$.

7. Jet bracelet. Smooth, D-shaped cross section. Diam: 7.9, W: 0.7, Th: $0.5 \mathrm{~cm}$. 816-7/1939 (Pl. 14, No. 2).

8. Bronze bracelet. Tapering toward the ends,

101 Gy. Fejes, Dunántúl, 25 December 1927.

102 Fejes, p. 44, No. 324, Fig. 6.

${ }^{103}$ Ibid. p. 47, No. 388. Fig. 7; A. Sz. Burger, JPMÉ XVI (1972) p. 117, No. 257.

104 Notes of Gy. Fejes: JPM-Reference Archives, 203; Gosztonyi I, Graves XCIII-CXIV.

105 , István Square and Scitovszky Square are indicated as sites. 
open, with densely incised lines at the ends. Diam: $7.5 \mathrm{~cm} .816-8 / 1939$. M.

9. Fragments of twisted bracelet. Twisted of three wires, with hook and loop fastening. Broken into two pieces. Two items. Diam: 6.2, Th: $0.2 \mathrm{~cm}$. 816-9/1939 (Pl. 14, Nos 3-4).

10. Bronze bracelet. Of D-shaped cross section, solid. Penannular, ending in collared snake-heads. One end is broken. Diam: $5.9, \mathrm{~W}: 0.6$, Th: $0.4 \mathrm{~cm}$. 816-10/1939.

11. Bronze buckle. Of rectangular cross section, a so-called "Omega-buckle". With movable tongue. Diam: 4.7, Th: $0.3 \mathrm{~cm}$. L. of pin: $4.9, \mathrm{~W}: 0.3 \mathrm{~cm}$. 816-13/1939. (Pl. 13, No. 5).

12. Bronze bracelet. The tapered ends of the wire are wound around one another several times. Complete. Diam: 5, Th: 0.3 cm. 816-12/1939 (Pl. 14, No. 1).

13. Bronze buckle. Oval, fracted at the edge. With a movable tongue ornamented with elaborated animal head. The two ends of the plate attached to the belt are indented on the edge and have dot-and-circle decoration. Broken. L. of loop: 3.7, W: 2, Th: $0.4 \mathrm{~cm}$. L. of belt plate: $3.1, \mathrm{~W}: 3.1 \mathrm{~cm}$, Th: $0.3 \mathrm{~cm} .816-14 / 1939$ (Pl. 13, No. 7).

14. Same as above. D-shaped, with movable tongue. L. of buckle: $3.6, \mathrm{~W}: 2 \mathrm{~cm}$. L. of belt plate: 2.4, W: $2.1 \mathrm{~cm}$. 816-11/1939 (Pl. 13, No. 6).

15. Bronze brooch. Three-knobbed, crossbow brooch. Solid, pin is missing. L: $5.4, \mathrm{~W}: 3.9 \mathrm{~cm}$. 816-15/1939 (Pl. 13, No. 1).

16. Bronze bracelet. Of solid wire with D cross section. Open, collared snake-heads at the ends. Diam: 5.9, W: 0.6, Th: $0.4 \mathrm{~cm}$. 816-16/1939.

17. Spindle-whorl, of reddish-brown fabric, plain. Diam: 2.7, H: 1.8 cm. 816-17/1939 (Pl. 14, No. 5).

18. Spindle-whorl, of light brown fabric, ribbed several times. Diam: 3.2, H: $2 \mathrm{~cm} .816-18 / 1939$ (Pl. 14, No. 6).

19. Samian bowl. Fragments of type Drag. 37. I'wo pieces. 817-1/1939. M.

20. Bronze brooch. Three-knobbed, crossbow brooch. The knobs are faceted. Pin is missing. L: 7.1, W: 5, Th: $0.3 \mathrm{~cm}$. 818-3/1939 (Pl. 13, No. 4).

21. Bronze brooch. Three-knobbed, crossbow brooch. Pin missing. L: $6.9, \mathrm{~W}: 4.1$, Th: $0.7 \mathrm{~cm}$. 818-4/1939 (Pl. 13, No. 2).

22. Pottery lamp. Light grey mass-produced lamp with three knobs. One hole between the coverplate and nozzle. L: 8.5, H: $3, \mathrm{~W}: 5.8 \mathrm{~cm} .818-5 / 1939$.

23. Bronze fitting. Handle with oblong cross section; one end bends upwards in an arch, and one oval disc is set directly below another which shows the stump of a broken extension. Solid. L: 5.9, Th: 1.5, Diam. of button: $3.7 \mathrm{~cm}$. 818-6/1939 ( $P l$. 13, No. 11).
24. Glass beads. 70 items. 818-7/1939. M.

25. Fragments of glass beaker. Greenish-yellow, thin-walled. Diam: $4.6 \mathrm{~cm}$. 818-8.e/1939. (Pl. 41, No. 4).

26. Same as above. Yellowish-white, thin-walled. DM: $7.3 \mathrm{~cm} .818-8 / 1939$.

27. Fragments of glass beaker. Greenish-yellow, thin-walled. DB: 4 cm. 818-8.d./1939 (Pl. 41, No. 3).

28. Same as above. Yellowish-white, thin-walled, fragments of side and bottom. DB: $5.1 \mathrm{~cm}$. 818-8/1939.

29. Glass beaker. Yellowish-white, of thicker wall. Parts are missing from the body. H: 8.6, DB: 5 cm. 818-8.f/1939. (Pl. 14, No. 8; Pl. 41, No. 7).

30. Glass bottle. Yellowish-white, thin-walled. 818-8.b/1939 (Pl. 14, No. 7; Pl. 41, No. 2).

31. Fragments of glass beaker. Greenish-white, thin-walled. DB: 4.3 cm. 818-8.g/1939 (Pl. 41, No. 8).

32. Fragment of glass vessel. Greenish-white, fragment of the mouth of a thin-walled oinochoe. DM: $5.3 \mathrm{~cm} .818-8 . \mathrm{a} / 1939$ (Pl. 41, No. 1).

33. Fragment of glass vessel. Yellowish-white, thin-walled. Fragment of a rhyton (?). L: $7.2 \mathrm{~cm}$. 818-8.i/1939 (Pl. 41, No. 6).

34. Fragments of glass bottle. Greenish-blue, thick-walled. DB: 6.6 cm. 818-8.c/1939 (Pl. 41, No. 5).

35. Fragment of glass flagon (?). Greenish-yellow, thin-walled, with engraved band. H (fragmented): 9 , DB: $5.5 \mathrm{~cm}$. 818-8.h/1939 (Pl. 41, No. 9).

36. Glass button. Made of bluish-grey glass paste; oval shaped. Flat at the bottom and hemispherical on top. From a ring (?). Complete. L: 1.4, W: 1.2, Th: $0.6 \mathrm{~cm} .818-9 / 1939$.

37. Bone article. Cream-coloured, flat, one side has been pierced. L: $3.3, \mathrm{~W}: 0.7$, Th: $0.3 \mathrm{~cm}$. 818-10/1939.

\section{GEISLER ETA STREET}

The following object comes from a Roman grave without exact location:

Handled flagon (from the collection of A. Horváth) (A. r. 1912/528).

\section{CORNER OF GEISLER ETA STREET AND SZÉKESFEHÉRVÁR (VÖRÖSMARTY) STREET (TALLIÁN SITE) (Suppl. 2)}

Graves R/164-176. In 1910 while the foundations were being dug for the National Bank building 
13 graves altogether were found. ${ }^{106}$ Of these O. Szőnyi could properly excavate two only.

Grave R/164. Given as gifts to the Pécs Museum:

$\begin{array}{lll}\text { 1. Coin: Numerianus NA. } & \\ \text { 2. Coin: Probus } & \text { NA. }\end{array}$

Grave R/165. Gift of A. Horváth, from the grave of a girl according to O. Szőnyi: ${ }^{107}$

1. Pottery bowl. Brick-red, with dark green glaze on its inside. H: 5, DM: 11.1, DB: $4.8 \mathrm{~cm}$. 793-2/1939 (Pl. 15, No. 3).

2. Bronze ring. NI.

3. Small wooden cylinder with screw. NI.

4. Bone bead. Barrel-shaped. Light yellow, pierced. L: 2.1, Diam: 2.3 cm. 793-1/1939 (Pl. 15, No. 4).

5. Glass bead. (A. r. 1910/461) NI.

Grave R/166. Donated by A. Horváth; according to $O$. Szónyi ${ }^{108}$ the finds were found in a male grave:

1. Coins. Three items. One is $\mathrm{GB}$, one is $\mathrm{MB}$ and one is Ant. (IMP C CLAV . . .). 6151-53/1940. M.

2. Bronze buckle. D-shaped. The belt plate is broken. H. of loop: $3, \mathrm{~W}: 2$, Th: $0.4 \mathrm{~cm}$. H. of plate: 3, W: 2.9, Th: 0.5 cm. 794-2/1939 (Pl. 15, No. 5).

3. Small bronze strip. Narrow and flat, ornamented with incised lines. One nail in its end. Broken. L: 4.8, W: 1, Th: $0.5 \mathrm{~cm}$. 794-1/1939 (Pl. 15, No. 6).

Grave $\mathbf{R} / \mathbf{1 6 7}$. The following were given to the museum as gifts: 109

1. Pottery flagon. With dark green glaze, mouth broken. H: 22, DM: 4.2 , DB: $7.3 \mathrm{~cm}$. 795-1/1939 (Pl. 15, No. 8).

2. Fragments of vessel. 27 pieces. 795-2/1939.

3. Fragments of bronze vessel. 18 pieces. NI.

4. Glass beads. Six items. NI.

106 O. Szőnyi, $P B M E ́$ (1910) p. 122 and supra. Here Szőnyi mentions 13 graves. On the other hand Szőnyi himself mentions only 12 graves in the A. r. of the Pécs Museum: A. r. 1910. 168 (and under serial No. 1957. from a-h 466); Idem. PBMÉ (1911) p. 19. Here again 13 graves are mentioned; Gosztonyi $I$, p. 108, Graves XXXIII-XLIII: these are 11 altogether. This number corresponds to the number of graves indicated in the drawings of Flórián Cséfay, the town engineer (the drawings are lost).

107 O. Szőnyi, PBMÉ (1910) p. 122. Besides the finds enumerated in our paper here Szónyi also mentions two hairpins.

108 O. Szőnyi, ibid.

109 O. Szőnyi, ibid.
5. Fragments of glass bracelet. Dark blue, of D-shaped cross section, worn. Two pieces. Diam: 6.3, W: 0.8 , Th: $0.5 \mathrm{~cm}$. 795-3/1939.

6. Coins. Five items. 4 th cent. PB. 4170-74/1939. NI.

Grave R/168. The following were given as gifts to the Pécs Museum on 19 September 1910:

1. Bronze knee brooch. Smooth, plain. Pin is broken. L: 4.1, W: 1.3, Th: $0.5 \mathrm{~cm} .795-4 / 1939$ (Pl. 16, No. 2).

2. Bronze brooch. Three-knobbed, crossbow brooch. Twisted bronze wire at the base of the knobs; the bow also rests on twisted wire. Complete, pin intact. L: 7.3 , W: 4.9, Th: $0.5 \mathrm{~cm} .795-5 / 1939$ (Pl. 16, No. 4).

3. Same as above. Three-knobbed, crossbow brooch. The knobs are solid, ribbed with twisted bronze wire at the base of each. Small, ribbed strip at the base of the bow as well. Intact, pin broken. L: 8.1, W: 6.5, Th: $0.7 \mathrm{~cm}$. 795-6/1939 (Pl. 16, No. 3).

4. Bronze disc. Thin, slightly embossed with a schematic representation of a female head. On the basis of the profuse hair it may be interpreted as a representation of a Medusa head (?); it once belonged to a scrinium. ${ }^{110}$ Diam: 5.4 , Th: $0.1 \mathrm{~cm}$. 795-7/1939 (Pl. 16, No. 1).

5. Iron objects. Six items (five are extant):

(a) Fragment of iron hammer. Broken; woodfibre in the hole of the shaft. L: 7.2, W: 1.7, Th: 0.3-1.4 cm. 795-9/1939.

(b) Iron object. Of rectangular cross section, ending in a sharp edge. Broken. Goldsmith's tool (?). L: 12.2, W: $1.3 \mathrm{~cm}$. 795-10/1939 (Pl. 16, No. 6).

(c) Iron bar. Long, thin, one end is rounded. L: 9.2, W: 1.5 , Th: $0.5 \mathrm{~cm}$. 795-11/1939.

(d) Fragment of iron knife. Single edged, leafshaped, with traces of wood-fibre. L: 12, W: 3.3, Th: 0.4-1 cm. 795-8/1939 ( $P l .16$, No. 7).

(e) Iron object. Of rectangular cross section. Goldsmith's tool (?), may be related to (b). L: 16.5, W: 3.2, Th: 0.3-2.2 cm. 795-15/1939 (Pl. 16, No. 5).

6. Pottery flagon. Light brown, of granulated clay. Fragment of handle on its side. Hand-made, sooted on the outside. H: $14.3, \mathrm{DM}: 6.3, \mathrm{DB}: 8.5 \mathrm{~cm}$. 795-12/1939 (Pl. 16, No. 9).

7. Jet bangle. Of prismatic cross section, the inner side is rounded, the outer octagonal, black. Split into plates. Outer rim with motif cut three times. Diam: 8.7, Th: 0.9 cm. 795-14/1939 (Pl. 16 . No. 8).

110 Cf. R. M. Alföldi: Intercisa II, Figs 65, 69, 83. 
Grave $\mathbf{R} / \mathbf{1 6 9}$. At the western edge of the plot; brick grave. 111 D: $150 \mathrm{~cm}$. Inner L: $200, \mathrm{~W}: 58 \mathrm{~cm}$. H. of walls: $30 \mathrm{~cm}$. E-W orientation. Female skeleton in the grave.

Associated articles:

1. Gold earrings. Two items. With blue glass (at the head). NI.

2. Gold ornaments. Three items. From a necklace. Weight: 1.43 g. 796-1/1939.

3. Glass beads. 24 items. Small size. 796-2/1939. M.

4. Bronze bracelet. Twisted of two wires, broken. Diam: $6.1 \mathrm{~cm} .796-3 / 1939$.

5. Bone hairpin. Cylindrical, plain. Both ends are broken. L: 6.3 , Th: $0.2-0.5 \mathrm{~cm}$. (According to the Acquisitions register it is a "wooden pin".) 796-4/1939 (Pl. 26, No. 2).

6. Pottery flagon. Cream-coloured, fine surface. With a wide stripe of orange-red paint on the outer surface. Intact (at the feet of the skeleton). H: 23.4, DM: 8, DB: $7.5 \mathrm{~cm}$. 796-5/1939 (Pl. 15 . No. 7).

7. "Copper coin" (at the head). NI.

Grave $\mathbf{R} / \mathbf{1 7 0} .2 \mathrm{~m}$ north of the earlier grave; size and construction are similar, two rows of bricks on top.

According to O. Szőnyi112 there was a male skeleton in the grave, but orientation is not indicated.

Associated articles:

1. Coins:

(a) Probus Ant. (C-XII. 22) (Pl. 17, No. 16)

(b) Probus Ant. (C-XII. 23) (Pl. 1\%, No. 18)

(c) Carinus Ant. (C-XII. 24) (Pl. 17, No. 17)

2. Fragments of glass bottle. Greenish-white, thinwalled, with a glass filament running around the body of the bottle. Completely broken. H: 13.5, DM: $4.8 \mathrm{~cm}$. 797-12/1939 (Pl. 41, No. 1).

2a. Same as above. Greenish-white, thin-walled. Its bulbous body is ornamented with a glass filament twisting around it several times. Completely broken. DM: $6 \mathrm{~cm}$. 797-12/1939 (Pl. 41, No. 2).

3. Silver brooch. (According to the Acquisitions register this is a bronze brooch with a bow.) Threeknobbed, crossbow type. The cross bar and the three knobs are of bronze, the bow is of silver; the

111 O. Szőnyi, $P B M E$ (1910) p. 123: A colour drawing done by Szőnyi on 12 August 1910 is extant and represents one of the graves found here. Szőnyi mentions in several places that of the graves found here two were barrel vaulted and the rest had gabled roof. Unfortunately it could not be aseertained which of the graves with gabled roof was drawn by Szőnyi; Gosztonyi I, p. 108, Grave XXXI.

112 O. Szőnyi, PBME (1910) p. 123; Gosztonyi $I, \mathrm{p}$ 108, Grave XXXII. hexagonal cross-piece is also covered by silver plate. Central knob pegged. Broken, pin missing. L: 6.1, W: 5.1, Th: $0.5 \mathrm{~cm}$. 797-3/1939 (Pl. 17, No. 2).

4. Bronze plate. Circular, with thickened edge. Diam: 27, Th: $0.2 \mathrm{~cm}$. 797-4/1939 (Pl. 17, No. 4).

4a. Bronze nails. Six items. With flat head, one ends in a ring. L: $1.8,3-3.1 \mathrm{~cm}$. 797-4/1939 (Pl. 17, No. 3).

5. Bone buttons. Nine items. Cream-coloured, turned to hemispherical shape. With the exception of one they were sawn into pieces. Dice (?). ${ }^{113}$ Diam: 2.7, H: 0.9 cm. 797-5/1939 (Pl. 17, Nos 5-13).

6. Same as above. Cream-coloured, rounded, scratches on one side. Intact. Diam: 1.9, Th: 0.8 cm. 797-6/1939 (Pl. 17, No. 14).

7. Same as above. Rounded, its top is turned to hemispherical shape. Dark green; with painted yellow circles. Intact. Diam: 2.4, $\mathrm{H}: 0.7 \mathrm{~cm}$. 797-7/1939 (Pl. 17, No. 15).

8. Iron knife. Elongated, leaf-shaped, with one edge. Remains of wood fibre on its tang. L: 15, W: 2.7, Th: $0.9 \mathrm{~cm}$. ("Spearhead" in the A. r.) 797-8/1939 (Pl. 1\%, No. 1).

9. Iron shoe nails. 28 items. With round head. ${ }^{114}$ L: $1.7 \mathrm{~cm}$. 797-9/1939 (Pl. 17, No. 19).

10. Iron fragment. Elongated. L: $4.4 \mathrm{~cm}$. (Maybe the tang of the iron knife under No. 8 above.) 797-11/1939 (Pl. 1\%, No. 1a).

11. Iron fragments. 797-10/1939.

(a) Fragment of ferrule-binding. Flat, bent into a semicircle. L: 1.8 , Th: $0.2 \mathrm{~cm}$.

(b) Iron fragments. Two pieces. Solid, shapeless.

(c) Iron object. Flat, rough surfaced. L: 10.6, W: 5, Th: $0.6 \mathrm{~cm}$.

(d) Same. With one big nail. L: 6.3, W: 4, Th: $0.6 \mathrm{~cm}$. Nail L: $2.3 \mathrm{~cm}$. (A. r. 1910/143).

Graves R/171-176 could not be identified.

\section{1, SZÉKESFEHÉRVÁR STREET}

(Suppl. 2)

Graves R/177-183. In front of the house, below the road, eight Roman tombs were found during canalization works. ${ }^{115}$

113 L. Barkóczi-É. Bónis: Das frührömische Lager und die Wohnsiedlung von Adony (Vetus Salina), Acta Arch. Hung. 4(1954), p. 179, XLV, Nos 7-26; R. M. Alföldi: Intercisa $I I$, p. 486. Cat. No. 2. LXXXIV. No. 6.

114 Burger I, p. 117, Grave 180, Fig. 107; Burger II, p. 69, Grave 23, Pl. 27, 23/5.

115 Gy. Fejes, Dunántúl, 17 April 1927; Gosztonyi I p. 111, Grave LXII. 
Grave R/177. Masonry walls with a roof made of eight pairs of brick $\Lambda$. The bottom is laid with brick. On the top an 8-12 cm thick layer of lime mortar. Inside lined with standing bricks. L: 200, $\mathrm{W}: 50, \mathrm{H}: 40 \mathrm{~cm}$. D: $215 \mathrm{~cm}$. E-W orientation.

A well preserved male skeleton was in the grave (head to the east).

Associated articles:

1. Wooden casket with bronze mounts (beside the head). Joined sides. Th. of wood: $1.8 \mathrm{~cm}$. Bronze mounts around the keyhole and on the surface of the casket; bronze handle. ${ }^{116} 1165 / 1939$ ( $P l .19$, Nos $1-3 ; P l .20$ ).

2. Bronze key with ring-handle. Made of thin bronze wire of $\mathrm{D}$-shape cross section; the ward has three teeth set at a right angle. Diam. of ring: $2.3 \times 3.4$, Th: $0.3 \mathrm{~cm}$. Size of ward: $1.5 \times 0.8 \mathrm{~cm}$. 1106/1939 (Pl. 19, No. 4).

3. Bronze brooch. Three-knobbed, crossbow type brooch. Decorated with four and six punched ringlets on its foot. Intact. L: $9.4 \mathrm{~cm}$. 1148/1939.

4. Glass cup. Light blue with white ribs. Globular, broken.112 DM: 11, H: 5, DB: $3.4 \mathrm{~cm}$. 1209/1939 (Pl. 18, No. 2).

5. Pottery flagon. Dark grey with shiny black surface. On its body vertical trailing tendrilshaped burnishings. Intact. H: 5, DM: 7.3, DB: 8 cm. 800-5/1939 (Pl. 18, No. 1).

6. Coins:
(a) Constantine PB
(2) (C-XII. 30)
(b) Constantine PB
(C-XII. 32)
(c) Constantine PB
(C-XII. 33)

7. Glass vessel. (A. r. 1927/246). NI.

Grave R/178. According to Gy. Fejes ${ }^{118}$ of the eight graves found here "the seven others were found at a distance of one to two metres from one another to the N. Their shape was the same as that of the first one. Not all of them had such a rich quantity of articles." D: $200 \mathrm{~cm}$.

Associated articles:
1. Coin. Probus
Ant. (C-XII. 25)
2. Coin. Probus
Ant. (C-XII. 21)

Grave R/179. Disturbed, without articles. ${ }^{119}$ D: $240 \mathrm{~cm}$.

\footnotetext{
116 On its decoration cf. A. Radnóti, Intercisa $I I, \mathrm{p}$. 260, Figs 53 and 67. LV. Nos 5-6, 8-9.

117 Isings, p. 18, form 3/a; F. Neuburg: Antikes Glas, Darmstadt 1962, Abb. 79; Н. П. Сорокина, Стекло из раскопок Пантикапея 1945-1959 гг. МИА 103 (Москва 1962) 210, Рис. 2. 5. Unknown after the middle of the 2nd century.

118 Gy. Fejes, Dunántúl, 17 April 1927.

${ }^{119}$ Gy. Fejes, ibid.; Gosztonyi I, p. 11. He cannot separate the individual graves: Nos LXIII-LXVII at loc.
} cit.
Grave R/180. Disturbed. ${ }^{120}$ D:160 cm.

Associated articles:

1. Silver buckle: D-shaped, with ornamented, notched tongue. L: 2, W: 1.5 , Th: $0.2 \mathrm{~cm}$. 1107-1/1939 (Pl. 47, No. 7).

2. Fragment of silver plate. Belt-plate with trace of a nail. L: $1.8 \mathrm{~cm}$. 1107-2/1939 (Pl. 47, No. 7).

Grave R/181. D: 165 em. ${ }^{121}$

Associated articles:

1. Fragments of glass scent bottle. Greenish-white, thin-walled. Large size, strongly bulbous type. Its bottom is thick. (Ten fragmentary pieces.) Original L: 45 , L. of largest fragment: $10.5 \mathrm{~cm} .802-1(P l .21$, No. 5).

2. Same as above. Whitish, thin-walled, with spindle-shaped body as the above one (20 fragmentary pieces). Original L: $47, \mathrm{~L}$. of largest fragment: $7.8 \mathrm{~cm} .801-1$.

3. Glass beaker. Broken. H: 7, Diam: 5 and $8 \mathrm{~cm}$. NI.

4. Glass bottle. Yellowish-white, thin-walled. H: (broken) 9, DM: (broken) 2.2, DB: $4 \mathrm{~cm} .1132 / 1939$ (Pl. 21, No. 4).

5. Bottom of glass beaker. NI.

6. Pottery lamp. Its cover-plate is broken; illegible inscription on its bottom. L: $8.3, W: 5.5 \mathrm{~cm}$. 1195/1939.

7. Fragments of pottery lamp. Brownish. L: 4.7 cm. 1196/1939.

8. Pottery vessel. Red with fine glaze, twohandled. H: 15.9, DM: 8.5 , DB: $6 \mathrm{~cm}$. 800-8/1939 (Pl. 22, No. 1).

9. Same. Cream-coloured, with orange paint, two-handled. Intact. H: 13.3, DM: 10.5, DB: $5.5 \mathrm{~cm}$. 800-6/1939 (Pl. 22, No. 3).

10. Pottery flagon. Brick-coloured, dark green glaze outside. H: 16.5, DM: $4, \mathrm{DB}: 4.5 \mathrm{~cm}$. 806-3/1939 (Pl. 22, No. 4).

10a. Pottery vessel. Brick-coloured, coarse. Vase shaped. H: 18.3, DM: 4.9, DB: $5 \mathrm{~cm}$. 801-2/1939.

11. Pottery dish. Dark grey, shiny with worn dark grey paint. H: 3.8, DM: 15, DB: $7.9 \mathrm{~cm}$. 815-13/1939 (Pl. 22, No. 5).

12. Glass bangle. Black with D-shaped cross section, smooth. Diam: $6.6, \mathrm{~W}: 0.7$, Th: $0.5 \mathrm{~cm}$. 1108-2/1939 (Pl. 21, No. 1).

13. Bronze bracelets. Two items.

(a) D-shaped cross section, open, with stylized snake heads. Broken. Diam: 6.1, W: 0.3, Th: $0.2 \mathrm{~cm} .1108-3 / 1939$.

\footnotetext{
${ }^{120}$ Gy. Fejes, ibid.; Gosztonyi I, p. 11.

${ }^{121}$ Gy. Fejes, Dunántúl, 12 April and 17 April 1927; Gosztonyi I, p. 11.
} 
(b) Same, open with square ends, edges rounded. Intact. Diam: 5.6, W: 0.4, Th: $0.3 \mathrm{~cm}$. 1108-3/1939 (Pl. 21, No. 2).

14. Bronze brooch. Pin missing. H: $7 \mathrm{~cm}$. NI.

15. Bronze handle. Flat, formed as two stylized dolphins. Intact, with nice patina. Part of casket(?). L: 8.9, W: 1.4, Th: 0.3 cm. 815-9/1939 (Pl. 21, No. 3).

16. Bone buttons. Six items. NI.

17. Coins:
(a) Diocletian
MB
(C-XII. 26)
(b) Maximian Herc. GB
(C-XII. 27)
(c) Licinian
$\mathrm{MB}$
(C-XII. 28)
(d) Constantine
PB (2) (C-XII. 29)
(e) Constantine
PB (2) (C-XII. 31)
(f) Constantine
PB (2) (C-XII. 34)

Grave R/182. D: $160 \mathrm{~cm} .^{122}$

Associated articles:

1. Glass scent bottle. Light green, spindle-shaped. With a solid, globular base. Very fragmentary. L (original): 43, DM: 2, DB: $1.6 \mathrm{~cm} .1172$.

2. Base of glass jug. White, thin-walled. Narrows conically towards the top, with the fragment of the body starting from a globular moulding. Broken, incomplete. H (incomplete): 4.6, DB: $7 \mathrm{~cm}$. 802-2/1939 (Pl. 22, No. 10).

3. Fragment of the bottom of glass beaker. Diam: $6.2 \mathrm{~cm}$. 1137/1939. E.

4. Fragments of glass jug. Three pieces. NI.

5. Bangles. One of black glass, three of bronze. Two of the latter are complete, one is broken in half. NI.

6. Bronze ring. With pendant. L: $2 \mathrm{~cm}$. NI.

7. Clay lamp. Dark grey lamp. Intact. L: 7.7, H: 2.6, DB: 3.4 cm. 802-3/1939 (Pl. 22, No. 2).

8. Bronze dish. Flat. With a groove around the outer edge of the rim. Patched with metal on the outer side. Diam: 7.1, H: 1.1, Th: $0.3 \mathrm{~cm} .1105 / 1939$ (Pl. 22, No. 11).

9. Pottery beaker. Dark grey, coarse. H: 9, DM: 7.5, DB: $3.7 \mathrm{~cm}$. 802-4/1939 (Pl. 22, No. 6).

10. Fragment of bronze brooch. Crossbow type with three knobs. Only the three knobs and half of its bow remained. L: $2.9, \mathrm{~W}: 4.2$, Th: $0.7 \mathrm{~cm}$. 802-5/1939 ( $P l .22$, No. 7).

11. Bronze buttons. Three items. With small head. 802-10/1939. M.

12. Pieces of bronze wire. Three pieces. (Fragments of brooch ?) L: 4 , Th: 0.3 ; L: $2.7 \mathrm{~cm}$; L: 1.9 , Th: $0.2 \mathrm{~cm}$. 802-8/1939.

13. Pieces of bronze plate. Six pieces. Fragments of brooch and buckle. 802-9/1939. M.

122 Gosztonyi I, ef. footnote 119.
14. Rings of bronze plate. Three items. One of them could be identified: slightly embossed with a hole in the middle. Diam: 2.2 , Th: $0.1, \mathrm{~W}: 0.8 \mathrm{~cm}$. 802-7/1939 (Pl. 22, No. 9).

15. Bronze buckle. Of square cross section, oblong shaped. With movable tongue. L: $3.4, \mathrm{~W}: 2$, Th: 0.4 cm. 802-6/1939 (Pl. 22, No. 8).

Grave R/183. D: $180 \mathrm{~cm} \cdot{ }^{123}$

Associated articles:

1. Pottery vessel. Grey. H: 10, DB: 6, DM: $9 \mathrm{~cm}$. (Pl. 23, No. 2).

2. Base of glass beaker. NI.

3. Bronze brooch. Crossbow type with three knobs. Solid. Six cut pelta ornaments on its foot. Its central knob and pin are missing. L (without knob): 5.9, W: 5.9, Th: 1.1 cm. 803/1939 (Pl. 23, No. 1).

4. Bronze ornamental piece. Clover-shaped with three lobes. Broken. L: 5.8, W: 5.5, Th: $0.3 \mathrm{~cm}$. 815-7/1939. (Identified provisionally from the old and unregistered material.) ( $P l .23$. No. 3).

5. Clay ball. One item. NI.

6. Iron nail. One item. NI.

7. Bronze buckle. NI.

8. Bronze dress ornament (?). Star-shaped. Diam: $3.9 \mathrm{~cm} .1497 / 1939$. E.

9. Coins:

Valentinian I AE 3 (C-XII. 35, 36, 37, 38, 39)

Valens AE 3 (C-XII. 40, 41, 42, 43)

\section{CORNER OF SZÉKESFEHÉRVÁR STREET AND GEISLER ETA STREET}

(Suppl. 2)

Grave R/184. D: 120 cm. ${ }^{124}$ Undisturbed grave, its roof is $\wedge$. Complete skeleton.

Associated articles:

1. Glass scent bottle. Light green, thin-walled. Intact. L: 45.7, DM: $3.1 \mathrm{~cm}$. 801/1 (Pl. 23, No. 8).

${ }^{123}$ Gy. Fejes, Dunántúl, 17 April 1927. He notes for graves 7 and 8 that they were covered with a semi-circular barrel vault. They were built beside each other and the common wall, i. e. the one separating the two tombs, had a hole of 15-25 cm. The W wall was closed by a smoothly carved stone slab; according to Gosztonyi $I$, p. 119, the double vaulted tomb chamber was found at a D. of $1.6 \mathrm{~m}$. According to him there was an opening $15 \times 25 \mathrm{~cm}$ in size in the longer wall which was closed by a carved stone slab. In one of the tombs six coins of Valentinian and one of Valens were found. Because of the identical size of the hole in the separating wall and the identical number of the Valentinian coins we identify this with grave 7 .

124 Notes of Gy. Fejes: JPM-Reference Archives, 218; Gy. Fejes, Dunántúl, 17 April 1927; Gosztonyi I, p. 112, Grave LXVIII. 
2. Pottery flagon. H: 11, DM: 9, DB: $5.5 \mathrm{~cm}$. NI.

3. Bronze buckle. With a D-shaped loop, and plate of triangular shape, with three rivets. L: 4.4, W: 2.9 cm. 1109/1939 (Pl. 23, No. 4).

4. Glass beaker. Greenish-white, thin-walled. Intact. H: 8.5, DM: 5.6, DB: $3.5 \mathrm{~cm} .1111 / 1939$ (Pl. 23, No. 7).

\section{Coins:}
(a) Probus
Ant. (C-XII. 44)
(b) Probus
Ant. (C-XII. 45)

\section{2, SZÉKESFEHÉRVÁR STREET}

(Suppl. 2)

Graves R/185-189. Five Roman graves were found in front of the building during drainage works between 22 and 26 May 1959.125

Grave R/185. Walls were constructed; the two longer walls are of $2-3 \mathrm{~cm}$ thick mud, the two narrower sides are 20-22 cm thick. Made of pieces of stone and brick with lime mortar. Roof is of rimmed bricks: $\Lambda$, with a 14-16 $\mathrm{cm}$ thick layer of mortar on it. SE-NW orientation.

Entirely dislocated, scattered bones.

Associated articles:

1. Fragments of iron knife. Five pieces. With a leaf-shaped blade and short tang with two nails in it. Remains of wood and textile fibres. 62.49.1 (Pl. 18, No. 3).

2. Fragments of glass beaker. (Near the middle of the northern side.) White, thin-walled. Chaliceshaped. H: 7.6, DB: $3.4 \mathrm{~cm}$. 62.49.2.

3. Fragments of bronze earring. Two pieces. Thin wire, with a hook and loop fastening. 62.49.3 ( $P l$. 18, No. 4).

4. Fragments from the side of a pottery vessel. Grey, from a globular vessel. 62.49.4.

Grave R/186. Earth grave, disturbed. It had no articles. SE-NW orientation (head to SE). Bones remained only from about the waist to the feet.

Grave R/187. Earth grave. Only the head and the shoulders were accessible as the grave was below the road. SE-NW orientation (head to SE).

The skeleton is presumably that of an elderly female.

Associated articles:

1. Glass beads (near the neck), 11 items. Blue, green and brown. One is disc-shaped with engraved wavy line on one side; two are in the shape of an

\footnotetext{
125 The excavation of the graves was done by Dr. László Papp.
}

octagonal and quadrangular prism; one is a small green octahedron and one is brown and disc-shaped. 62.50.1 (Pl. 18, No. 5).

Grave R/188. A grave made of brick and limestone with mortar. Its roof is $\Lambda$. Only its south-eastern end was accessible. Entirely dislocated, dispersed human bones.

Grave R/189. Built of brick and limestone. Two pieces of $j$ ts brick roof were lying amid the debris. Entirely disturbed, and around it there were dispersed human bones.

\section{3, SZÉKESFEHÉRVÁR STREET}

In the trench dug for the modernization of the gas pipe-lines, debris was found of graves previously disturbed, and remains of bones were found everywhere at a D. of 150-205 cm.

\section{6, KÁPTALAN STREET}

\section{Fragment of tombstone. ${ }^{126}$}

\section{1, LEONARDO DA VINCI (SZEPESSY) STREET (Suppl. 2)}

In the drainage works of May 1927 the following were found:

1. Fragments of glass beaker. Four pieces. Greenishyellow, thin-walled. Conical, with a slight kick in its base. DB: $2.7 \mathrm{~cm}$. 807/1939.

Grave R/190. In 1927 a disturbed Roman grave was found at the same place, in front of the school. ${ }^{127}$ D: $200 \mathrm{~cm}$. Made of brick $(60 \times 60 \times 7 \mathrm{~cm})$. The skeleton was covered with red sand.

Associated articles:

1. Gilt bronze brooch. Crossbow type with three knobs. Its bow is smooth and plain. Its foot is ornamented at both sides with six cut in peltamotifs. It has worn traces of gilding on its shoulder and bow, the pin is missing and the knobs are damaged. It is light and hollow inside. L: 8, W: 5.1 cm. ${ }^{128}$ 1117/1939 (Pl. 23. No. 6).

\section{Bronze end of chain. NI.}

126 Stone Finds, Cat. No. 28.

${ }^{127}$ Gy. Fejes, Dunántúl, 17 April 1927; Gosztonyi $I$, p. 112, Grave LXXIII.

128 Patek, p. 266, 18a, No. 58 (our copy); Burger I, grave 198, p. 120, note 240, Fig. 109; Keller, p. 41 and supra, typ. 5 . 
3. Bronze pendant. Crescent-shaped with a ring at the middle. One end broken. L: $4.5, \mathrm{H}: 1.6$, Th: 0.4 cm. 815-5/1939 ( $P l$. 23, No. 5).

4. Pottery flagon. Grey. With pouring lip and ribbon handle. Intact. $\mathrm{H}$ : 18.8, DM: 8.3 , DB: $6.7 \mathrm{~cm}$. 800-3/1939 (Pl. 23, No. 9).

5. Fragments of flagon (?). Two pieces. Glazed. (A. r. 1927/340-344). NI.

\section{SZECCHENYI SQUARE (THE FORMER CSÉBY BUILDING) (Suppl. 2)}

In 1897, when the headquarters of the Reserve Bank were built on Széchenyi Square, the Cséby building was pulled down and three Roman graves were found. ${ }^{129}$

Grave $\mathbf{R} / 191 .^{130}$ According to Várady it was a brick grave and its top was covered by stone slabs. ${ }^{131}$ Size and depth are unknown.

Associated articles:

1. Glass jug. Yellowish-green, thick-walled. Long, cylindrical body, below its double, outfolded rim is a broad ribbon handle bent at a right angle. The body of the vessel is divided into five fields by four cut horizontal lines; three of the fields are broader, two are narrower. ${ }^{132} \mathrm{H}: 29.5$, DM: 10.5, DB: 13 cm. HNM 108/1912.3 (Pl. 24, No. 1).

2. A pair of gold earrings. ${ }^{133}$

3. Gold necklace. ${ }^{134}$

4. Gold finger ring. Strong, notched gold wire. A small, plain, oval garnet in a framed setting. Worn. Diam: 1.8, Th. of wire: 0.15 , L. of head: $0.8 \mathrm{~cm}$. Weight: 2.29 g. HNM 108/1912.26 (Pl. 3\%, No. 5).
5. Coin: Trajan GB
6. Sacrificial bowl (?). NI.
(C-XII. 47).

${ }^{129}$ Arch. Ért. (1897) p. 287; Baranya II, p. 196; O. Szőnyi, PBMÉ (1914) p. 91; Gosztonyi I, p. 107.

${ }^{130}$ Gosztonyi I, p. 107, Grave XXV.

131 Baranya II, p. 196.

132 Benkö, p. 107, 4/h.24. No. XVIII (in our copy); Doppelfeld I, pp. 12-13. Abb. 2. From the sarcophagus of Cologne-Braunsfeld, where it was found together with the early Christian "vas diatretum" of Cologne with Greek inscription; Doppelfeld II, p. 64, Abb. 150; a similar one can be found in Munich: Prähistorische Staatssammlung. Inv. No.: 1965/796. With a note "originating from Asia Minor", but the handle is different. Information kindly given by Mr. H. J. Kellner (Munich), for which I would like to thank him here.

${ }^{133}$ See in the material of the HNM Nos: 108/1912. $10-11 ; 22-23 ; 24-25$. Of the three our specimen can no longer be identified.

${ }^{134}$ See in the material of the HNM under Nos 108/1912. $9,16-17$. We can no longer identify it.
Grave R/192. ${ }^{135}$ Presumably of similar construction to the previous one. Size and depth are unknown.

A. Horváth deals with the above graves, found during the construction of the Reserve Bank in 1897, in his letter dated 26 April 1897.136 The contents of the letter contradict the above description by B. Posta, ${ }^{137}$ insofar as he states that in one of the graves a "glass vessel of amphora shape" was found, and in the other one a "beaker without a stand". The beaker is identical with the cut one with a Greek inscription, which is now in the HNM. ${ }^{138}$

1. Glass beaker. Dark green, thick-walled. Its rim is cut, the edge is slightly thickened. Below the rim engraved inscription runs around in a 1.8 cm wide field ( $P l$. 25). The body of the beaker was ornamented with deep cuts below the inscription. ${ }^{139} \mathrm{H}$ : 15.2, DM: 12.8, DB: $3.5 \mathrm{~cm}$. HNM 108/1912.4 (Pl. 24, No. 2; $P l$. 25).

2. Gold ring. NI.

3. Bronze bracelet. NI.

4. Coin. NI.

Grave R/193. ${ }^{140}$ The structure of this grave was presumably similar to the above ones, but its dimensions and depth are unknown. Disturbed, it was found empty.

In his letter A. Horváth also refers to the bronze vessels found in the graves. The object indicated for Grave R/191 as "sacrificial vessel" may be one of these. He puts the bronze vessels in his letter into the plural. From the collection of A. Horváth two bronze vessels were handed over to the Pécs Museum: we tentatively identify these two bronze vessels with the ones mentioned in the letter.

1a. Bronze jug. Smooth, thin-walled. Its rim is inturned, and forms a horizontal edge. Fragmented, its bottom is missing. The site of one handle may be identified on its side. ${ }^{141} \mathrm{H}: 16, \mathrm{DM}: 6.8$, DB: $6.2 \mathrm{~cm}$. 745/1939 (Pl. 49, No. 2).

\footnotetext{
135 Gosztonyi I, p. 107, Grave XXVI.

136 From the bequest of István Paulovics: HNM Reference Archives, 63. P. II.

137 Baranya $I I$, p. 196.

138 HNM 108/1912.4; in the communication published in Arch. Ért. (1897) p. 267 an amphora-shaped glass vessel and a "beaker without stand" with an incised Greek inscription are mentioned.

139 O. Szőnyi, $P B M E ́$ (1912) p. 163; O. Szőnyi, $M K E ́$ VII (1913) p. 66; Nagy, p. 39, Fig. 8, Note 3 for further literature; O. Doppelfeld: Kölner Jb. Bd. 5(1960-61) p. 24; Doppelfeld I, p. 417 and supra; Burger $I$ p. 121, note 254 for further literature, Fig. 100.

140 Gosztonyi I, p. 107, Grave XXVII.

141 Radnóti I, XLII, No. 8.
} 
1b. Bronze jug. With inturned rim. Turned, with a thicker bottom plate. The base of its cast handle is ornamented with a Medusa head among tendrils. ${ }^{142} \mathrm{H}: 21.8$, DM: 7.2, DB: $7.2 \mathrm{~cm} .746 / 1939$ (Pl. 49, Nos 1 and 1a).

In the collection of A. Horváth, glass beads are listed under serial No. 31 as from the site of the Reserve Bank of Pécs (A. r. 1912/187). In the Inventory of the Pécs Museum (under No. 791/1939):

2. Beads. One flat item, shaped like a melon seed, of black glass paste; one has three segments and is elongated, in dark blue; two big round ones are of white glass; three items are dark grey and conical; three are large, rounded, conical, with green, yellow and brown paint; eight items are of light blue glass paste, flat and rounded; twelve dark greenish-blue ones are small, rounded, of glass paste; nine are yellow, flat and rounded. 791-1,2/1939 (Pl. 26, Nos 5-6).

\section{SZECHENYI SQUARE (THE TOWN HALL BUILDING)}

In 1905, during the reconstruction of the Town Hall building of Pécs two fragments of tombstones were found on the site. ${ }^{143}$ (A. r. 1905/179). NI.

In 1906, during the construction works 11 different objects of the Roman period were found (A. r. 1906/117). NI.

In 1909 from the site of the Town Hall building a Roman lamp was sent to the Pécs Museum with a stamp SIC on its bottom. 1887. NI.

\section{SZECHENYI SQUARE}

During the drainage works in 1926 a coin of Hadrian was found ${ }^{144}$ (A. r. 1929/191.7). NI.

\section{CORNER OF SZÉCHENYI SQUARE AND HUNYADI JÁNOS (INCZÉDY DÉNES) STREET (Suppl. 2)}

Graves R/194-195. In 1927, during the drainage works $9.5 \mathrm{~m} \mathrm{~S}$ of 13 , Széchenyi Square, and $3.5 \mathrm{~m}$ $\mathrm{E}$ of the corner of Inczédy Dénes Street, a sarco-

142 Radnóti I, XLII, No. 5.

${ }^{143}$ Gosztonyi I, p. 123, Nos 31-32.

144 Dunántúl, 19 September 1926.

145 Notes of Gy. Fejes: JPM-Reference Archives, 221; Gy. Fejes, Dunántúl, 13 January 1927; 16 January; (Interview with O. Szőnyi): 1 March and 17 April; Nagy, p. 40; Gosztonyi I, p. 118; Gosztonyi IV, p. 32. phagus made of stone and brick covered with a barrel vault was found. D: $225 \mathrm{~cm}$. Orientation: E-W. ${ }^{145}$ (Burial-chamber XII?)

Grave R/194. L: 195, W: 72, H: $80 \mathrm{~cm}$. The inside of the sarcophagus was plastered, and in addition a painting was found on it. The walling of the western side was presumably done after the inhumation, as this wall was not covered with plaster and was not painted from the inside like the other three. The sides and the barrel vault of the sarcophagus were ornamented with geometric stripes. The painting of the walls consisted of a band of dark blue, a $5.5 \mathrm{~cm}$ wide band of red, and a yellow band $6 \mathrm{~cm}$ wide. At the bottom the whole was framed by a black line. On the western end of the northern and southern walls, below the stripes, patches were identified which may be regarded as representations of animals with bent body. ${ }^{146} \mathrm{On}$ the fragmented vault there were also some patches of painting without any definite design. The barrel vault of the sarcophagus was found in a dilapidated condition and the tomb was filled with soft earth. Its bottom was of smoothed earth. In the tomb there were arm and leg bones, glass fragments and bronze plates. Human bones were found outside the tomb as well. The sarcophagus was carried to the Pécs Museum. ${ }^{147}$ 1279/1939.

Grave R/195. A second sarcophagus, identical with the one described above, was attached to it. ${ }^{148}$ Its two longer sides and its eastern end were found to be $42 \mathrm{~cm}$ in length. It was built of brick. This also had a barrel vault but it had already been demolished. The $\mathrm{N}$ wall of the sarcophagus was attached to the $\mathrm{S}$ wall of the previous one. No traces of plaster could be found on its walls. In the disturbed tomb a skull and a few broken bones were found.

\section{5, HUNYADI JÁNOS STREET (Suppl. 2)}

Grave R/196. This was found in 1927 , in front of the house, during the drainage works. Built of limestone, it is a carved sarcophagus of a child. D: $200 \mathrm{~cm}^{149}$

146 Gosztonyi IV, p. 32 clearly speaks about figurative painting with animals.

${ }^{147}$ Gosztonyi I, p. 118; Gosztonyi IV, p. 32 for further bibliography, Pl. 4.4.

148 Gy. Fejes, Dunántúl, 17 April 1927; Gosztonyi I, p. 118; Gosztonyi IV, p. 34.

149 Stone Finds, Cat. No. 61. 


\section{3, SZÉCHENYI SQUARE}

In 1910, during the construction of the cellar of the house, the following were found:

1. Bronze object with handle, a square shaped handle with a round hole in the middle. Below it there is a small cross-hatched bronze bar. Diam: 3.8, H: $2.3 \mathrm{~cm} .2245$ ( $P l .47$, No. 5).

2. Coins: 69 items. 2056/1910. NI.

\section{2, SZÉCHENYI SQUARE}

Graves R/197-198. In 1963 while laying the foundations of the house two unfurnished graves were found. $.^{150} \mathrm{~W}-\mathbf{E}$ orientation.

\section{SZECHENYI SQUARE}

Graves R/199-200. In 1930, in front of Hotel Nádor, on Széchenyi Square, two disturbed Roman graves and eleven coins were found (Constantine I, Constantine II and Valens).

Gy. Fejes ${ }^{151}$ mentions Roman bricks, glazed fragments of vessels, fragments of a pottery flagon, iron nails and walls.

\section{SZÉCHENYI SQUARE}

Without further data:

\section{Coins:}

(a) Aurelian

(b) Probus

(c) Licinius

(d) Valentinian I.

Ant. (C-XII. 48) M.

Ant. (C-XII. 49) M.

Ant. (C-XII. 50)

AE 3 1366. NI.

\section{SZECHENYI SQUARE, WESTERN SIDE}

(Suppl. 2)

Grave R/201 (?). In 1959, when an electricity pole was installed at the SE corner of the Nagy Lajos Grammar School, the following were found:

1. Fragment of cornice brick. Red, baked clay. Coarsely moulded head of a ram with open mouth, with straight lines scratched on the two sides. With a red painted stripe on both sides. ${ }^{152}$ L: 12, $\mathrm{W}: 7.5, \mathrm{H}: 8.9 \mathrm{~cm}$. 59.222 .1 ( $P l .47$, No. 2).

150 Report of A. Kiss: HNM Reference Archives VII. 195/1963.

${ }^{151}$ Gy. Fejes, Dunántúl, 25 December 1930; Idem PBMÉ (1930) p. 98.

152 Cf. footnote 64.
2. Fragment of painted stone wall. Of yellowish sandstone; in the intact surface traces of curvilinear grey and black painting in two zones. W: $9.2 \mathrm{~cm}$. 59.222.2.

\section{SZÉCHENYI SQUARE CITY PARISH CHURCH}

In 1894 P. Gerecze ${ }^{153}$ mentioned two Roman heads enclosed in the fabric of the wall of the building behind the City Church.

\section{SZÉCHENYI SQUARE CITY PARISH CHURCH (Suppl. 2)}

Graves R/202-217. In 1939, during the demolition and reconstruction of the church, graves of a section of a late Roman cemetery were found. Excavations by Gy. Török. ${ }^{154}$

We now re-process this section of the cemetery, consisting of 16 graves. This is necessary because in the interval since the excavations some objects had been lost, others could be better identified and the coins could be more precisely dated. ${ }^{155}$ Here a brief survey of the graves is given on the basis of the newly published map of the cemetery ( $P l .27)$, the drawings presenting the objects by graves and by the coins arranged in chronological order in the plates (C-XIII).

Gy. Török mentioned a Roman building of $7.66 \times 6.58 \mathrm{~m}$ and two stones with inscriptions in addition to the graves. He relates the Roman building to the cremation burials customary in the early period. In the building there were melted pieces of bronze, fragments of bones, an iron fitting, and fragments of pierced bronze vessels in the ashes. 2026-2032.

Grave R/202. Of brick and limestone, its roof: $\Lambda$. D: 290, L: 196, W: $48 \mathrm{~cm}$. Orientation: $335-175^{\circ}$ $\mathrm{N}-\mathrm{S}$. Disturbed; the orientation of the skeleton is unknown.

At $1.5 \mathrm{~m}$ from the grave the following was found:

1. Clay lamp. L: 7.7, H: 2.5 om. 1577. (Pl. 28). M.

153 P. Gerecze, Arch. Ért. (1895) p. 75.

${ }^{154}$ Török I, p. 125 and supra; Gy. Török, PMÉ (1941) p. 5 .

$155 \mathrm{Cf}$. our plate of coins C-XIII, where the newly processed coin material is published in chronological order on the basis of the definitions of $R I C$ and $L R B C$. 
Grave R/203. Earth grave, its roof: $\wedge$. D: 290 , W: $75 \mathrm{~cm}$. Orientation: $350-170^{\circ} \mathrm{S}-\mathrm{N}$ (head to S). A child's skeleton.

Associated articles:

1. Pottery flagon. Brick-red, with lip. Outer surface covered with brownish-yellow glaze. (Found at the outer side of the left foot.) H: 12.8, DM: 3.2, DB: $3.5 \mathrm{~cm} .1578$ (Pl. 28, No. 3).

2. Pottery bowl (at the outer side of the left foot). Blackish-grey, coarse, thick-walled. Inside there were chicken bones. H: 5.2, DM: 17.8, DB: $7 \mathrm{~cm}$. 1579 (Pl. 28, No. 2).

3. Glass beaker (at the outer side of the left foot). Greenish, thin-walled. With a groove. H: 5.4, DM: 8.8, DB: $2.5 \mathrm{~cm} .1580$ (Pl. 28. No. 1).

Grave R/204. Brick grave, disturbed. 329-149 ${ }^{\circ}$. D: $280 \mathrm{~cm}$. The orientation of the skeleton is unknown.

Associated articles:

1. Glass jug. Bluish-green, thin-walled. Applied trail ornament around its neck; its handle is ribbed. Entirely broken. Original H: 21, DM: 5.5, DB: $5.3 \mathrm{~cm} .1581$ (Pl. 28, No. 1).

2. Glass beaker. Of conical shape. "It may have been $13 \mathrm{~cm}$ high." 1582 (Pl. 28, No. 2). E.

3. Coins:
(a) Constantius II
(C-XIII. 13)
(b) Constantius Gallus $\mathrm{PB}$
(C-XIII. 17)
(c) Jovian
PB
(C-XIII. 18)

Grave R/205. Made of brick and limestone, its roof: $\wedge$. Disturbed. D: 436, L: 168, W: $45 \mathrm{~cm}$. Orientation: $\mathrm{E}-\mathrm{W}, 80-260^{\circ}$. The skeleton is extended (head to $\mathrm{E}$ ).

Associated article:

1. Iron nail. Of square cross section (it was found at the feet). L: $10, W: 0.9$, Th: $0.8 \mathrm{~cm}$. L. of head: $2.2 \mathrm{~cm} .1586$ ( $P l .28)$.

Grave R/206. Entirely demolished.

Associated article:

1. Glass bottle. Light bluish-green, thin-walled. Indented on all four sides. Entirely broken. DB: $4.3 \mathrm{~cm} .1587$ (Pl. 28).

Grave R/207. Entirely demolished. May have been a double grave (?). A male skeleton inside.

Associated articles:

1. Iron knife. With single edged blade of leaf shape. L: 16 (handle: $5.9 \mathrm{~cm}$ ), W: $3.7 \mathrm{~cm} .1588$ (Pl. 29, No. 8). M.

2. Fragments of iron knife. Leaf-shaped, with single-edged blade. L: 9.8, W: 2.7 , Th: $0.5 \mathrm{~cm}$. 1589 (Pl. 29, No. 7).

3. Glass jug. Yellowish-green, thin-walled. With a fluted globular body. Entirely broken. H: -, DM: 5.2, DB: $4.5 \mathrm{~cm}$. W: of handle: $0.9 \mathrm{~cm} .1590$ (Pl. 29, No. 1).

4. Bronze brooch. Crossbow type, three-knobbed. Its body is pierced by two holes and ornamented with rows of garlands. With safety device. Pin missing. L: 6, W: $5.6 \mathrm{~cm} .1591$ (Pl. 29, No. 6).

5. Fragment of bronze brooch burnt to "shapelessness". L: 3.3, W: $3.3 \mathrm{~cm} .1592$ (Pl. 29, No. 4).

6. Bronze buckle. Square-shaped loop with triangular cross section, and movable tongue. L: 3.9, W:2.5, Th: $0.3 \mathrm{~cm} .1593$ (Pl. 29, No. 3).

7. Bronze strap tag. Amphora-shaped, with two rivets on top and two pierced handles of pelta shape. With a drop-shaped ornament at the bottom. L: 5.4, W: 2.9, Th: $0.2 \mathrm{~cm} .1594$ (Pl. 29, No. 5).

8. Bronze buckle. With a loop of triangular cross section. Oval shape. With movable tongue. Two rivets in the plate. L: $4.3, \mathrm{~W}: 3.9$, Th: $0.4 \mathrm{~cm}$. 1595 (Pl. 29, No. 2).

9. Coins: 13 items.
(a) Jovian
PB (C-XIII. 19)
(b) Valentinian
AE 3 (C-XIII. 20-30)
(c) Gratian
AE 3 (C-XIII. 31)

Grave $\mathbf{R} / \mathbf{2 0 8}$. Completely ruined.

Associated article:

1. Pottery beaker. Grey, finely precipitated. With a flat, divided strap handle. H: 10.5, DM: 9, DB: $5.4 \mathrm{~cm} .1615$ (Pl. 29).

Grave R/209. Completely ruined. On the basis of the associated articles the skeleton was apparently female.

Associated articles:

1. Base of a flagon. Black-grey, of clay containing mica. DB: $3.9 \mathrm{~cm} .1616$ ( $P l .29$, No. 6).

2. Bronze button. Hemispherical shape with a hole in the middle. Diam: 2.5, H: 1.1, Th: $0.5 \mathrm{~cm}$. 1617 (Pl. 29, No. 2).

3. Bronze ring. Of thin, notched wire. Penannular, at one end is a stylized snake head. Diam: 2.2, W: 0.6, Th: $0.2 \mathrm{~cm} .1618$ (Pl. 29, No. 3).

\section{Fragments of bracelets:}

(a) A flat bronze strip with engraved decoration. Open. W: 0.5, Th: $0.3 \mathrm{~cm}$. Diam: about $7 \mathrm{~cm} .1619$ (Pl. 29, No. 1).

(b) A flat, tapering bronze wire with twisted ends. Diam: $7.6 \mathrm{~cm} .1619$ (Pl. 29, No. 4).

5. Glass bangle, Black glass. Incomplete. Diam: 7, Th: $1 \mathrm{~cm} .1620$ (Pl. 29, No. 5).

Grave $\mathbf{R} / \mathbf{2 1 0}$. Built of stone, its top is of rimmed brick $\Lambda$, its bottom is earth. Orientation: $\mathrm{S}-\mathrm{N}$, 10-190 ${ }^{\circ}$. A child's skeleton (head to the S). 
Associated articles:

1. Pottery beaker (at the feet). Grey with one handle. H: 10.2, DB: 4.4, DM: $8.2 \mathrm{~cm} .1622$ (Pl. 29, No. 4). M.

2. Iron knife (in the same position). Leaf-shaped, with a long tang. L: 13.5, W: $13.6 \mathrm{~cm} .1623$ (Pl. 29, No. 2).

3. Fragment of bronze bracelet (in the same position). Of cylindrical wire, open. Ends in snake heads, broken. Diam: $3.8 \mathrm{~cm} .1624$ ( $P l$. 29, No. 3).

4. Bezel of ring (in the same position). With convex decoration. Diam: 1.3, Th: $0.4 \mathrm{~cm} .1625$ (Pl. 29, No. 1). M.

Grave R/211. This could not be excavated. Near the grave the following was found:

Pottery beaker. Orange-red, thin-walled. Finely granulated. With bands of impressed markings, and between them three stars with 12 spokes each made by the barbotine technique, with orange-red paint. H: 13.3, DM: 8, DB: $4.1 \mathrm{~cm}$. 1626 (Pl. 30).

Grave R/212. Earth grave. D: 180, L: 165, W: $60 \mathrm{~cm}$. Orientation: E-W. 100-280'.

There was a male skeleton (head to the E). Extended, with arms bent at the elbows.

Associated articles:

1. Glass beaker (at the outer side of the left foot). Greenish-white, thin-walled. Broken. H: 11.2, DM: 8, DB: $4.5 \mathrm{~cm} .1627$ (Pl. 30, No. 2).

2. Bronze brooch (on the right collar-bone). Three-knobbed, crossbow type. Twisted wires below the knobs. Intact. L: 9.5 , W: $5.9 \mathrm{~cm} .1628$ (Pl. 30, No. 1; Pl. 32, No. 2).

3. Iron knife (below the right elbow). Large, leaf-shaped. With a long tang, which ends in an oval bronze stud. Broken, complete L: 24.5, L. of blade: 13, W: 5.5, Th: $0.5 \mathrm{~cm} .1629$ (Pl. 30, No. 3).

4. Coins: (in the left hand) 12 items.
(a) Urbs Roma
PB (3) (C-XIII. 1-3)
(b) Constantine I
PB (C-XIII. 4)
(c) Constantine II
PB (2) (C-XIII. 5-7)
(d) Constans
PB (C-XIII. 8)
(e) Constantius II
$\mathrm{PB}(5)$
(C-XIII. 9-12,

14-15)

5. Iron object (beside the right thigh-bone). Flat, it is $12 \mathrm{~mm}$ wide and $3 \mathrm{~mm}$ thick at its edge. The other end is missing. Chisel (?). L: 8 and $16 \mathrm{~cm}$. 1644 (Pl. 30, Nos 4a-b).

6. Fittings of bronze belt:

(a) Bronze buckle. The loop is formed of two dolphins holding a shell in their mouths. Their heads are pierced at the mouth and the shell is pierced in the middle. It has an ornamented tongue.
The belt plate is oval, with three rivets. ${ }^{156} \mathrm{~L}: 4.6$, $\mathrm{W}: 3.7 \mathrm{~cm}$. (Below the right elbow; additionally one rounded and two "propeller variants" were found.) 1648 (Pl. 30, No. 5; Pl. 32, No. 1).

(b) "Propeller"-shaped fittings. Nine items. Their central, rounded part consists of a disc with two concentric grooves. There is a hole in the middle. The smallest one is badly worn. ${ }^{157} \mathrm{~L}$. of one item: 2.9 , of six items: 3.3 , of two items: $3.5 \mathrm{~cm}$. 1649 (Pl. 30, No. 7; Pl. 32, No. 1).

(c) Round repoussé plate. Four items. With masks representing an embossed head of Medusa inside a beaded border. Fragmentary, broken. ${ }^{158}$ Diam: 3, Th: $0.1 \mathrm{~cm} .1650$ (Pl. 30, No. 6; Pl. 32, No. 3).

(d) Bronze rivets. Two items. They give some indication of the thickness of the leather belt. ${ }^{159}$ L: 8 mm. 1646 (Pl. 30, No. 8; Pl. 32, No. 4).

Grave $\mathbf{R} / 213$. This could not be excavated, The following was found at the edge of the grave:

Pottery bowl. Brick-red, with worn yellow glaze inside. Intact. H: 4.4, DM: 14.2, DB: $6.4 \mathrm{~cm} .1651$ (Pl. 31).

Grave R/214. Brick grave (?). D: 190, L: 150, $\mathrm{W}: 50 \mathrm{~cm}$. Skeleton of a child (boy?) (head to the E). Associated articles:

1. Glass beaker (between the two feet). White, totally broken. Original H: 11.6, DM: 8.2 , DB: $3.4 \mathrm{~cm} .1652$ (Pl. 31, No. 2).

2. Glass cup (between the two feet). It was in the shape of a half egg, but it is totally broken. DM: 8-10 cm (Pl. 31, No. 3). M.

3. Pottery bowl. (By the occipital bone.) Corresponds to the bowl of Grave R/203 (1759). There were chicken bones in it. H: 5.2, DM: $17.8, \mathrm{DB}$ : 7 cm. 1653 (Pl. 31, No. 5). M.

4. Bronze belt fitting (on the left pelvis). Heartshaped ending, a pair of stylized animal heads above, with two rivets representing their eyes. Broken. L: 3.4, W: 2.2, Th: 0.1 cm. 1654 (Pl. 31, No. 4).

156 J. Dombay, Későrómai temetők Baranyában (Late Roman Cementeries in Baranya), JPMÉ (1957) p. 269 for further bibliography; L. Barkóczi, New data on the history of late Roman Brigetio. Acta Ant. 13(1965) p. 231, No. 7, Pl. XXI, 4. (grave 4); Martin, p. 1 and supra; cf. the buckle from Grave L/96 of the cemetery of István Square, Fülep $V$.

${ }^{157}$ J. Dombay, ibid.; Burger I, p. 56, Graves 8-13, 105, Fig. 98; Martin, p. 17, Note 3; Keller p. 67 (propellerförmig) for further bibliography.

${ }^{158}$ A. Radnóti, Intercisa II, p. 339, Cat. No. 78, Fig. 65. Pl. LXIV, No. 2, 4; p. 342, Cat. No. 86, Fig. 83; Intercisa $I I$, p. 91 , Note 29 . He attributes an apotropaic role to the Medusa-head on the scrinia.

${ }^{159}$ Keller, Taf. $35 / 6$. 


\section{Coins:}

Constantius II PB (2) (C-XIII. 12, 16).

6. Bronze brooch (on the right collar-bone). Three-knobbed, crossbow type. A groove runs along its bow (reconstructed on the basis of a photograph). W: 3.4, L: 5.5 cm. 1657 (Pl. 31, No. 1).

Grave R/215. Brick grave, ruined. D: $210, \mathrm{~W}$ : $62 \mathrm{~cm}$. Orientation: E-W, head to the E. A male skeleton inside.

Associated article:

Bronze brooch. Three-knobbed, crossbow type. Five pairs of pelta ornaments on the foot. Intact, but the pin is missing. L: $9.1, \mathrm{~W}: 5.3 \mathrm{~cm} .1658$ (Pl. 31).

Grave R/216. Completely demolished.

Associated article:

Pottery beaker. Grey, of coarse, granulated clay. With a ribbed handle. H: 9.7, DM: 7.4, DB: $3.5 \mathrm{~cm}$. 1660 (Pl. 31).

Grave R/217. This could not be excavated.

\section{JÓKAI (KIS) SQUARE (Suppl. 2)}

Grave R/218. Gy. Gosztonyi, referring to O. Szönyi, mentions three Roman graves in the western part of Jókai Square. ${ }^{160}$

\section{3, JOKKAI SQUARE}

Above the inner arch of the gateway there is a fragment of an inscription.

Fragment of tombstone. ${ }^{161}$

\section{3, SALLAI (FERENCIEK) STREET}

A fragment of a tombstone with relief is built into the wall of the house below the gateway.

Fragment of a tombstone. ${ }^{162}$

\section{9, SALLAI STREET}

In the cellar of the house:

Fragment of tombstone. ${ }^{163}$

${ }^{160}$ Szönyi I, p. 9, Pl. 1; Gosztonyi $I$, p. 108, Grave XXX.

161 Stone Finds, Cat. No. 33.

162 Stone Finds, Cat. No. 19.

${ }^{163}$ Stone Finds, Cat. No. 47.

\section{0, SALLAI STREET}

The following was found in 1926 during drainage works:

Bronze coin of Domitian (A. r. 1926/181/7).

\section{0, SALLAI STREET}

One coin was found when the foundations of the house opposite the church were built:

Bronze coin. NI. (A. r. 1035/a/1941).

\section{SALLAI STREET, PÓSZ BUILDING}

(Suppl. 2)

Graves R/219-220. Two brick graves were found in the courtyard of the building in 1886. D: 253 $\mathrm{cm} .^{164}$ The side walls were built of rimmed bricks and the roof is also of bricks. Orientation: E-W. There were skeletons in the graves.

Associated articles:

1. Coins of Antoninus Pius. NI.

2. Bronze vessel. NI.

3. Necklace of beads. NI.

4. Pottery vessels NI.

\section{SALLAI STREET}

Built into the walls of various buildings:

1. Fragment of tombstone. ${ }^{165}$

2. Fragment of tombstone. ${ }^{166}$

3. Fragment of tombstone. ${ }^{167}$

\section{VÁRADI ANTAL STREET}

Exact site unknown:

Fragment of tombstone. ${ }^{168}$

\section{1, VÁRAdI ANTAL STREET (Suppl. 2)}

Grave $\mathbf{R} / 221$ (?). During the drainage works in front of the house Roman bricks and debris were found at a D. of $350 \mathrm{~cm}$ (A. r. 1927.184/50).

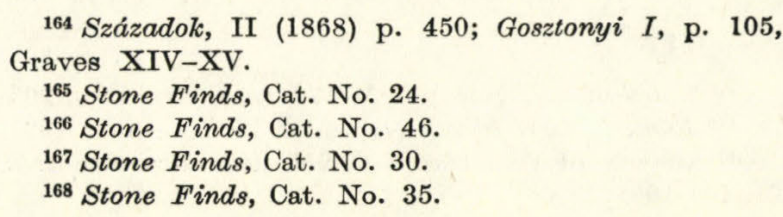


7/2, VÁRADI ANTAL STREET (Suppl. 2)

Grave R/222 (?). During drainage works, at a D. of $300 \mathrm{~cm}$ the following was found:

Pottery beaker. Light brown, finely granulated. Diagonal lines in several rows on its side. Complete. H: 9.5, DM: 7.5, DB: 4.8 cm. 810/1939 (Pl. 26, No. 3).

\section{6, BEM JÓZSEF (IRGALMAS) STREET} (Suppl. 2)

Grave R/223. This was found in 1927, during drainage works, D: $145 \mathrm{~cm}$.

Associated articles:

1. Iron ring. NI.

2. Iron nails. Two items. With flat head. L: 6.6 and $6.4 \mathrm{~cm} .1112 / 1939$ ( $P l .26$, Nos 7-8).

3. Bronze plate. NI.

4. Coin. Constantius II AE 3 (C-XII. 51).

5. Coin. Bronze coin. NI. (A. r. 1927/350-351).

\section{7, RÁKóCZI ROAD}

In 1927, during drainage works, pieces of brick and coins were found:169

Coins:

(a) Constantinus Aug. NI.

(b) Providentiae Aug. NI.

\section{9, RÁKóCZI ROAD (Suppl. 2)}

Grave R/224. On 5 December 1927, at a D. of $225 \mathrm{~cm}$, bricks and bones were found during drainage works, and included the following finds: ${ }^{170}$

1. Bone knife. NI.

2. Coin: Maximinus Daza GB (?) with the inscription: GAL. VAL. MAXIMINV NOB C and GENIO CASARIS (sic!); NI.

3. Coins: Two items. Constantine I (IMP CONSTANTINVS PF AVG and SOLI INVICTO COMITI); NI.

\section{9/c-d, RÁKóCZI ROAD}

According to the description of Gy. Török ${ }^{171}$ the scattered human ashes between the D. of 110 and

\footnotetext{
169 Notes of Gy. Fejes: JPM-Reference Archives, 205. 170 Ibid., p. 204; Idem., Dunántúl, 25 December 1927. 171 Report of Gy. Török: HNM Reference Archives, V. $104 / 1968$.
}

$200 \mathrm{~cm}$ indicate cremation burials. Traces of cremation burials and the bronze vessels represent an earlier period. (A. r. 1930.922.1-122.)

\section{9, RÁKóCZI ROAD (Suppl. 2)}

Gy. Török ${ }^{172}$ found a male skeleton, head to the $\mathrm{W}$, feet to the $\mathbf{E}$, below the road surface, at a $\mathbf{D}$. of $325 \mathrm{~cm}$ :

Grave R/224/a. Disturbed.

\section{8, RÁKóCZI ROAD (Suppl. 2)}

In 1955 the author conducted excavations in Hal Square and Rákóczi Road. ${ }^{173}$ In trench 2 there was a thick layer of debris below the medieval road surface, with characteristic remains of burning. There was apparently a cremation ground (ustrina?) here, and there are remains of charcoal, ${ }^{174}$ and burnt human bones. Two cremation burials were found:

Grave R/225. Its sides were built of brick laid edgewise, outer L: 159, inner L: 137, W: $32 \mathrm{~cm}$. The eastern side was badly damaged. Fragments of human bones were found above the grave as well. Disturbed.

Associated articles:

1. Buckle tongue. Bronze, twisted; L: $2.1 \mathrm{~cm}$. 55.34.6 ( $P l$. 33, No. 1).

2. Fragment of vessel. Grey, a small base sherd. 55.34.2.

3. Fragment of vessel. Dark grey, coarse, sooted on the outside. 55.34.3.

4. Animal bones. One canine tooth, one fragment of shin-bone. ${ }^{175} 55.34 .5$ ( $P l$. 33, No. 2).

In the NW corner of the trench there is a burnt area, thickness about $15 \mathrm{~cm}$.

Grave $\mathbf{R} / 226$. This grave was built of stone and pieces of brick along the eastern side of the trench.

172 Ibid., V. 116/1968.

173 Its processing cf. Frülep $V$.

174 Identified on the basis of the test of Dr. József Stieber (Reader in the Department of Applied Botany, Faculty of Sciences, Eötvös Lóránd University) as: "Qr (Quercus of robur = robur) 10."

175 According to the definition of Sándor Bökönyi they are pig bones. - Pig bone in cremation burial, cf: M. v. Chlingensperg auf Berg: Die römischen Brandgräber bei Reichenhall, Braunschweig 1896, p. 10. 
Orientation: N-S. The stones were fixed with a fiercely burnt red clay plaster. The same material covered the grave. When we opened it, we thought at first that the plaster covered the bottom of the grave. In the $\mathrm{S}$ end of the grave, above the plaster, fragments of a glass beaker, calcined bones and small pieces of bronze plate were found. After opening the grave we realized that the plaster constituted the top of the grave. We found ashes below the $3-5 \mathrm{~cm}$ thick brownish-red plaster and a fragment of a lid in the $\mathrm{N}$ end of the grave. In the Send of the grave, in the same level of plaster which covered the ashes, there was a flat block of stone. The fragments of the above mentioned glass beaker were placed on this stone.

Measurable L: $195 \mathrm{~cm}, W: 32 \mathrm{~cm}$. D: $340 \mathrm{~cm}$. Both the plaster and the stones constituting the walls of the grave were severely burnt. Below the plaster covering the grave ashes were found in a 8-10 cm thick black layer. There were very few bone fragments. The bottom of the grave was of clay, mixed with sand, burned red. Above the plaster, covering the ashes, the grave was filled with yellow clay.

Associated articles, from the top of the grave: 1. Fragment of vessel. Brick-coloured, with red paint. 55.35.1.

2. Fragments of vessel. Two pieces. Coarse, grey side fragments. 55.35.2.

3. Pieces of plaster. Burnt. 55.35.3.

4. Calcinated human bones. 55.35.4.

5. Pieces of charcoal. 55.35.5.

6. Fragment of lid. Coarse, grey, conical. Diam: 14.5, Diam. of button: $6.7 \mathrm{~cm} .55 .35 .10$ (Pl. 33, No. 5).

In the southern end of the grave:

7. Glass beaker. White, conical. Complete. H: 12, DM: 8.6, DB: 3.3 cm. 55.35.13 (Pl. 33, No. 4).

8. Fragments of vessel. Two pieces. Of a grey household vessel; one of them is a small fragment of a base. 55.35.11 (Pl. 33, No. 3).

9. Glass fragment. Piece of rim. 55.35.12.

10. Glass fragments. Three pieces. Different types, one of them is part of a rim. 55.35.14 ( $\mathrm{Pl}$. 33, No. 6).

11. Fragments of glass beaker. Five pieces. Brown pieces of bottom and rim. Burnt. 55.35.15 (Pl. 33, No. 7).

It was possible to establish that the pile of stones resembling a wall on the $\mathrm{W}$ face of the trench, at a D. of $320 \mathrm{~cm}$ with a Th. of $40 \mathrm{~cm}$, was a row of stones assembled without mortar. Behind it there was yet again a layer of burnt clay, indicating the location of the next cremation burial.

\section{0, RĀKóCZI ROAD}

Below the road surface between the University and Malom Street there were a few skeletons without articles. ${ }^{176}$

\section{BAJCSY -ZSILINSZKY (SIKLÓSI) ROAD CENTRAL CEMETERY (Suppl. 1)}

Grave $\mathbf{R} / 227$. The following was found during an emergency excavation:

Jet bangle. ${ }^{177}$ of $\mathrm{D}$ cross section, smooth, broken. Black. Diam: 5.2, W: 0.6, Th: $0.3 \mathrm{~cm}$. 1305/1939 (Pl. 26, No. 4).

\section{8, BAJCSY-ZSILINSZKY ROAD (Suppl. 2)}

Grave R/228 (?). In April 1900, while an ice-pit was being dug, a tombstone was found at a $\mathrm{D}$. of $150 \mathrm{~cm}$ :

1. Tombstone with inscription. ${ }^{178}$

2. Tombstone with relief, fragment. ${ }^{179}$

\section{BAJCSY-ZSILINSZKY ROAD (Suppl. 2)}

Grave $\mathbf{R} / 229$. $280 \mathrm{~cm}$ below the road surface a brick grave ${ }^{180}$ was found; it was built of brick. Disturbed remains of a skeleton. D: $180 \mathrm{~cm}$. (A. r: II.1927.165/28.)

According to the entry in the A. r. the following objects, which may have belonged to the above grave, were found at a D. of $200 \mathrm{~cm}$ :

1. Bronze bowl. Diam: 7, rim: $1 \mathrm{~cm}$. NI.

2. Pottery fragments. Fragments of the base of a pottery flagon, three pieces; clay lid of a vessel, one piece; base of a jug, one piece. (A.r. 1927.73/14 $-15)$. NI.

\section{7, BAJCSY - ZSILINSZKY ROAD}

In the courtyard:

Coin. Roman, PB, stray (A. r. IV. 1940/1966). NI.

176 Gosztonyi $I$, p. 87. As their date of origin is problematic, we have dispensed with their numbering.

177 W. Hagen, Kaiserzeitliche Gagatarbeiten aus dem rheinischen Germanien. Bonner Jb. 142(1937) p. 110, Typ 4. Taf. 21. B. 4,1.

178 Stone Finds, Cat. No. 58.

179 Stone Finds, Cat. No. 52.

180 Dunántúl, 17 April 1927; Gosztonyi I, p. 112, Grave LXXII. 


\section{SOUTHERN PART OF \\ BAJCSY-ZSILINSZKY ROAD}

Grave $\mathbf{R} / \mathbf{2 3 0}$ (?). The following was found near the railway station, during earthworks:

Bronze brooch. Three-knobbed, crossbow type. With twisted wire on its foot and bow. On two sides of the foot 10-11 pseudo-pelta ornaments. With safety device. L: 9, W: $5.6 \mathrm{~cm}$. 55.40.1. (Pl. 43).

\section{CORNER OF MAJLÁTH STREET AND BAJCSY-ZSILINSZKY ROAD (Suppl. 2)}

Grave R/231. In 1928, during the drainage works, a $150 \mathrm{~cm}$ long sarcophagus was found. ${ }^{181}$ There was a skeleton inside.

Graves R/232-233. In the same area, during drainage works, two more Roman graves were found at a few metres' distance from one another. In Grave R/232 a gold bracelet and a pottery vessel were found. NI.

Grave R/233. Intact. According to the extent records ${ }^{182}$ the grave was to be opened in the presence of experts. (Nothing further is known about it.)

\section{9-21, MAJLÁTH STREET (Suppl. 2)}

Grave R/233/a. Gy. Török found an early Roman layer with a grave made of stone at a D. of 100-180 cm during the excavations he conducted. ${ }^{183}$ Orientation was approximately NE-SW. The northern portion was demolished, and its dimensions were not noted. Gy. Török mentions a plinth of a tombstone near the grave, but it is not indicated on his drawing. He also mentions other graves near the present one. The articles found in the graves are not described.

\section{SZALAI ANDRÁS (REGÖS) STREET (Suppl. 2)}

Graves R/234-237. In 1940 a new street was opened between Benyovszky Móric Street and Alsó Malom Street and a new network of sewers and water supply pipes was constructed through

181 Dunántúl, 12 June 1928; JPM-Reference Archives, 239.

182 Dunántril, 13 June 1928; JPM-Reference Archives, 229

183 Gy. Török, $P M E$ (1942) p. 20. the courtyards. Here a part of a Roman cemetery was cut through, and its graves, threatened by destruction, were saved by the Municipal Museum. ${ }^{184}$ But several graves had become inaccessible due to the road construction.

To the $\mathrm{N}$ the cemetery had stretched to the courtyard of the "Sopiana Engineering Works", where four more Roman tombs were found (1-4). Earlier, in the trench of the sewer, six graves were found $(5-10=$ Graves $\mathbf{R} / \mathbf{2 3 8}-\mathbf{2 4 2})$, of which five have an orientation NE-SW, head to the NE; one of them was a brick grave, and the orientation of this grave (Grave R/243) was SW-NE, head to the SW. An iron sword was lying beside it. The archaeologist conducting the excavation thought the grave belonged to the Migration Period or to the Middle Ages. D: $260 \mathrm{~cm}$.

Articles found in the graves:

Coin: Valens AE 3 (C-XII. 52).

Pottery beaker. Brick-coloured, with three handles. Greenish-brown glaze outside. H: 8, DM: 6.5, DB: $2.8 \mathrm{~cm}$. (A. r. IV. 1212-1216/1941.) (Pl. 34, No. 4).

On 26 October 1940, during the construction of the water pipe, a new grave was found with NE-SW orientation. Head to NE. ${ }^{185}$

Grave R/244. Earth grave. D: $155 \mathrm{~cm}, \mathrm{~L}: \mathbf{1 7 5}$, $\mathrm{W}: 60 \mathrm{~cm}$. A supine extended female skeleton was in the grave. Both forearms were resting on the pelvis. L. of skeleton: $150 \mathrm{~cm}$. On its right there was the supine extended skeleton of a child, the arms again on the pelvis. L. of the child's skeleton: $70 \mathrm{~cm}$.

Associated articles:

1. Pottery flagon. Brick-coloured. With a flat ribbon handle, and a rather worn, brownish-yellow glaze outside. H: 14.5, DM: 3.8, DB: 5 cm. 7190-1 (Pl. 34, No. 5).

2. Bronze earring. Made of thin wire, open. It has a loop and hook fastening and a movable pendant to the end of which an elongated bead of green glass paste is attached. Incomplete, fragmented. (The second member of the pair has corroded away to one of the beads described at No. 4.) Diam: 1.9, Th: $0.2 \mathrm{~cm} .7190-2$ (Pl. 34, No. 3).

3. Glass beads. Six items of light green glass paste, small cylinders; one is of dark blue glass in prismatic shape; one is lentil-shaped in dark blue glass; six items are small and rounded, made of white glass paste; two are small, of dark blue glass; one is a small dark green glass item; one is of white ribbed

\footnotetext{
${ }^{184}$ Report of A. Radnóti: HNM Reference Archives V. 105/1968; Gy. Török, PMÉ (1941) p. 5. 185 Radnóti, ibid.
} 
glass; three items are of coral (?), white beads of cylindrical shape. 7190-3 ( $P l .34$, No. 1).

4. Beads. Four items are large and rounded of black and dark blue glass paste with marvered white decoration; one is similar, large, elongated, prismatic and of dark blue glass; one is large, flat, rounded and of dark blue glass; two are small light green glass cylinders; one is an elongated, prismatic amber bead. $7190-4$ ( $P l .34$, No. 2).

Grave R/245. On October 28, 1940 another grave was found in the trench for the water mains. ${ }^{186}$ D: $150 \mathrm{~cm}$. Orientation: SW-NE. Head to the SW. L. of grave: $170, \mathrm{~W}: 45 \mathrm{~cm}$. Inside there was a supine extended female skeleton, with both lower arms on the pelvis. There were no associated articles in the grave. According to the archaeologist conducting the excavations this was a grave from the Migration Period or from the Middle Ages as its orientation differs from the Roman tombs. At the skull of the skeleton there were large stones haphazardly laid, which had pressed the skull forward (A. r. IV. 1212-1216/1941).

\section{CORNER OF RÓZSA FERENC (ALSÓ MALOM) AND ZSINKÓ I. STREET}

(Suppl. 2)

Grave $\mathbf{R} / 246$. This was found during drainage works. ${ }^{187}$ D: 130, W: 75, H: $120 \mathrm{~cm}$. The wall of the grave is $15 \mathrm{~cm}$ wide, made of stone with strong lime mortar. The roof was made of large-size bricks $(42 \times 29 \times 6 \mathrm{~cm}): \wedge$. The top had collapsed. Orientation: E-W. Inside there was a child's skeleton.

\section{KOSSUTH LAJOS (MAJLÁTH) SQUARE}

(Suppl. 2)

On October 21, 1926, during drainage works, several Roman bronze coins, pottery flagons and glazed pottery bowls were found at a D. of $194 \mathrm{~cm}$ in front of Hotel Vadászkürt. ${ }^{188}$ There were poultry bones in the bowls. According to Gy. Fejes the graves were disturbed (A. r. 1926. 191.7).

Grave R/247. Gy. Gosztonyi mentions a Roman brick grave which was found in front of Hotel Vadászkürt. ${ }^{189}$ Inside there were bones and one

\footnotetext{
186 Radnóti, ibid.

${ }^{187}$ Dunántúl, 14 April 1928; JPM-Reference Archives, 228.

188 Gy. Fejes, Dunántúl, 25 December 1926.
}

coin of Antoninus Pius (A. r. 1927/82 and 1926/191. 7).

During the drainage works of $1926^{190}$ the following were found:

Roman coins: Aurelian, Gallienus, Probus. (A. r. 1926.191.7.) NI.

Bronze brooch. Pelta-shaped. With spiral spring mechanism. Its pin is broken. L: $3.9, W: 3.1 \mathrm{~cm}$. 1290/1939 (Pl. 4\%, No. 6).

In 1947, during the drainage works in front of Hotel Vadászkürt the following was found at a D. of $140 \mathrm{~cm}$ :

Iron object, with square cross section. One end is flattened with a small protruberance, the other one is hooked. It may have been an angle or a key. L: 21.5, Th: $0.6 \mathrm{~cm} .1113$ (Pl. 47, No. 4).

\section{1, KOSSUTH LAJOS SQUARE (Suppl. 2)}

Grave $\mathbf{R} / 248$. This was found while the transformer station was being installed:

Fragments of a necklace. Five pieces. Made of bronze with attached beads, and a fastener in two pieces. L: $2.6,4.1,4,3.6$ and $3.7 \mathrm{~cm} .58 .32 .1$ ( $P l .43)$.

\section{CORNER OF BERCSÉNYI STREET AND RÁKÓCZI ROAD}

The following was found during drainage works on September 16, 1926:191

Coin: Vespasian (A. r. 1926/191.7). NI.

\section{4, PERCZEL STREET (Suppl. 2)}

The following were found during drainage works in 1926:

1. Bronze brooch. Three-knobbed, crossbow type. Diagonal lines on its bow, decorated with six pairs of punched ringlets on the foot. Intact. L: 10, W: $6 \mathrm{~cm} .1183$.

2. Bronze brooch. Three-knobbed, crossbow type. A double twisted filament decoration on its bow and 14 dotted circular decorations on the foot. With safety device. Intact, the pin is missing. L: 7.3, W: $5.3 \mathrm{~cm} .1201 / 16$.

Graves R/249-250. In 1926 Gy. Fejes mentioned two Roman graves in front of the house at 14,
${ }^{189}$ Gosztonyi I, p. 110, Grave XV.
190 Dunántúl, 19 September 1926.
191 Dunántúl, 19 September 1926. 
Perczel Street, behind the theatre. ${ }^{192}$ One of them was a sarcophagus made of mortar with an earth floor and a skeleton inside; the other was of brick, and a brooch, a spearhead and bones were found inside.

According to the entry in the A. r. for November 10, 1927, Gy. Fejes found a grave in the same place, at a $\mathrm{D}$. of $170 \mathrm{~cm}$, with a complete female skeleton.

Associated articles:

1. Bronze brooch. Three-knobbed, crossbow type, one knob is missing. With richly ornamented foot. L: $8.6 \mathrm{~cm} .1182 . \mathrm{E}$.

2. Coins:
(a) Gallienus
Ant.
(C-XII. 53)
(b) Gallienus
Ant.
(C-XII. 54)
(c) Gallienus
Ant.
(C-XII. 55)

3. Bronze earrings. Two items. According to the drawing in the A. $r$. they were made of thin bronze wire, forming an open ring with a pendant of three balls. M.

\section{4, PERCZEL STREET (Suppl. 2)}

Grave R/251. The following were found by J. Dombay during the rescue excavations in 1962:193

1. Iron blade of knife. Its back and edge are straight, curving at the point. With a tang resembling a nail. In good condition. L: 14.8 , L. of blade: 10.7, W. of blade: $1.9 \mathrm{~cm}$. 62.304.1 (Pl. 42, No. 1).

2. Arrowhead. Of iron. Elongated, trapezoid in shape, with the fragment of its tang resembling a nail. Incomplete L: $7.5, \mathrm{~W}: 2.4 \mathrm{~cm}$. In good condition. 62.304.2 (Pl. 42, No. 2).

3. Whetstone. Light grey in colour. L: $9, \mathrm{~W}: 2.7$ cm. 62.304.3 (Pl. 42, No. 3).

\section{CORNER OF PERCZEL AND GÁBOR STREET (Suppl. 2)}

Grave $\mathbf{R} / \mathbf{2 5 2}$. The following were donated to the Pécs Museum in 1961:

1. Fragment of iron dagger. With a wide blade and a fragment of tapering tang. Incomplete L: 19, W. of blade: $5 \mathrm{~cm}$. Broken, incomplete. 62.306.1 (Pl. 42, No. 1).

2. Fragment of vessel. From a dark grey cup. DM: $10 \mathrm{~cm}$ (?). 62.306 .2 (Pl. 42, No. 2).

192 Gy. Fejes, Dunántúl, 25 December 1926; Gosztonyi I, p. 111, Graves LX-LXI. In the second grave he also mentions three coins of Gallienus.

${ }^{193}$ There is no note relating to this in the Reference Archives of the HNM, but it can be established on the basis of the entry in the Inventory of the Pécs Museum.

\section{PERCZEL STREET}

Without closer indication of the site, the following was found during the drainage works in 1926:194 Coin: Traian (A. r. 1926/191.7). NI.

\section{4, FELSÓ-MALOM STREET (Suppl. 2)}

Grave R/253. In 1927 during drainage works Gy. Fejes found eight Roman bricks $(39 \times 30 \times 5 \mathrm{~cm})$ and several pieces of roofing tile at a. D. of $160 \mathrm{~cm}$ (A. r. 1927/211-9).

\section{3, KOSSUTH LAJOS (KIRÁLY) STREET}

(Suppl. 2)

Grave R/254. In 1870, when gas pipes were being laid down in Király Street, a Roman brick grave was found in front of the Aranyhajó Restaurant. ${ }^{195}$

In 1924 a human skull was found in Király (Kossuth Lajos) Street during drainage works, but its exact location was not noted. 196

\section{6, KOSSUTH LAJOS STREET}

In the courtyard of the treasury building (ärarisches Gebäude) a fragment of a $38 \mathrm{~cm}$ high marble relief was found. ${ }^{197}$ It represented the torso of a boy wearing a chlamys on his right shoulder and holding a basket in his left hand. M.

\section{3, JÓZSEF STREET (Suppl. 2)}

Graves R/255-256. During drainage works two Roman graves were found in front of the house. ${ }^{198}$ D: $220 \mathrm{~cm}$. They were disturbed and without associated articles.

\section{8-20, JOZZSEF STREET (Suppl. 2)}

Graves R/257-258. In 1927 during drainage works two disturbed graves were found in front of

${ }^{194}$ Dunántúl, 19 September 1926.

195 A. Horváth, Arch. Ért. (1870) p. 105; Baranya II, p. 195; Szönyi I, p. 10; Gosztonyi I, p. 106, Grave XVI.

196 JPM Reference Archives, 237. 159

197 W. Kubitschek-E. Loewy: $A E M$ III (Wien 1897) p.

198 Gosztonyi I, p. 113, Graves LXXX-LXXXI. 
the building (Angster Organ Factory). ${ }^{199}$ D: $210 \mathrm{~cm}$. There were disturbed skeletons, without articles (A. r. 1927/329/3).

\section{JÓZSEF STREET (WEST OF THE CORNER OF KIS FLÓRIÁN STREET) (Suppl. 2)}

Grave R/259. During drainage works a disturbed brick grave was found. ${ }^{200} \mathrm{D}: 200 \mathrm{~cm}$.

\section{8, ANNA STREET (Suppl. 2)}

Graves R/260-261. During the drainage works, Gy. Fejes excavated two graves on 23 March 1927.201 D: $200 \mathrm{~cm}$. The bottom of the graves was made of brick, the walls were of brick laid edgeways. These were leaning outward at the top. W. below: 30 , on top: $40 \mathrm{~cm}$. The graves had flat lids of seven Roman bricks. Inner H. of graves: $40 \mathrm{~cm}$. Orientation: $\mathrm{E}-\mathrm{W}$. In Grave $\mathrm{R} / 260$ there was a male skeleton; L. of grave: $166 \mathrm{~cm}$; in Grave $\mathrm{R} / 261$ there was a female skeleton, $\mathrm{L}$. of grave: $157 \mathrm{~cm}$.

\section{Grave R/260.}

Associated articles:

1. Bronze brooch. Three-knobbed, crossbow type. Its bow and foot are ornamented with plaited bronze wire. Intact, the pin is missing. L: 9.1, W: 5.3, Th: $0.5 \mathrm{~cm}$. 1115/1939 (Pl. 35, No. 1).

\section{Grave $\mathbf{R} / \mathbf{2 6 1}$.}

Associated articles:

1. Glass ball. Dark blue, with a small handle on its bottom, and irridescent surface. Diam: $3.8 \mathrm{~cm}$. 1120/1939 (Pl. 35, No. 2).

2. Glass ring. May have been a bangle (A. r. 1927/154. 12-14). NI.

3. Bone comb. Two rows of teeth; both ends are plain, without decoration, and of angular shape. Its front and back side are strengthened by a bone rib $8.9 \mathrm{~cm}$ long and $1-1.5 \mathrm{~cm}$ wide, which is fastened by five bronze rivets $0.8 \mathrm{~cm}$ long. On the front side, the edges of the rib are bordered with engraved lines, and each of the two ends with five

199 Notes of Gy. Fejes: JPM-Reference Archives, 210; Gy. Fejes, Dunántúl, 25 December 1927.

200 Cf. note 199.

201 Notes of Gy. Fejes: JPM-Reference Archives, 220. Gy. Fejes. Dunántúl, 17 April 1927; Gosztonyi I, p. 112, Graves LXX-LXXI. transverse grooves. Broken into several pieces..202 L: 11.2, W: 4.2, Th: $0.8 \mathrm{~cm} .925$ (Pl. 35, Nos 3a-b). - With this find fragments of green glass beads were also found.

According to O. Szőnyi, ${ }^{203}$ at an earlier date, while a house was being built in Anna Street, Roman coins and water pipes were found.

In the same place, at a D. of $590 \mathrm{~cm}$ a black glazed flagon, with wavy line decoration and narrow neck was found, and inside there were a few grains and burnt wooden chips. 1672 .

In the same place was found a pottery vessel which tapered downwards. 2749.

At the corner of Anna and Nagy Flórián Streets, during the construction of the Dóczi house, a small Roman bronze coin and vessels were found in the earth.

Coin: Valens AE 3 (C-XII. 56).

\section{0, ANNA STREET (Suppl. 2)}

Grave R/262. In front of the building at 20, Anna Street a grave was found, according to Gy. Gosztonyi's note from 1927.204 No further details were recorded.

\section{KULICH GYULA (PAPNÖVELDE) STREET}

Pottery dish. Glazed, decorated with notches. 1.1948.22. The circumstances under which the object was found are unknown.

\section{4, ERZSÉBET STREET}

The following were donated to the Pécs Museum: Coins:
(a) Constantine I
PB (C-XII. 57)
(b) Julian
AE 3 (C-XII. 58)
(c) 4th century
PB
M.

${ }^{202} D A C L \mathrm{XIII} / 2$, p. 2935; F. Fremersdorf: Das fränkische Reihengräberfeld Köln-Müngersdorf, I-II, Berlin, 1955 p. 144, Taf. 127/6; p. 145, Taf. 127/4; Ae. Kloiber: Die Gräberfelder von Lauriacum. Das Ziegelfeld, Linz, 1957, p. 91, Taf. XLVII/1; G. Gomolka, Klio, 47/1967, p. 299, Taf. IX. No. 62; in Pannonia: J. Hampel: Alterthümer des frühen Mittelalters in Ungarn, I-II, Braunschweig, 1905, I. p. 268, Fig. 636 (Keszthely); p. 268, Fig. 637 (Bezenye); L. Barkóczi: Későrómai temetó Pilismaróton (A Late Roman Cemetery at Pilismarót). $F^{\prime} A 12(1960)$ p. 117, 32. Fig. 1, Pl. XXII. No. 11. ${ }^{203}$ O. Szőnyi, $P B M E$ (1912) p. 161.

204 Dunántúl, 17 April 1927; Gosztonyi I, p. 112. Grave LXIX. 


\section{RUDAS LÁSZLÓ (GRÜNDLER) STREET \\ (Suppl. 1)}

Graves R/263-264. In 1941, during drainage works, two Roman tombs and three graves from the Migration Period were found. ${ }^{205}$ Roman graves:

\section{Grave R/263.}

Associated articles:

1. Pottery beaker. Dark grey, granulated. Its handle is broken. L: 10.4, DM: 9.5, DB: $5 \mathrm{~cm}$. 3193/1941 ( $P l .36$, No. 1).

2. Pottery bowl. Grey, fragmented. Size: $4.3 \times$ $\times 17.4 \mathrm{~cm}$. 3194/1941. E.

Grave $\mathbf{R} / \mathbf{2 6 4}$. There was an undisturbed male skeleton. Orientation: N-S. L: 200, W: $70 \mathrm{~cm}$. D: $250 \mathrm{~cm}$.

Associated articles:

1. Bronze brooch (at the left shoulder). Crossbow type, T-shaped, with decoration on the foot. L: $8.4, W: 6 \mathrm{~cm}$. 3440/1941. M.

2. Bronze buckle (at the pelvis). With D-shaped loop, movable tongue and a rounded plate, the front side of which is ornamented with three rivets and three dotted circles. L: $3.1, \mathrm{~W}: 2.2$, Th: $0.4 \mathrm{~cm}$. 3441/1941 (Pl. 36, No. 2).

3. Iron scissors (by the left thigh bone). One half is broken. Incomplete. L: 35.5, L. of shank 15, W: 5, Th: $0.3 \mathrm{~cm}$. 3442/1941 (Pl. 36, No. 4).

4. Iron slicer (?) (by the left femur). This is an ornate plane wrought from a solid handle and the edges bent back. The tang is arched and stands out from the handle. L: 29, L. of handle: $14.7, \mathrm{~L}$. of plane: 12.5, W: $5.8 \mathrm{~cm}$. 3443/1941 ( $P l .36$, No. 5).

5. Glass bowl (by the left femur). Light green, thin-walled, slightly conical. Restored. ${ }^{206} \mathrm{H}:$ 4, DM: 20, DB: 5 cm. 3445/1941 (Pl. 36, No. 3).

6. Coin (at the pelvis):

Constans PB (C-XII. 59).

\section{4, ISTVÁN STREET}

A coin was found while the vineyard was being cultivated:

Constans PB (A. r. 1910/109). NI.

\footnotetext{
${ }^{205}$ Gy. Török, $P M E$ (1941) p. 4; In the Inventory of the Pécs Museum "three Avar graves" are mentioned. On them ef. Fülep $V$.

${ }^{206}$ Isings, p. 144. Form 116; Benkő, p. 126. Typ 8/a, No. 3 (in our copy); Burger I, p. 101. No. 19 for further literature, Grave 2, p. 9. Fig. 194.
}

\section{MÁTYÁS FLÓRIÁN STREET}

Coin:

Valentinian I - Valens - Gratian (?)

Gloria Romanorum. Siscia. 157/1914-2.

\section{1, TETTYE STREET (Suppl. 2)}

Grave $\mathbf{R} / \mathbf{2 6 5}$. The following was found during a rescue excavation:207

Glass bangle. Oblong in cross section, with greenish-yellow paint on black. L: 3, W: $0.6 \mathrm{~cm}$. 62.317.3 ( $\mathrm{Pl} .42)$.

\section{TETTYE}

Coin. (A. r. 894/1939). NI.

\section{SIPTÁR VINEYARD}

Coin:

Valentinian I (?) AE 3 (A. r. 194/1913).

\section{CUSTOMS HOUSE ON THE ROAD \\ TO BUDA (Suppl. 1)}

Graves R/266-273. In 1870, near the Customs House of Buda, three Roman graves were found in the quarry. The graves were cut into the soft white limestone. There was no roof on the graves. According to A. Horváth ${ }^{208}$ the graves were destroyed, which is why there was no covering on them.

\section{Grave R/266.}

Associated articles:

1. Bronze bangle. Made of wire. NI.

2. Bronze bangle. Open; its ends are flattened and ornamented with two imprinted dots each. Diam: $6 \mathrm{~cm}$. NI.

3. Bronze ring. Made of wire; one flattened end is missing. Diam: $2.4 \mathrm{~cm}$. NI.

4. Fragment of bronze wire. Rounded. NI.

Grave $\mathbf{R} / \mathbf{2 6 7}$.

Pottery vessel, black. H: 13.5, DM: 10.5, DB: $5.8 \mathrm{~cm}$.

207 Report of A. Kiss: HNM Reference Archives, XII. 131/1966.

208 A. Horváth, Arch. Ért. 3 (1870) p. 187; Baranya II, p. 195; Gosztonyi I, p. 106 mentions 6 graves: Graves XVIII-XXIV(?). A. Horváth also speaks about 'new' graves that were found near the previous ones. 
Grave $\mathbf{R} / \mathbf{2 6 8}$. This was not opened, for reasons not indicated. According to A. Horváth and F. Várady five of the graves were to the $\mathrm{E}$, one lay "transversely". The dimensions and D. are unknown.

Associated articles according to B. Posta:209

1. Glass bottle. Its neck was broken. NI.

2. Pottery beaker. Yellow glazed, the handle is broken. NI.

3. Coin: "Iul. Val. Constantine's copper coin., Fel. Tem. Reparatio." NI.

Under the entry in the Inventory of the Hungarian National Museum for 28 June 1871 the material from seven graves, found in Pécs and dorated by Károly Piacsek and Ferenc Petz, may be found. ${ }^{210}$ (Graves 2-8; there is no mention of Grave 1.) On the basis of the name of the donor, the glass bottle, the yellow glazed flagon with handle, and the coin, these graves can very probably be identified with the ones found on a second occasion near the Customs House of Buda; there, however, according to the finds, eight graves were found altogether during the second investigation, and not only three. The graves were situated close by one another. The finds are the following, given in the order of the graves from $\mathrm{E}$ to $\mathrm{W}$ :

Iron blade of dagger, with hilt, $11.5 \mathrm{~cm}$.

Grave $\mathbf{R} / \mathbf{2 6 9}$.

Coin: Constans AE 2 (C-XII. 61).

\section{Grave R/270.}

Pottery vessel, black. H: 9, DM: 8, DB: $5 \mathrm{~cm}$.

\section{Grave R/271.}

1. Yellow glazed flagon with handle. H: 25, DB: $6 \mathrm{~cm}$.

2. Bronze fastener, grooved on its arched part.

3. Small silver ringlet.

To the N:

\section{Grave R/272.}

1. Pottery flagon. Brownish-grey, coarsely granulated. Made by hand. It has vertical burnished lines outside. H: 17.5 , DB: $7.5 \mathrm{~cm}, \mathrm{DM}: 7.3 \mathrm{~cm}$. HNM. 1871/165.8 (Pl. 26, No. 11).

2. Transverse part of iron dagger.

3. Iron knife blade. Leaf-shaped. Single edged. Its tang and point are broken. L (incomplete): 5.8, W: 2.4, Th: $1 \mathrm{~cm}$. HNM 1871/165.9 (Pl. 26, No. 9 ).

\footnotetext{
209 Baranya II, p. 195.

210 HNM Inv. No. 163/1871.
}

4. Iron buckle. D-shaped, with square cross section. The tongue is broken, and the loop is incomplete. H: 4, W: 3.2, Th: $0.7 \mathrm{~cm}$. HNM 1871/165.9 (Pl. 26, No. 10).

5. Iron stylus and iron fragments.

6. Pieces of glass vessel.

In the northern row:

\section{Grave R/273.}

1. Flagon with handle, brown glazed. H: 13.5, DM: 3.5, DB: $3.7 \mathrm{~cm}$.

2. Bronze stylus. L: $6.5 \mathrm{~cm}$.

3. Fragments of glass bottle.

4. Coins :
(a) Constantine I
AE 3 (C-XII. 60)
(b) Constans
AE 3 (C-XII. 62) (2)
(c) Constans
(d) Constans
AE 3 (C-XII. 63)
(e) Constantius II
AE 3 (C-XII. 64)
AE 3 (C-XII. 65)
(f) Constantius II
AE 3 (C-XII. 66)
(g) Constantius II
(h) Constantius II
AE 3 (C-XII. 67)
(i) Constantius II
(j) Const. Gallus
(k) Const. Gallus
(l) Const. Gallus
AE 2 (C-XII. 68)
AE 3 (C-XII. 69)
AE 2 (C-XII. 70)
AE 2 (C-XII. 71)
AE 2 (C-XII. 72)
(m) Const. Gallus
(n) Const. Gallus
AE 2 (C-XII. 73)
AE 2 (C-XII. 74)

\section{BASAMALOM LANE (Suppl. 1)}

Grave $\mathbf{R} / \mathbf{2 7 4}$. This was found in 1948 during soil cultivation in the vineyard; it is a Roman brick grave.

Associated articles:

1. A pair of bronze brooches, with an iron spring mechanism. 7110. M.

2. Bronze knee brooch with spring. Pin missing. L: $2.7, \mathrm{~W}: 1.7 \mathrm{~cm} .7111$.

3. Bronze bangle. Flat, thin, with $\mathrm{S}$ cross section. Penannular with flattened snake heads. Diam: 4.5, W: 0.2 , Th: $0.1 \mathrm{~cm} .7111$.

4. Bronze bangle. Solid, penannular, with buttends. Intact. Diam: 5.9, Th: $0.5 \mathrm{~cm} .7111$.

5. Ring. Of flat bronze wire, incomplete. 7111.

6. Bronze pin. May have been part of a brooch. 7111.

211 The above coins were registered in the Coins Collection of the HNM under 1871/1337, according to the definition of $\mathrm{Coh}^{2}$ VI. They were identified afresh on this occasion. - The survey of the Coins Collection completed in 1970 indicates that the pieces under C-XII. 67 and 69 were missing. 22 instead of 9 coins are mentioned in the detailed enumeration. 


\section{BUDA GARDEN CITY (DIÓS LANE, KALEF SETTLEMENT) (Suppl. 1)}

Grave R/275. On May 21, 1927, beside the highway to Pécsvárad, at the $\mathrm{N}$ end of Diós Lane, i. e., at the so-called Kalef Settlement, a Roman grave was found during the construction of a house. ${ }^{212}$ D: unknown. As the grave was found during the digging of foundations preceding the construction of the house, it could not have been very deep in the soil. L: 200, W: 52, H: $85 \mathrm{~cm}$. Brick grave, H. of side walls: $45 \mathrm{~cm}$. Its bottom is of brick with a $6 \mathrm{~cm}$ high cushion at the head. Its roof is a double $\wedge$; between the two rows of brick on the roof there was a space of $20 \mathrm{~cm}$. The upper roof was covered by an 8-10 cm thick layer of lime mortar. Orientation: E-W. May have contained a female skeleton.

Associated articles:

1. Pottery flagon. With a yellowish-green glaze. $\mathrm{H}: 12 \mathrm{~cm}$. NI.

2. Fragments of glass jugs. 32 pieces.

3. Pottery flagon, yellow glazed, broken. 3 pieces.

4. Bronze bands of a casket, four items.

5. Coins, 13 items ( 7 could be identified):
(a) Licinius
Foll. (C-XII. 75)
(b) Licinius
Foll. (C-XII. 76)
(c) Constantine
AE 3 (C-XII. 77)
(d) Constantine
AE (C-XII. 78)

6. Small iron rod. L: $15 \mathrm{~cm}$.

7. Silver coin. According to the description of Gy. Fejes ${ }^{213}$ this was of Maximianus Hercules; obverse: portrait of the emperor, reverse: two soldiers, legend: CONVERTOT AVGG (sic!).

8. Glass bangle.

9. Bronze bangle. Twisted of two wires, at each of the two ends is a bronze amphora-bead. With a hook and loop fastener. Diam: 6.3, Th: $0.7 \mathrm{~cm}$. 1669/1939 (Pl. 38, No. 4).

10. Bronze bangles. 4 items. Plain. NI.

11. Bronze hinged crossbow broch. NI.

12. Bronze pin. L: $10 \mathrm{~cm}$. NI.

13. Bone pin. According to the description by Gy. Fejes it was a "wooden pin". Yellow, broken in two. L: $10 \mathrm{~cm}$. 1556/1939 (Pl. 38, No. 5).

14. Links of gold chain. 6 small gold cylinders. At the two ends and in the middle they are furrowed and decorated with slanting cuts. The ends are perforated. L: 1.4, Diam: $0.6 \mathrm{~cm}$. Weight: $1.25 \mathrm{gr}$ (altogether). 957-2.1939.

15. Pendant from gold chain. Made of thin gold plate; small, cup-shaped. It narrows toward one end. With a collar on both ends, decorated with impressed dots and slanting lines. There is a soldered loop in its side. L: $1.8 \mathrm{~cm}$. Weight: $1.23 \mathrm{gr}$. 957-3.1939 (Pl. 38, No. 3).

16. Cornetian bead from chain. One item. Yellow. Size: $1.2 \times 1 \mathrm{~cm}$. 1116.1939 (Pl. 38, No. 2).

17. A pair of gold earrings. The setting is made of thin sheet with a mother of pearl (?) plaque inside. The gold wire ends are bent into " $\mathrm{S}$ " shape. L: $2 \mathrm{~cm}$. Weight: 2.63 gr. 957.1939 (Pl. 38, Nos la-b).

18. Bronze rings. Seven pieces. Made of thin plate and wire, respectively. One with bezel, two are broken. NI.

19. Bronze keys. Two items. The first has a ring on the top, the other is fragmented. NI.

20. Scent bottle. The rim is incomplete, the neck is cylindrical and the body widens slightly downwards. It has a foor. H: $6.4 \mathrm{~cm}$. 1142.1939. M.

21. Female jaw-bone. (A. r. 54.1928. 22-23).

22 . One piece of bone notched along the edge.

23. Fragments of wood from casket. Ten pieces. (A. r. 1928. 45-54.) NI.

According to the description by Gy. Fejes ${ }^{214}$ the casket was lying at the feet of the skeleton in the grave and may have been $10 \times 14 \times 20 \mathrm{~cm}$ large with nice bronze fittings. The fine bronze key with ring, found by the hand of the skeleton, may have belonged to this casket. (Here Gy. Fejes only speaks of one, not two, keys.) We know nothing about the position of the other finds in the grave.

\section{HOMOKBÁNYA LANE}

In 1899 in the "big" sand-pit the workers found four bronze vessels in an iron box of which $\mathrm{L}$. Juhász ${ }^{215}$ bought the iron box, three vessels and one "piercing knife", subsequently donated to the Pécs-Baranya County Museum.

According to J. Hampel ${ }^{216}$ the Hungarian National Museum obtained the finds directly from the collection of L. Juhász.

Iron box. L: 32.5, W: 29.5, H: $11.5 \mathrm{~cm}$. HNM 62.1902.5. M. - The three bronze vessels and one iron tool which follow were found in this box.

1. Bronze jug. Its body is bulbous, pear-shaped with a thin neck and round foot ring. Its mouth has a narrow lip to which the upper part of the curved handle, ornamented with leaves, was at-

${ }^{214}$ Gy. Fejes, ibid.

${ }^{215}$ L. Juhász, Arch Ért. (1901) p. 174.

216 J. Hampel, Arch. Ert. (1902) p. 431. 
tached.217 Below the mouth it is encircled by six rows of incised four-petal rosettes, and below them there is a garland of leaves. The upper portion of the body is covered with incised scenes, originally four altogether, but one of them was almost completely covered by the mask at the lower end of the handle. ${ }^{218}$ The scenes are the following:

(a) A running and jumping satyr holds a rhyton in his right hand; a panther is beside him. He pours the contents of the rhyton into the mouth of the panther. Behind him there is a large, serrated leaf which separates him from the other satyr.

(b) Heracles leaning forward in a tipsy manner, holding a club in his right hand. From the right a naked satyr supports him. Heracles' left arm is supported on the satyr's shoulder, his hanging hand holds a rhyton. Between the two figures there is an overturned mixing-bowl.

(c) A satyr leaning on a shepherd's crook on the ground; in front of him there is a rhyton, behind a syrinx. A rather faded Maenad, covered by the lower part of the handle, may have belonged to this figure. H: 23 , DM: 2.5 , DB: $6.5 \mathrm{~cm}$. HNM 62.1902.1 ( $P l$. 46, Nos 3-3a).

2. Bronze jug. With oval body, rounded base and a neck which tapers upwards and ends in a cornetshaped mouth. The handle is attached to the rim by two arms bent in an arc; between them is an upcurling leaf. The handle ends in a human foot attached to the body of the jug. The base is missing. ${ }^{219} \mathrm{H}: 27.5, \mathrm{DM}: 7.9, \mathrm{DB}: 7.5 \mathrm{~cm}$. HNM 62.1902 .2 (Pl. 46, No. 1).

3. Bronze jug. With a rounded, globular body, narrow neck, a pouring lip on the rim and a rounded base. Its big, strongly curved handle has a bulging edge on both sides. ${ }^{220} \mathrm{H}: 16.5$, DM: 4 , DB: $5.2 \mathrm{~cm}$. HNM 62.1902.3 (Pl. 46, No. 2).

4. Iron tool. It has a straight sharp blade with tangs at both ends. Scraper (?). L: $24 \mathrm{~cm}$. HNM 62.1902.4. M.

The above finds might have come from a hidden hoard or grave.

217 The lower part of the handle ended in a female mask. This is known only from the description in the Inventory; today only the place where it was fixed remains.

${ }^{218}$ L. Juhász, ibid.; J. Hampel, ibid.; G. Erdélyi: Arch. Ért. (1932-1933) p. 43 (dates it to the age of Caracalla); I. Paulovics: Arch. Ért. (1935) p. 93 and supra (dates it to the 2nd century); Radnóti $I$, p. 170 for further bibliography (considers it a 2 nd-century western product).

${ }^{219}$ Radnóti I, p. 167, XLIV, No. 1 (2nd-3rd centuries). ${ }^{220}$ Radnóti I, p. 151, L. No. 8 (3rd century).

\section{FELSZABADULÁS ROAD (THE HIGHWAY TO MOHÁCS) (Suppl. 1)}

Grave R/276. On 24 February 1938 a Roman brick grave was found in the grounds of the Deutsch Brick Factory. ${ }^{221}$ D: $310 \mathrm{~cm}$. The roof is made of bricks: $\Lambda$. L. of grave: $210, W: 45, \mathrm{H}: 50 \mathrm{~cm}$. Disturbed. In the grave there was the skeleton of a woman between the ages of 30 and 32 . There were no associated articles in the grave.

Near the Deutsch Brick Factory the following was found in 1941:

\section{Grave R/277 (?).}

Bronze bangle. Penannular, made of wire of D-shaped cross section. The ends are bent in on each other with stylized snake heads, and there are triple incisions on its side. Intact. Diam: 6.5, W: 0.4, Th: $0.3 \mathrm{~cm}$. 7125/1941 (Pl. 38, No. 6).

In 1959 the workers found and handed over the following from the Brick Factory of Felszabadulás Road, near the railway track.

\section{Grave R/278 (?).}

$A$ string of beads. 29 items are intact, 2 blue ones are broken: 9 are yellow, one is yellow with blue inlays (peacock pattern), a flattened, globular bead. 60.93 .1 ( $P l .42)$.

The following stray finds were given to the Pécs Museum in 1958 from the site of the Brick Factory without indication or more precise location:

\section{Grave R/279 (?).}

1. Bronze brooch. With a strongly bent profile, the head portion is long and the trunk is divided by two knobs. The catch-plate is perforated and the end of the foot is ornamented with a double knob. L: 7.5, W. of head: $2.7 \mathrm{~cm} .59 .33 .1$ ( $P l .42$, No. 1).

2. Bronze brooch. Winged, the head and back portion are decorated with a triple-ringed rib. The wing and the pin are missing. Distorted by fire. L: $4.5, W: 2.1 \mathrm{~cm} .59 .33 .2$ (Pl. 42, No. 2).

3. Bronze brooch. Knee brooch; its back, head and foot are severely distorted by fire. L: 4.4, W: $2.4 \mathrm{~cm} .59 .33 .3$ (Pl. 42 , No. 3).

4. Bronze brooch. Fragment. L: 5.9, W. of head: $3.2 \mathrm{~cm} .59 .33 .4$ (Pl. 42, No. 4 ).

5. Spindle-whorl. Clay, conical. On one side it has a tendril-like, impressed decoration. Brown. Diam: 3.5, Th: $1.6 \mathrm{~cm}$. 59.33 .5 ( $P l .42$, No. 5).

221 Rescue excavation of T. Horváth, report: HNM Reference Archives, X. 173/1968; Gosztonyi I, p. 115, Grave CXLIII. 
6. Bronze ring, fragment. Its bezel is rounded. On a round sheet, in a hollowed field, there is a dark blue stone with four red circles filled with yellow paste. Broken. Diam: 1.9, Diam. of head: 1 cm. 59.33.6 ( $P l .42$, No. 6).

7. Clay urn. Brick-coloured, made on the wheel. With a series of lines on its surface. H: 26, DM: 19.7, DB: $11.3 \mathrm{~cm} .59 .33 .7$ ( $P l .42$, No. 7).

8. Pottery jug. Light brown, made on the wheel. Broken, incomplete. H: 24.8, DM: 12 , DB: $8.5 \mathrm{~cm}$. 59.33.8 ( $P l$. 42. No. 8).

\section{ZELMS SAND-PIT (Suppl. 1)}

Grave $\mathbf{R} / 280$. In the 1880 s a Roman grave was found on the grounds of the Zelms sand-pit, along the highway to Üszög. ${ }^{222}$ The following objects were donated to the Pécs Museum:

1. Double bronze ring. NI.

2. Fragments of bronze bangle. Two pieces. Thin, with D-shaped cross-section. W: 0.3 , Th: $0.2 \mathrm{~cm}$. 2218/1940.

3. Fragments of iron knife. 2 pieces. With one edge and tang. L: 5.9, W: 1.1, Th: $0.4 \mathrm{~cm}$; L: 3.3, W: 1.2, Th: $0.2 \mathrm{~cm} .2217 / 1940$ (Pl. 38, Nos 7-8).

\section{POWER STATION (Suppl. 1)}

Graves R/281-289. Nine graves were found by P. Lakatos in 1956, during his rescue excavations. ${ }^{223}$ D: 200-220 cm. Orientation W-E. head to the W.

\section{Grave R/281.}

Associated articles:

1. Bronze brooch. With a spring, this belongs to the category of the so-called legionary brooch. L: $7 \mathrm{~cm} .56 .30 .1$ ( $P l .43$, No. 1).

2. String of bead. Consists of 32 blue, green and yellow glass beads. The blue and green ones are cylindrical, the light ones are rounded. 56.30.2 (Pl. 43, No. 2).

3. Bronze fragment. Resembling a pin. L: $2.3 \mathrm{~cm}$. 56.30 .3 .

\section{Grave R/282.}

Iron nails. Seven items. With hooked head and traces of wood fibre. L: $8.2 \mathrm{~cm} .56 .31 .1$ (Pl. 43).

${ }^{222}$ A. Marosi, $P B M E ́$ (1908) p. 60; Gosztonyi $I$, p. 107, Grave XXIX. He does not identify it with the grave found at the Zelms sand-pit.

${ }^{223}$ Report of P. Lakatos: HNM Reference Archives, 257. P. IV. P. Lakatos, Arch. Ért. 85(1958) p. 86. He dates the graves to the 2 nd century. Seven graves could not be identified (R/283-289).
Grave $\mathbf{R} / 290$. According to the record of $\mathrm{J}$. Dombay ${ }^{224}$ a grave containing a skeleton was found in the grounds of the Pécs Power Station in 1957, and the associated articles were "tvnical of the 4th century":

1. Pottery flagon. Light grey, made on the wheel. With one handle divided in the middle. H: 68 , DB: $4.2-6 \mathrm{~cm}$. 58.10.1 ( $P l .43$, No. 4).

2. Glass jug. Light greenish-blue, with a thicker wall and ribbon handle. Broken. DM: 5, DB: 3.5 cm. 58.10.2 (Pl. 43, No. 5).

3. Coin:

Trajan Decius GB (C-XVI. 3).

4. Glass bead. Light blue, a polyhedron. 58.10.5 (Pl. 43, No. 3).

5. Glass bead (?). Green, almond-shaped. It is not pierced. L: $1.6 \mathrm{~cm}$. 58.10 .6 ( $P l .43$, No. 3).

6. Gold bangles. 2 items. Twisted from two wires; the ends are upturned. Diam: 4.2-4.4 cm; 4-4.2 cm. 58.10.7 (Pl. 38, Nos 10, 12; Pl. 43, Nos 1-2).

7. Human bones. Three pieces. 58.10.4.

\section{TÁDÉ SIKORSKI'S BRICK-YARD}

(Suppl. 1)

Graves R/291-292. In 1908 "skeletons" were dvg out on the grounds of the Sikorski brick-yard, along the highway leading to Pécsvárad.225 The number of the graves is unknown. Gy. Gosztonyi registered two graves on the basis of the finds. ${ }^{226}$

Finds in the possession of the Pécs Museum:

1. Coin: Maximian. NI.

2. Coin: Constantine I. NI.

3. Bronze bangles. Two items. Cylindrical cross section; the bangles have a loop and hook fastener. Intact. Diam: 5.1, Th: $0.3 \mathrm{~cm}$. 1210-3/1939 (Pl. 38, No. 9).

4. Bronze bangle. With snake-head, penannular. Diam: $5.8 \mathrm{~cm}$. 1211-3/1939. Its drawing is extant. M.

5. Bronze bangle. Twisted of two pairs of wires, incomplete. Diam: $5.8 \mathrm{~cm}$. 1210-4/1939. M.

6. Bronze brooches. Two items. Crossbow types. L. of one: $4.7 \mathrm{~cm}$. 1211-1-2/1939. M.

7. Frame of buckle. Bronze, "8"-shaped. It was presumably the loop of a so-called "Omegabuckle”. Size: $3 \times 3 \times 2 \mathrm{~cm}$. 1211-5/1939. M.

8. Bronze buckle plate. Disc-shaped with two holes. Size: $3.5 \times 2.1 \mathrm{~cm}$. 1210-9/1939. Its drawing is extant. E.

\footnotetext{
224 J. Dombay, Arch. Ért. 85(1958) p. 203.

225 A. Marosi, PBMÉ (1908) p. 156.

${ }^{226}$ Gosztonyi I, p. 113, Graves LXXV-LXXVI.
} 
9. Bronze ring. Its head is missing. Diam: $2.1 \mathrm{~cm} .1211-4 / 1939 . \mathrm{M}$.

10. Bone plate. Pierced. Diam: $3 \mathrm{~cm} .1210-6 / 1939$. E.

11. Fragments of bone comb. Two pieces. Size: 2.3 and $1.9 \mathrm{~cm}$. M.

12. Iron axe. NI.

13. Glazed flagon. Yellowish-green. Its ribbon handle protrudes above the rim. DM: $-, \mathrm{H}: 29.7$, DB: $7.6 \mathrm{~cm} .119 / 1939.7$. M. NI.

14. Beaker with three handles. With yellow glaze.

15. Pottery beaker. Grey. H: 12.4, DB: $9.9 \mathrm{~cm}$. 1174/1939. Its drawing is extant. E.

16. Pottery beakers. Three smaller types. NI.

17. Glass beads. 1210-8/1939. E.227

\section{BRICK-YARD OF MRS SIKORSKI,}

\section{J. ZSOLNAY (Suppl. 1)}

Grave R/293. In 1912, by the highway leading to Pécsvárad, the following were found in the grounds of the brick-yard: ${ }^{228}$

1. Coin: Maximian (A. r. 1912/184). NI.

2. Cross-bow brooch. Of solid silver. Its foot is ornamented. Intact; the pin is also present. L: 5.4, W: $3.7 \mathrm{~cm} .654$ (Pl. 38, No. 11).

3. Bronze bangle. Of a narrow, thin strip. Open, incomplete. Diam: $5.1 \mathrm{~cm} .771$.

Gy. Gosztonyi mentions ${ }^{229}$ that behind the Zsolnay factory, on the Sikorski site, Roman graves and remains of buildings were found; in addition, he suspected a Roman villa in the vicinity.

\section{3, ALAJOS STREET (Suppl. 1)}

Grave R/294. During drainage works a Roman brick grave was found. ${ }^{230} \mathrm{D}: 150 \mathrm{~cm}$. Disturbed; there were no associated articles in the grave besides the few human bones found there.

The following was also found in Alajos Street: Coin: Roman PB, stray 966/1940. NI.

\footnotetext{
227 These objects were entered into the Inventory in 1939 from "old, unregistered material". A note in the Inventory shows that in 1939 it was felt that the articles were related to one another.

228 O. Szőnyi, PBMÉ (1912) p. 162; A. Graf, A Pannonia ókori földrajzára vonatkozó kutatások áttekintő összefoglalása (A Comprehensive Survey of the Researches Related to the Geography of Pannonia in the Classical Age). Diss. Pann. Ser. I, No. 5 (Budapest 1936), p. 117, Note 8.

${ }^{229}$ Gosztonyi $I$, pp. 92, 113.

230 Dunántúl, 8 August 1931.
}

\section{2, ATTILA STREET (Suppl. 1)}

Grave R/295-297. On September 25, 1928, three Roman graves were found during the drainage works in front of the Dermatological Clinic. ${ }^{231} \mathrm{D}$ : $300 \mathrm{~cm}$. It is unknown in which grave the associated articles were found.

1. Gold pendant. The ellipsoid frame was made of thin gold sheet, in which there may have been a stone (now missing). In the centre is soldered a loop made of narrow strip. L: $1.4 \mathrm{~cm}$. Weight: 1.01 gr. 799-1/1939 (Pl. 39, No. 1).

2. Bronze bangle. Narrow, flat. Its outside edge is milled and it is broken in two. Penannular; its ends are flattened. Diam: $6.5, \mathrm{~W}: 0.3$, Th: $0.1 \mathrm{~cm}$. 799-2/1939 (Pl. 39, No. 2).

3. Coin: Probus silver coin. NI.

\section{8, ÁRPÁD STREET (Suppl. 2)}

Grave R/298. Below the pavement in front of the building was found a Roman child's grave.232 D: 50, L: 142, W: 40, H: $35 \mathrm{~cm}$. Brick grave; besides the skeleton it did not contain any associated goods.

\section{1, ÁRPÁD STREET}

Coin: Valens - Securitas type. Stray. (A. r. 1915/61.)

\section{8, MAKÁR (TIBORC) STREET \\ (Suppl. 1)}

\section{Graves R/299-300.}

Two Roman brick graves were found in front of the building. ${ }^{233}$ Beside the bones no articles were found in the graves. Both are disturbed.

\section{4, MAKÁR (ALKOTMÁNY) STREET, BREWERY}

In 1956, while a pit was being dug in the courtyard of the brewery, the following was found:

Coin: Constantine II. PB (worn). 56.1.1.

${ }^{231}$ Notes of Gy. Fejes: HNM Reference Archives, 231/XIX, 1966; Dunántúl, 30 September 1928; Gosztonyi I, p. 115, Graves XC-XCII.

232 Dunántúl, 13 August 1926.

${ }^{233}$ Notes of Gy. Fejes: HNM Reference Archives, 231/XIX, 1966, 206; Dunántúl, 30 September 1928; Gosztonyi $I$, p. 114, Graves LXXXVIII-LXXXIX. 


\section{4, MECSEKALJA - ÚTTÖRŌ \\ (ALSÓ-MAKÁR) ROAD (Suppl. 1)}

Grave R/301. On April 23, 1963 a Roman brick grave was found while a lime-pit was being dug. ${ }^{234}$ D: $200 \mathrm{~cm}$. Both ends were open and the roof was made of bricks laid edgewise: $\Lambda$. The western part of the grave was entirely filled with earth. Orientation: $\mathrm{W}-\mathrm{E}, 50^{\circ}$ deviation from the $\mathrm{N}$. There was an adult female skeleton in the grave, without articles.

\section{8, NAGY JENŌ (KASZÁRNYA) STREET}

In front of the house, while gas pipelines were being laid, the following was found:

Glass unguent bottle. Bluish-green, prismatic. Its rim is broken. H: 4.7, DM: 2, DB: $2.7 \mathrm{~cm} .59 .28 .1$ (Pl. 47, No. 8).

\section{AIRPORT (RÁCVÁROS - TÉGLAGȲÁR) (Suppl.1)}

Grave R/302. On July 7, 1956, on the grounds of the old airport, during the construction of the new part of the city, a Roman grave was found. ${ }^{235}$ D: 150, L (inner): 155, W (inner): 34, H: $40 \mathrm{~cm}$. The longer side of the grave was built of four pairs of bricks laid edgewise. The bricks at the end of the grave were laid a little inward, so that both ends of the grave were narrower. It was covered by five bricks laid horizontally. The narrower ends of the grave were closed by one brick laid edgewise. Along the longer side of the grave, presumably to counterbalance the pressure of the earth, four bricks were laid vertically on each side beside the roof, but not touching it. The bottom of the grave was covered with 8 bricks. Disturbed. Orientation: W-E, head to the W. In the grave there was a male skeleton and the skull was at the $\mathrm{W}$ end of the grave. There were no associated articles.

\section{RÁCVÁROS}

The following was found in the lane behind the cemetery: $:^{236}$

Pottery beaker. Light grey, granulated. H: 8.7, DM: 8, DB: 4 cm. 843/1939 (Pl. 39, No. 7).

\section{MAKÁRALJA (Suppl. 1)}

Grave $\mathbf{R} / \mathbf{3 0 3}$. The following objects were added to the Juhász collection from the Roman grave on the grounds of the Ignorantine monastery:237

1. Iron ring. NI.

2. Ornamental iron belt (?), fragment. NI.

3. Crossbow brooch (?), bronze, hinged. NI.

\section{MAKÁRHEGY - MISETICS VINEYARD}

(Suppl. 1)

Grave R/304. In the collection of A. Horváth ("During the soil preparation in the vineyard it was found together with horse bones, a pottery beaker and pieces of iron."):

Bronze buckle. With a pelta-shaped loop, perforated plate and movable tongue; intact. L: 4.2, W: 3.2, Th: $0.3 \mathrm{~cm} .770 / 1939$ (Pl. 39, No. 8).

\section{MAKÁRHEGY - FOOT OF THE HILL}

(Suppl. 1)

Grave R/305. On May 18, 1931 the following were donated to the Pécs Museum:

1. Bronze brooches. Two items, knobbed. NI.

2. Blade of iron knife. L: 27, L. of handle: $11 \mathrm{~cm}$. (A. r. 1913/1943.1-2, 144/3). NI.

\footnotetext{
${ }^{236}$ Stone Finds, Cat. No. 11. Altar stone. - According to the landlord a cemetery was situated here.

237 Baranya II, p. 196.
}

\footnotetext{
234 Rescue excavation by A. Kiss, his report: HNM Reference Archives, IV. 54/1964.

${ }^{235}$ Rescue excavation by P. Lakatos, his report: HNM Reference Archives, 264. P. IV; P. Lakatos, Arch. Ért. $85(1968)$ p. 86.
} 


\section{ARANYHEGY (Suppl. 1)}

Grave $\mathbf{R} / \mathbf{3 0 6}$. In the vicinity of the vineyards of Pécs, on the so-called Aranyhegy, a marble sarcophagus with reliefs was found, ${ }^{238}$ together with a fragment of a tombstone with inscription. ${ }^{239}$

\section{CSERKÚT (Suppl. 1)}

Graves R/307-308. In 1907, on the hill of Cserkút, the following was found during soil cultivation for the vineyard:

1. Clay lamp. Its handle is broken and sooted. L: 7.9, DB: 3.9, H: $3.3 \mathrm{~cm} .670 / 1938$ (Pl. 39, No. 9). Also in 1907, in the vineyard of J. Herbert, the following were found in the cemetery which was considered at that time to belong to the "Migration Period":

\section{Grave R/307.}

1. Fragment of glass bangle.

2. Glass beads. Ten items, smaller ones.

3. Bronze fragments.

4. Glass fragments.

5. Rings, etc. (16 items).

6. Fragments of bronze earring. Two pieces of thin, cylindrical wire. It is a type with hook and loop fastener. 840 (Pl. 39, No. 5).

7. Jet bangle, fragment. Black, flat, with boss decoration. Fragment. L: 2, W: 0.8, Th: $0.4 \mathrm{~cm}$. 840 (Pl. 39, No. 4).

8. Glass bead. One piece. Green, prismatic. 840-2 (Pl. 39, No. 3).

9. Beads. Three light green, round ones (in the register ten were mentioned). 840/2.

10. Fragment of rim of glass beaker. White, thinwalled. 840-4 (Pl. 39, No. 6).

Grave R/308. The following objects were found on the hill:

${ }^{238}$ Stone Finds, Cat. No. 67; A. Sz. Burger: Római szarkofág a pécsi Aranyhegyről (A Roman Sarcophagus from the Aranyhegy of Pécs), Arch. Ért. 100 (1973), p. 42 and supra.
1. Bronze brooch. Crossbow type, three-knobbed. The central knob is missing. Twisted wire on the bow. With a safety device; the pin is missing. L: 7.8, W: $5.1 \mathrm{~cm} .853-1$ (Pl. 39, No. 12).

2. Bronze brooch. Crossbow type, three-knobbed. The left side has broken off. With four pairs of pelta ornaments on its foot, and a safety device. The pin is missing. L: $7.8 \mathrm{~cm} .853-2$.

3. Bronze brooch. Crossbow type, three-knobbed. The left side and the pin are missing. Twisted wire on the bow, four and six impressed dot ornaments on the foot. L: $7.8 \mathrm{~cm} .853-3$.

4. Spear head. Made of bronze, with a flat blade and short socket. The socket is pierced in two places. L: 14.4, Diam: $3 \mathrm{~cm}$. L. of blade: $8.1, \mathrm{~W}: 0.9-4.3$, Th: 0.3-1.7 cm. 853-4 (Pl. 39, No. 11).

5. Small bronze tubes. Two items. Astragal-like proportioned. Belt fitting. L: $4.5-4.6 \mathrm{~cm}$. 853-5 (Pl. 39, Nos 13a-b).

6. Fragment of bronze plate. L: 7.6, W: 1.7, Th: $0.1 \mathrm{~cm} .853-6$ (Pl. 39, No. 10).

7. Bronze button from an iron pin. Rounded, solid. Diam: $0.8 \mathrm{~cm}$. $853-7$.

8. Point of horn. Worked. L: $8.4 \mathrm{~cm}$. 853-8 (Pl. 39, No. 14).

In 1960 the following objects were bought by the Pécs Museum; they may possibly have come from Cserkút:

\section{Coins:}
(a) Claudius II.
AVR
(C-XVI. 5)
(b) Faustina
AVR
(C-XVI. 4)
(c) Constantius II.
AVR
(C-XVI. 6)

\section{DOKTOR SÁNDOR STREET (HIGHWAY TO SZIGET) (Suppl. 1)}

Graves R/309-314. In 1970 during construction works six Roman brick graves were found. They presumably had gabled roofs. Orientation: E-W. The alignment of the skeletons could not be identified. There were no associated articles. ${ }^{240}$

239 Stone Finds. Cat. No. 45.

240 The graves were excavated by Valéria Kováts. 


\section{KISKÖSZEG STREET (Suppl. 1)}

Graves R/315-317. Three brick graves were found on the hill during the road construction works. ${ }^{241}$ Roof: $\wedge$. E-W orientation. Disturbed.

\section{DISCHKA GYŌZZ̄ STREET (Suppl. 2)}

Grave R/318. During our excavations in the courtyard of the Post Office and in its vicinity an earth grave was found $\mathrm{W}$ of the main building under 10, Jókai Street, on the grounds of the socalled Kispark; it was in our trench 3, on the eastern side of wall $50 . .^{242} \mathrm{~L}$. of the grave: $140 \mathrm{~cm}$. The roof was made of 5 pairs of keyed bricks: $\Lambda$. The eastern end of the grave was beyond the trench. The grave was situated on a burnt layer containing charcoal and red-painted pottery fragments. There were fine pieces of lime mortar. When the fourth pair of bricks from the western end was removed a Roman coin came to light. Orientation: W-E, head to the W. (Pl. 40, No. 1$)$.

There was the supine extended skeleton of an elderly woman. The arms were bent at the elbow and placed on the pelvis. The skeleton was in rather poor condition. The bottom of the grave is at a level of $131.01 \mathrm{~m}$ above the sea level of the Baltic Sea. The level of the brick floor found in the SE corner of the trench is $131.97 \mathrm{~m}$.

The pieces of mortar found in the grave, as well as the orientation of the grave, indicate that the grave was dug among the ruins after the building was demolished. ${ }^{243}$

Associated articles:

1. Silver earring. A ring made of thin, cylindrical wire, beside the right ear. One of the pair is missing. Diam: 1.8, Th: $0.2 \mathrm{~cm}$. 70.6.1 (Pl. 40, No. 3).
2. Iron bangle, fragment (on the left lower arm). With textile fibres on it. Broken in two, incomplete. Diam: 6, Th: $0.8 \mathrm{~cm}$. 70.6.2 (Pl. 40, No. 2).

3. Coin: Valens AE 3 Aquileia 367-375, Securitas $\frac{*}{\text { SMAQS }}$; RIC 96.

Grave R/319 (Suppl. 2). In 1964, during our excavations in the courtyard of the Post Office, the terrazzo-floor was cut through in the $\mathrm{N}$ part of trench 8 and there were bricks of a disturbed Roman grave. It had a gabled roof. Fragments of human bones, glass vessels and of stone urn were found nearby.

Grave R/320 (Suppl. 2). In 1964, in trench 5 of our excavations conducted in the courtyard of the Institute of Pathological Anatomy (Dischka Győző Street), there were rimmed roof tiles at a D. of $170 \mathrm{~cm}$ (the $W$ part of the trench). Among the bricks remains of a child's skeleton could be identified. In the same place a small bronze ring, fragments of vessels, and of a glass vessel, and a 4thcentury burnt PB were found (AE 3, 4th century, not identifiable).

Grave R/321 (Suppl. 2). In 1969, at the N part of the courtyard of the Post Office, near Dischka Győző Street, we found the remains of a child's grave at a $\mathbf{D}$. of $270 \mathrm{~cm}$, among Roman buildings. It was disturbed, and a child's skull was found inside.

Associated articles:

1. Pottery beaker. Grey, pear-shaped. H: 12.5, DM: $8.5, \mathrm{DB}: 5 \mathrm{~cm}$. 70.9 .1 (Pl. 40, No. 6).

2. A string of beads. 115 items. Small rounded and prismatic beads, light blue in colour and made of glass paste; also one green and two made of goldcoloured glass. 70.9.2 (Pl. 40, No. 4).

3. Iron nait. With round head. L: 9, W: 0.7-1.5, Diam. of head: $2.5 \mathrm{~cm}$. 70.9 .3 ( $P l .40$, No. 5 ).
${ }^{241}$ Our attention was called to the graves by Valéria Kováts, for which we thank her here.

${ }^{242}$ Cf. Fülep: Neuere Ausgrabungen in der römischen
Stadt Sopianae (Pécs). RF Ser. II, No. 16 (Budapest 1974).

${ }^{243}$ K. Sz. Póczy: Bud. Rég. XXI (1964) p. 69. 


\section{ARTICLES FROM UNSPECIFIED SITES}

1. Gold chain. Made of 23 loops, with 16 small light blue and whitish glass paste beads. The hook and loop fastening was made of thin wire. L: $21.7 \mathrm{~cm}$. L. of loop: 1.8, W: 0.3, L. of hook: 2.5 , W: $0.6 \mathrm{~cm}$. Weight (with the stones): $3.84 \mathrm{gr}$. HNM 108/1912.9. - Coll. H.244 (Pl. 37, No. 8.)

2. A pair of gold earrings. Ruby-red glass paste in an oval setting, made of gold wire in the form of a flower with eight petals. The stem is " $\mathrm{S}$ "shaped. L: 2.5 and 2.7, respectively; Diam. of head: $1 \mathrm{~cm}$. Weight: 1.55 and $1.56 \mathrm{gr}$, respectively. HNM 108/1912.10-11. Coll. H. (Pl. 37, Nos 3a-b).

3. Remains of gold cloth. Remains of gold thread. HNM 108/1912.12. Coll. H.

4. Bronze key with ring. The ring is made of cylindrical wire, with a short stem. The ward of the key is rectangular with two teeth. Intact, it may have belonged to a jewel casket. L: 3, Diam. of ring: 2.1, Th: $0.3 \mathrm{~cm}$. HNM 108/1912.13. Coll. H.

5. Bronze earpick, fragments. HNM 108/1912. Coll. H. M.

6. Gold chain. Pairs of rather large gold rings are linked with loop of gold wire, and between them there are pairs of rings made of glass paste (29 items). With a hook and loop fastener of lyre shape. Present L: $29.5 \mathrm{~cm}$. L. of hook: $3.2, \mathrm{~W}: 1 \mathrm{~cm}$. L. of loop: $2.5, \mathrm{~W}: 1.2 \mathrm{~cm}$. Weight: $12.53 \mathrm{gr}$. HNM 108/1912.16. Coll. H. (Pl. 37, No. 9).

7. Gold chain. Made of small loops, between which bluish glass beads are stringed (some of them have fallen out, 40 are extant). Present L: $56.5 \mathrm{~cm}$. Weight: 3.75 gr. HNM 108/1912.17. Coll. H. (Pl. 37, No. 7).

8. Gold cases. Six items. Of thin glass sheet, bent into cylindrical shape, closed with a small round plate on one end. One has a tab; the others are pierced in two places in order to be stringed. They are dilapidated and worn. Inside all of them there is some paste. (Two coins: Numerian and Diocletian AE were found with them. ${ }^{245}$ ) L: 1.5-1.8, Diam: $0.7 \mathrm{~cm}$. Weight: 4.30 gr. HNM 108/1912. 18-21. Coll. H.

9. A pair of gold earrings. Rings formed of thin gold wire, the ends join in loops. On each ring there is a pendant made of fluted gold wire from the end of which the stone is missing. $\mathrm{L}$ (with pendant): 3, W: $1.5 \mathrm{~cm}$. Weight: 0.91 and $0.98 \mathrm{gr}$. HNM 108/1912.22-23. Coll. H. (Pl. 37, Nos 2a-b).

10. A pair of gold earrings. The centre part is crescent shaped with a polished, polygonal green glass bead strung on gold wire in the centre (one of the stones is smaller). Above the crescent there is a volute ornament with three small balls and three hooks above, turned back (one is missing on both pieces). On the reverse of the crescent a hook of gold wire is fastened to it. L: $1.2, W: 1.2 \mathrm{~cm}$. Weight: 2.45 and 1.27 gr. HNM 108/1912.24-25. Coll. H. (Pl. 3\%, Nos 1a-b).

11. Gold ring. Small, ribbed. Made of strong wire. The bezel is framed by a twisted wire, and the stone is missing. The bezel joins the ring with two small balls on each side. Diam.: 1.5, Th. of wire: 0.15 , L. of head: $0.8 \mathrm{~cm}$. Weight $2.07 \mathrm{gr}$. HNM 108/1912.27. Coll. H. (Pl. 37, No. 6).

12. Gold ring. A plain, narrow ring. With notched ornamentation on both edges. Diam.: 1.9, W: $0.15 \mathrm{~cm}$. Weight: 0.69 gr. HNM 108/1912.28. Coll. H. (Pl. 37, No. 4).

13. Coins. From the first part of the 3rd century. HNM 108/1912.29-30. M. Coll. H.

14. Fragment of bone statuette (head of stylus?). Naked female (?) figure, arms folded on her bosom. The head was missing. It was broken at the leg and repaired by bronze plates nailed to it at the front and back. HNM 108/1912.31. M. Coll. H.
${ }^{244}$ It was obtained by the HNM from the collection of Antal Horváth, Pécs.
${ }^{245}$ The coins registered in the Inventory of the HNM are missing. 


\section{ABBREVIATIONS}

\section{ABBREVIATIONS I}

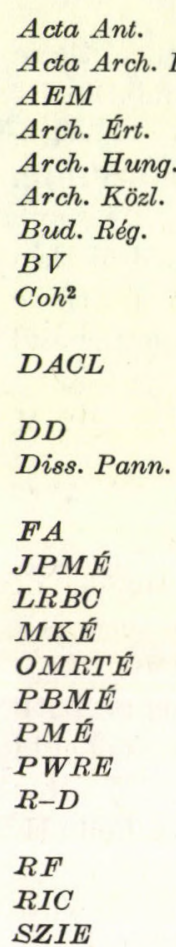

Acta Antiqua Academiae Scientiarum Hungaricae, Budapest

Acta Archaeologica Academiae Scientiarum Hungaricae, Budapest

Archaeologisch-Epigraphische Mitteilungen, Wien

Archaeologiai Értesítő, Budapest

Archaeologia Hungarica

Archaeologiai Közlemények

Budapest Régiségei

Bayerische Vorgeschichtsblätter, München

H. Cohen: Description historique des monnaies frappées sous l'empire romain communément appellées médailles impériales, Leipzig 1930.

F. Cabrol-H. Leclercq: Dictionnaire d'Archéologie Chrétienne et de Liturgie, Paris 1924-1953.

Dunántúli Dolgozatok (Papers from Transdanubia), Pécs 1974.

Dissertationes Pannonicae ex Instituto Numismatico et Archaeologico Universitatis Budapestinensis provenientes, Budapest

Folia Archaeologica, Budapest

Janus Pannonius Múzeum Évkönyve, Pécs

R. A. Garson-J. P. C. Kent: Late Roman Bronze Coinage, London 1960.

Múzeumi és Könyvtári Értesítő, Budapest

Országos Magyar Régészeti Társulat Évkönyve, Budapest

Pécs-Baranya megyei Múzeum Egyesület Értesítője, Pécs

Pécs város Majorossy Imre Múzeumának Értesítője, Pécs

Pauly-Wissowa: Realencyclopädie der klassischen Altertumswissenschaft

F. Rómer-E. Desjardins: A Magyar Nemzeti Múzeum római felíratos emlékei (The Roman Finds with Inscription of the Hungarian National Museum), Budapest 1873. Régészeti Füzetek (Magyar Nemzeti Múzeum), Budapest

H. Mattingly - M. A. Sydenham: Roman Imperial Coinage. London

Szent István Emlékkönyv (St. Stephen Commemorative Volume) 1938, Budapest

\section{ABBREVIATIONS II}

A. $\mathbf{r}$.

Acquisitions register (Museum of Pécs)

Coll. H. From the collection of Dr. Antal Horváth (Pécs)

HNM Hungarian National Museum

JPM Janus Pannonius Museum (Pécs)

NCM National Committee of Monuments 
ABBREVIATIONS USED IN THE DESCRIPTION OF GRAVES

D. Depth

H. Height

L. Length

L. S. Length of skeleton

W. Width

$\wedge \quad$ Gabled roof made of brick

\section{ABBREVIATIONS USED IN THE DESCRIPTION OF ARTICLES}

DB. Diameter of bottom

Diam. Diameter

DM. Diameter of mouth

E. Eliminated

H. Height

Inv. No. Inventory No.

L. Length

M. Missing

NI. Not identifiable

Th. Thickness

W. Width 


\section{CHANGED STREET NAMES IN PÉCS}

Present name:

Bajesy-Zsilinszky Road Bem József Street

Doktor Sándor Street

Felszabadulás Road

Geisler Eta Street

Hunyadi János Street

István Square

Janus Pannonius Street

Jókai Square

Kossuth Lajos Square

Kossuth Lajos Street

Kulich Gyula Street

Leonardo da Vinci Street

Mecsekalja-Ưttörő Road

Nagy Jenő Street

Rózsa Ferene Street

Rudas László Street

Sallai Street

Szalai András Street

Székesfehérvár Street
Name before 1945:

Siklósi Road

Irgalmasok Street

Sziget Highway

Mohács Highway

Apáca Street

Inczédy Dénes Street

Scitovszky Square

Kardos Kálmán Street

Kis Square

Majláth Square

Király Street

Papnövelde Street

Szepessy Street

Alsó-Makár Road

Kaszárnya St.

Alsó-Malom Street

Gründler Street

Ferencesek Street

Regős Street

Vörösmarty Street 
Baranya II

Benkö

Burger I

Burger II

Doppelfeld I

Doppelfeld II

Fejes

Fülep I

Fülep II

Fülep III

Fülep IV

Fülep V

Gosztonyi I

Gosztonyi II

Gosztonyi III

Gosztonyi IV

Haas

Intercisa II

Isings

Kádár I

Kádár II

Keller

Kisa

Koller

Köemlékek (Stone Finds)

Kuzsinszky

Martin

Nagy

Patek

Radnóti I

Radnóti II

Szőnyi I
B. Posta: Baranya vármegye története az őskortól a honfoglalásig. Baranya múltja és jelenje. II (The History of Baranya County from the Prehistoric Age till the Hungarian Occupation. The Past and Present of Baranya). Ed. by F. Várady. Pécs 1897.

A. Benkő: Üvegcorpus (Glass Corpus) RF Ser. II, No. 11. Budapest 1962.

A. Sz. Burger: The Late Roman Cemetery at Ságvár, Acta Arch. Hung. 18 (1966).

A. Sz. Burger: Rómaikori temető Majson (Roman Cementery at Majs), Arch. Ért. 99 (1972).

O. Doppelfeld: Das Kölner Diatretglas und die andere Netzdiatrete. Gymnasium 68. Köln 1961.

O. Doppelfeld: Römisches und fränkisches Glas in Köln, Köln 1966.

Gy. Fejes: Római pénzlelet Pécs város csatornázásánál (Roman Coins from the Drainage Works in Pécs), PBMÉ 11 (1930).

F. Fülep: Újabb kutatások a pécsi későrómai temetőben (Recent Excavations in the Late Roman Cementery of Pécs). Arch. Ért. 89 (1962).

F. Fülep: Későrómai temető Pécs-Geisler Eta u. 8. sz. alatt (Late Roman Cemetery at 8, Geisler Eta Street, Pécs), Arch. Ért. 96 (1969).

F. Fülep: Scavi archeologici a Sopianae. Corsi di cultura sull' arte Ravennate e Bizantina, Faenza 1969.

F. Fülep-A. Fetter: Neuere Forschungen in der ausgemalten frühchristlichen Grabkammer No. II von Pécs. JPMÉ 14-15 (Pécs 1969-1970).

F. Fülep: Sopianae (in print).

Gy. Gosztonyi: A pécsi Szent Péter székesegyház eredete (The Origin of St. Peter's Cathedral at Pécs), Pécs 1939.

Gy. Gosztonyi: A pécsi hétkaréjos temetői épület (The Cella Septichora in the Cemetery of Pécs), Arch. Ért. (1940).

Gy. Gosztonyi: A pécsi II. számú ókeresztény sírkamra és sírkápolna (The Painted Early Christian Tomb Chamber II and Chapel of Pécs), Arch. Ért. (1942).

Gy. Gosztonyi: A pécsi ókeresztény temetö (The Early Christian Cemetery of Pécs), Pécs 1943. M. Haas: Gedenkbuch der $K$. freien Stadt Fünfkirchen, Pécs 1852.

Dunapentele. Geschichte der Stadt in der Römerzeit. Arch. Hung. XXXVI, Budapest 1957.

G. Isings: Roman Glass from Dated Finds, Groningen 1957.

Z. Kádár: Pannónia ókeresztény emlékeinek ikonográfiája (The Iconography of the Early Christian Finds from Pannonia), Regnum 3. (1938-1939).

Z. Kádár: A triumphus-eszme a pécsi ókeresztény héroon egyik freskóján (The Idea of Triumph on one of the Wall Paintings of the Early Christian Heroon) Regnum 4. (1940-1941).

E. Keller: Die spätrömischen Grabfunde in Südbayern, München 1971.

A. Kisa: Die antiken Glaser der Frau Maria v. Rath zu Köln, Bonn 1899.

J. Koller: Prolegomena in historiam episcopatus Quinqueeclesiarum, Posonii 1804.

F. Fülep-A. Sz. Burger: Pécs rómaikori kőemlékei (The Roman Age Stone Finds from Pécs). DD 7 (Pécs 1973).

B. Kuzsinszky: A Balaton környékének archaeológiája (The Archaeology of the Balaton Region), Budapest 1920.

M. Martin: Zwei spätrömischen Gürtel aus Augst/BL. Römerhaus und Museum Augst. Jahresbericht 1967, Augst 1968.

L. Nagy: Pannonia Sacra, SZIE, Budapest 1938.

E. Patek: A pannóniai fibulatípusok elterjedése és eredete (The Origin and Distribution of the Pannonian Types of Brooches). Diss. Pann. Ser. II, No. 19, Budapest 1942.

A. Radnóti: A pannóniai római bronzedények (Roman Bronz Vessels from Pannonia). Diss. Pann. Ser. II, No. 6, Budapest 1938.

A. Radnóti: Spätrömische Gräber aus Burgheim, $B V$, Heft 23, München 1958.

O. Szőnyi: A pécsi őskeresztény sírkamra (The Early Christian Tomb Chamber at Pécs), Budapest 1907. 
Szönyi II

Török I

Török II
O. Szőnyi: Ásatások a pécsi székesegyház környékén 1922-ben (Excavations in the Vicinity of the Cathedral of Pécs in 1922), OMRTÉ II, Budapest 1923-26.

Gy. Török: A pécsi belvárosi templom bővítésénél előkerült római leletek (Roman Finds found during the Enlargement of the City Parish Church of Pécs), FA II-IV, Budapest 1941.

Gy. Török: Rómaikori sírkamrák Pécs felső-sétaterén (Roman Age Tomb Chambers from the Upper Promenade of Pécs). Arch. Ért. (1942). 


\section{INDEX}

Graves

Burial-chamber I, with wall paintings Burial-chamber II, with wall paintings Burial-chamber III

Burial-chamber IV

Burial-chamber V

Burial-chamber VI

Burial-chamber VII

Burial-chambers VIII-IX

Burial-chamber $\mathrm{X}$

Burial-chamber XI

Burial-chamber XII (?)

Cella trichora

Cella septichora

$\mathrm{R} / \mathbf{1 - 1 3}$

$\mathrm{R} / 14-18$

$\mathrm{R} / 19$

$\mathrm{R} / 20-22$

$\mathrm{R} / 23-24$

$\mathrm{R} / 25-29$

$\mathrm{R} / 30-31$

$\mathrm{R} / 32$

$\mathrm{R} / 33-35$

$\mathrm{R} / 36-39$

$\mathrm{R} / 40$

Undercroft of Cathedral, Graves A/I-III

$\mathrm{R} / 41$

István Square

$\mathrm{R} / 42-43$

$\mathrm{R} / 44-46$

$\mathrm{R} / 47-50$

$\mathrm{R} / 51-53$

$\mathrm{R} / 54$ (?)

$\mathrm{R} / 55-91$

$\mathrm{R} / 92-105$

R/106-107

$\mathrm{R} / 108$

$\mathrm{R} / 109-111$

$\mathrm{R} / 112$

$\mathrm{R} / 113$

$\mathrm{R} / 114$

$\mathrm{R} / 115$

$\mathrm{R} / 116$

$\mathrm{R} / 117$

$\mathrm{R} / 118-124$

$\mathrm{R} / 125-145$

$\mathrm{R} / 146$

$\mathrm{R} / 147-149$

$\mathrm{R} / 150$

$\mathrm{R} / 151-163$

$\mathrm{R} / 164-176$

$\mathrm{R} / 177-183$

$\mathrm{R} / 184$

$\mathrm{R} / 185-189$

$\mathrm{R} / 190$

$\mathrm{R} / 191-193$
12, István Square

12, István Square

15, István Square István Square

21, István Square Janus Pannonius Street Janus Pannonius Street

3, Janus Pannonius Street

5, Janus Pannonius Street

4, Janus Pannonius Street Geisler Eta Street

4, Geisler Eta Street

6, Geisler Eta Street

6, Geisler Eta Street

7, Geisler Eta Street

8, Geisler Eta Street

10, Geisler Eta Street

14, Geisler Eta Street

23, Geisler Eta Street

23, Geisler Eta Street Geisler Eta Street Geisler Eta Street Geisler Eta Street (Tallián)

1, Székesfehérvár Street Corner of Székesfehérvár Street and Geisler Eta Street

2, Székesfehérvár Street

1, Leonardo da Vinci Street Széchenyi Square
Date of finding Page

\begin{tabular}{|c|c|c|c|c|c|}
\hline 1780 & . . & . & . & . & . 11 \\
\hline 1939 & . . & . & . & . & . 11 \\
\hline 1913 & . . & . & . & . & . 12 \\
\hline 1913 & . . & . & . & . & . 14 \\
\hline 1913 & . . & . & . & . & . 14 \\
\hline 1922 & . . & . & . & . & . 15 \\
\hline 1958 & . . & . & . & . & . 15 \\
\hline 1940 & . . & . & . & . & . 16 \\
\hline 1841 & . . & . & . & . & . 17 \\
\hline 1716- & 1726 & $?$ & . & . & . 25 \\
\hline 1927 &. & . & . & . & . 37 \\
\hline 1922 & . . & - & . & . & . 15 \\
\hline 1938- & 1940 & & . & . & . 16 \\
\hline 1780 & . . & . & . & . & . 11 \\
\hline 1913 & . . & . & . & . & . $11-14$ \\
\hline 1913 & . . & . & . & . & . 14 \\
\hline 1913 (? & & . & . & . & . 14 \\
\hline 1922 & . . & . & . & $\cdot$ & . 15 \\
\hline 1954 & . . & . & . & . & . 15 \\
\hline 1922 & . . & . & . & . & . 15 \\
\hline 1958 & . . & . & . & . & . 16 \\
\hline 1938 &. & . & . & . & . 16 \\
\hline 1941 & . . & . & . & . & . 17 \\
\hline 1964 & . . & . & . & . & . 17 \\
\hline 1970 & . . & . & . & . & . 17 \\
\hline 1927 & . . & . & . & . & . 23 \\
\hline 1927 & . . & . & . & . & . 23 \\
\hline 1931 & . . & . & . & . & . 23 \\
\hline 1926 & . . & . & . & . & . 24 \\
\hline 1937 & . . & . & . & . & . 24 \\
\hline 1842 & (?) & . & . & . & . 24 \\
\hline 1716 & -1726 & & . & . & . 24 \\
\hline 1934 & -193 & & . & . & . 25-26 \\
\hline 1927 & . . & . & . & . & . 26 \\
\hline 1927 & .. & . & . & . & . 26 \\
\hline 1927 & . . & . & . & • & . 27 \\
\hline & . . & . & . & . & . 27 \\
\hline 1914 & . . & . & . & . & . 27 \\
\hline & . . & . & . & . & . 27 \\
\hline 1938 & . . & $\cdot$ & . & ${ }^{\circ}$ & . $27-28$ \\
\hline 1927 & . . & . & . & . & . 28 \\
\hline 1926 & . . & . & . & - & . 28 \\
\hline 1934 & . . & • & . & . & . 28 \\
\hline 1906 & . . & . & . & . & . 28 \\
\hline 1870 & . & $\cdot$ & . & - & . $28-29$ \\
\hline 1927 & . & & . & . & . 29 \\
\hline 1927 & . & . & . & - & . 29 \\
\hline 1935 & . & $\cdot$ & . & - & . $29-30$ \\
\hline 1910 & . . & ${ }^{\circ}$ & . & * & . $30-32$ \\
\hline 1927 & . . & & . & & . $\quad 32-34$ \\
\hline 1927 & . & & . & • & . $34-35$ \\
\hline 1959 & . & & . & & . 35 \\
\hline 1897 & . . & & . & . & . 35 \\
\hline 1897 & . . & $\cdot$ & . & . & . $36-37$ \\
\hline
\end{tabular}


Graves

R/194-195

R/196

$\mathrm{R} / 197-198$

R/199-200

$\mathrm{R} / 201$

$\mathrm{R} / 202-217$

$\mathrm{R} / 218$

$\mathrm{R} / 219-220$

$\mathrm{R} / 221$

$\mathrm{R} / 222$

$\mathrm{R} / 223$

$\mathrm{R} / 224$

$\mathrm{R} / 224 \mathrm{a}$

R/225-226

$\mathrm{R} / 227$

$\mathrm{R} / 228$

$\mathrm{R} / 229$

$\mathrm{R} / 230$

$\mathrm{R} / 231-233$

$\mathrm{R} / 233 \mathrm{a}$

R/234-245

$\mathrm{R} / 246$

$\mathrm{R} / 247$

$\mathrm{R} / \mathbf{2 4 8}$

$\mathrm{R} / 249-250$

$\mathrm{R} / 251$

$\mathrm{R} / 252$

$\mathrm{R} / 253$

$\mathrm{R} / 254$

$\mathrm{R} / 255-256$

R/257-258

$\mathrm{R} / 259$

R/260-261

$\mathrm{R} / 262$

R/263-264

$\mathrm{R} / 265$

R/266-273

$\mathrm{R} / 274$

$\mathrm{R} / 275$

$\mathrm{R} / 276$

$\mathrm{R} / 277$

R/278-279

$\mathrm{R} / 280$

R/281-289

$\mathrm{R} / 290$

R/291-292

$\mathrm{R} / 293$

$\mathrm{R} / 294$

$\mathrm{R} / 295-297$

$\mathrm{R} / 298$

R/299-300

R/301

$\mathrm{R} / 302$

$\mathrm{R} / 303$

$\mathrm{R} / 304$

$\mathrm{R} / 305$

$\mathrm{R} / 306$

R/307-308

R/309-314

R/315-317

$\mathrm{R} / 318-321$
Hunyadi János Street

5, Hunyadi János Street

12, Széchenyi Square

Széchenyi Square

Széchenyi Square

Széchenyi Square

Jókai Square

Sallai Street

1, Váradi Antal Street

7/2, Váradi Antal Street

6, Bem József Street

19, Rákóczi Road

49, Rákóczi Road

58, Rákóczi Road Bajesy-Zsilinszky Road

8, Bajesy-Zsilinszky Road Bajesy-Zsilinszky Road Bajcsy-Zsilinszky Road Bajesy-Zsilinszky Road and Majláth Street

19-21, Majláth Street

Szalai András Street

Rózsa Ferenc Street

Kossuth Lajos Square

1, Kossuth Lajos Square

14, Perczel Street

24, Perczel Street

Corner of Perczel and

Gábor Street

4, Felső-Malom Street

3, Kossuth Lajos Street

13, József Street

18-20, József Street József Street

18, Anna Street

20, Anna Street

Rudas László Street

1, Tettye Street

Customs house on

the road to Buda

Basamalom Lane

Buda Garden City (Diós Lane)

Felszabadulás Road

Felszabadulás Road

Felszabadulás Road

Zelms sand-pit

Power Station

Power Station

Tádé Sikorski's brick-yard

Brick-yard of Mrs Sikorski

3, Alajos Street

12, Attila Street

8, Árpád Street

28, Makár Street

14, Mecsekalja-Ưttörő Road

Airport

Makáralja

Makárhegy-Misetics vineyard

Makárhegy-foot of the hill

Aranyhegy

Cserkút

Doktor Sándor Street

Kiskőszeg Street

Dischka Győző Street

1927 . . . . 37

1927 . . . . 37

1963 . . . . 38

1930 . . . . 38

1959 . . . . . 38

1939 . . . . . 38-41

. . . . 41

1886 . . . . . 41

1927 . . . . 41

1927 . . . . . 42

1927 . . . . . 42

1927 . . . . 42

. . . . . 42

1955 . . . . 42-43

. . . . . 43

1900 . . . . 43

1927 . . . . 43

. . . . 44

1928 . . . . . 44

. . . . . 44

1940 . . . . . 44-45

1928 . . . . 45

1926 . . . . 45

1958 . . . . . 45

1926 . . . . 45-46

1962 . . . . . 46

1961 . . . . 46

1927 . . . . . 46

1870 . . . . . 46

1927 . . . . . 46

1927 . . . . . 46-47

1927 . . . . 47

1927 . . . . 47

1927 . . . . . 47

1941 . . . . . 48

1962 . . . . . 48

1870 . . . . . . 48-49

1948 . . . . . 49

1927 . . . . 50

1938 . . . . . 51

1941 . . . . 51

1958-1959 . . . 51-52

1880 . . . . . 52

1956 . . . . 52

1957 . . . 52

1908 . . . . . 52-53

1912 . . . . 53

. . . . . 53

1928 . . . . 53

. . . . . 53

... . . 53

1963 . . . . . 54

1956 . . . . 54

... . 54

... . . 54

1931 . . . . 54

1845 (?) . . . . 55

1907 . . . . 55

1970 . . . . 55

1970 . . . . . 56

1963-1964 . . . 56 
PLATES 


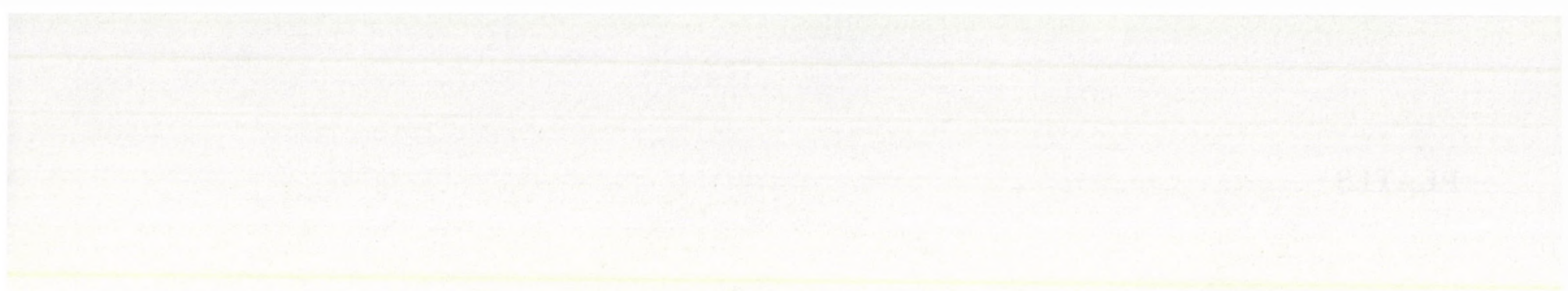



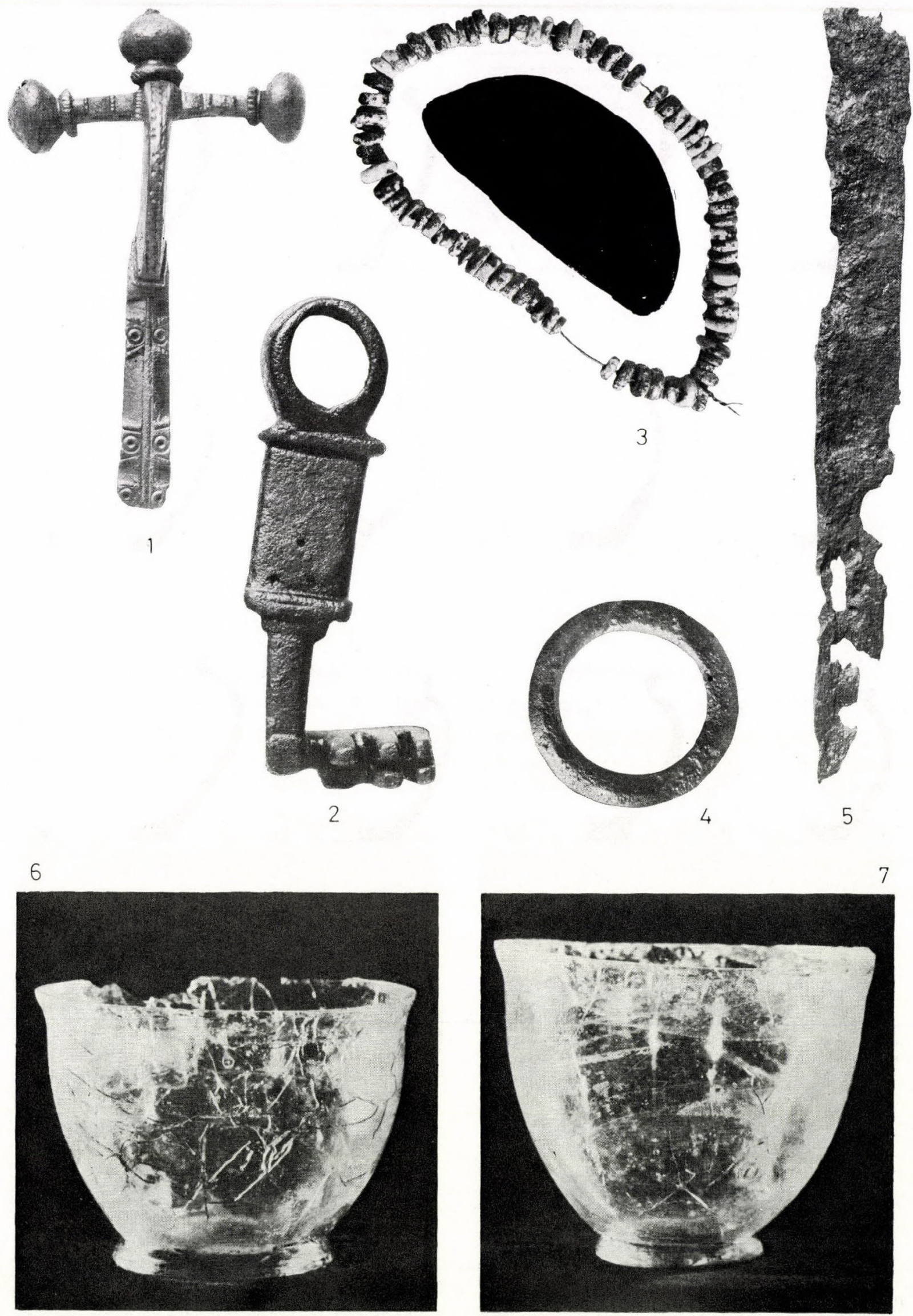

Plate 1 1, 5: Grave R/15; 2: Burial-chamber IV; 3, 4, 6, 7: Grave R/19 

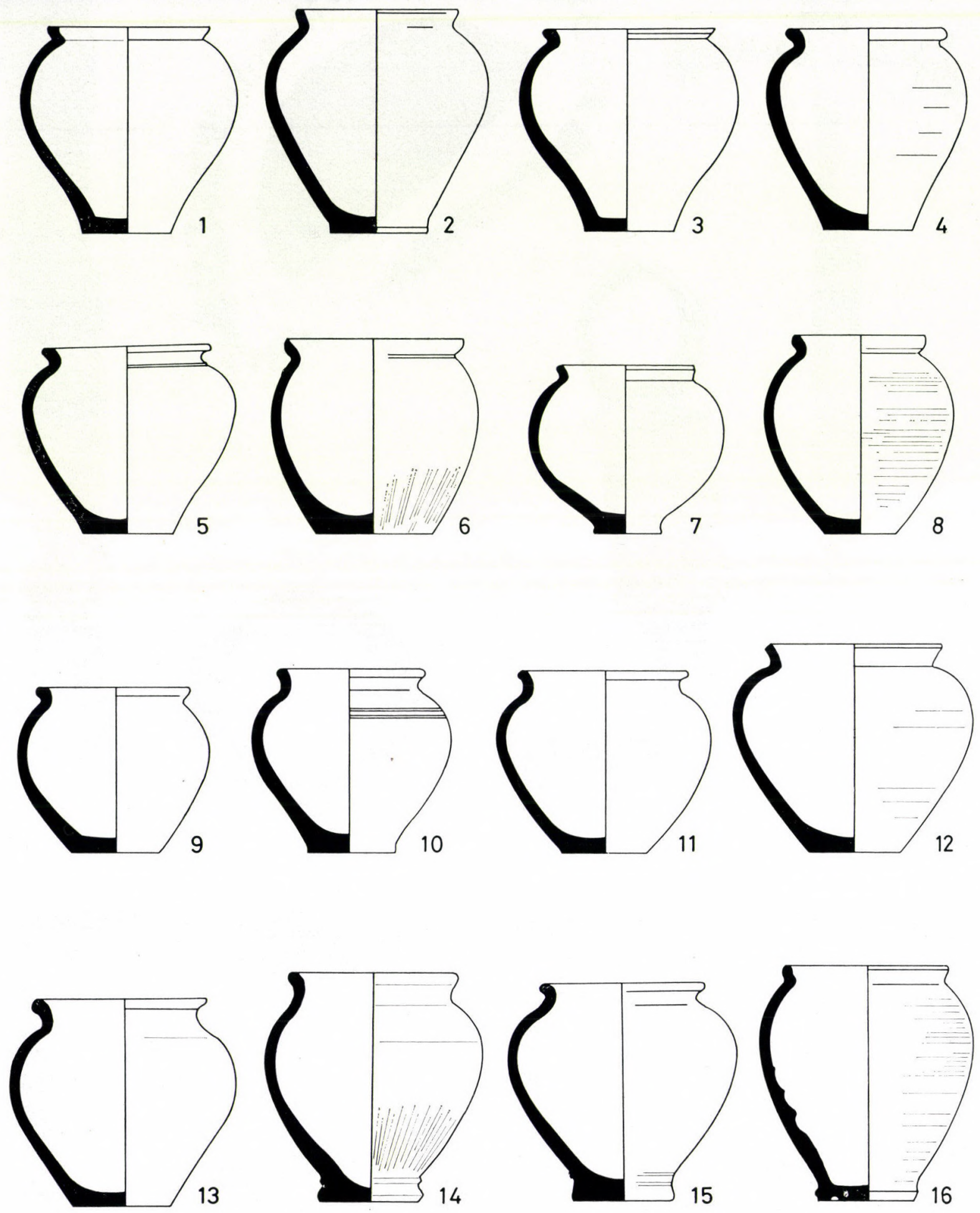

Plate 2 Grave R/18: pottery 

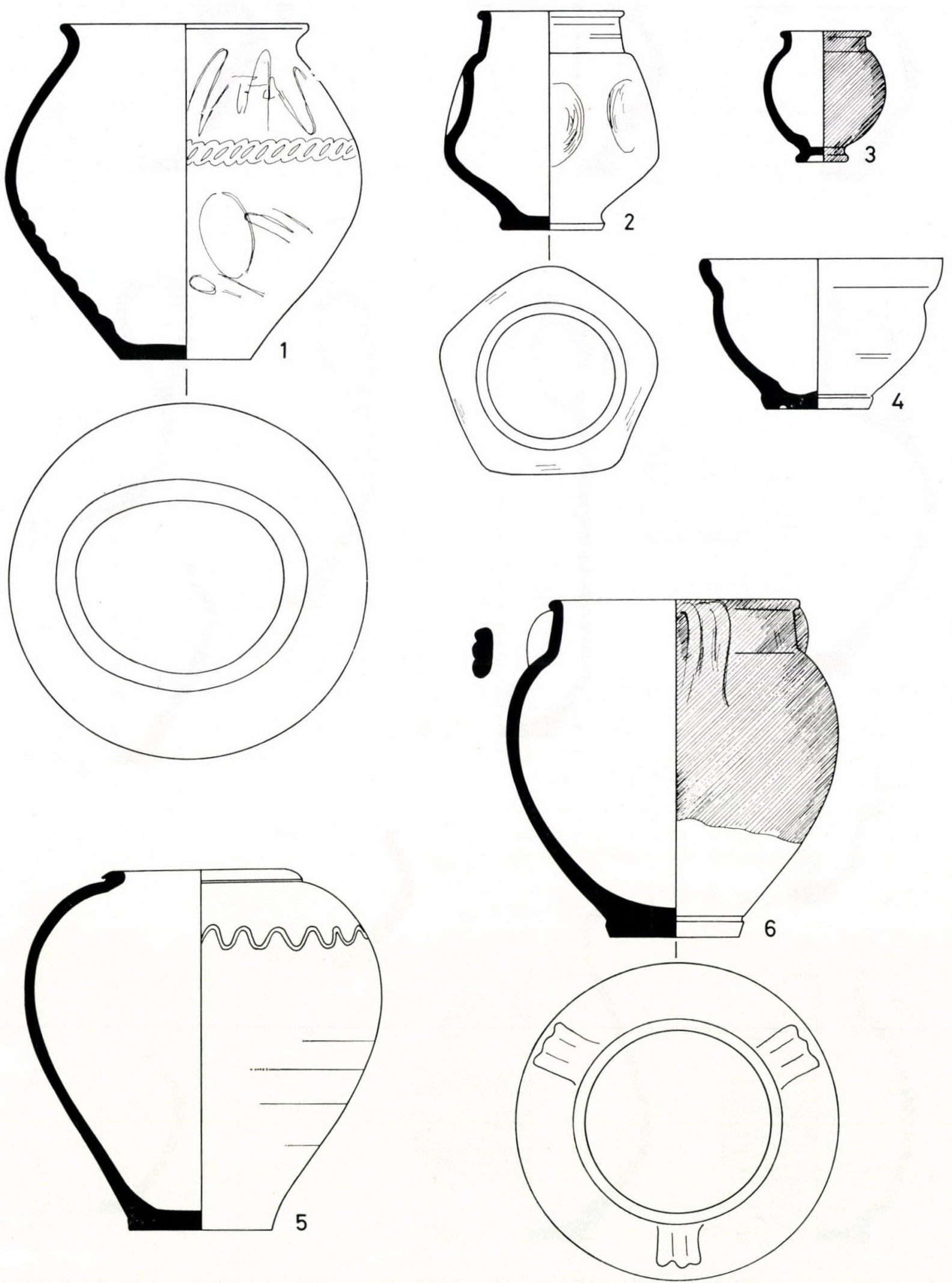

Plate 3 Grave $\mathrm{R} / 18$ : pottery 


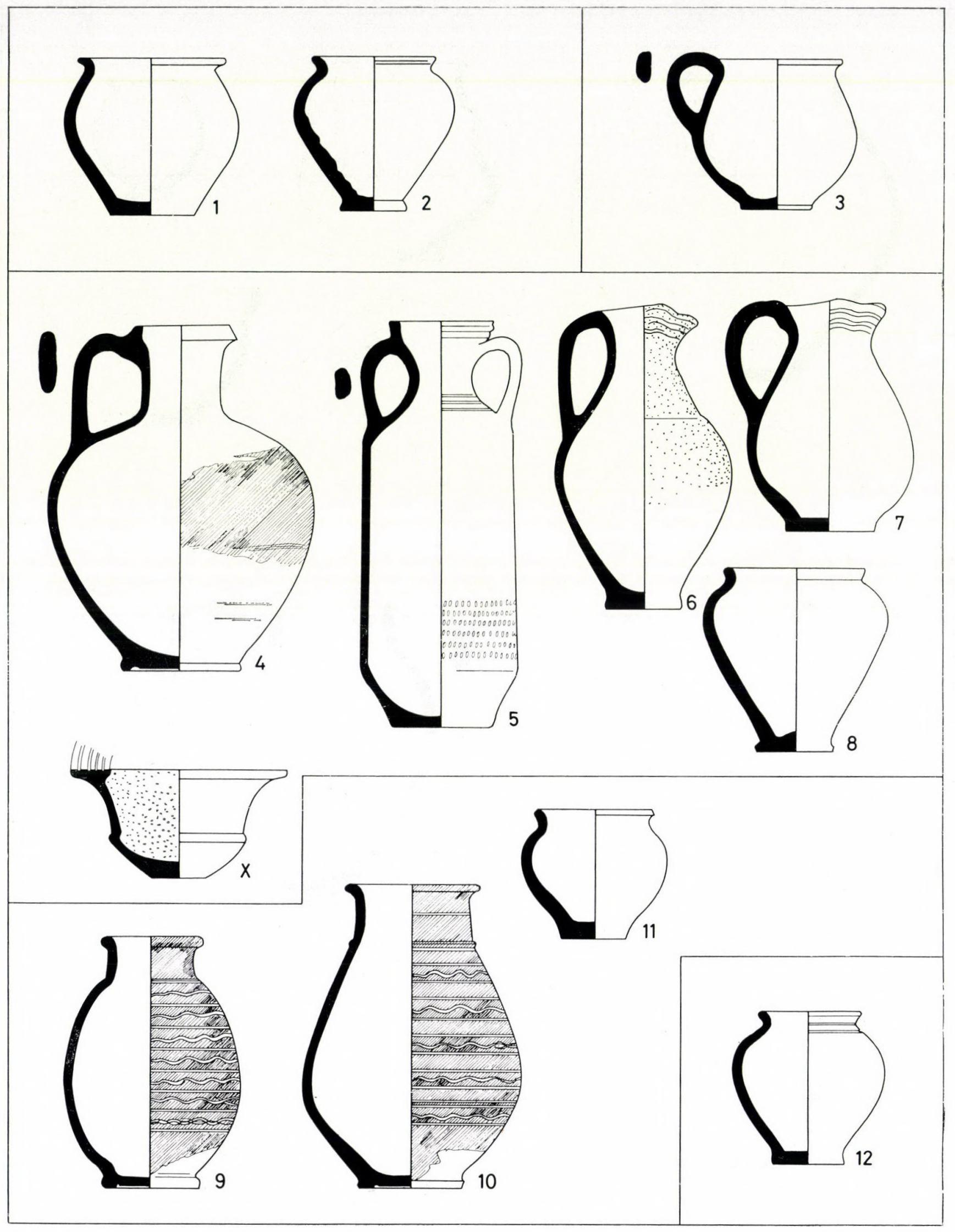

Plate 4 Grave R/18: pottery 


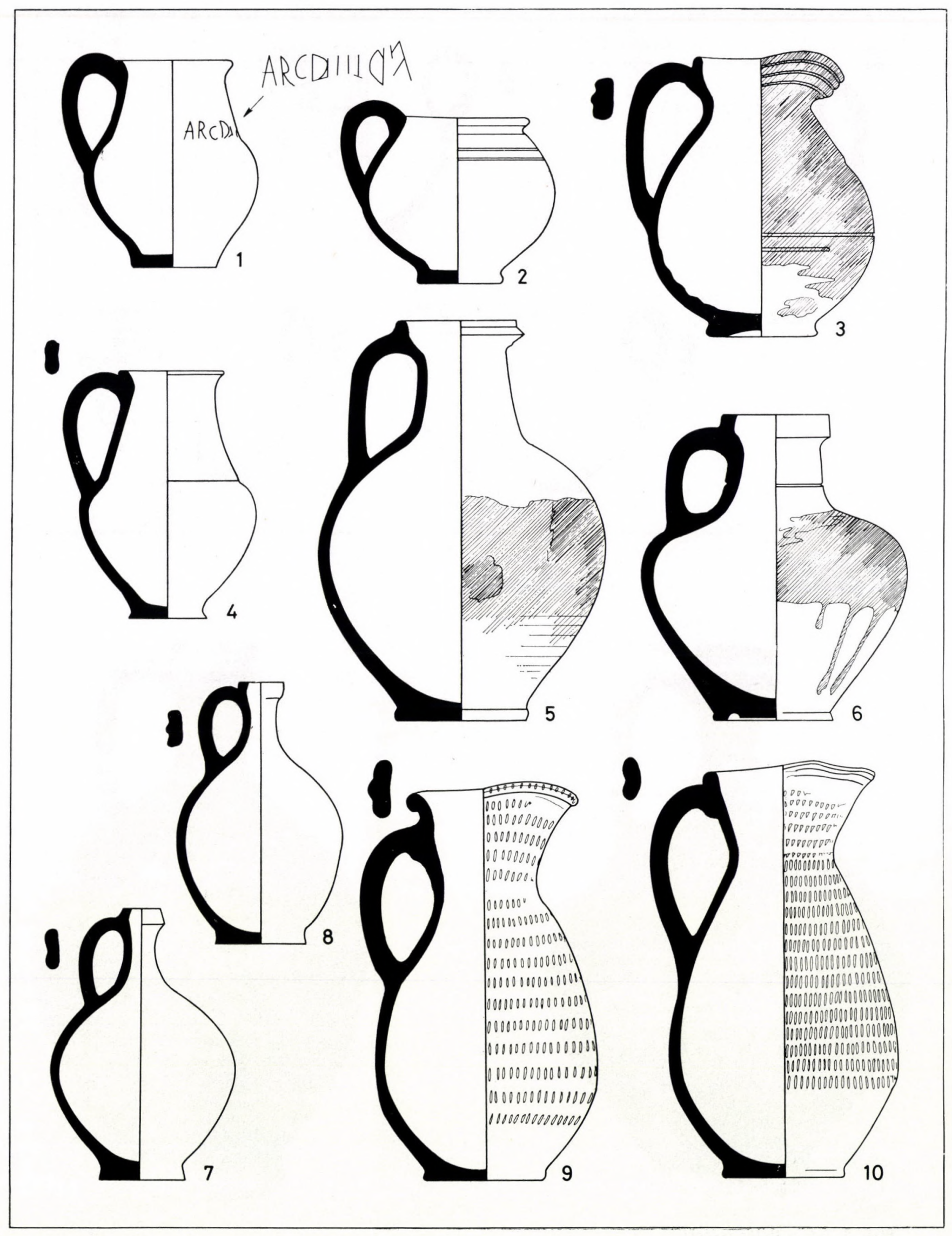

Plate 5 Grave R/18: pottery 

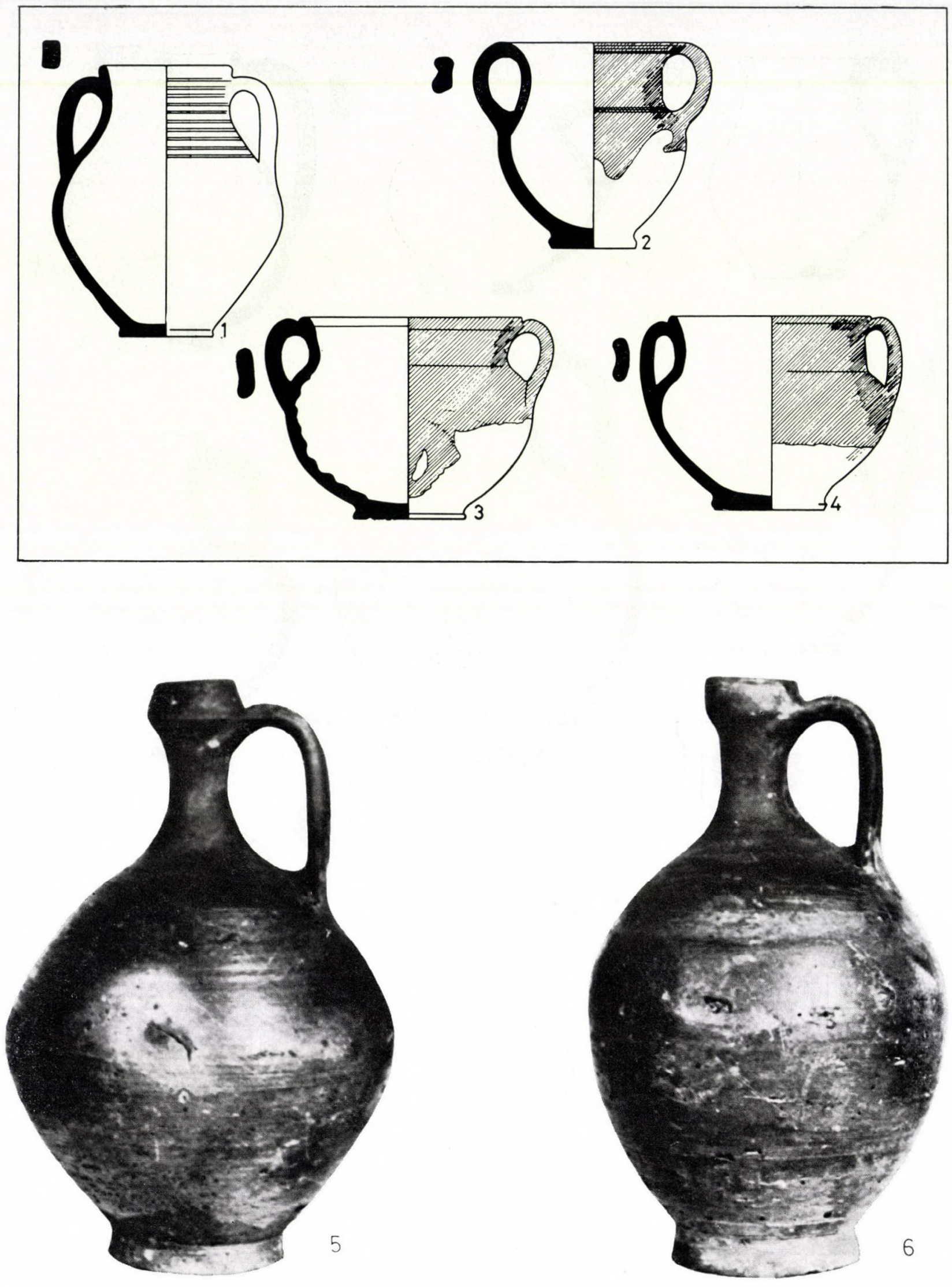

Plate 6 Grave R/18: pottery 

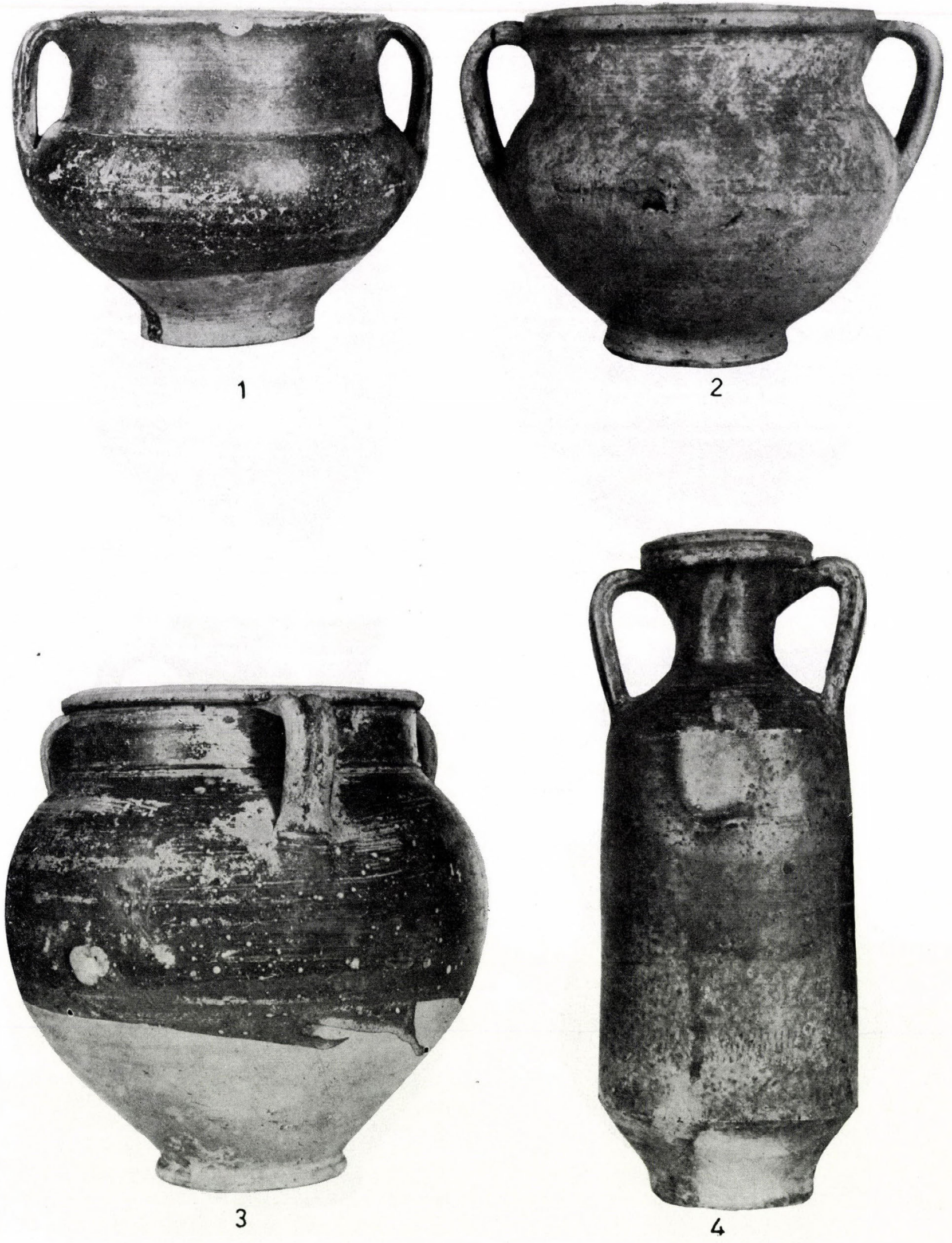

Plate 7 Grave R/18: pottery 

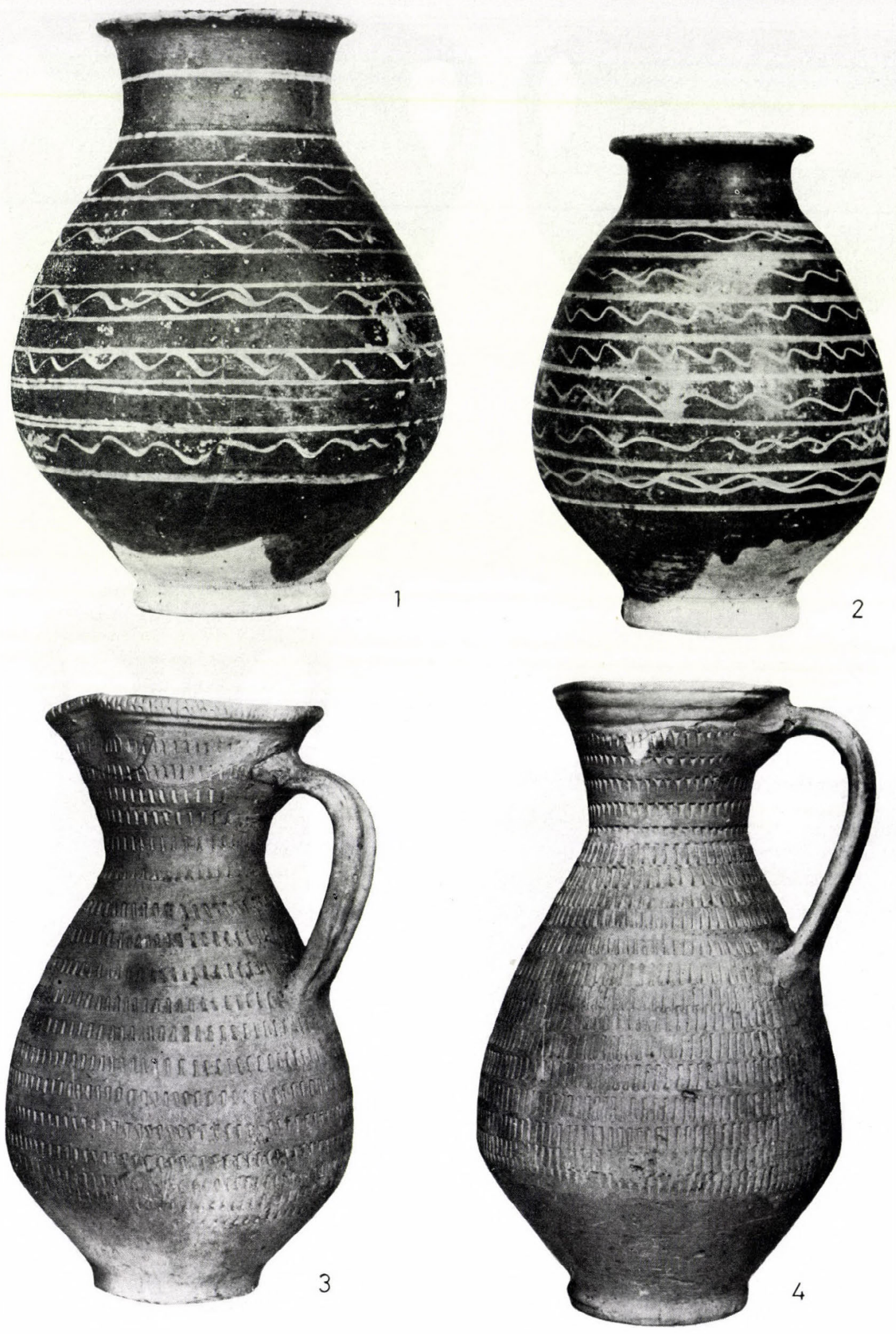

Plate 8 Grave R/18: pottery 

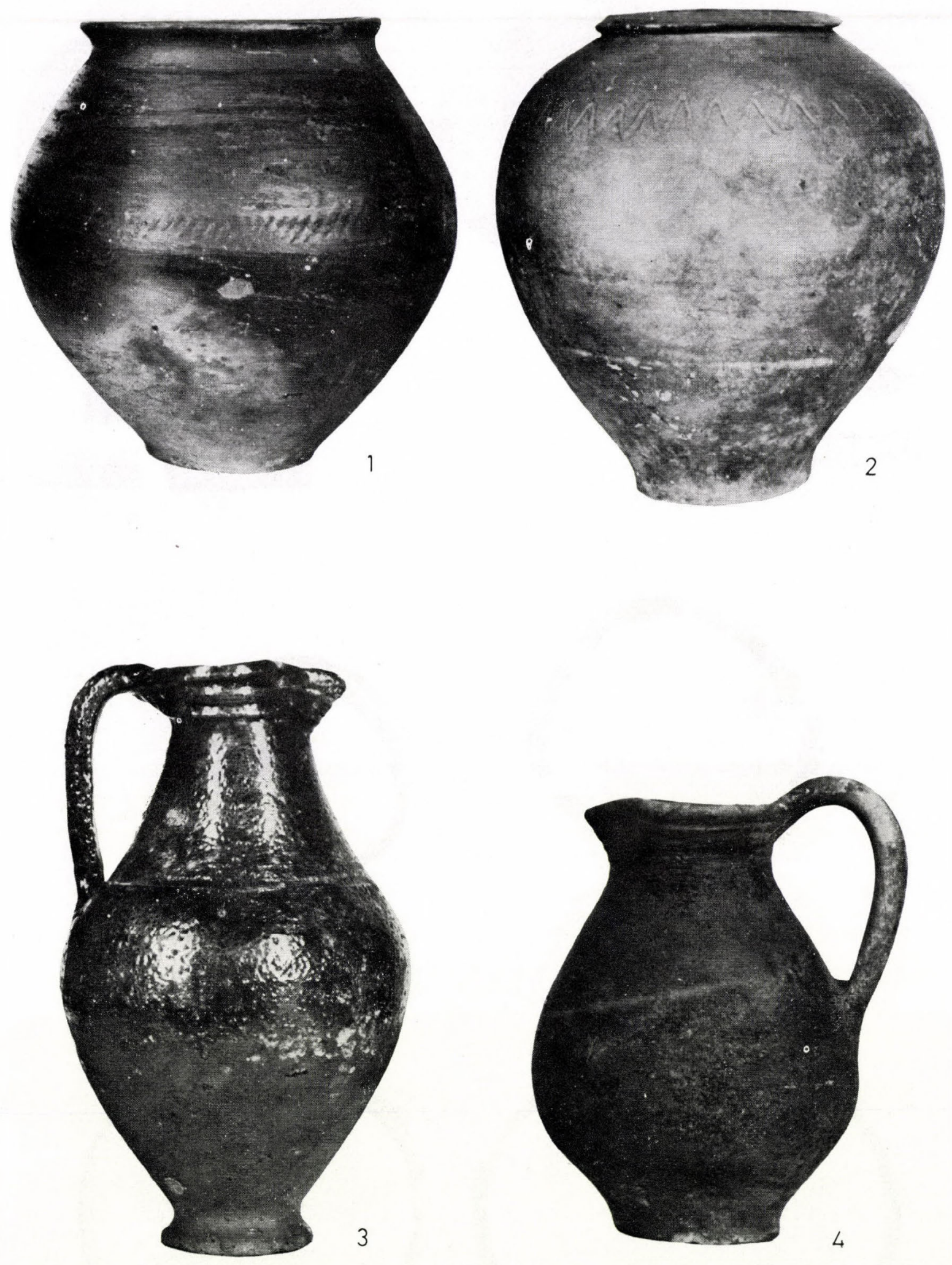

Plate 9 Grave R/18: pottery 


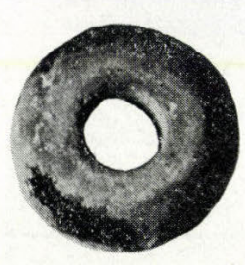

1

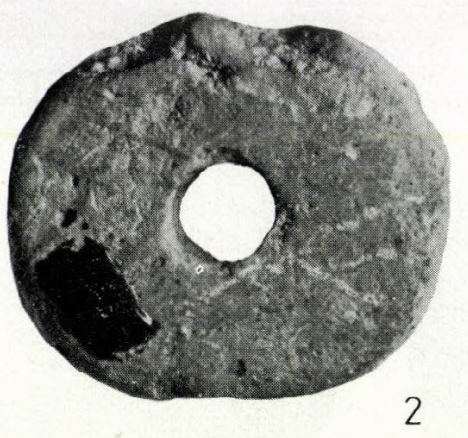

2
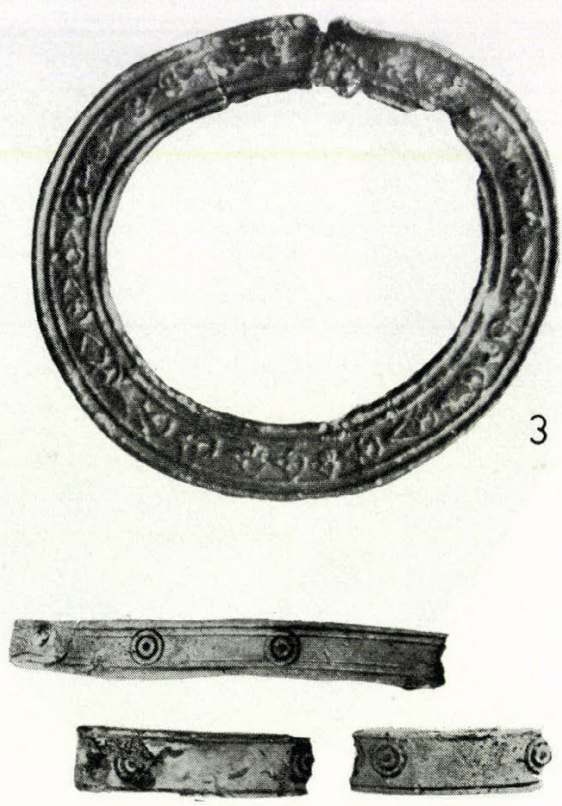

5

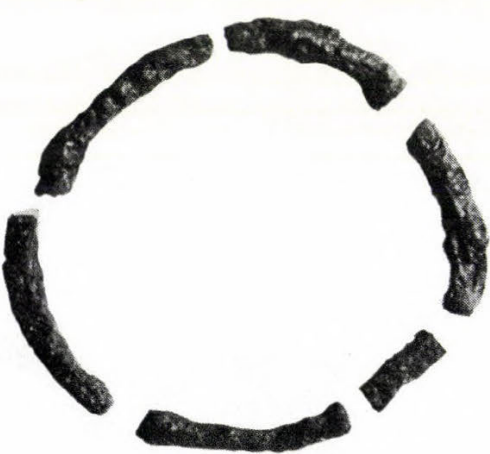

6
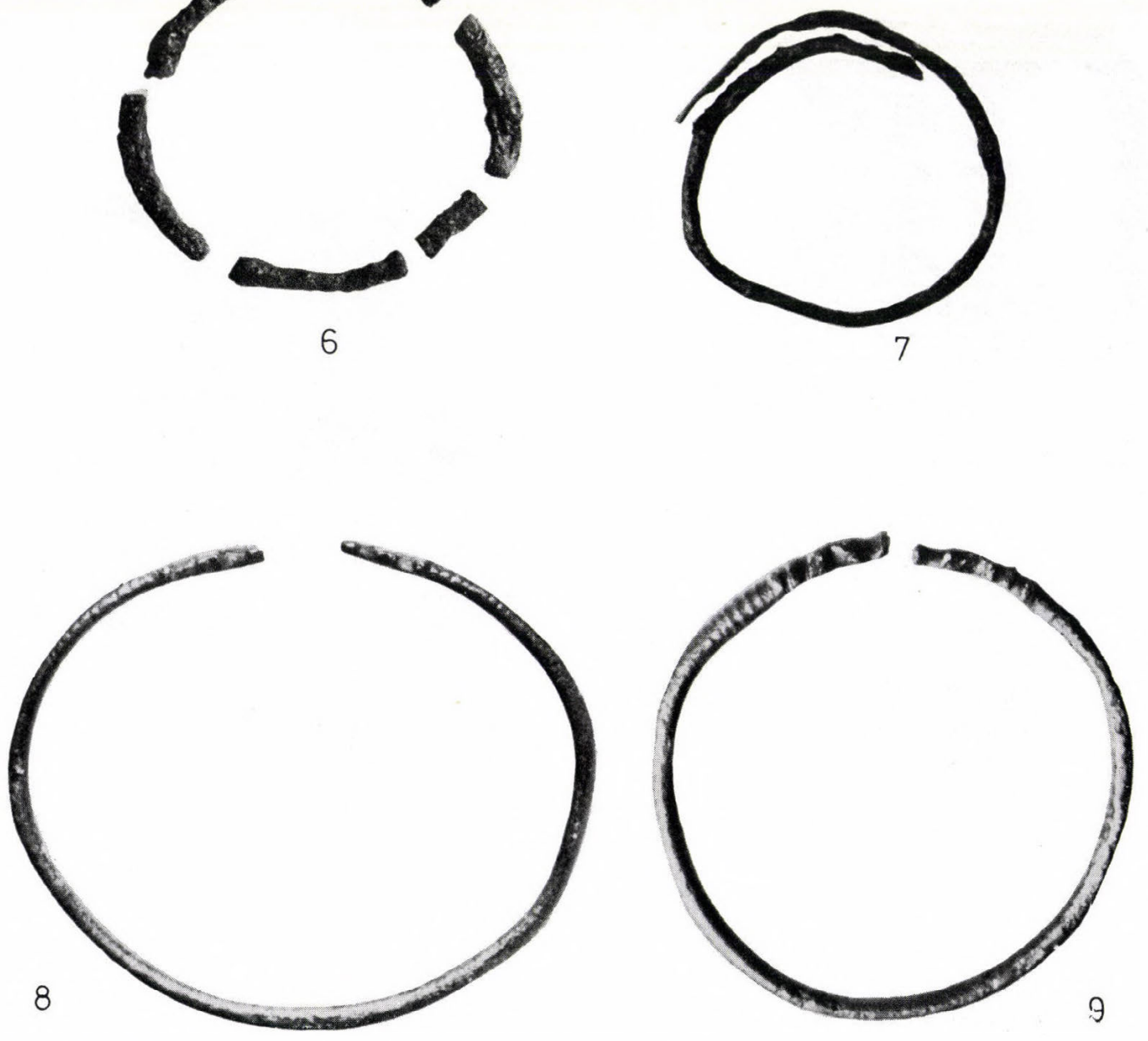

Plate 10 1-3: Grave R/33; 4-7: Grave R/34; 8-9: Upper Promenade 

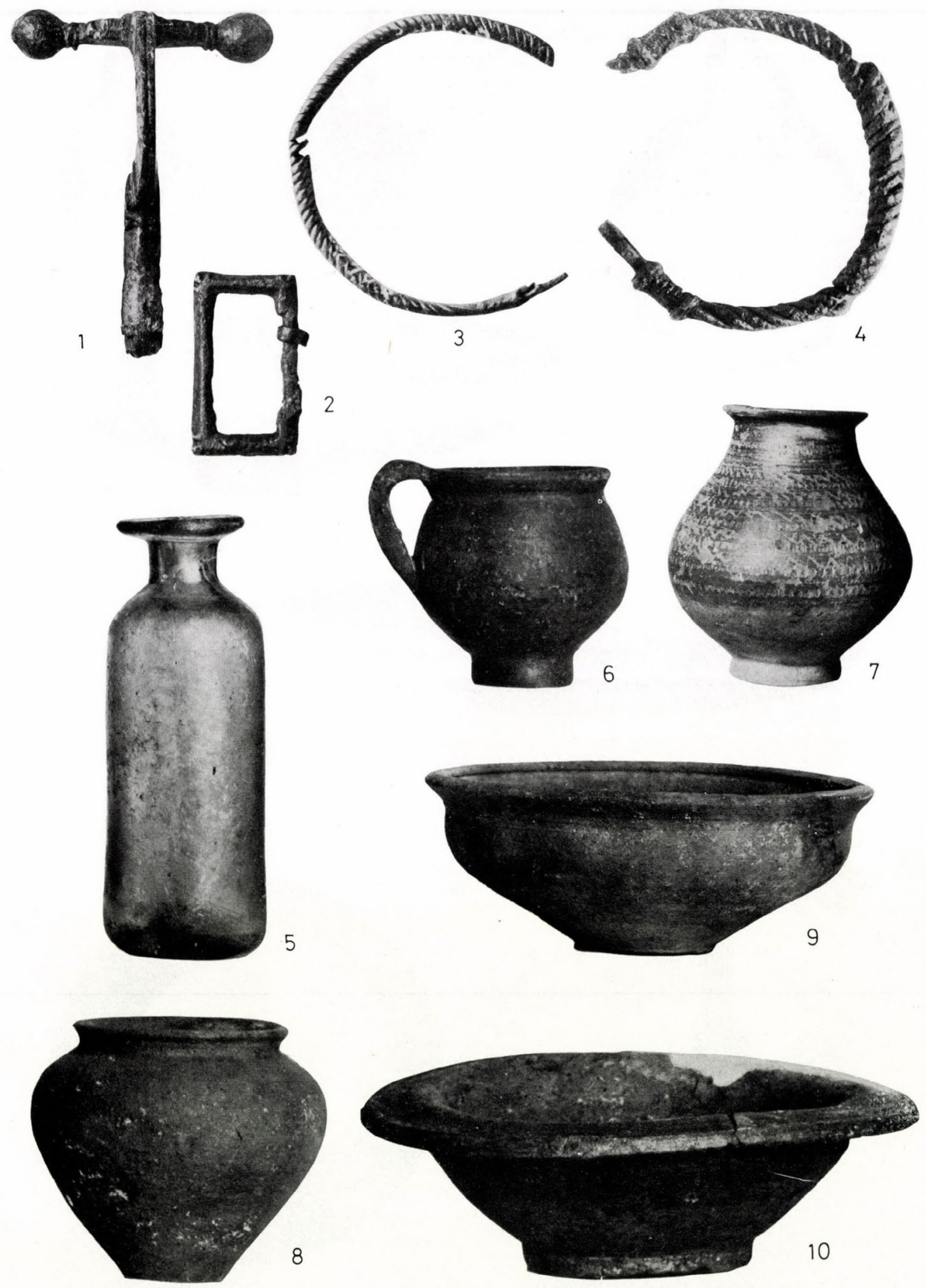

Plate 11 Graves R/92-105 

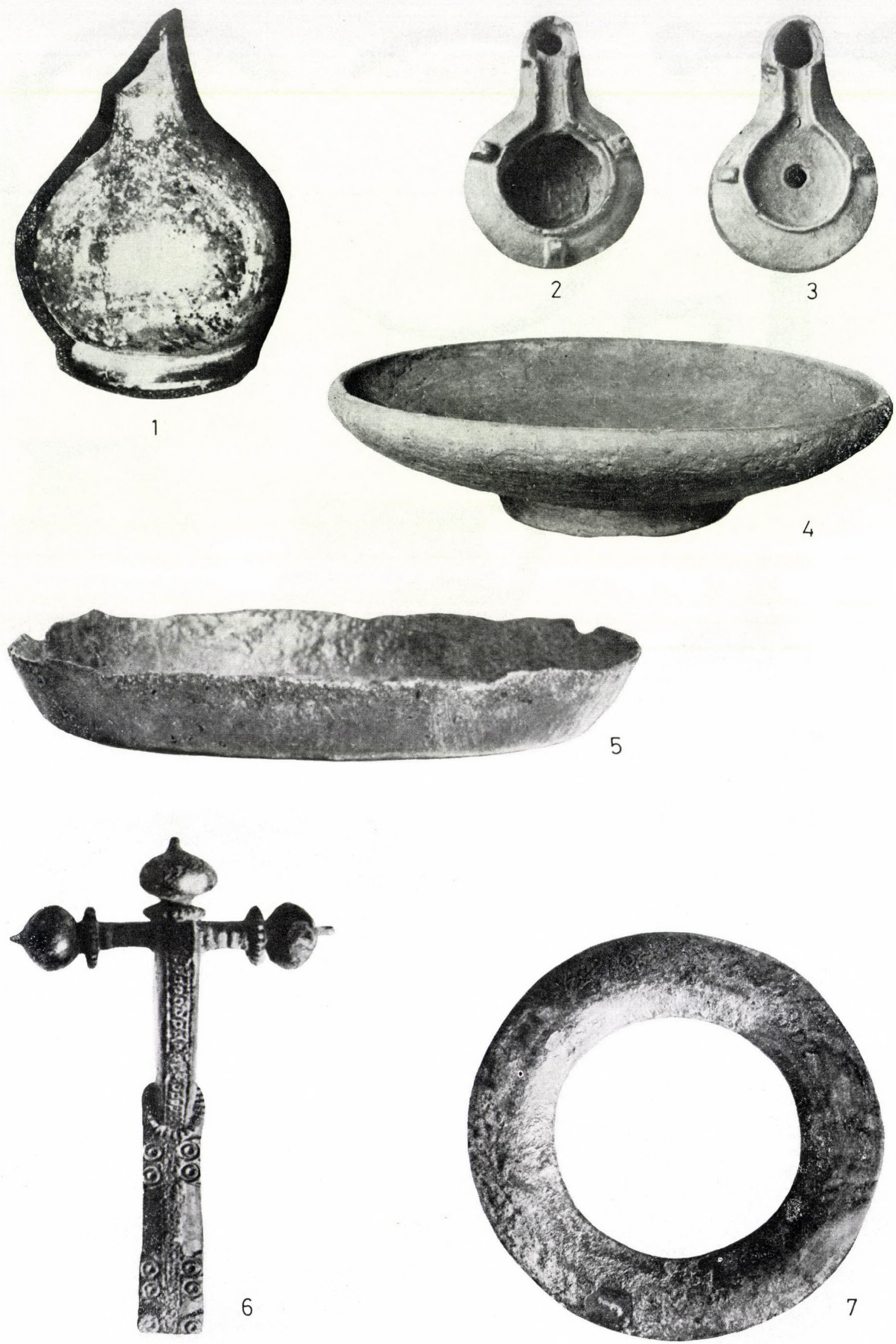

Plate 12 1: Grave R/48; 2, 3, 5: Grave R/108; 4: Grave R/109; 6, 7: Grave R/112 


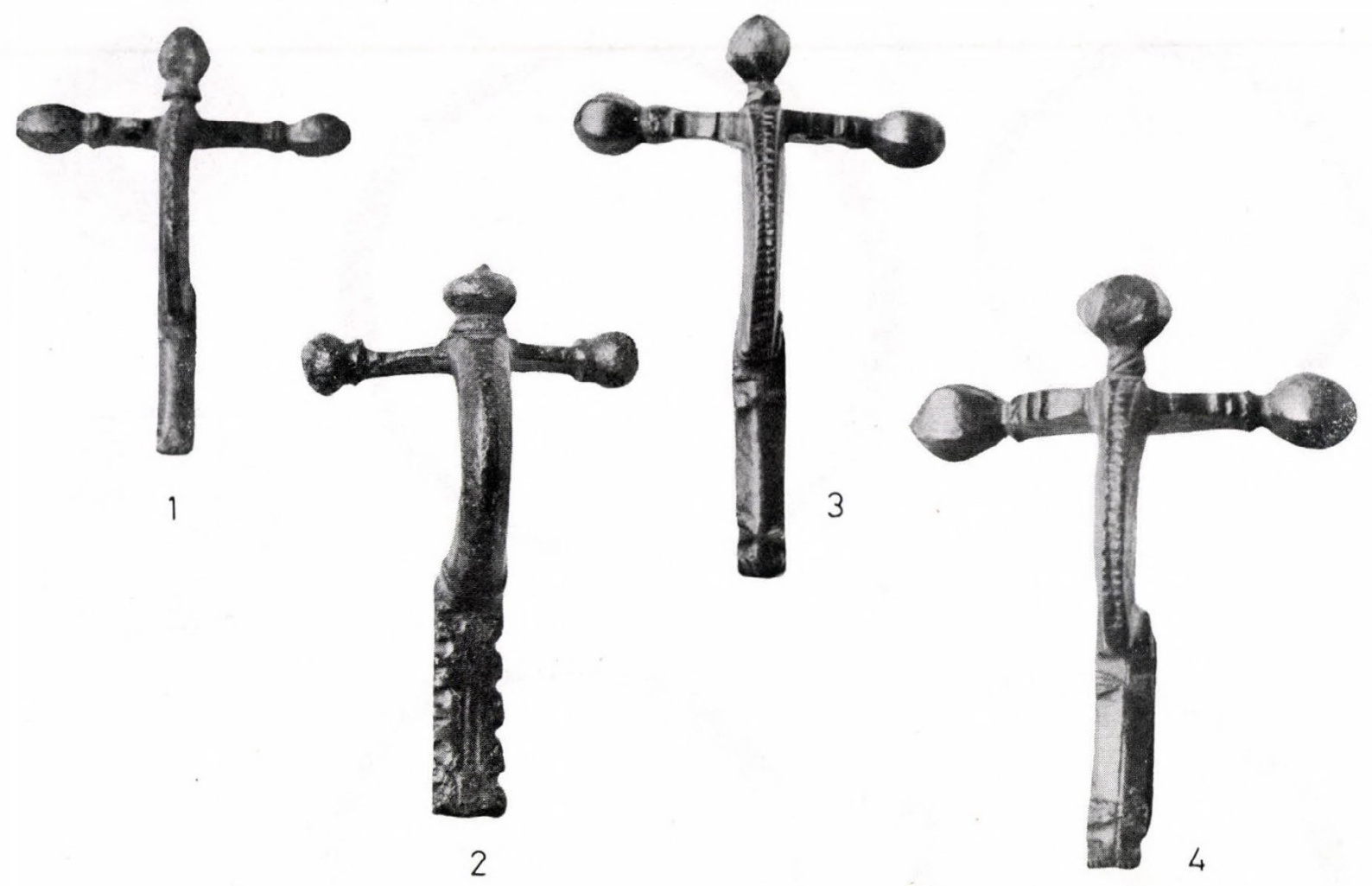

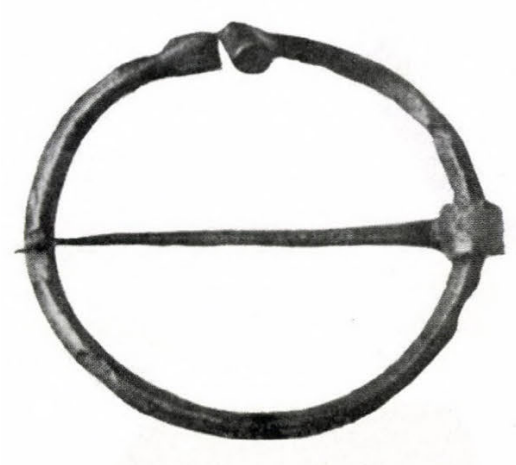

5

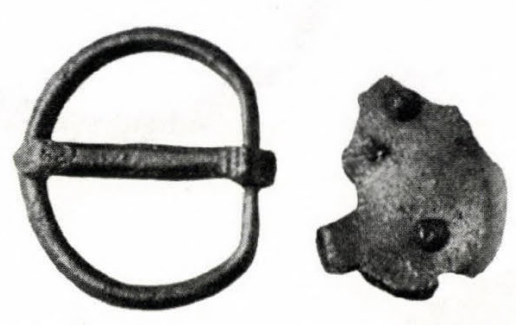

6

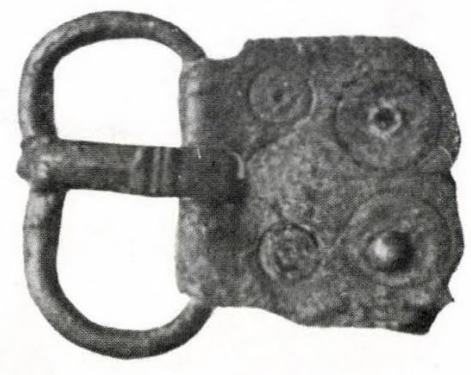

7
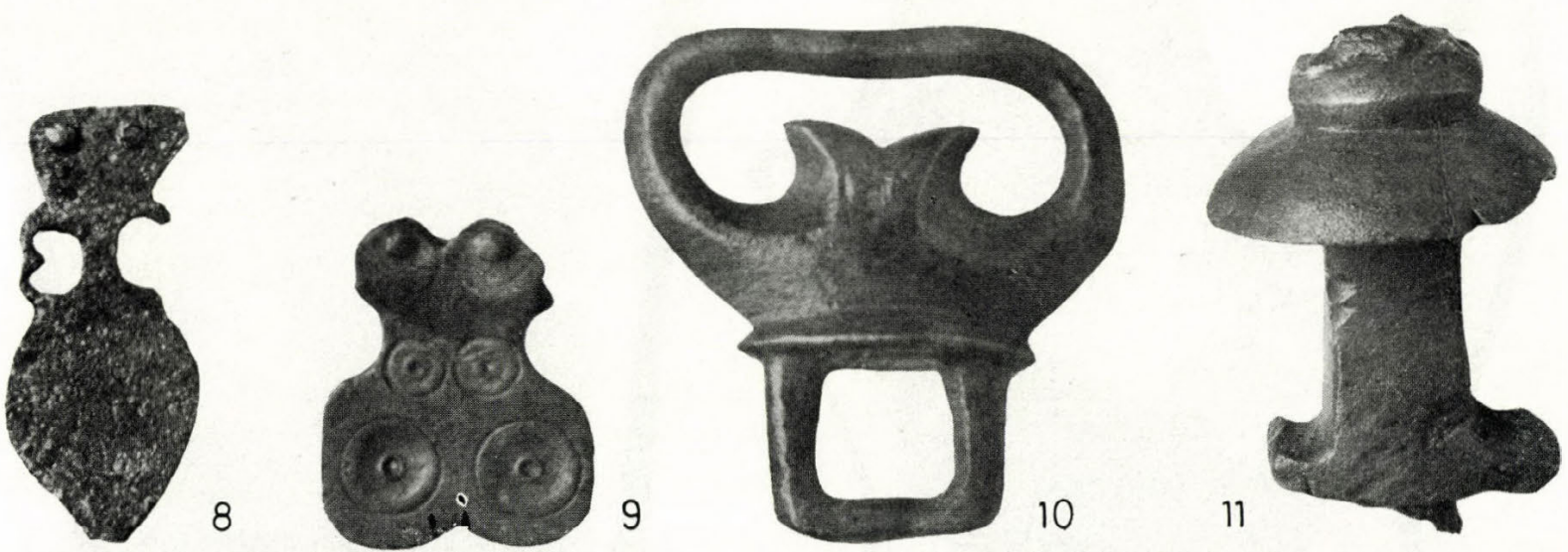

Plate 13 Graves R/151-163 (scale 1:1) 

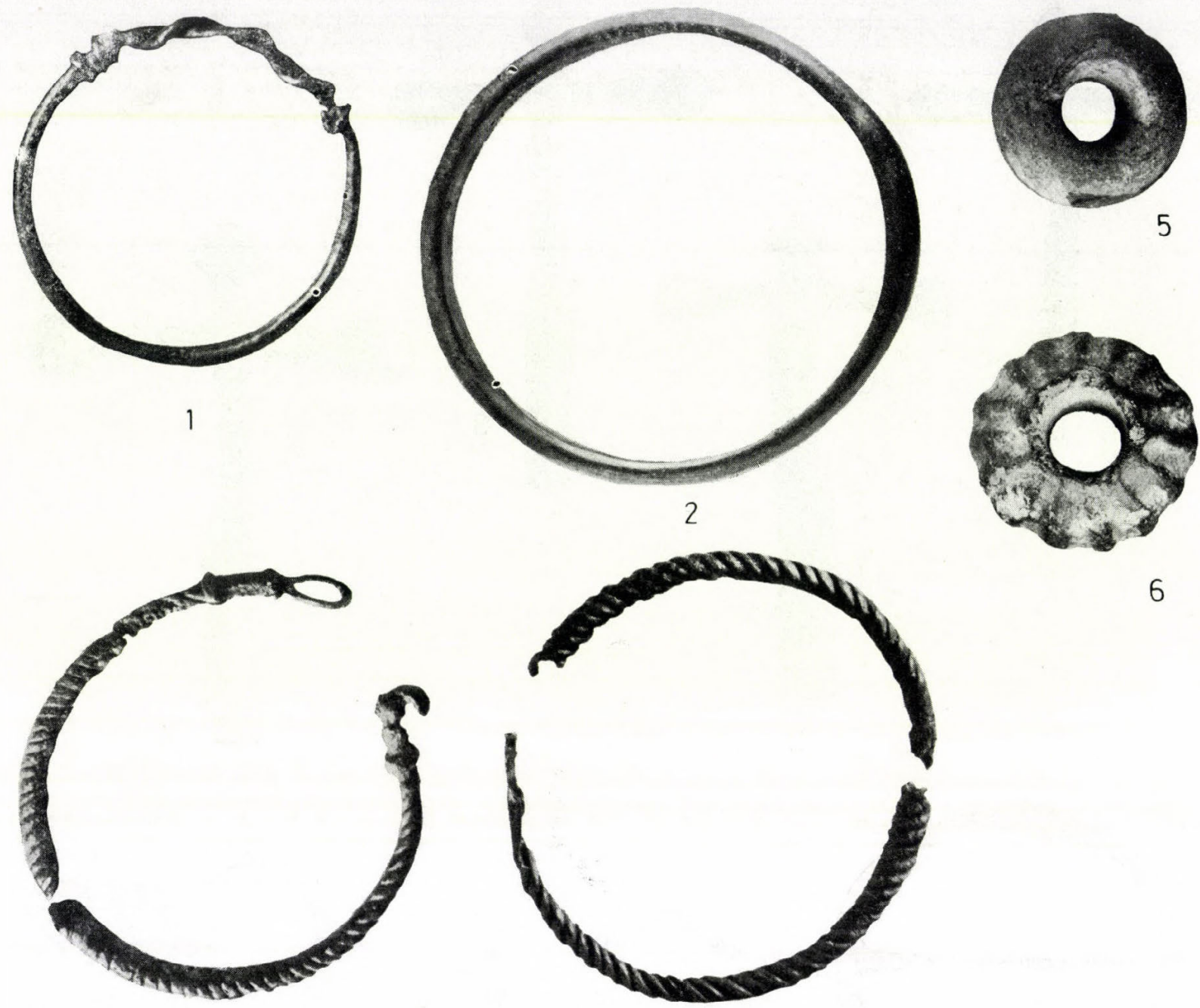

3
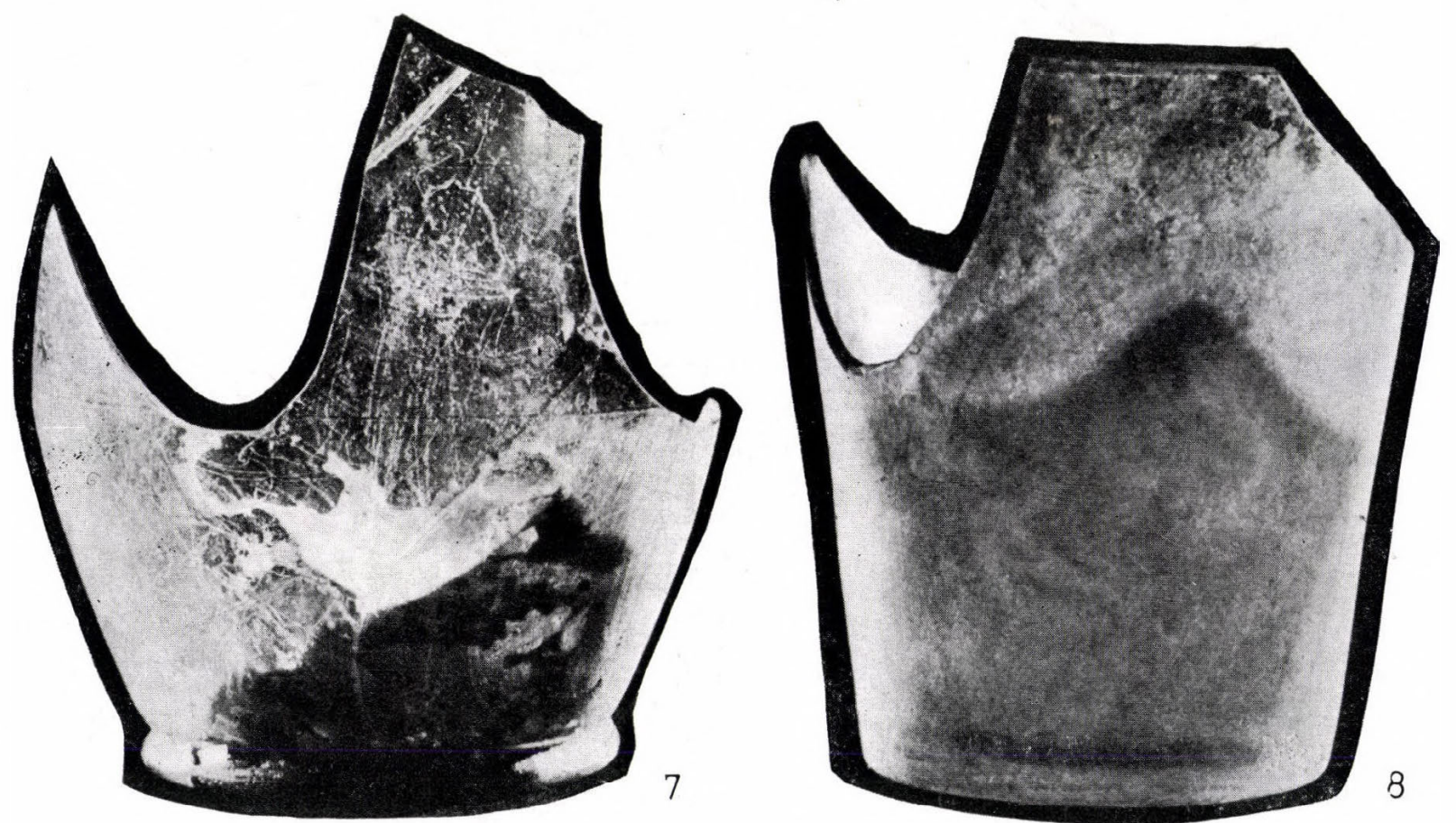

Plate 14 Graves R/151-163 (scale $1: 1$ ) 

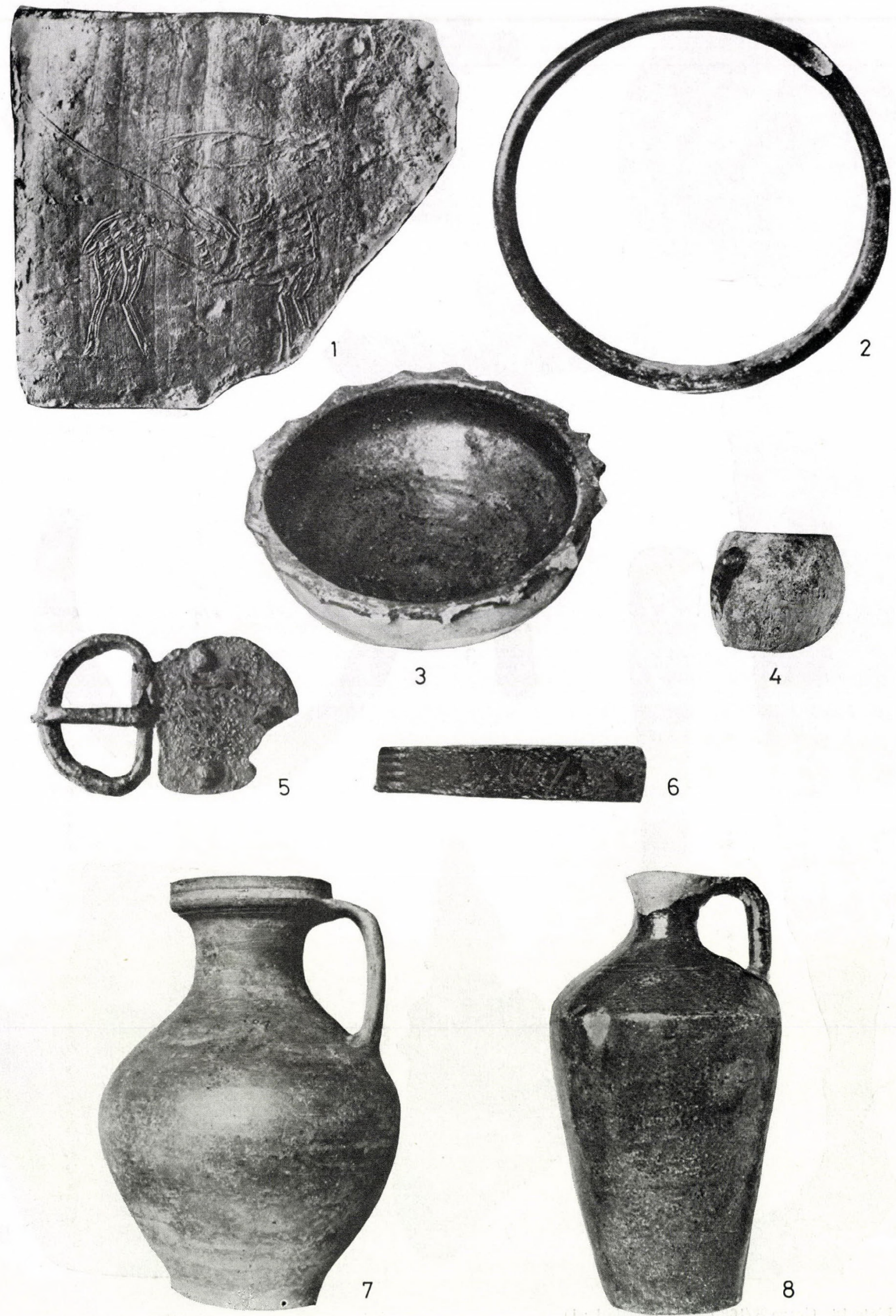

Plate 15 1: Graves R/125-145; 2: Grave R/146; 3, 4: Grave R/165; 5, 6: Grave R/166; 7: Grave R/169; 8: Grave R/167 


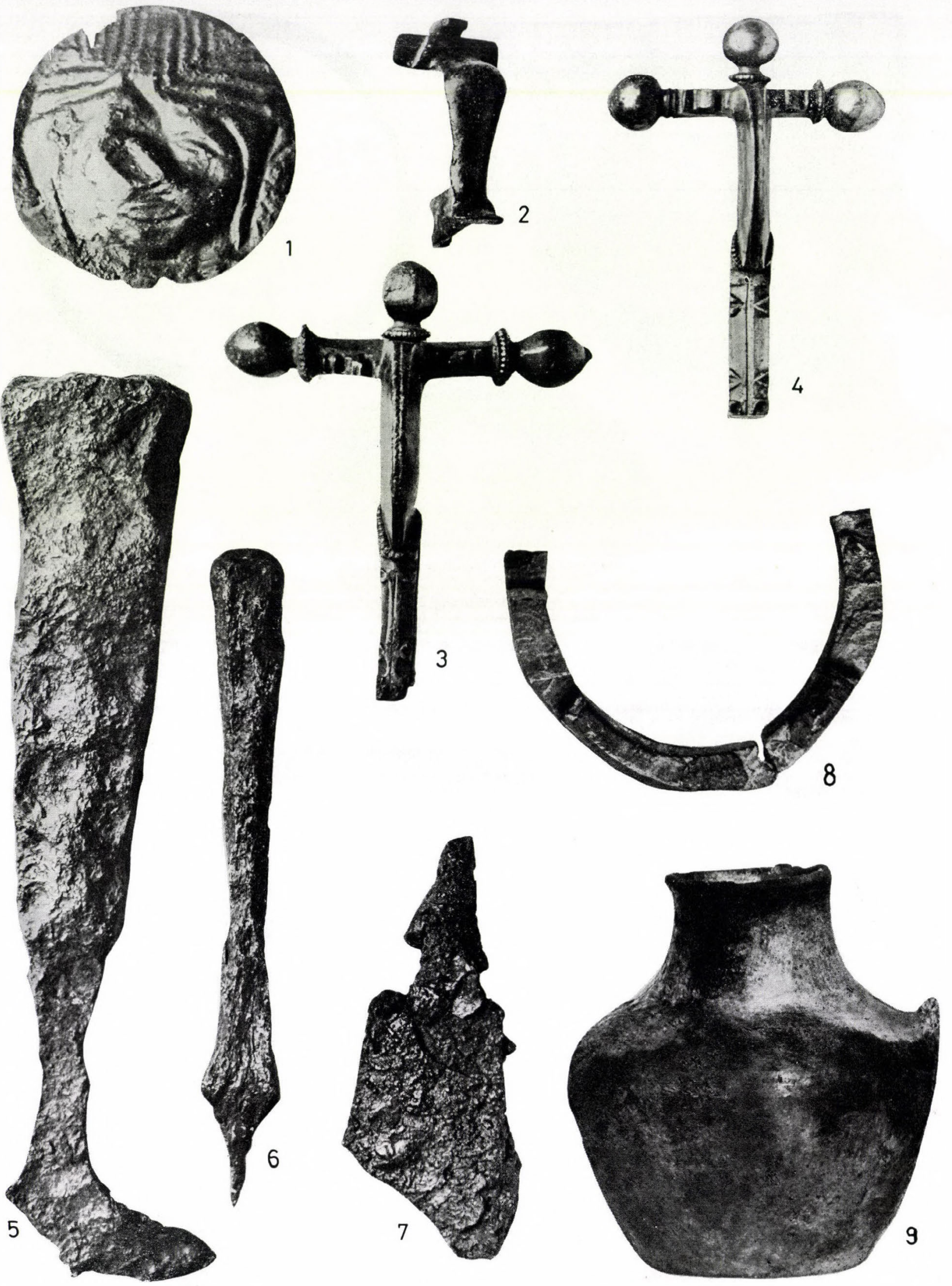

Plate 16 Grave R/168 (2: AR) (scale $1: 1$ ) 

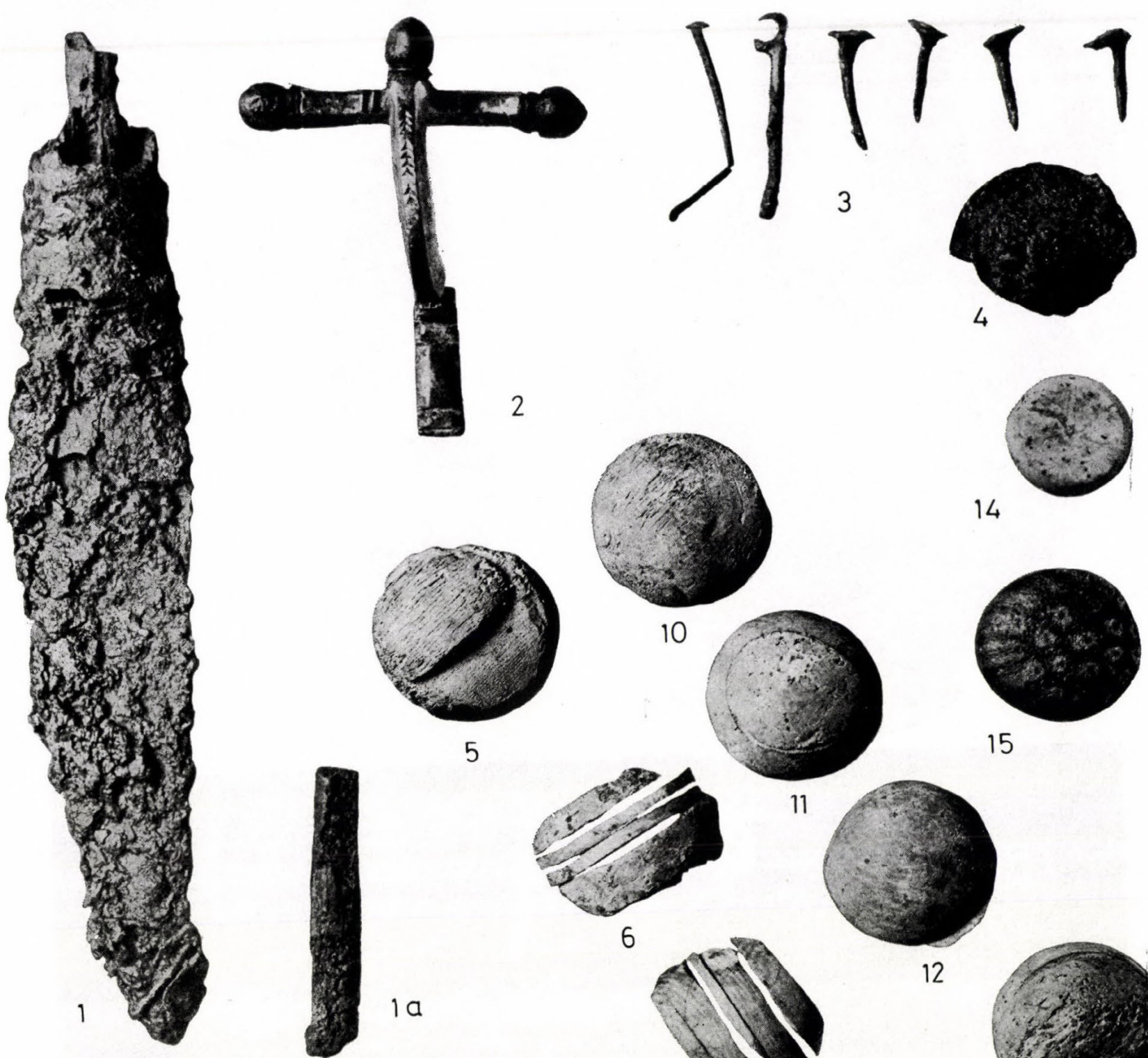

14

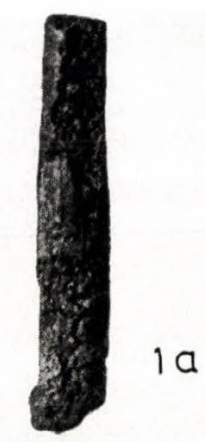

5
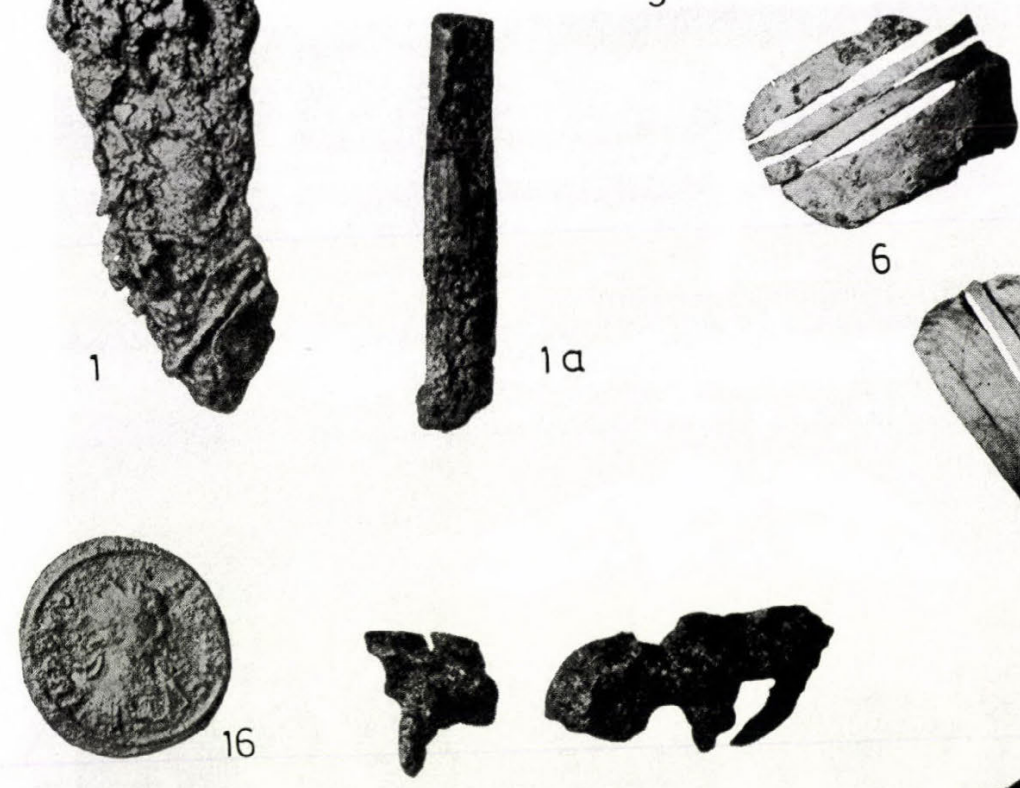

11
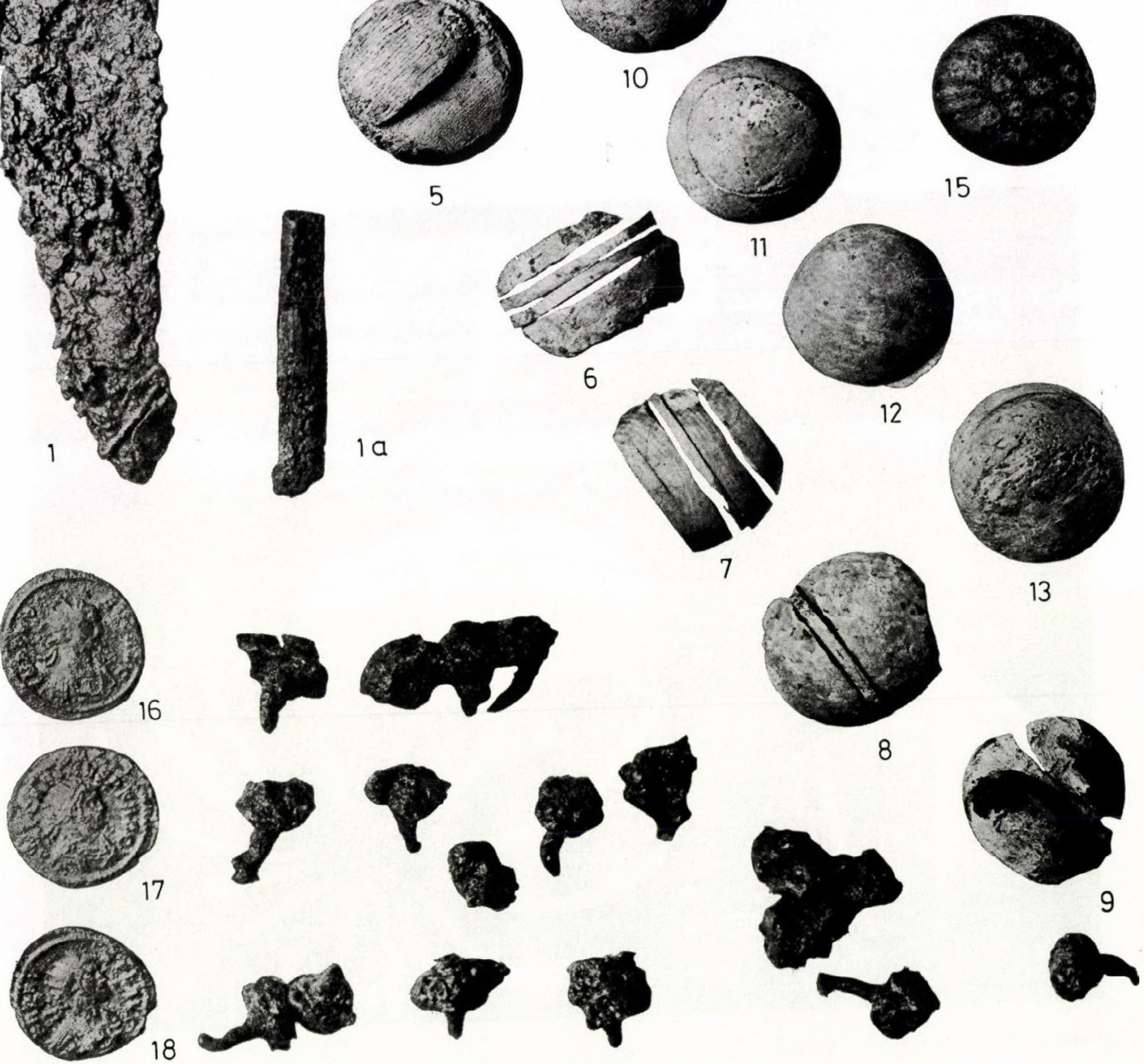

Plate 17 Grave $R / 170$ (scale $1: 1$ ) 

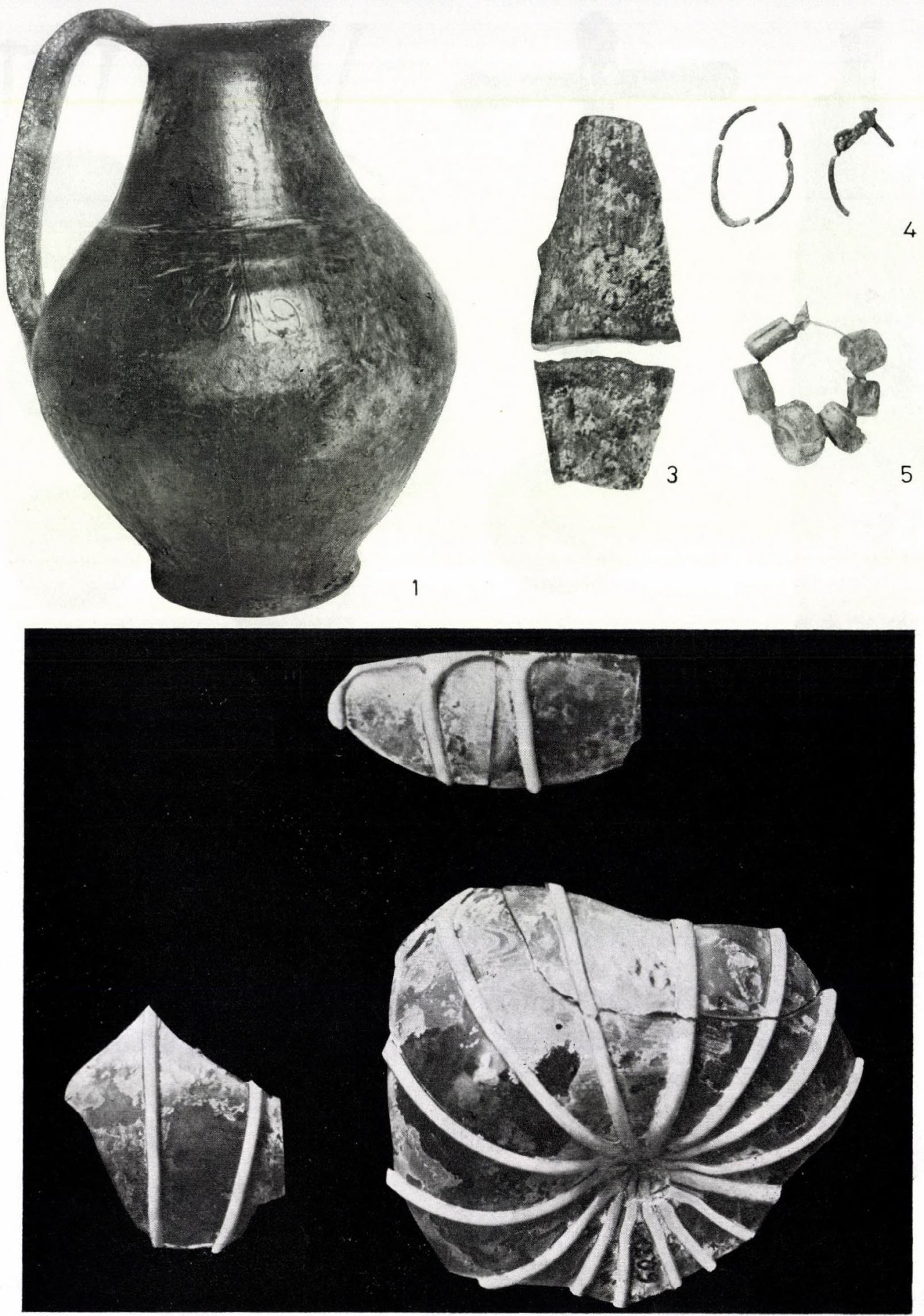

Plate 18 1, 2: Grave R/177; 3, 4: Grave R/185; 5: Grave R/187 

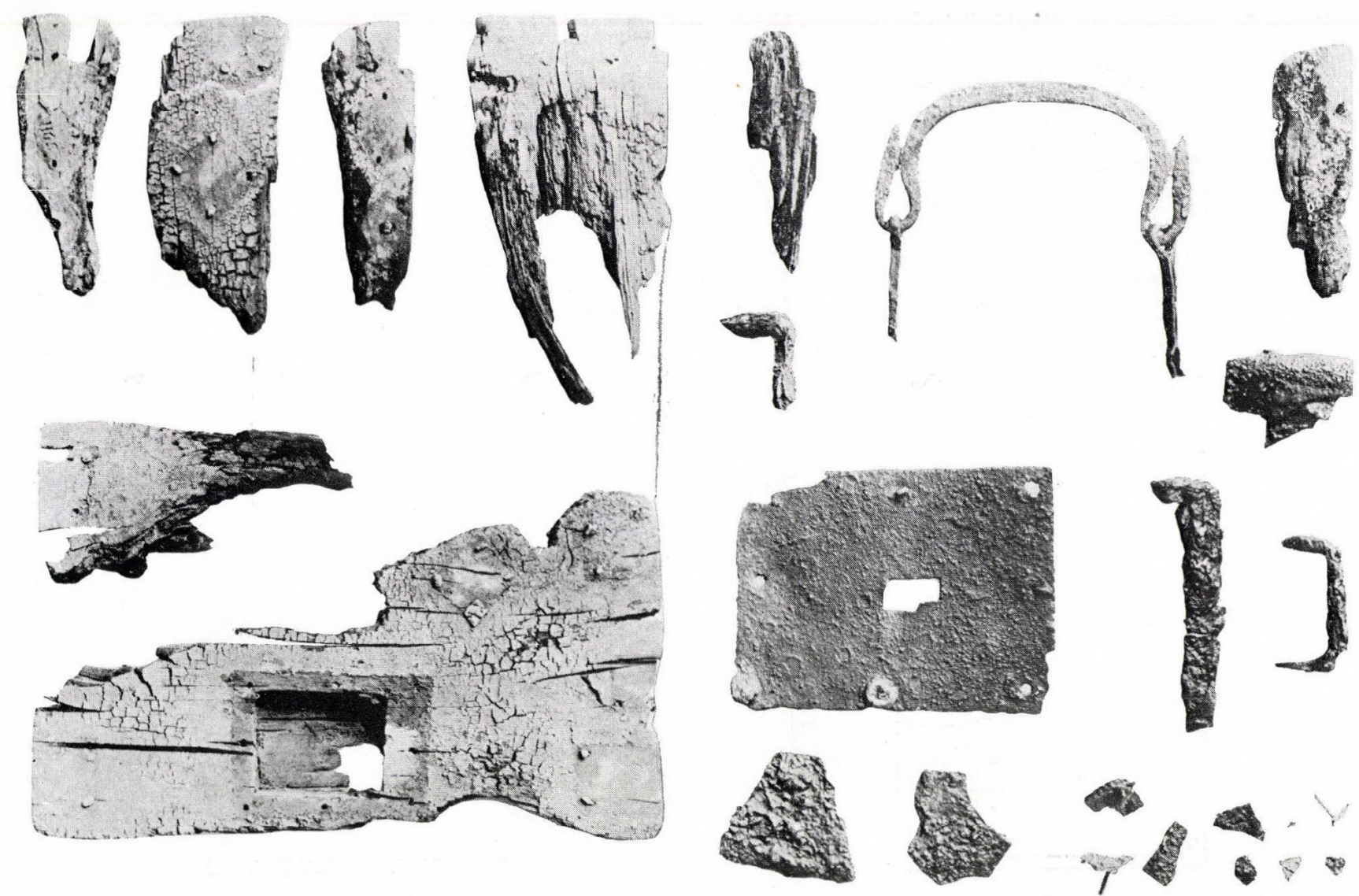

1

\section{2}
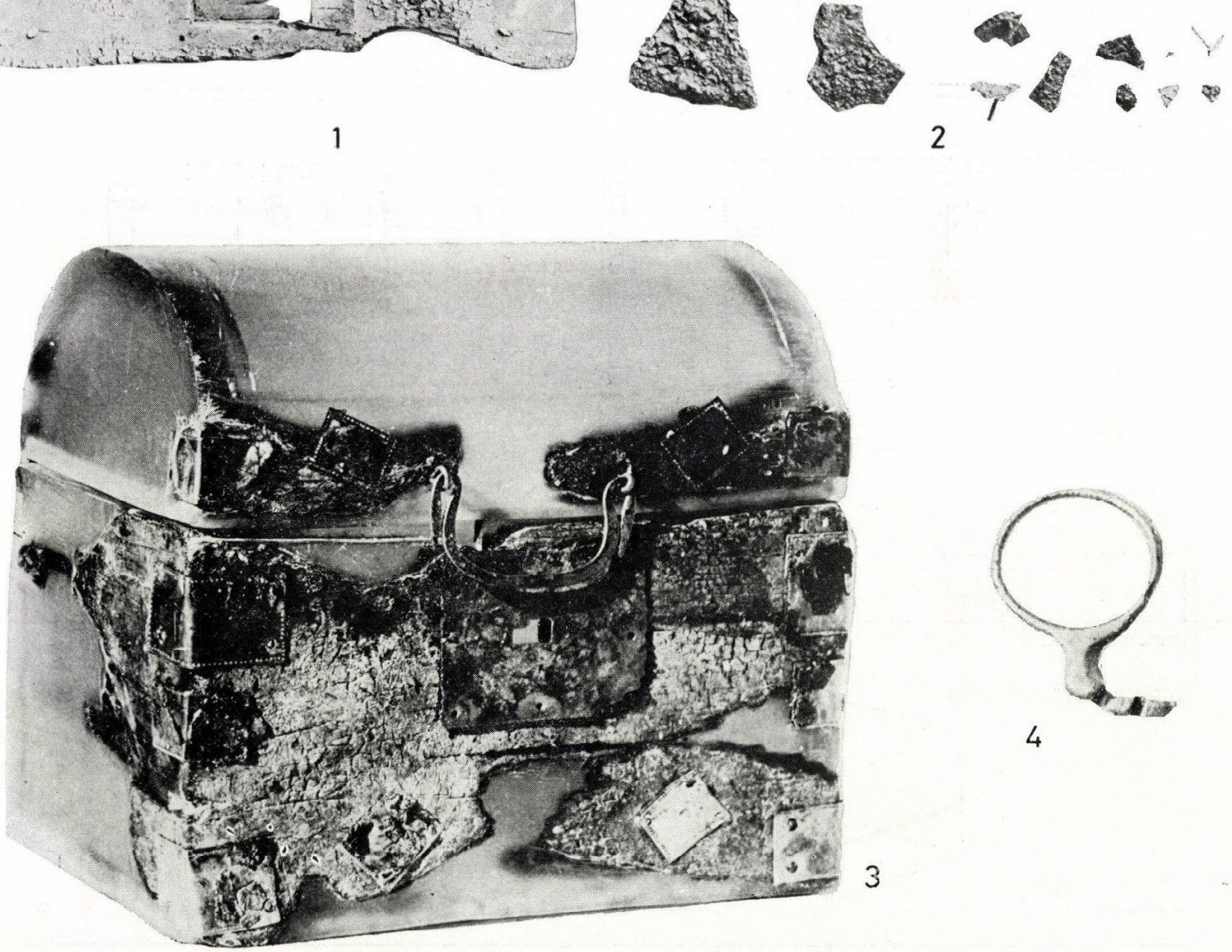

Plate 19 Grave R/177. 1-2: Remains of wooden casket-before restoration; 3: Same after restoration - reconstruction; 4: Ringed key from the grave 

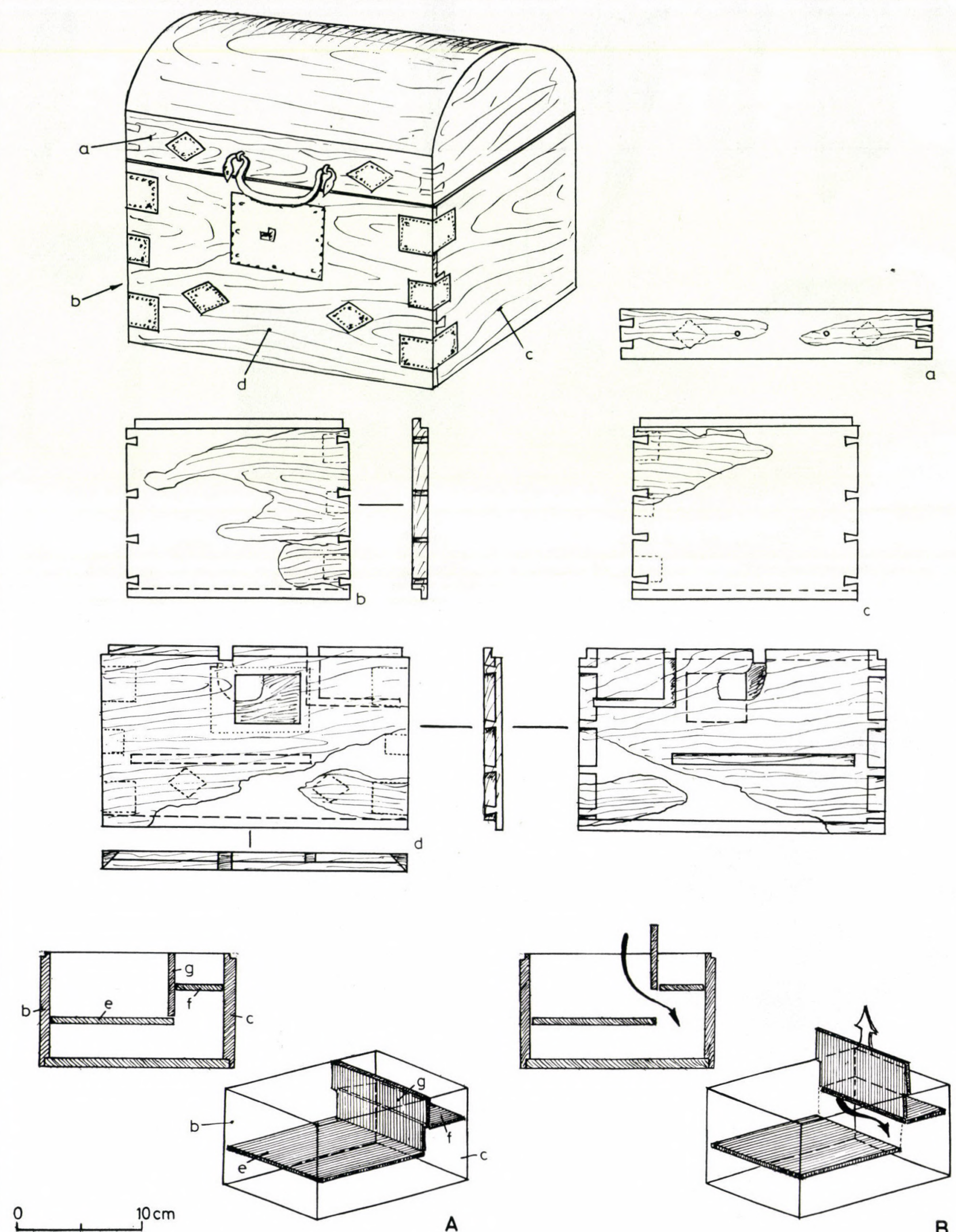

0

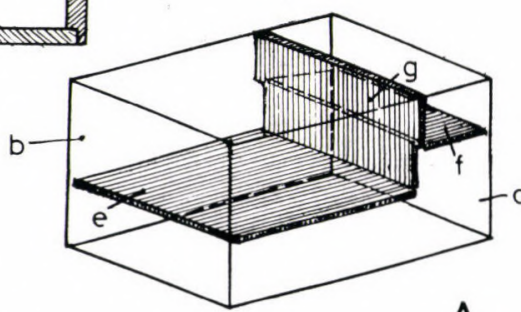

A

Plate 20 Grave R/177: Reconstruction of wooden casket 

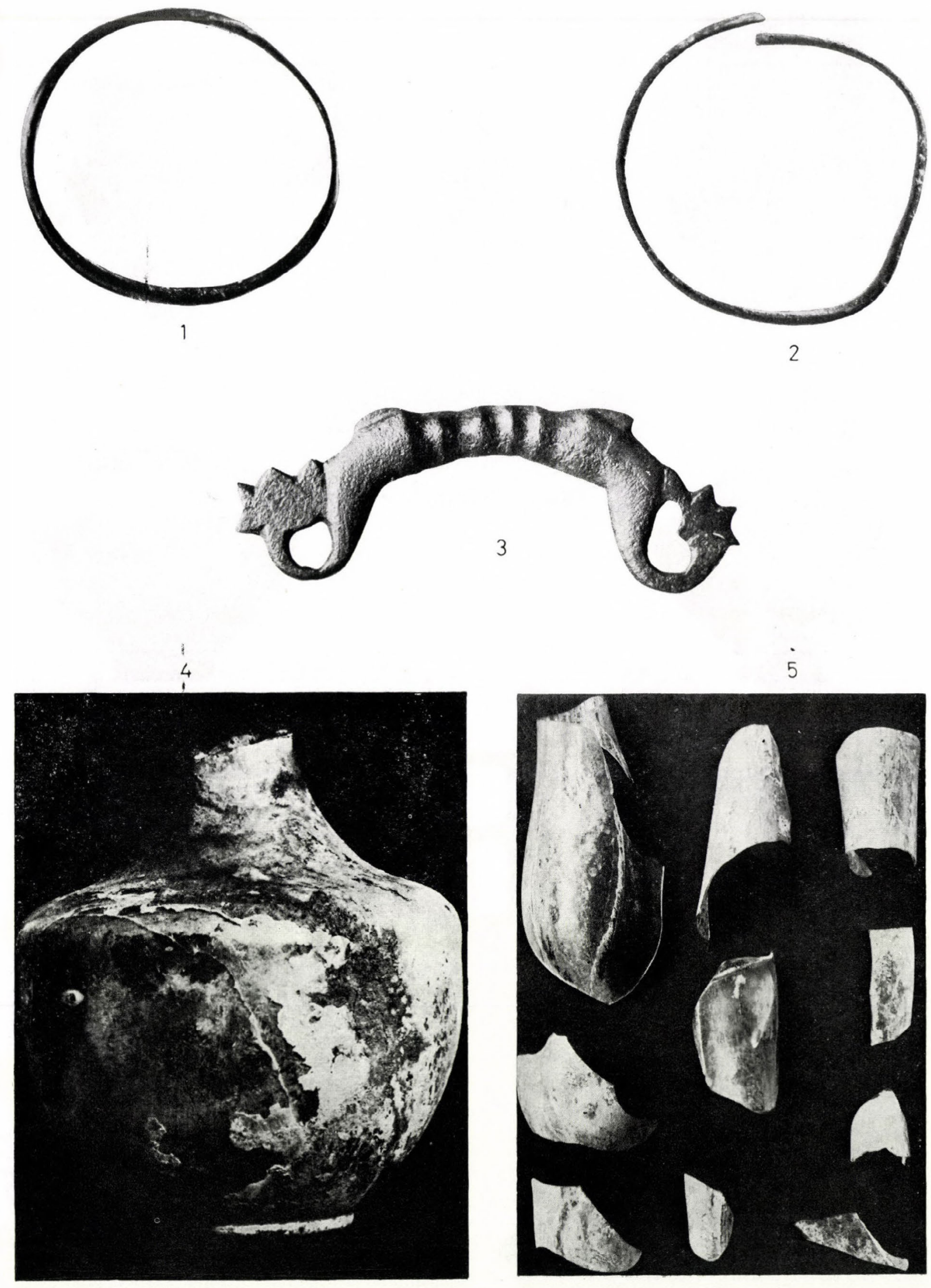

Plate 21 Grave R/181 

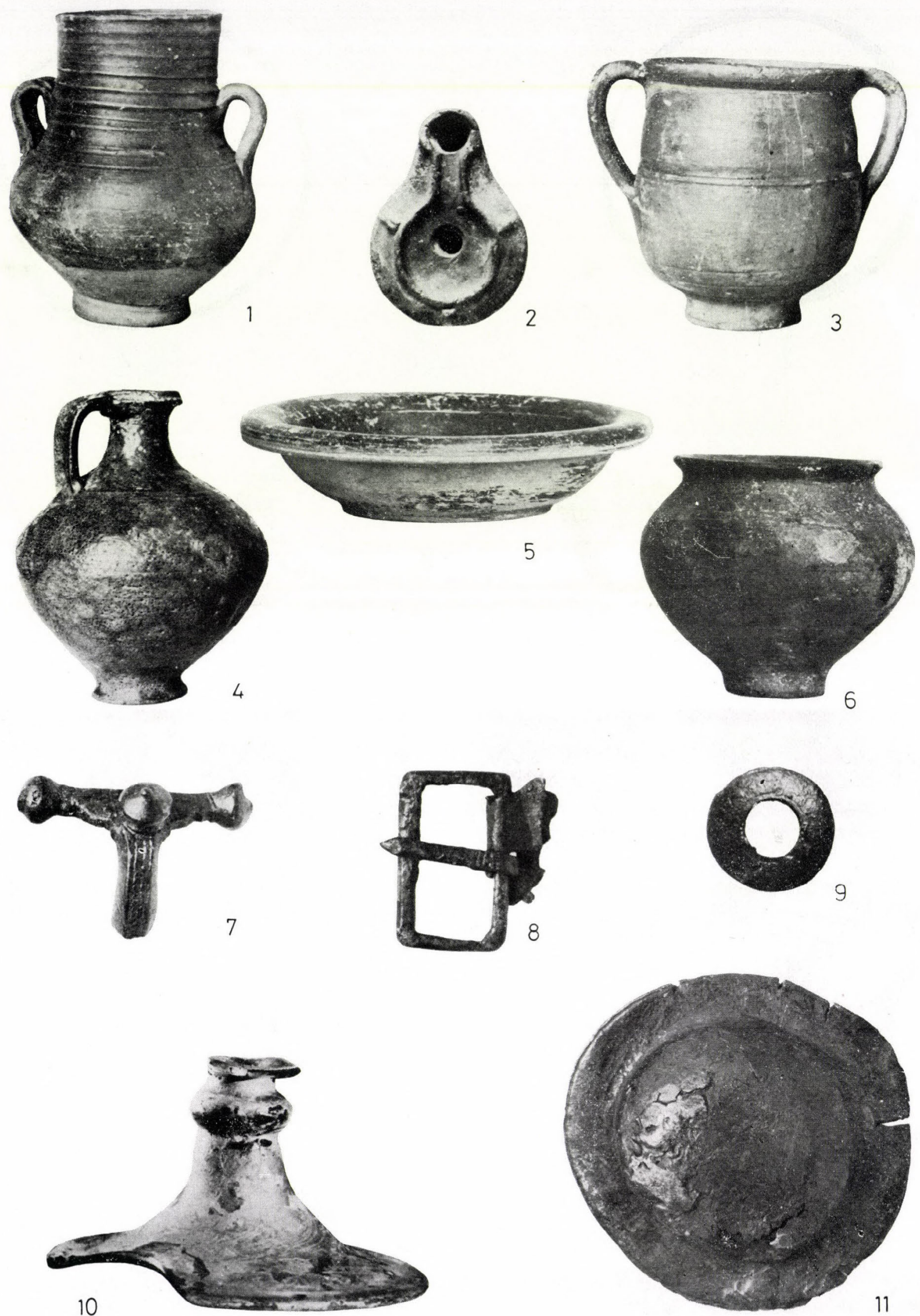

Plate 22 1, 3, 4, 5: Grave R/181; 2, 6-11: Grave R/182 

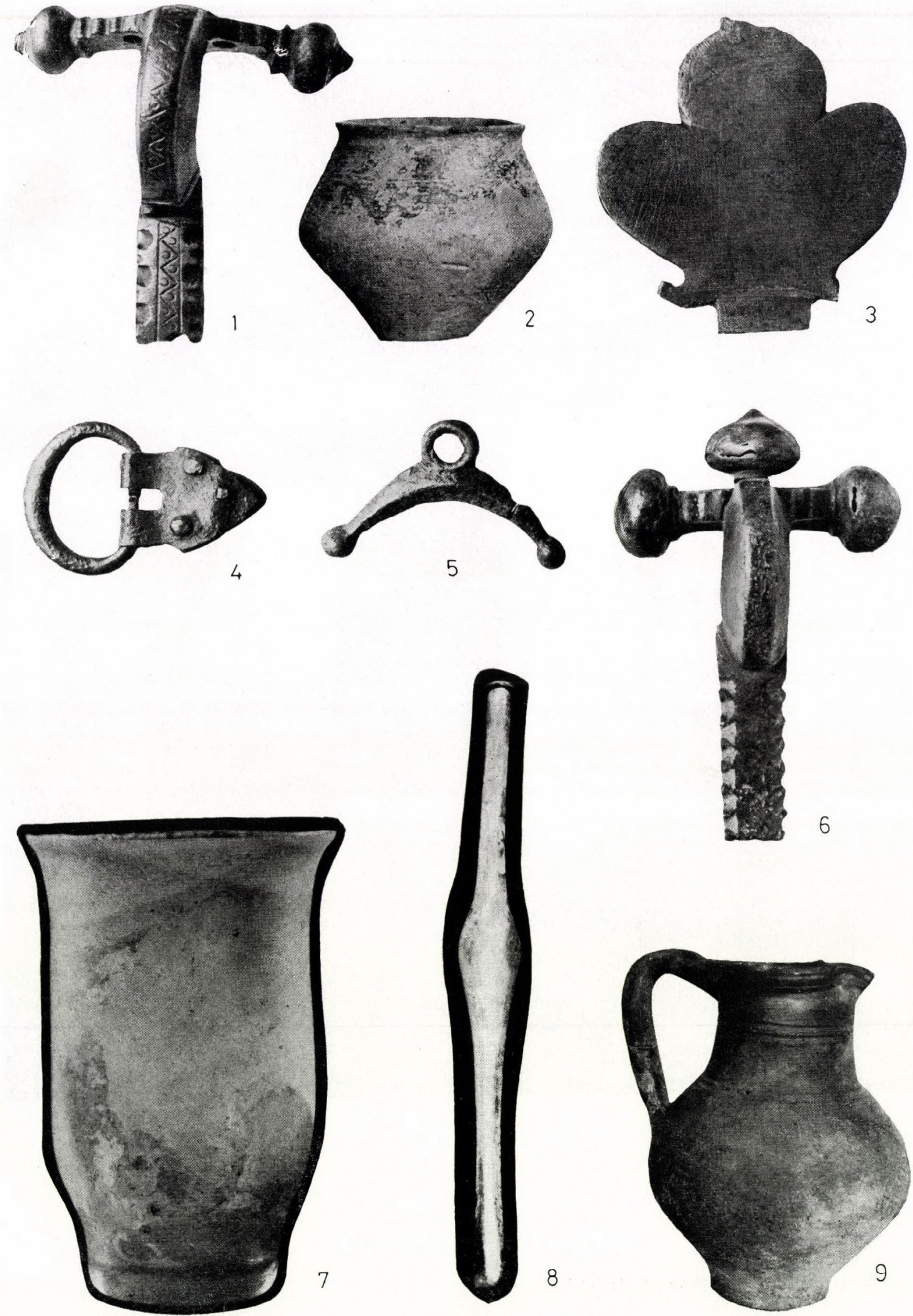

Plate 23 1-3: Grave R/183; 4, 7, 8: Grave R/184; 5, 6, 9: Grave R/190 


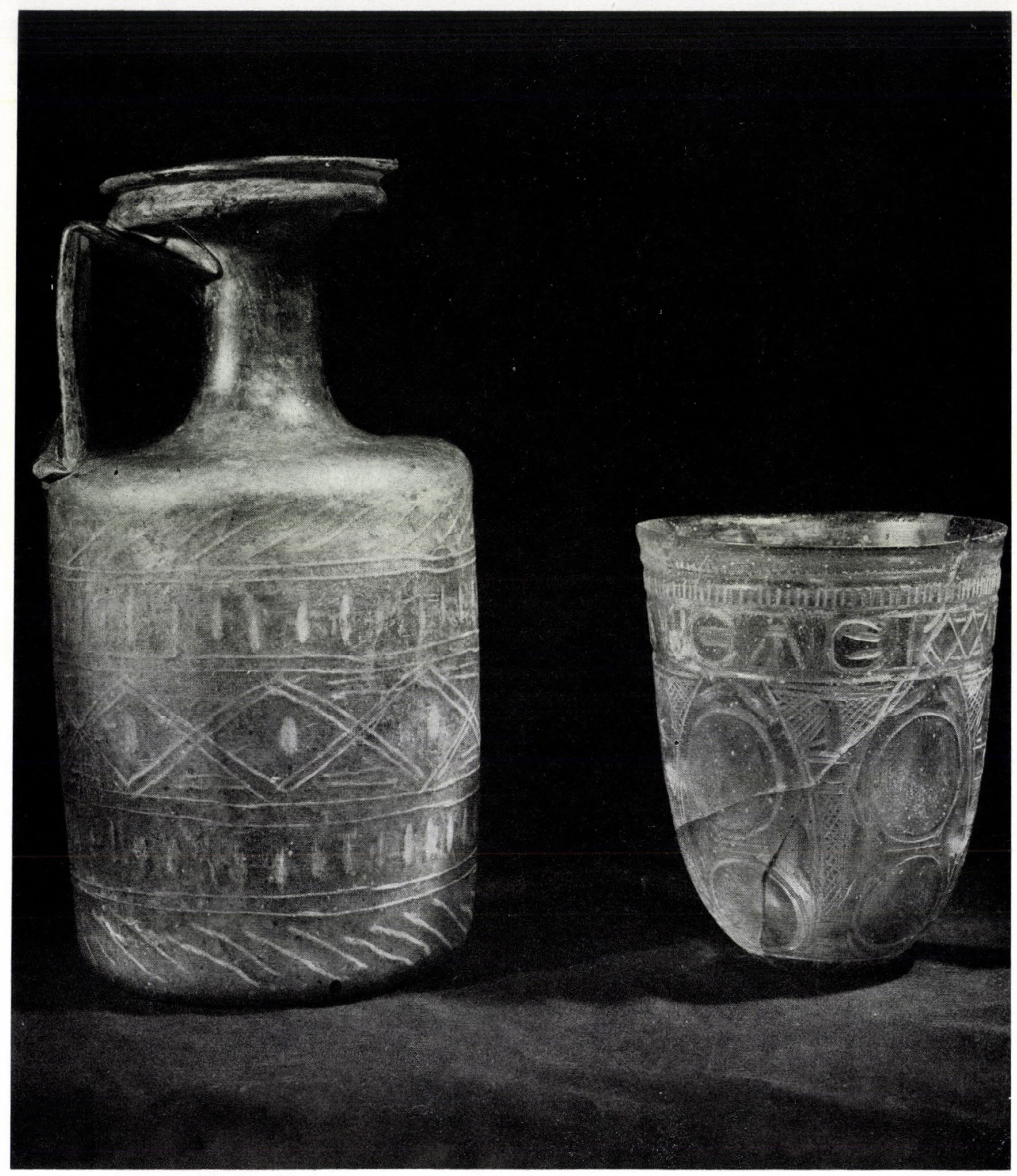

Plate 24 1: Glass jug from Grave R/191; 2: Glass beaker from Grave R/192 

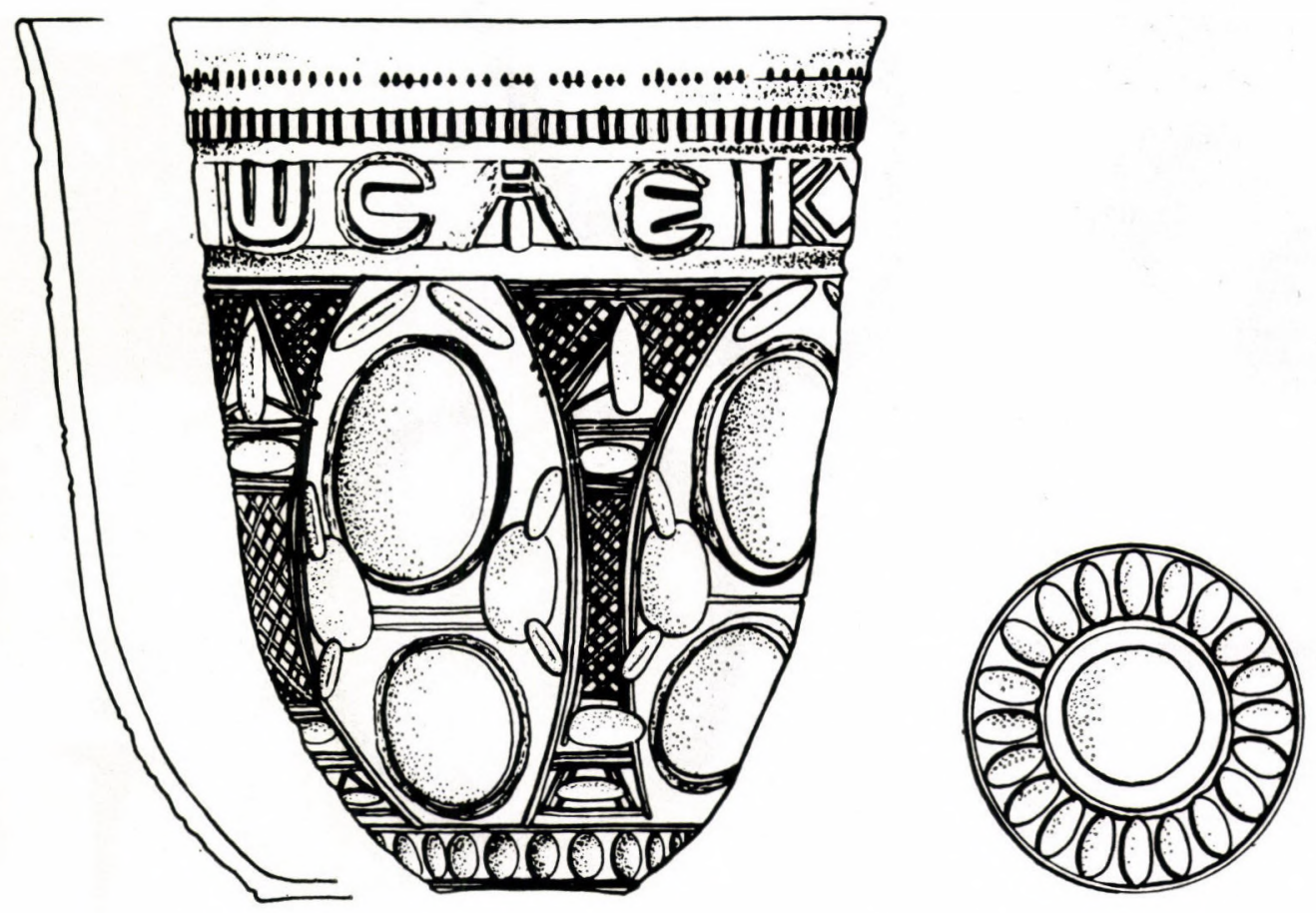

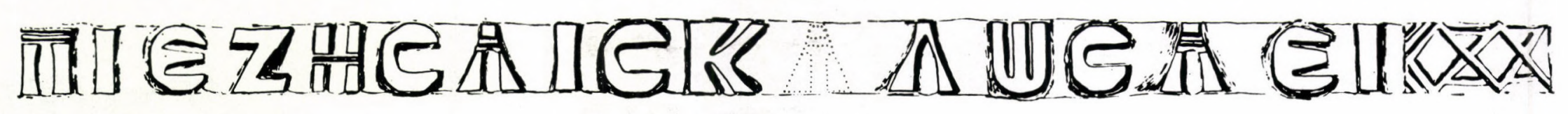

Plate 25 Glass beaker with engraved decoration and Greek inscription from Grave R/192 

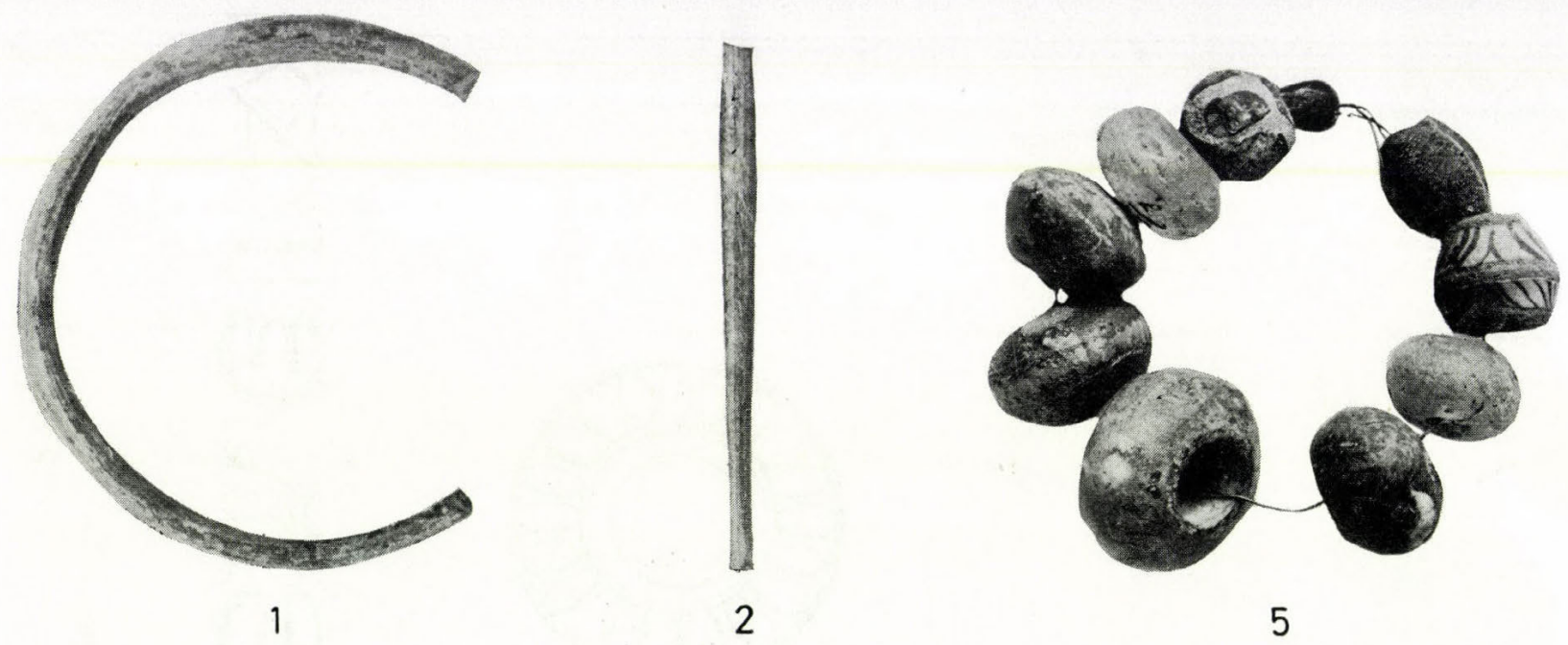

2

5
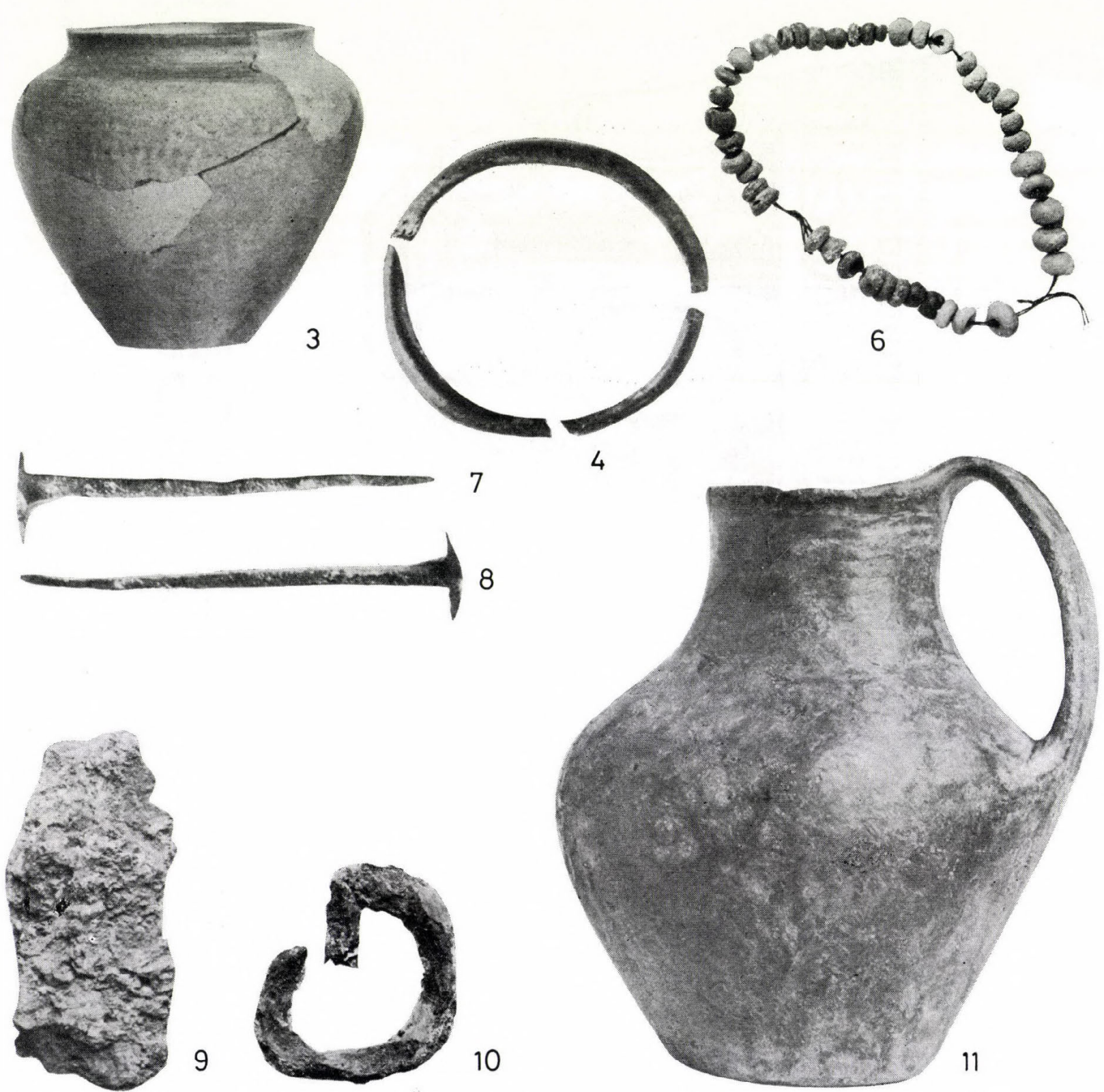

Plate 26 1-2: Grave R/169; 3: Grave R/222; 4: Grave R/227; 5-6: Grave R/193; 7-8: Grave R/223; 9-11: Grave R/272 

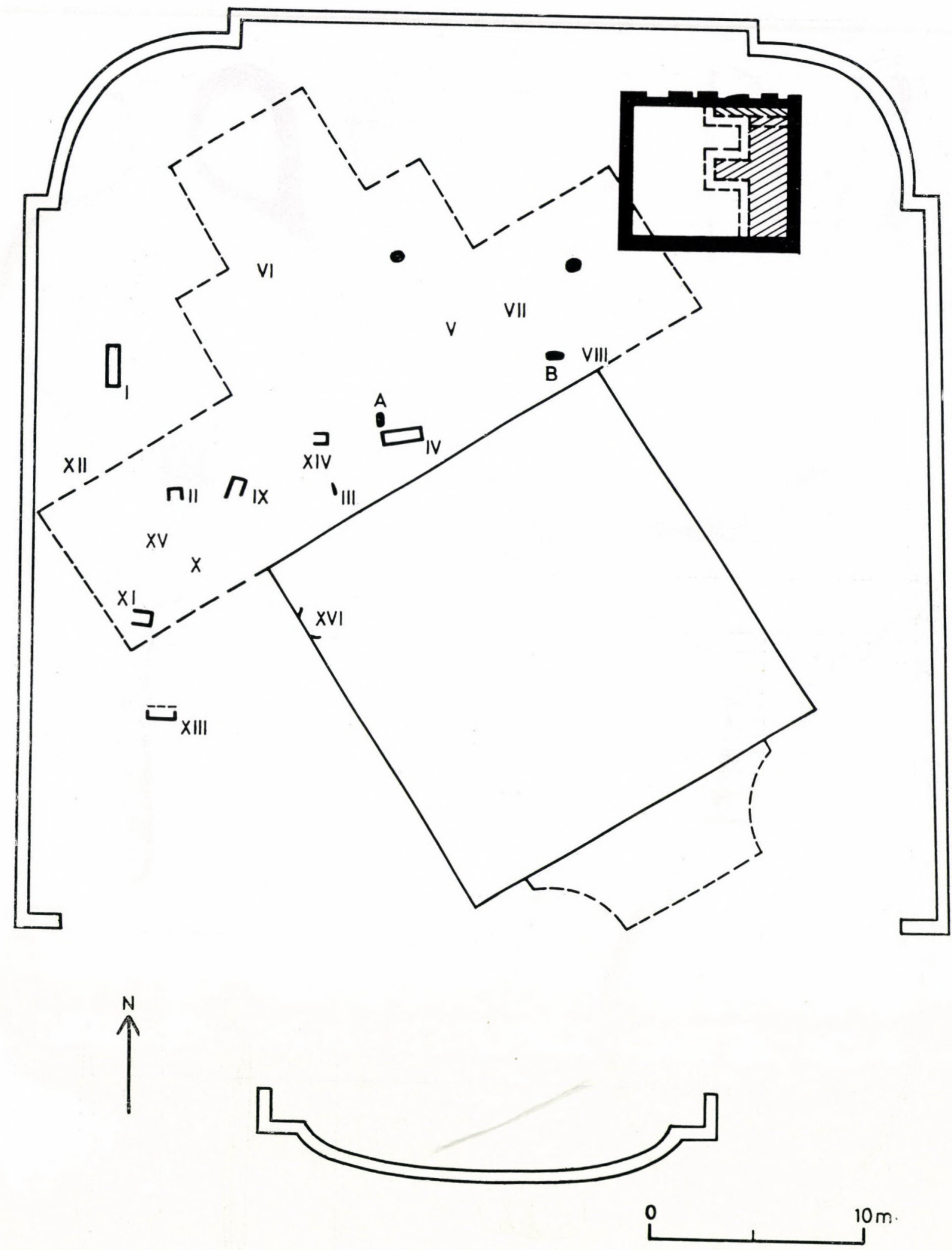
Plate 27 Széchenyi Square-City Parish Church (1939). Ground plan of the excavation of the cemetery.
(Graves R/202-217 correspond to Gy. Török's Graves I-XVI) 


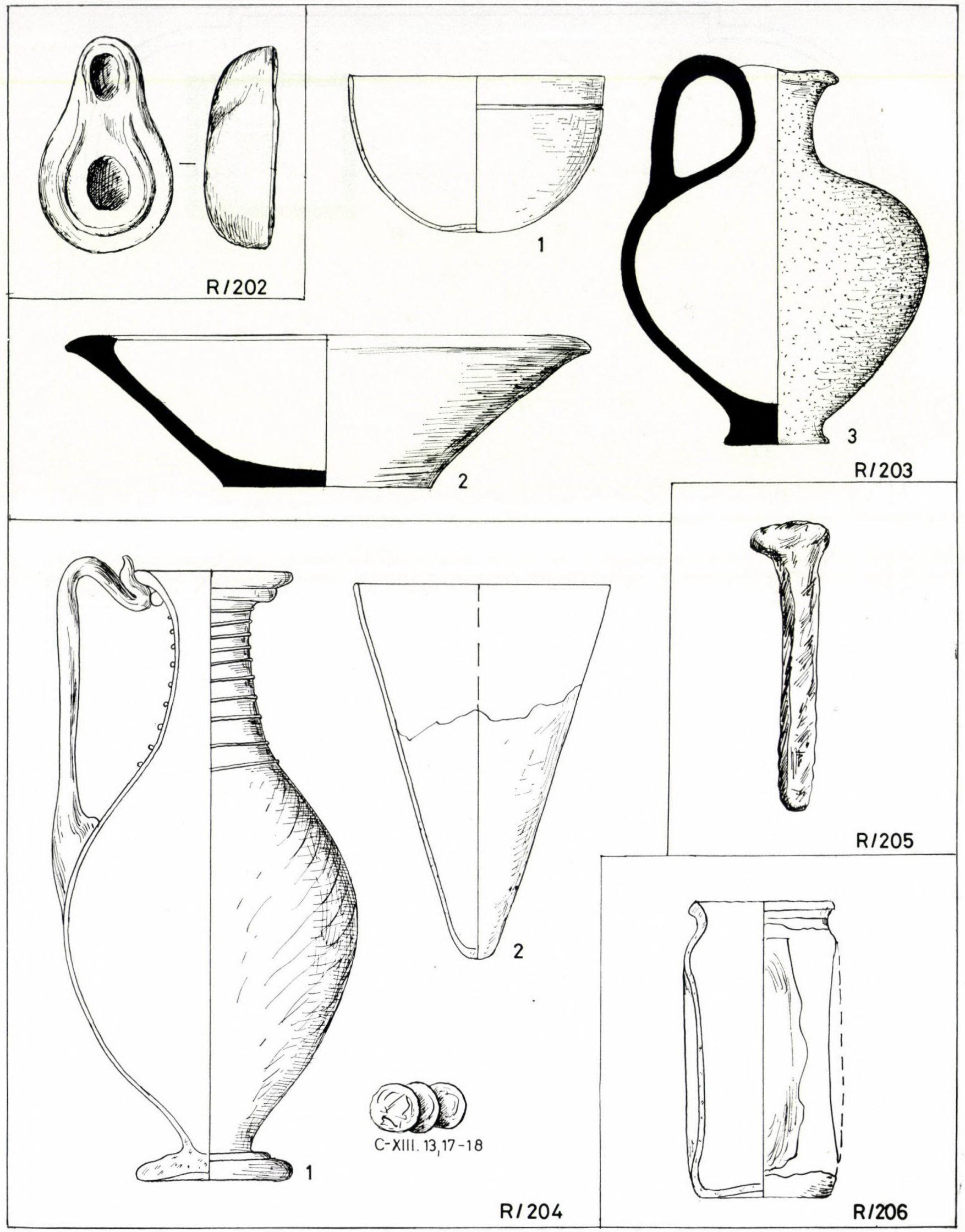

Pla te 28 Széchenyi Square-City Parish Church (1939). Graves R/202-206 (scale 1 : 2) 


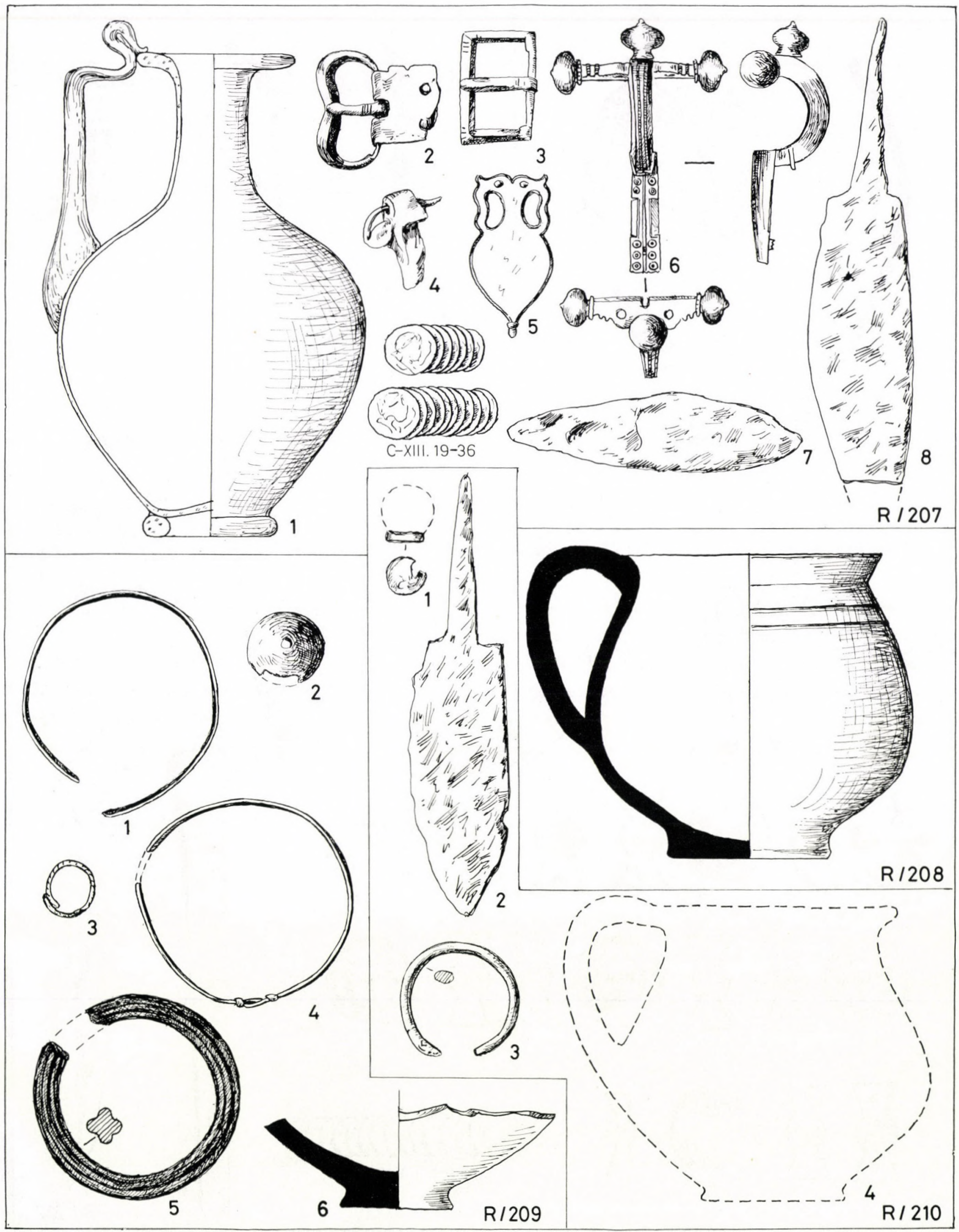

Pla te 29 Széchenyi Square-City Parish Church (1939). Graves R/207-210 (scale $1: 2$ ) 


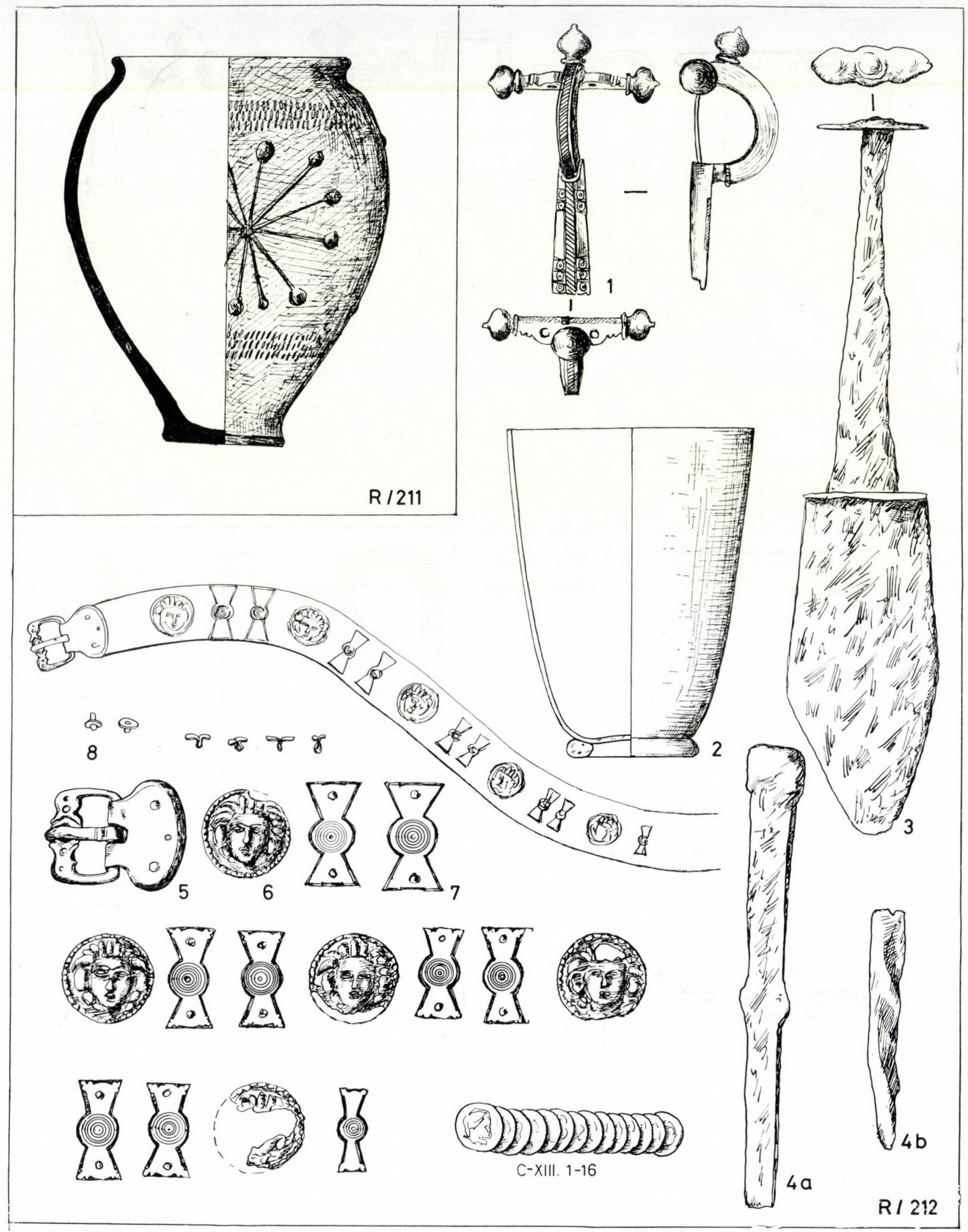

Plate 30 Széchenyi Square-City Parish Church (1939). Graves R/211-212 (scale 1 : 2) 


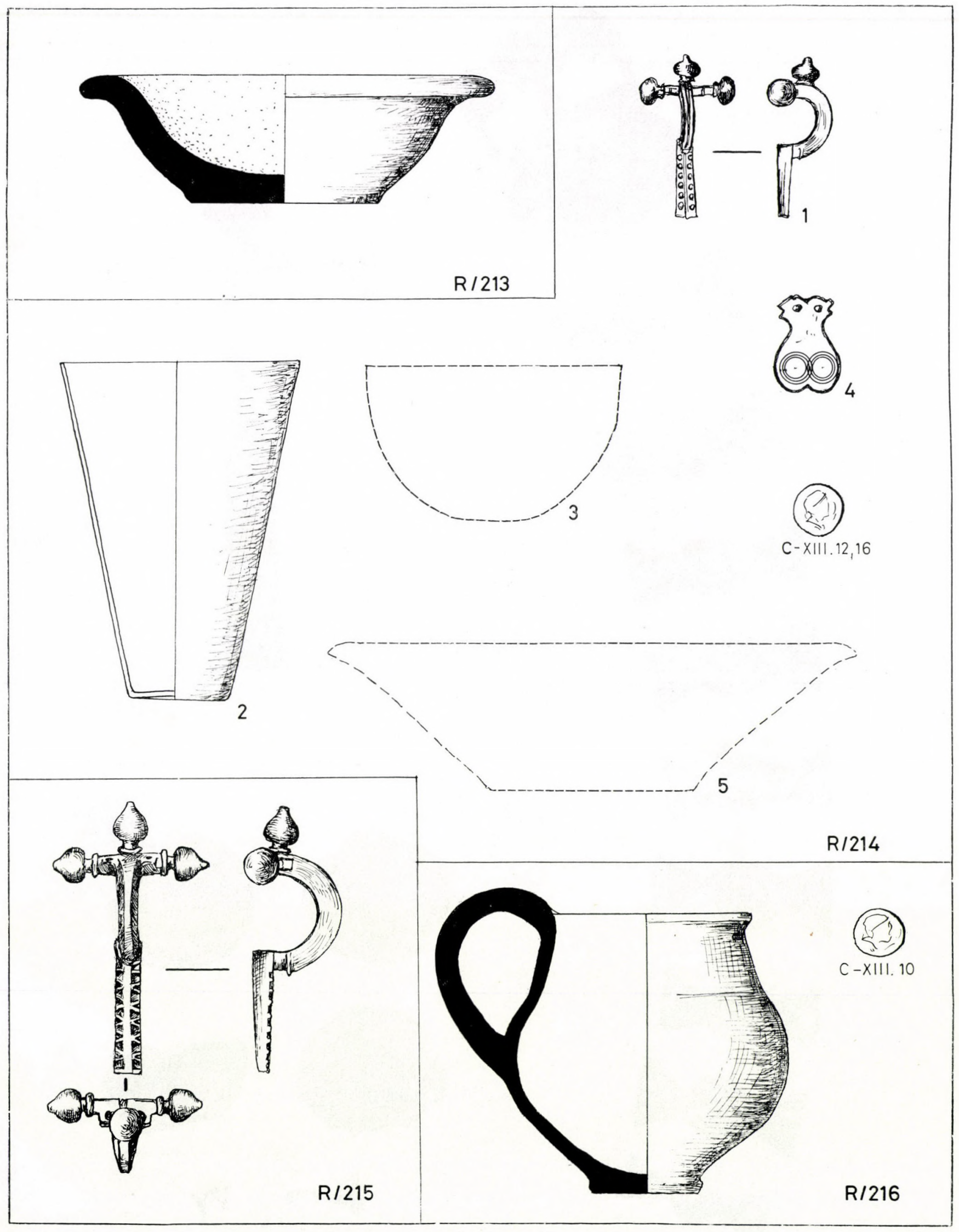

Plate 31 Széchenyi Square-City Parish Church (1939). Graves R/213-216 (scale 1:2) 

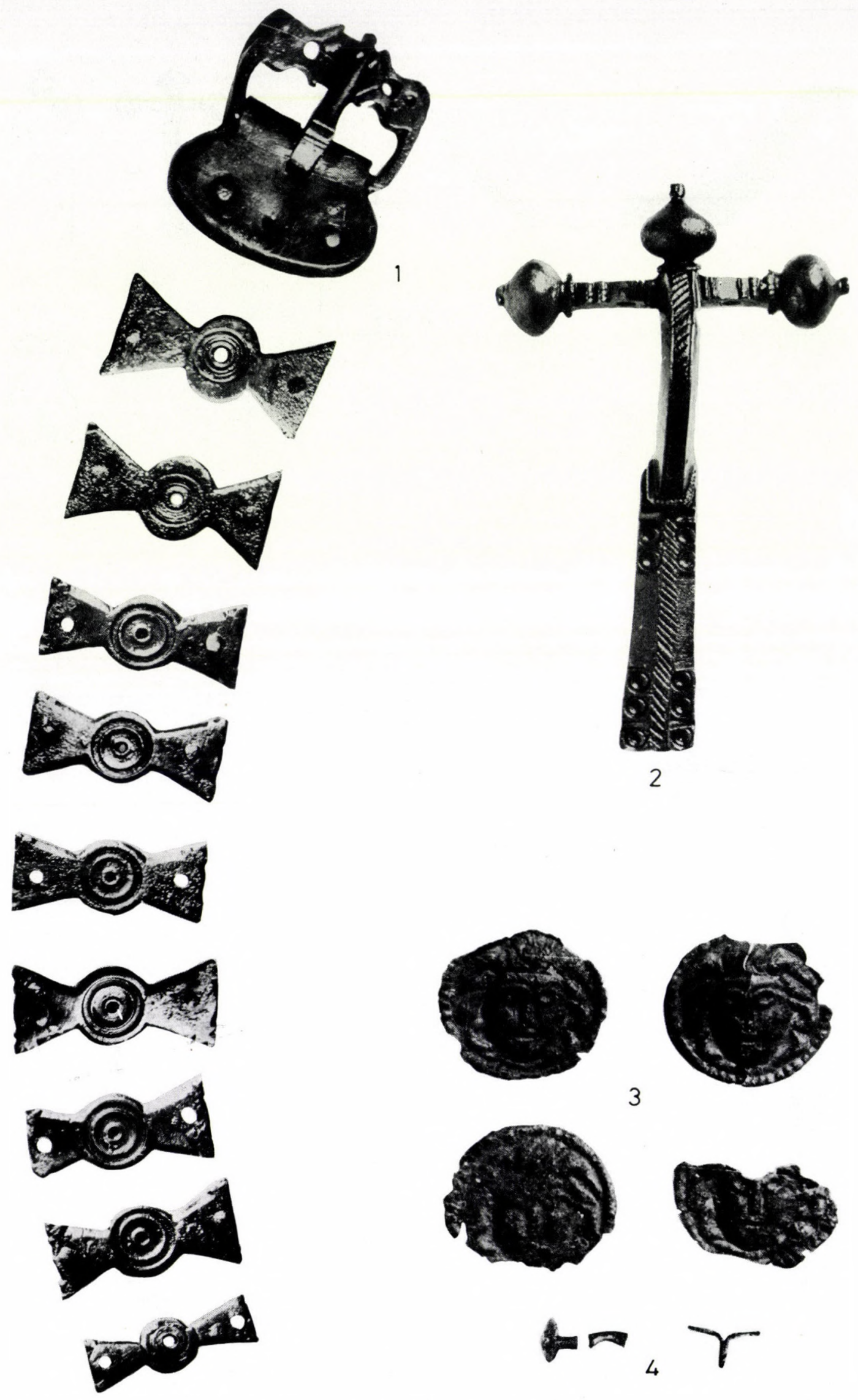

Plate 32 Széchenyi Square-City Parish Church (1939). Grave R/212 (scale $1: 1$ ) 

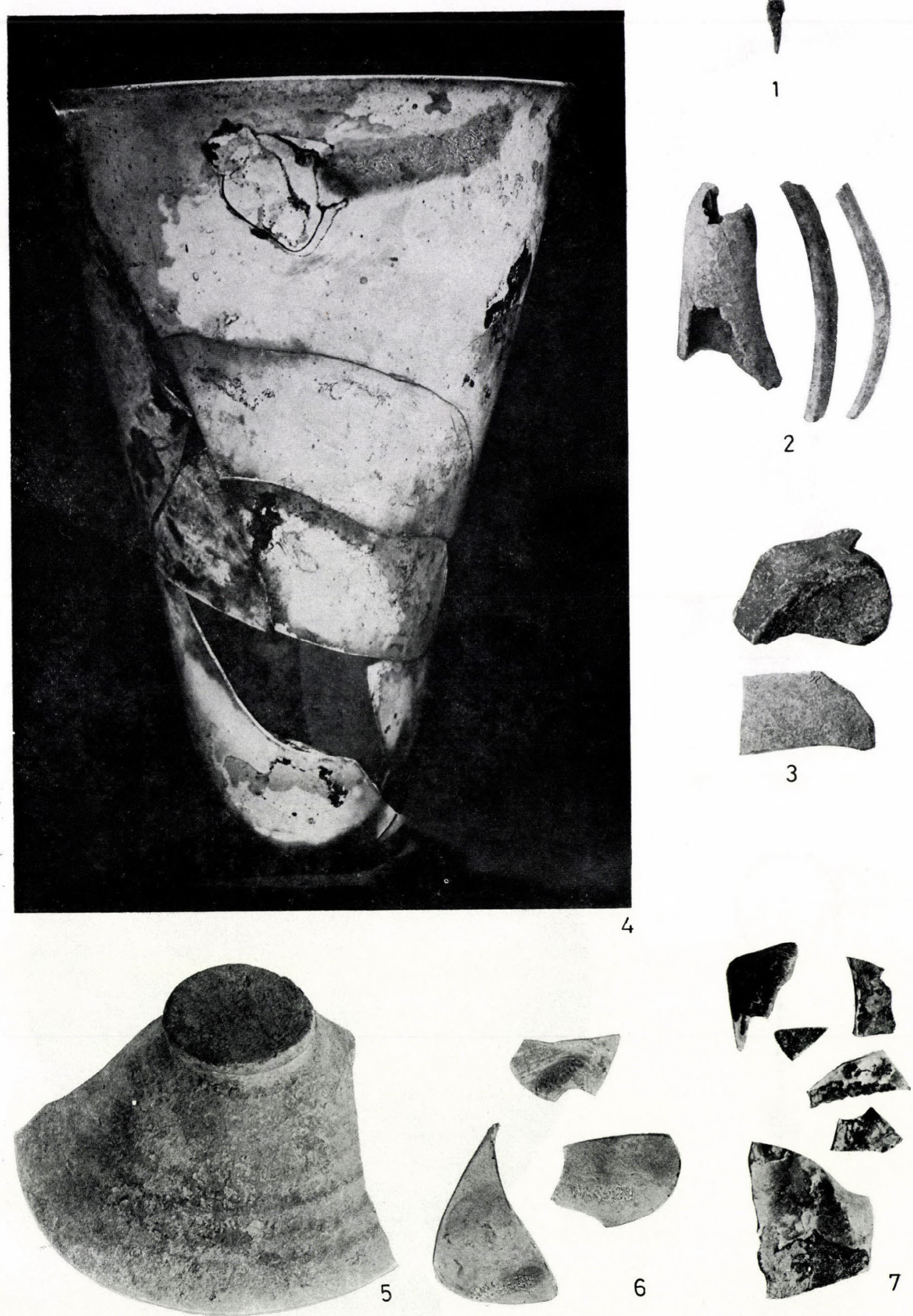

Plate 33 Rákóczi Road (1955). 1-2: Grave R/225; 3-7: Grave R/266 

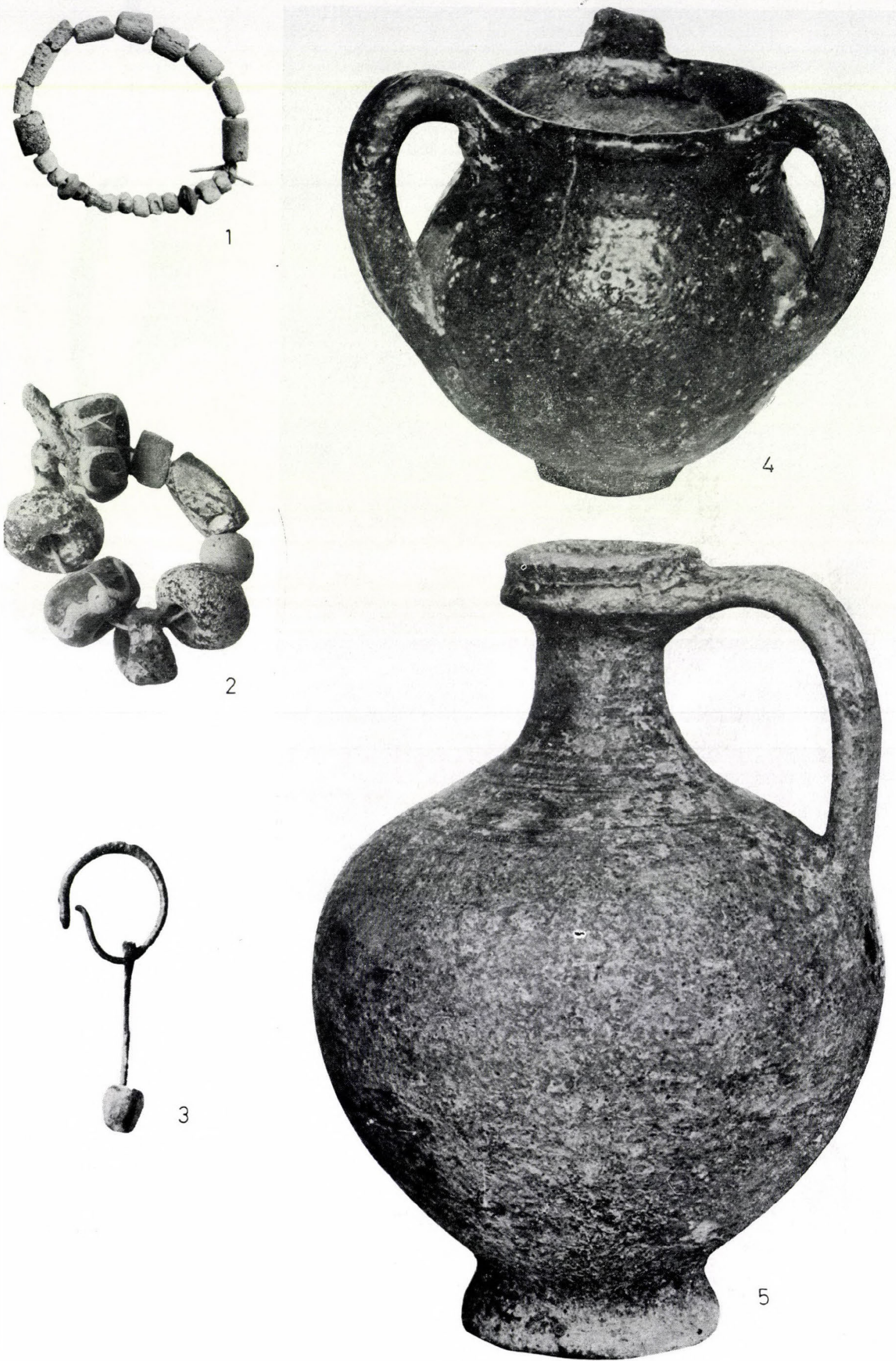

Plate 34 Regős Street (1940). Grave R/244 (scale $1: 1$ ) 

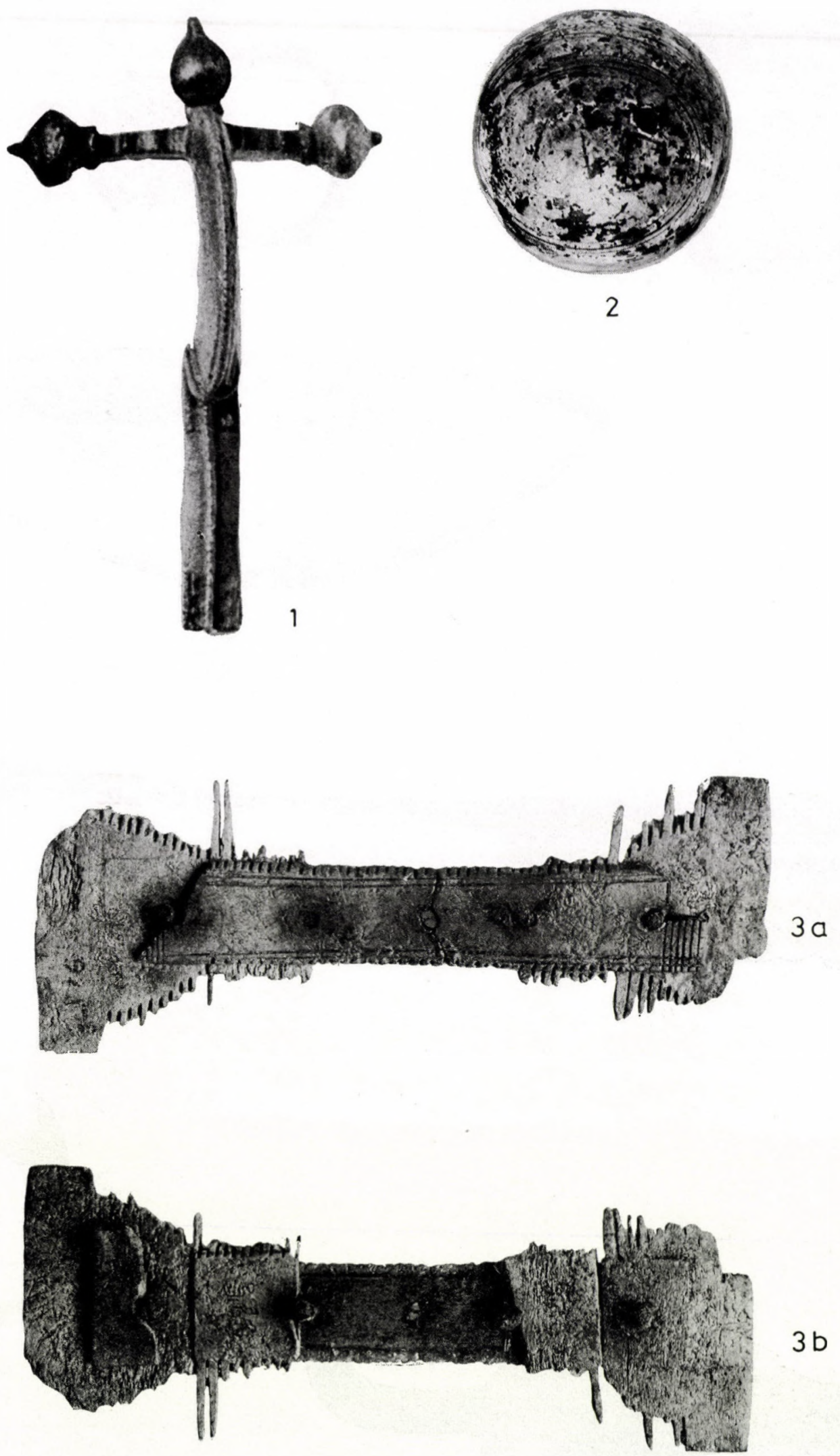

Plate 35 Anna Street (1927). 1: Grave R/260; 2-3: Grave R/261 (scale $1: 1$ ) 

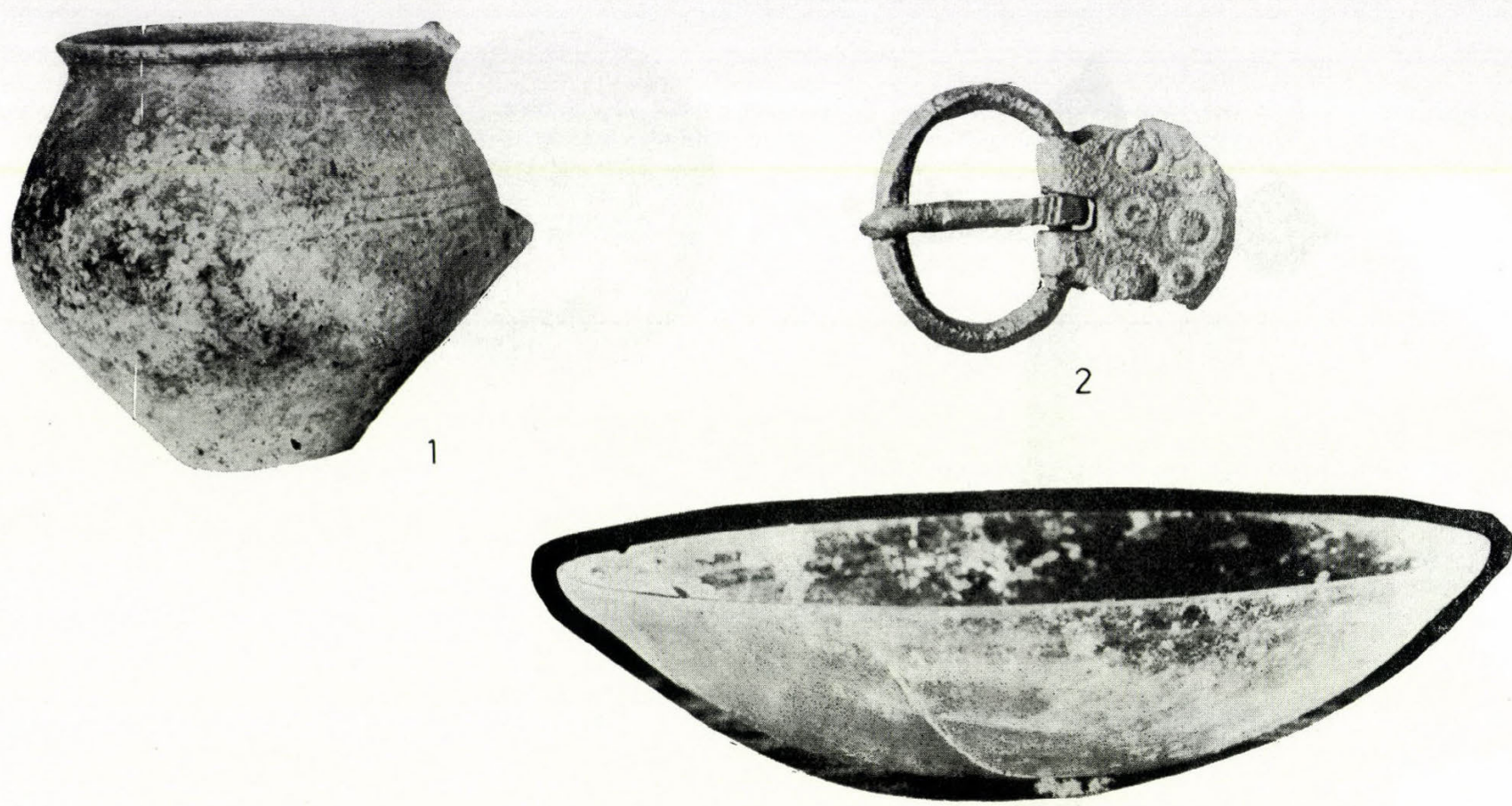

3
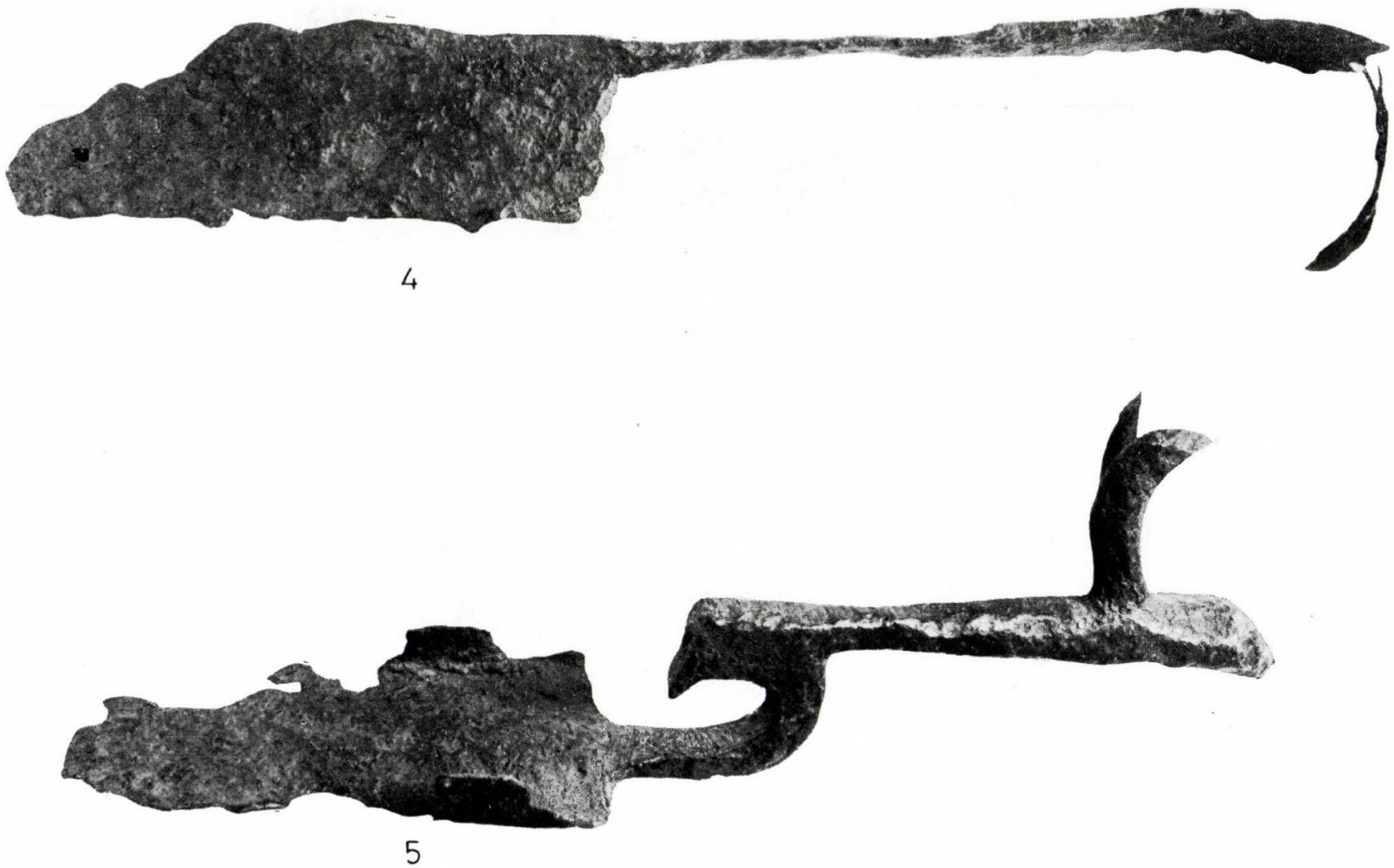

Plate 36 Rudas László (Gründler) Street (1941). 1: Grave R/263; 2-5: Grave R/264 

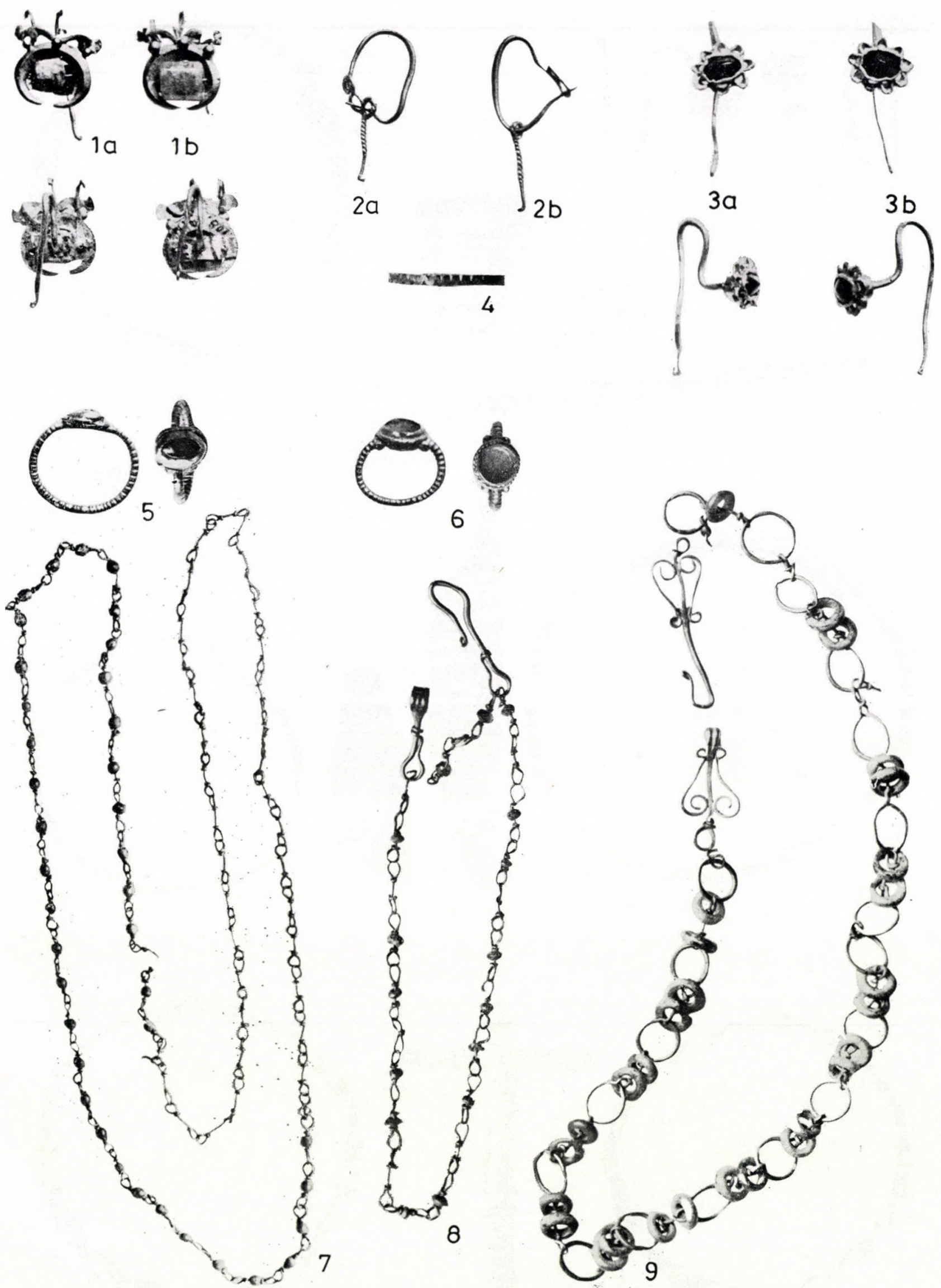

Plate 37 Gold jewels from unidentifiable graves; 5: Grave R/191 

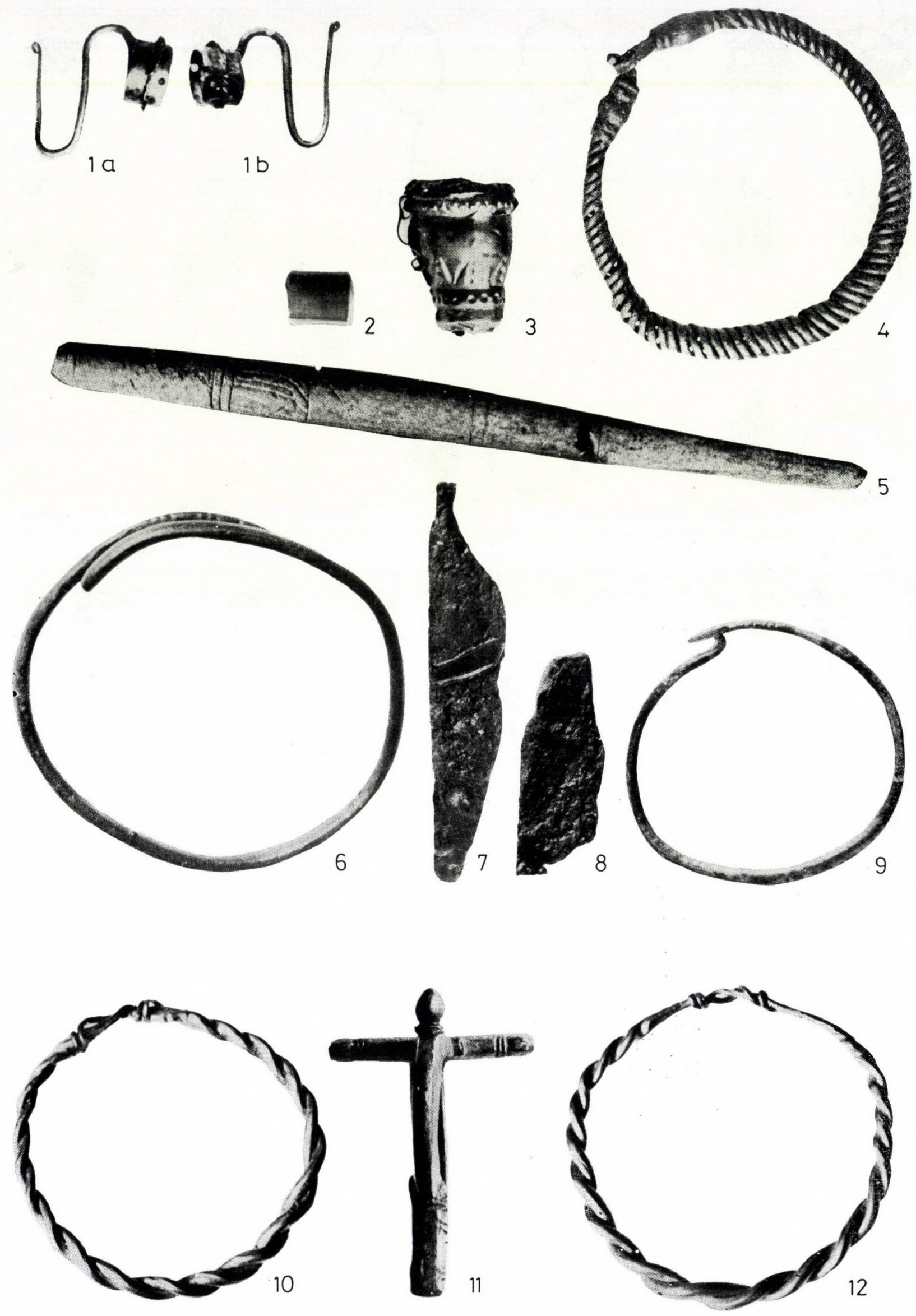

Plate 38 1-5: Grave R/275; 6: Grave R/277; 7-8: Grave R/280; 9: Graves R/291-292: 10: Grave R/290; 11: Grave R/293; 12: Grave R/290 

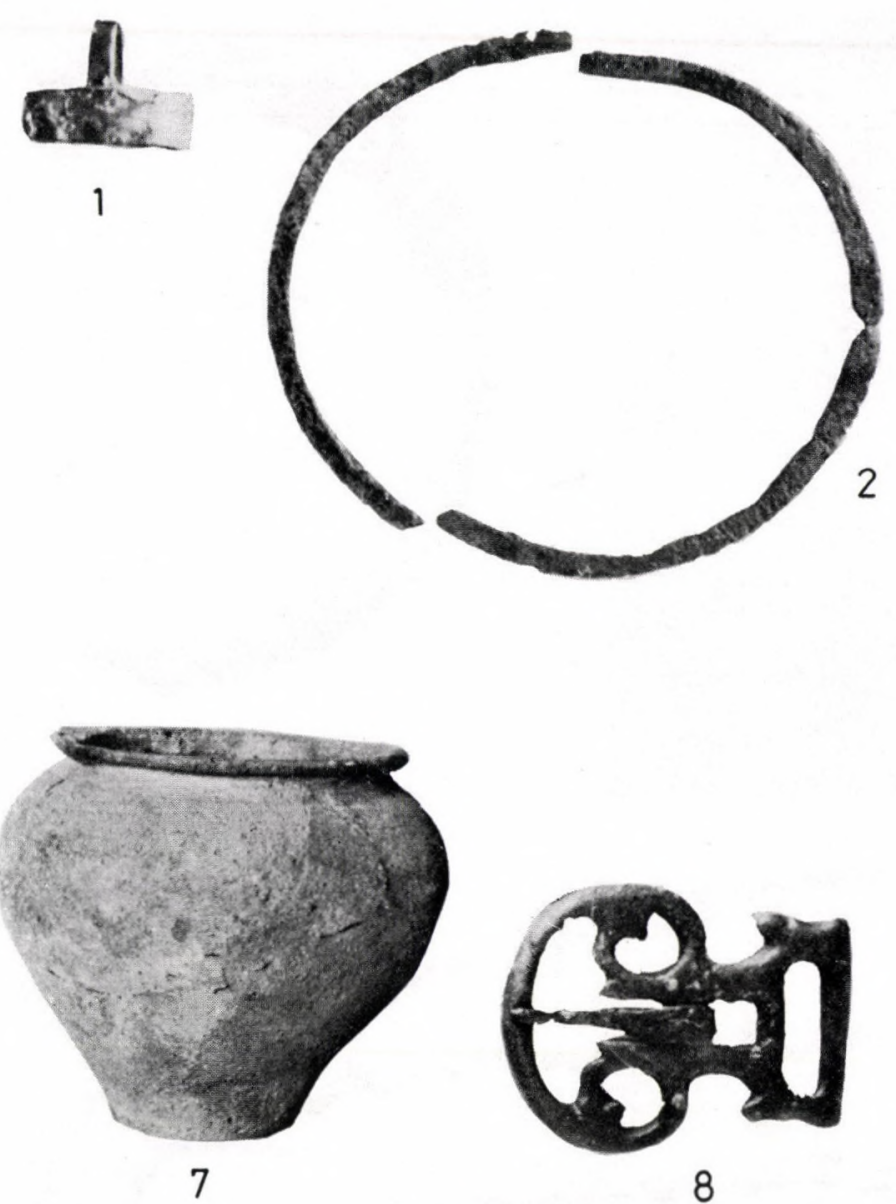

8

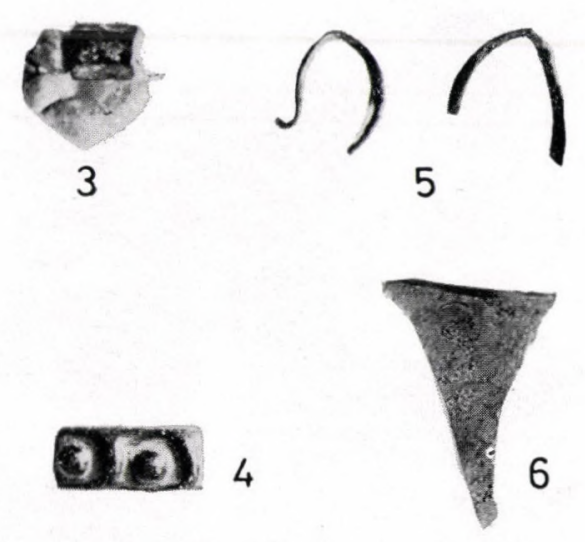

7
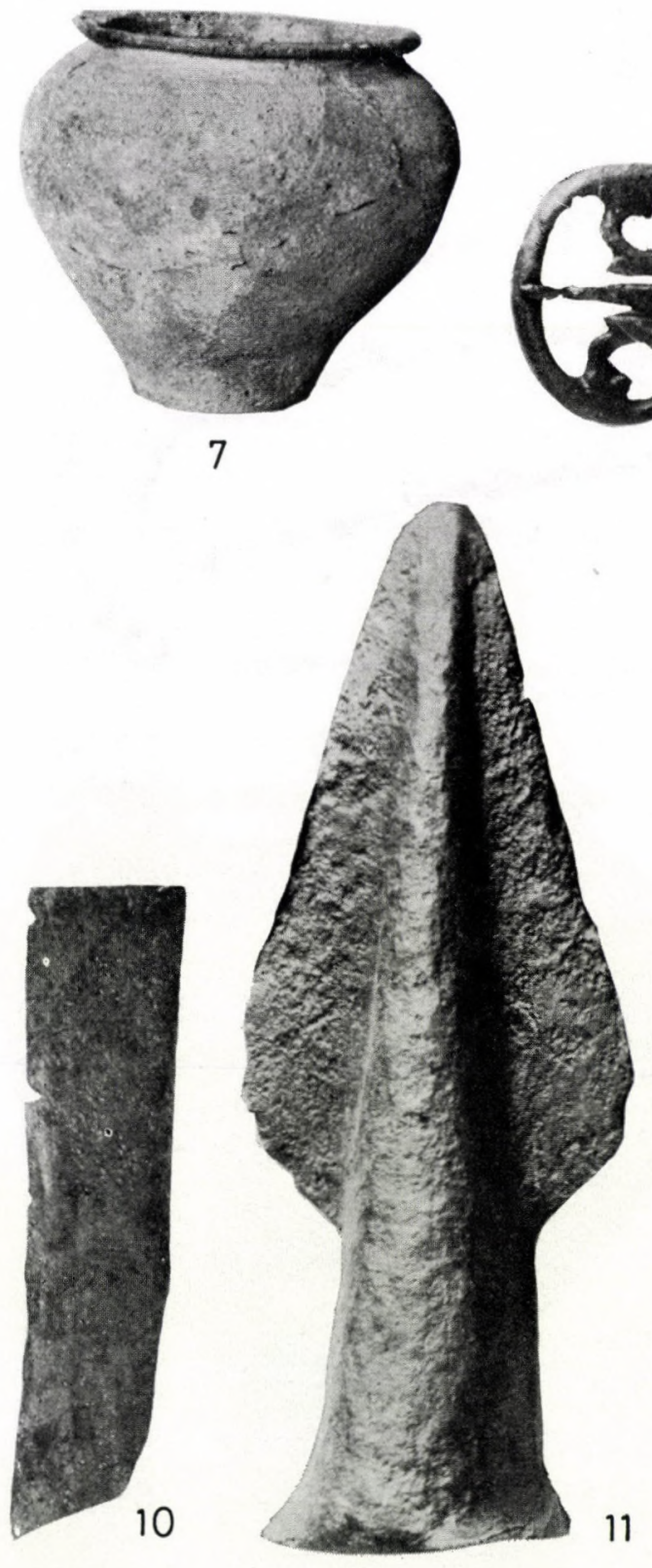

11
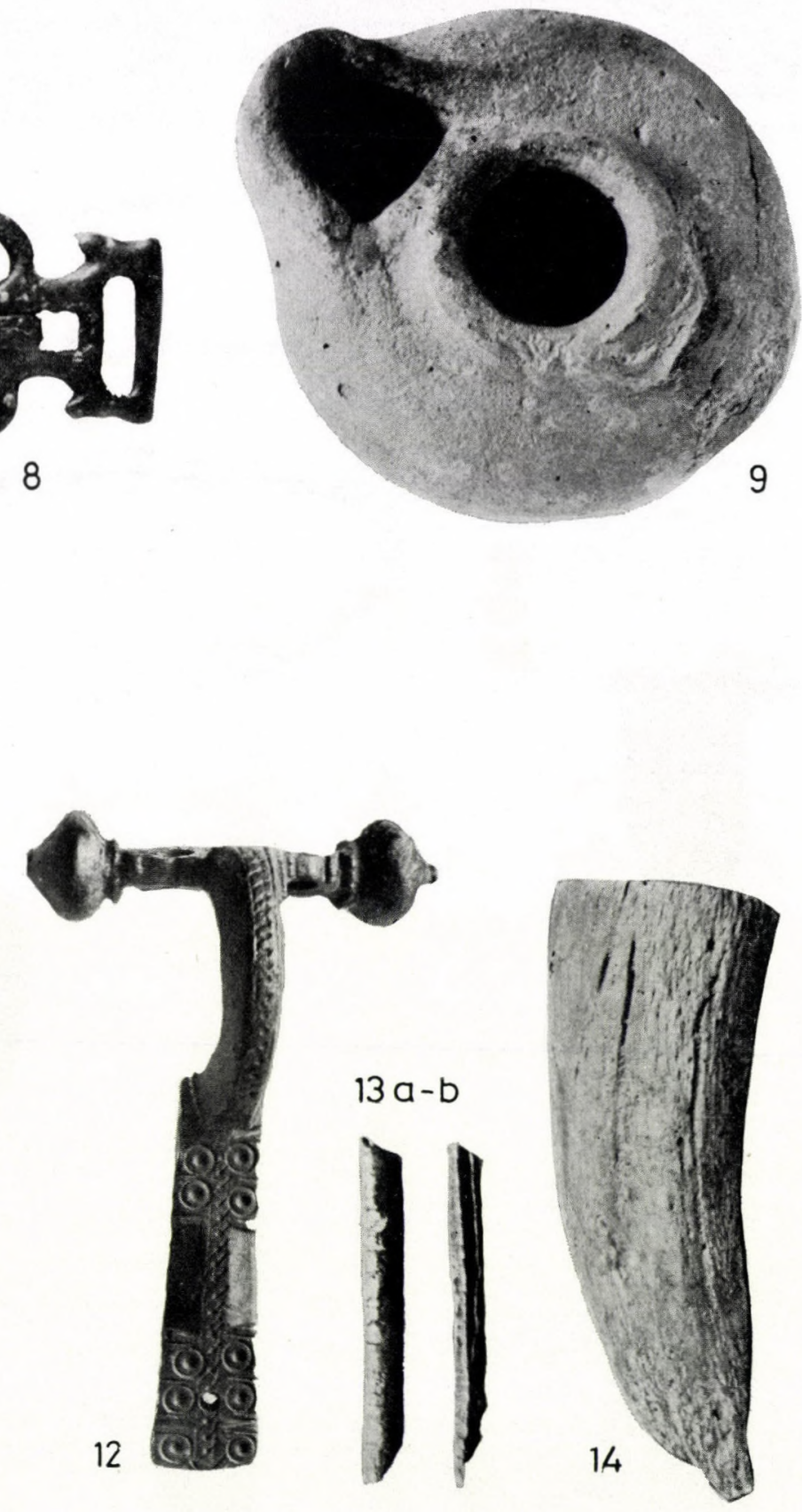

Plate 39 1-2: Grave R/295; 3-6: Grave R/307; 7: Rácváros; 8: Grave R/304; 9: Graves 307-308; 10-14: Grave R/308 

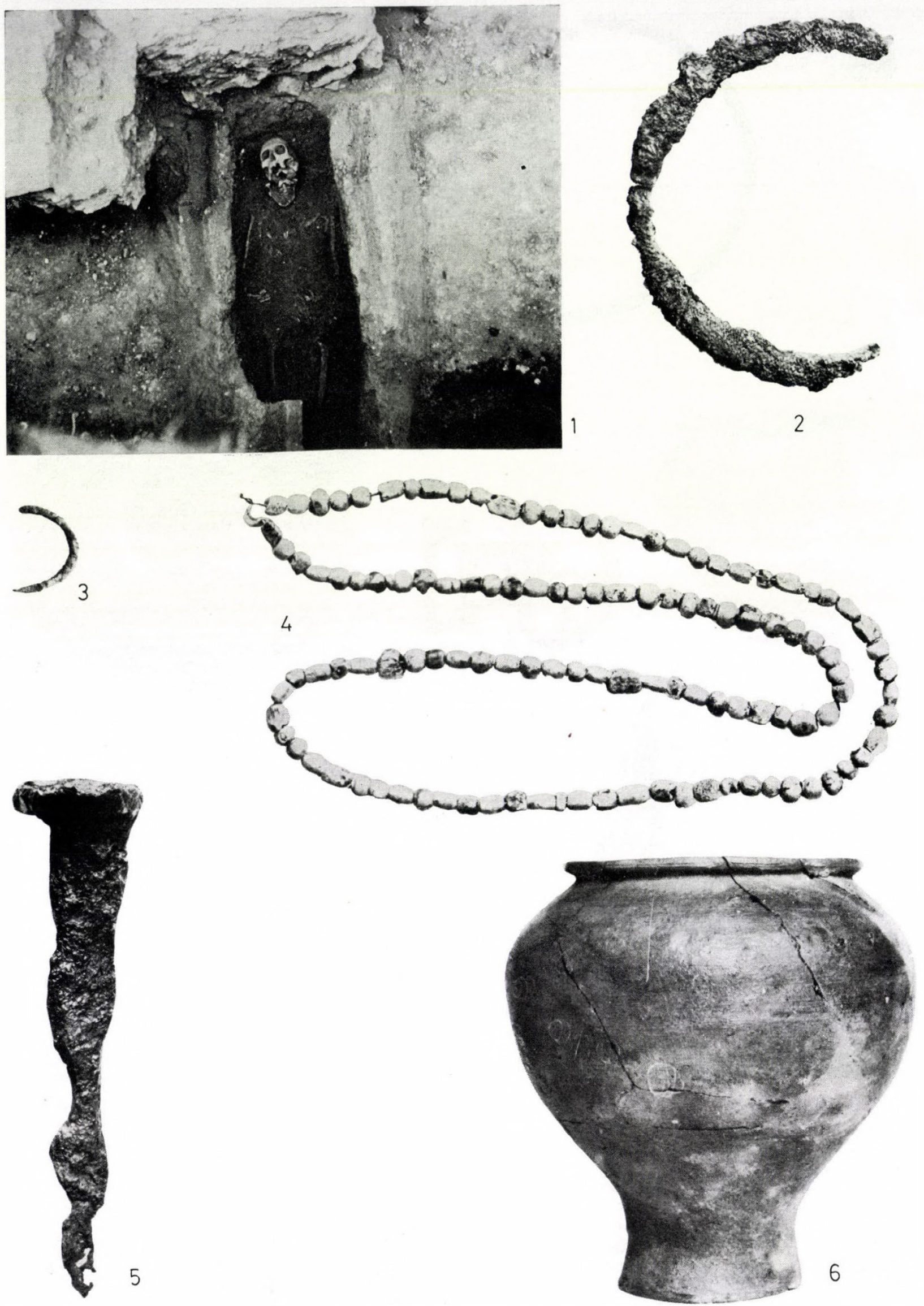

Plate 40 Courtyard of the Post Office (1964). 1-3: Grave R/318; 4-6: Grave R/321 


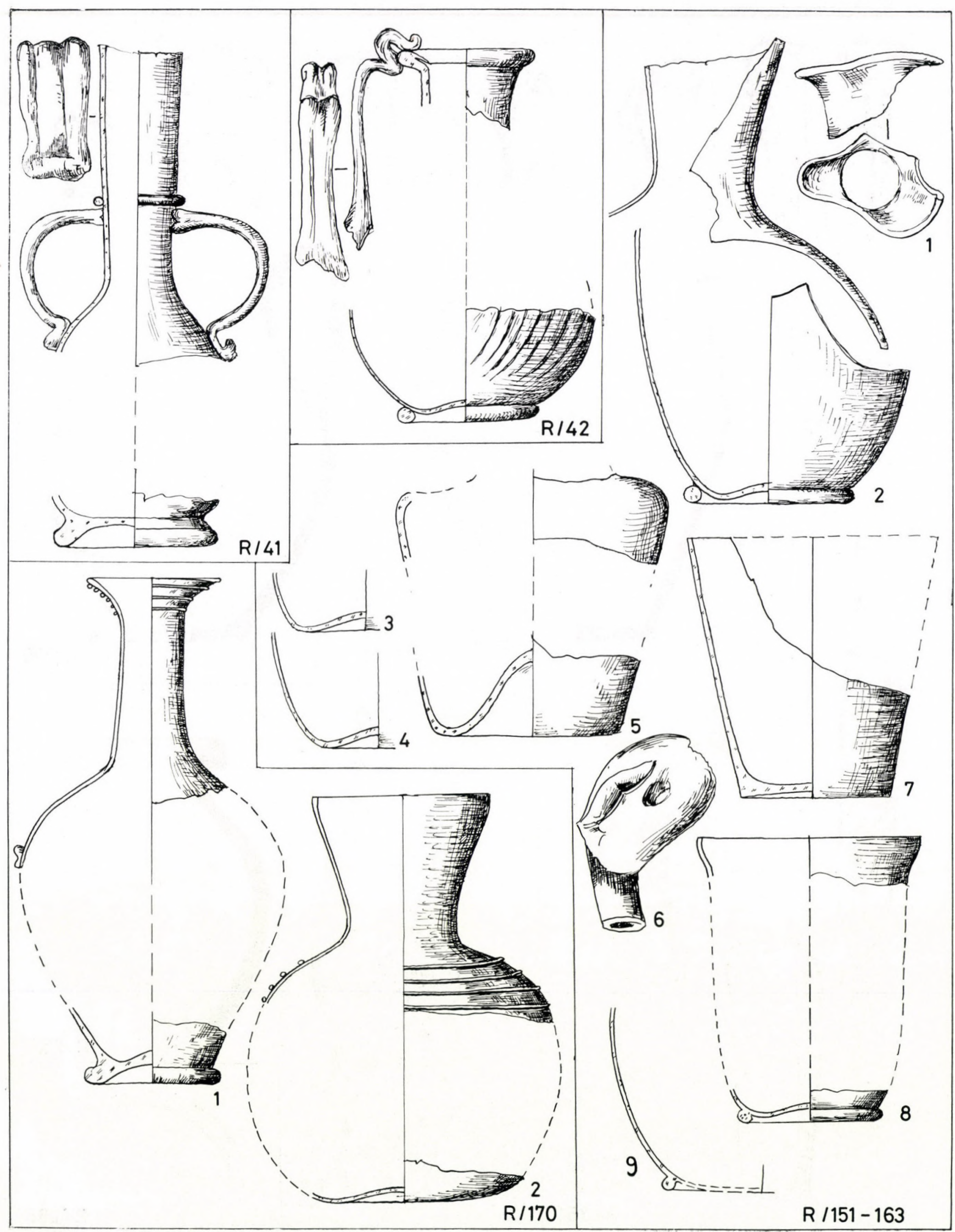

Plate 41 Glass-ware from Graves R/41-42, 151-163,170 


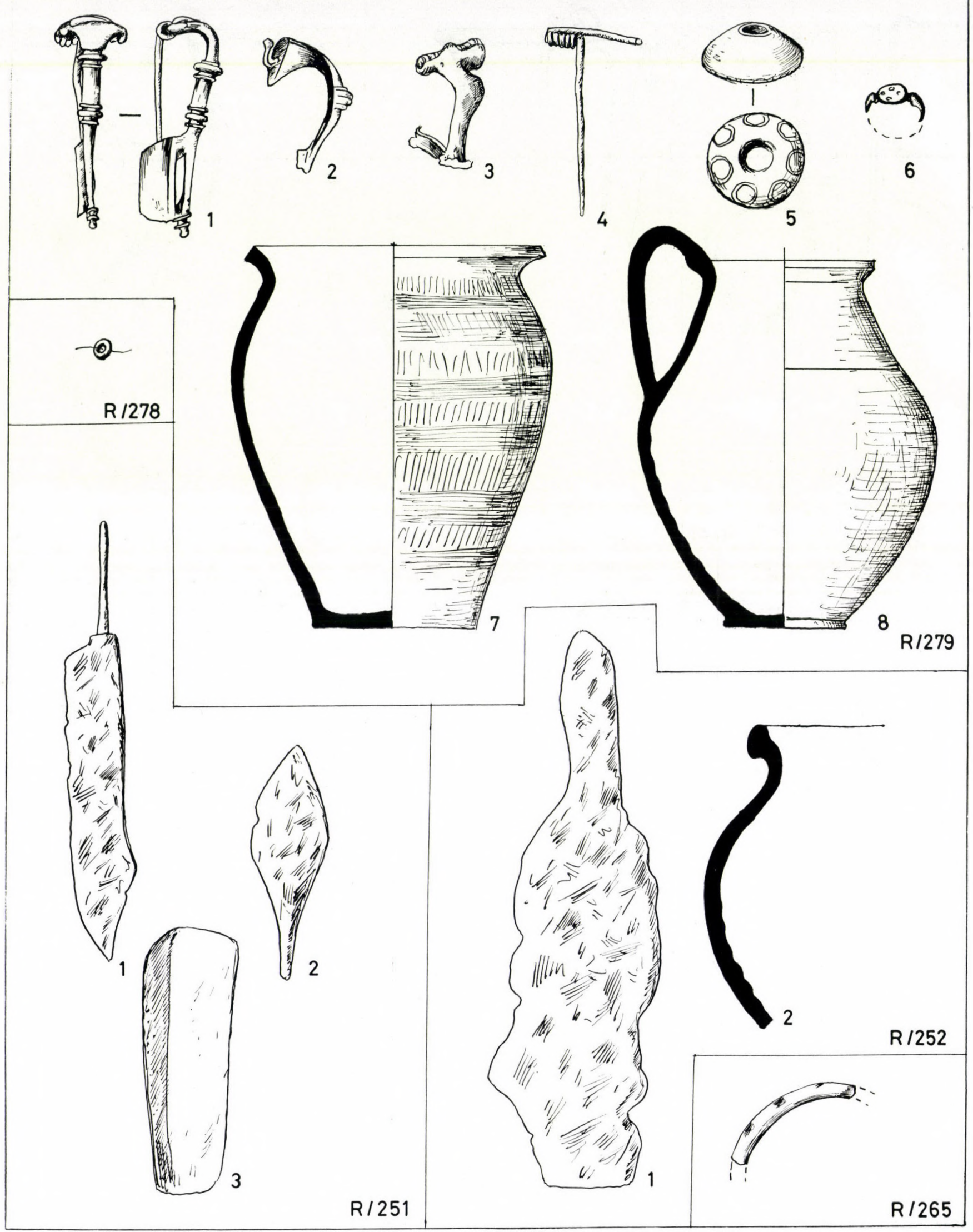

Plate 42 Graves R/251-252, 265, 278, 279 


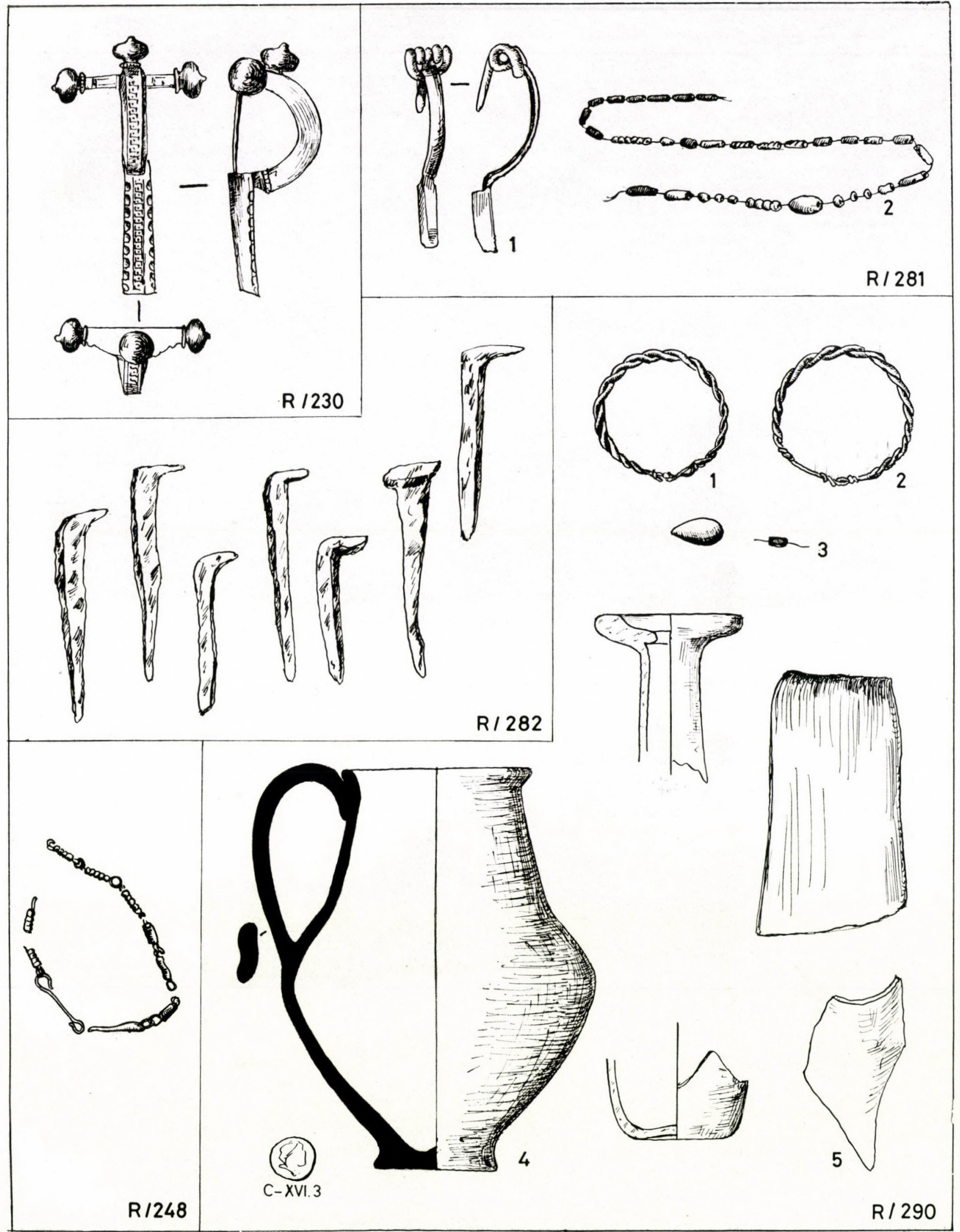

Plate 43 Graves R/230, 248, 281-282, 290 

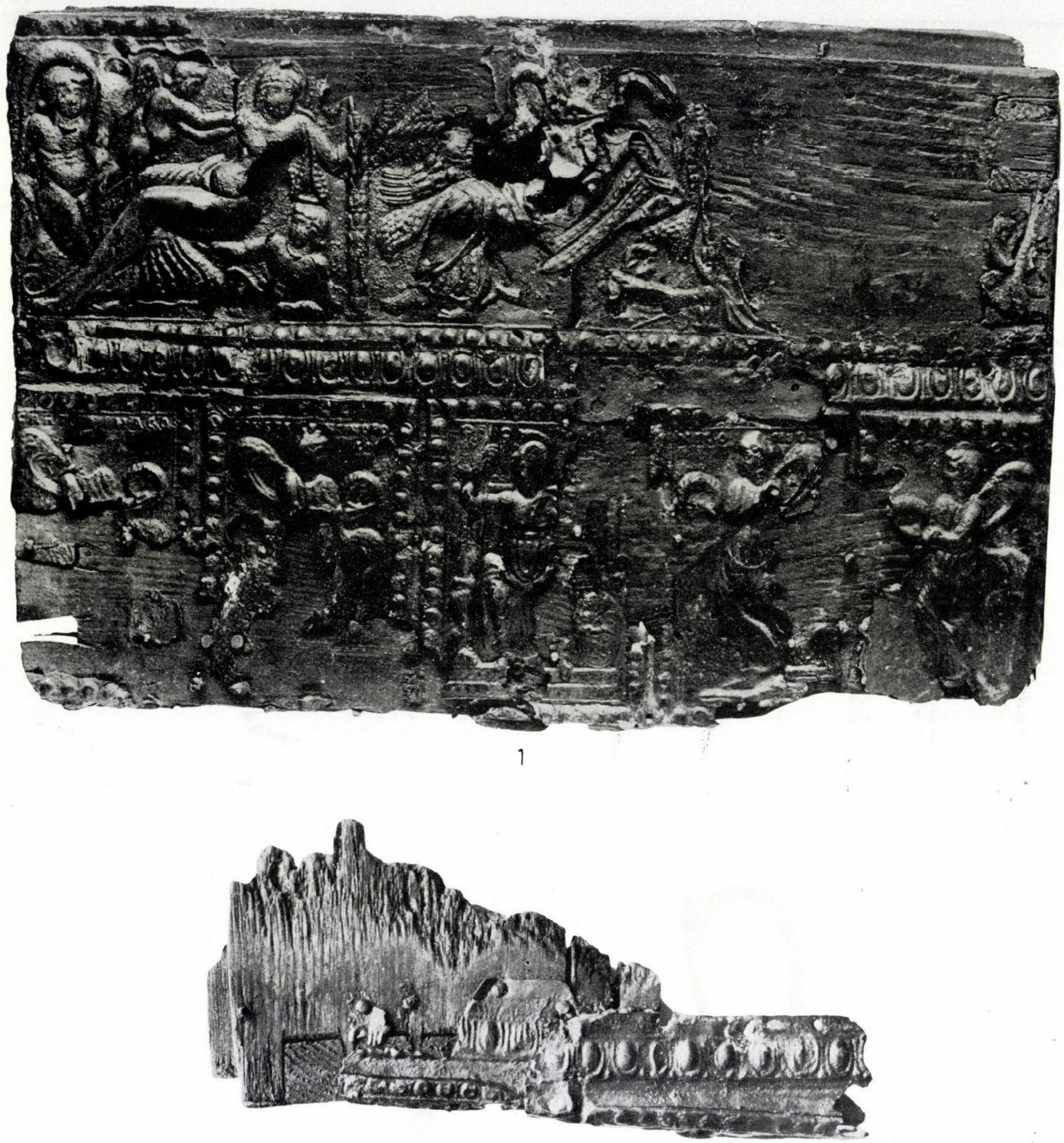

la

Plate 44 Grave R/54: wooden casket; 1a: fragment from the side of the same casket 


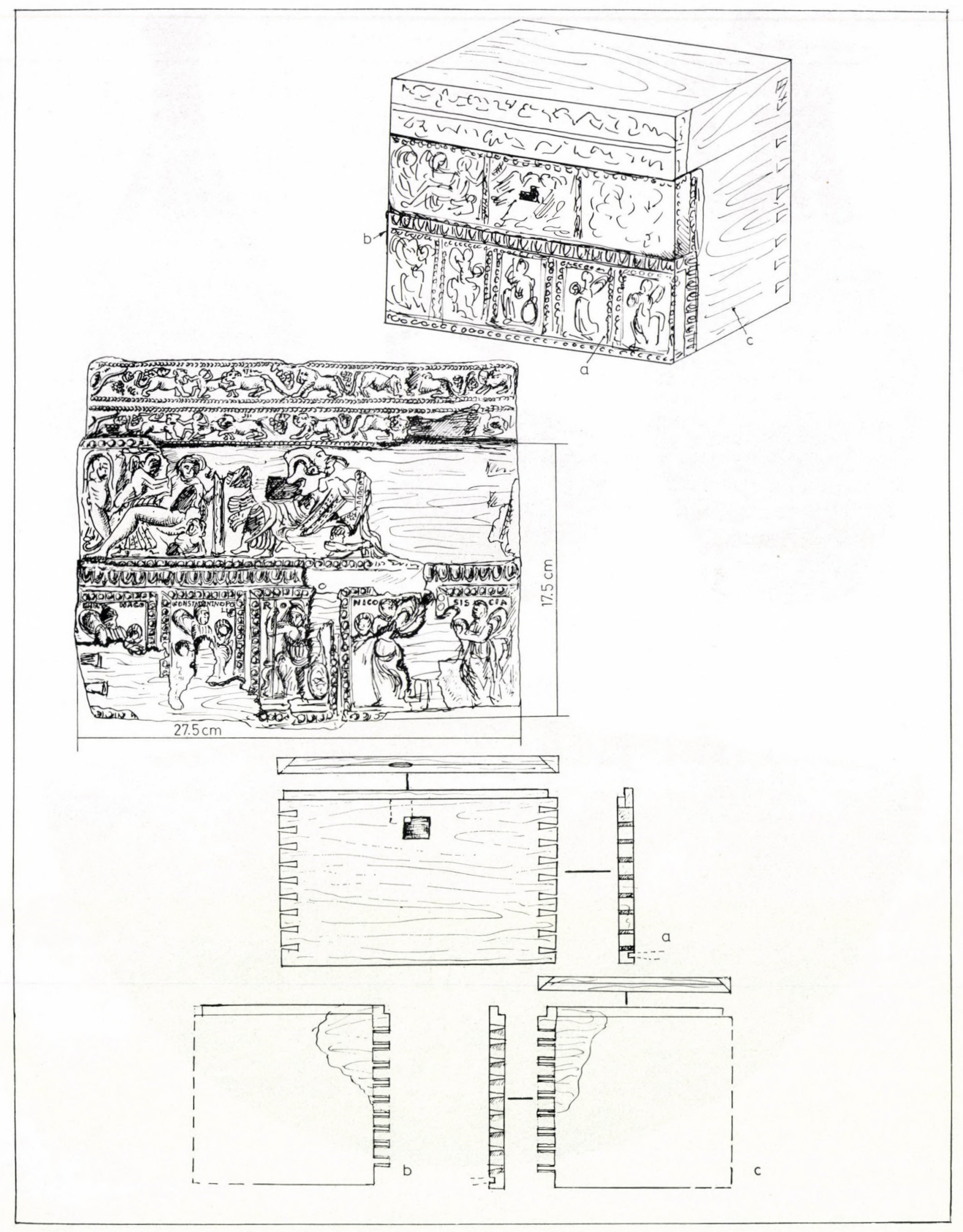

Plate 45 Grave R/54: reconstruction of the wooden casket 

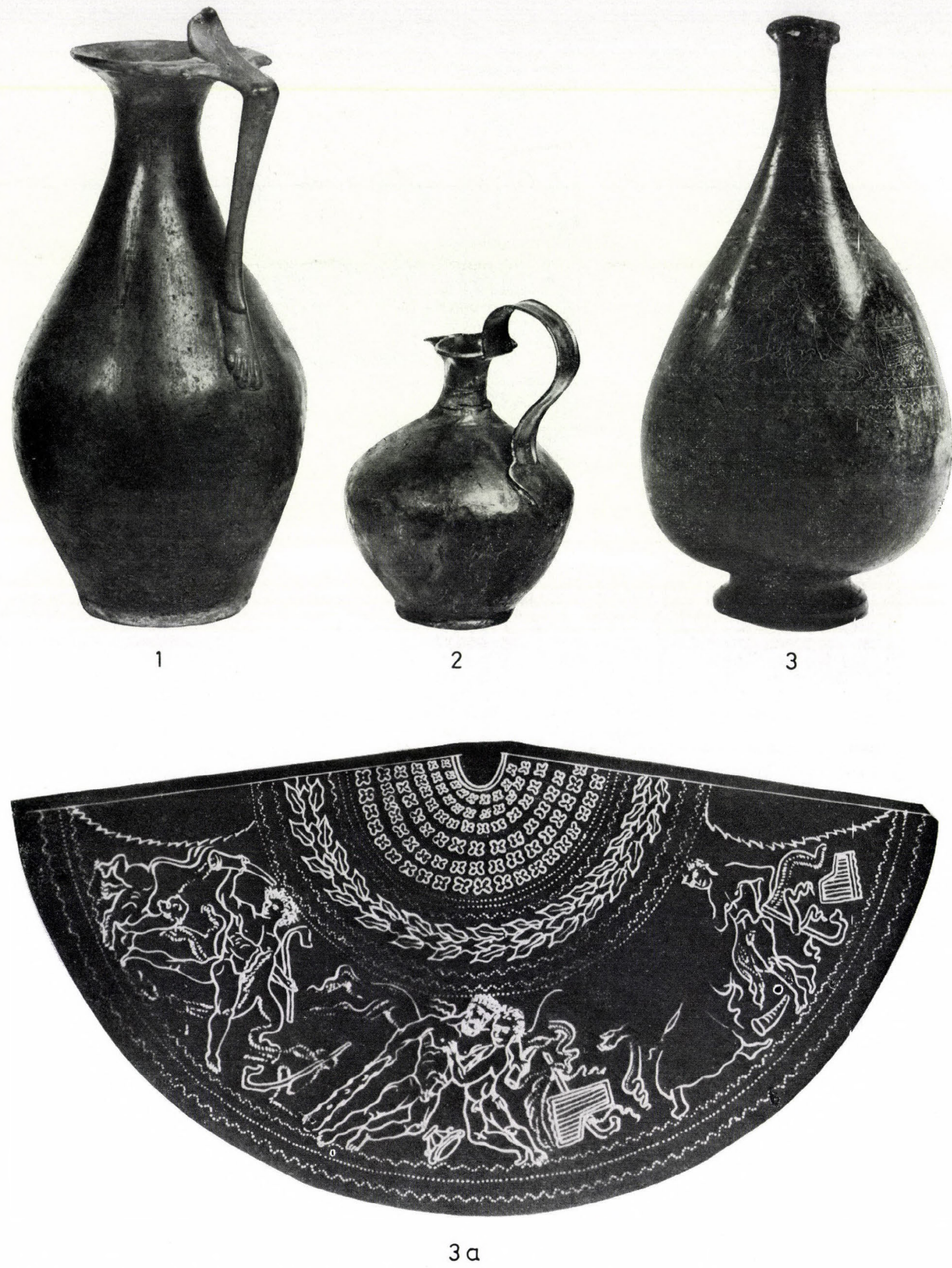

Plate 46 Homokbánya Lane (1899). 1-3: bronze vessels; 3a: stretched drawing of vessel 3 

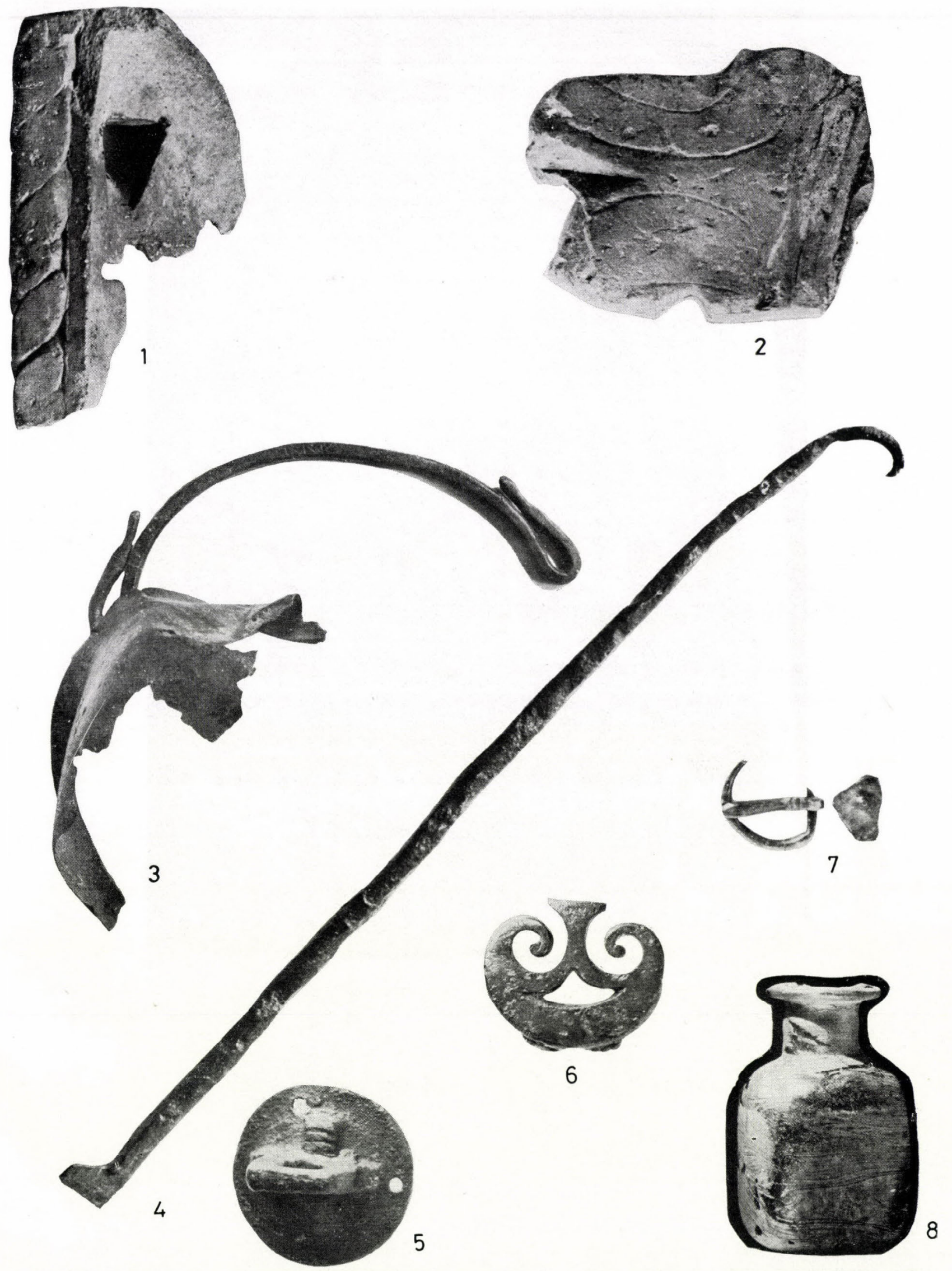

Plate 47 1: István Square; 2: Grave R/201; 3: Graves R/92-105; 4, 6: Kossuth Square; 5: 13, Széchenyi Square; 7: Grave R/180; 8: 28, Nagy Jenő Street 


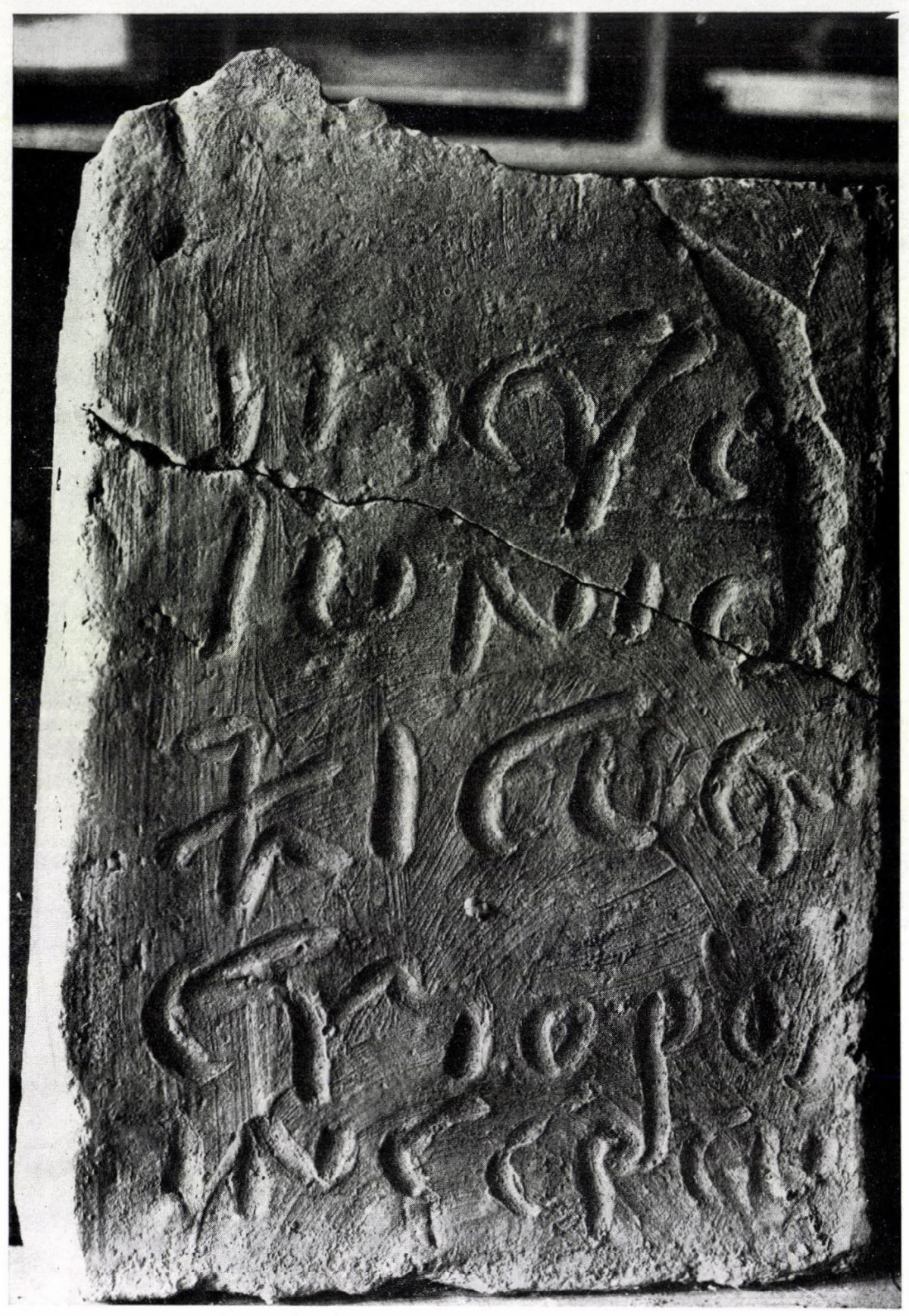

Plate 48 Brick with cursive inscription, Graves R/125-145 

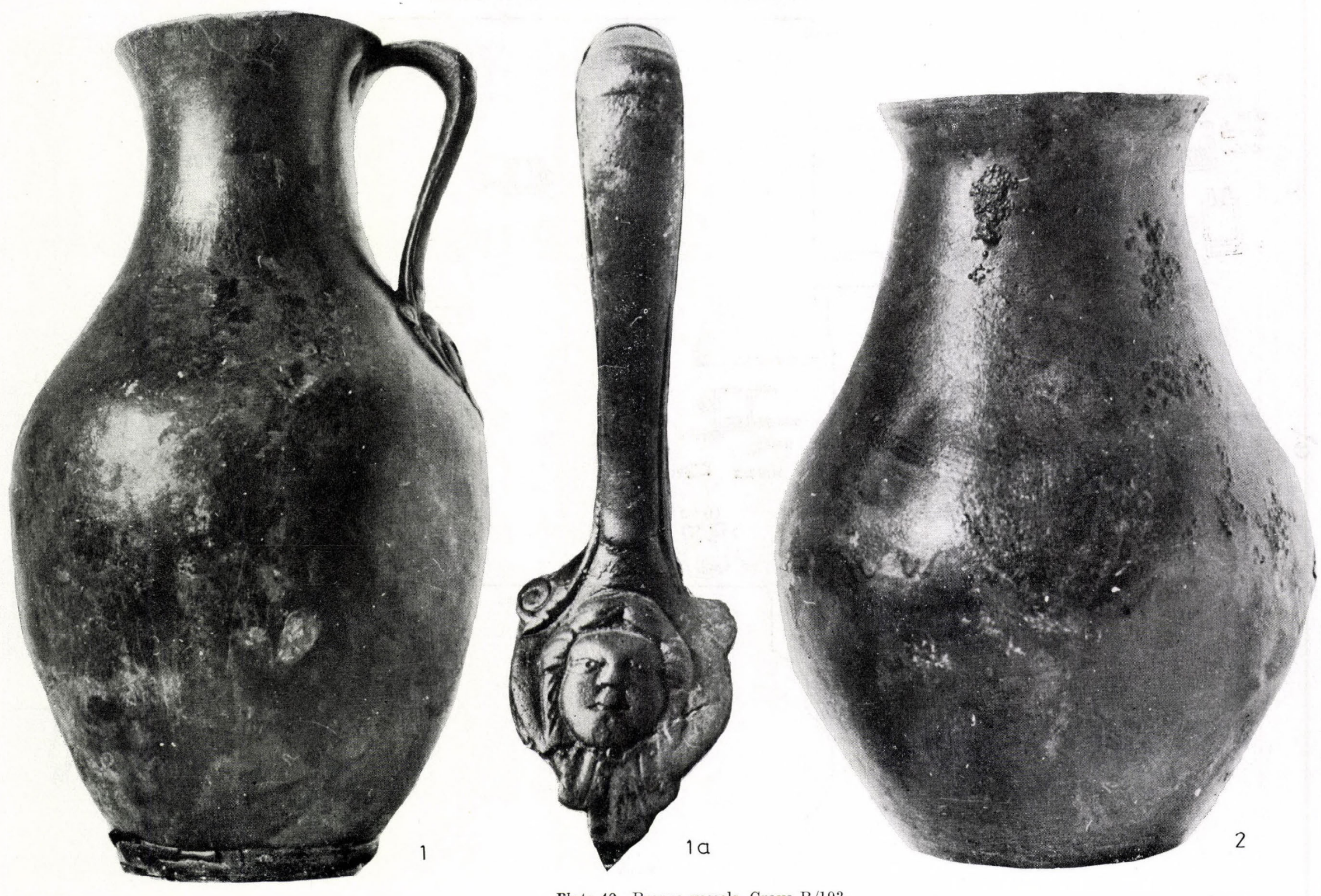

Plate 49 Bronze vessels, Grave R/193 


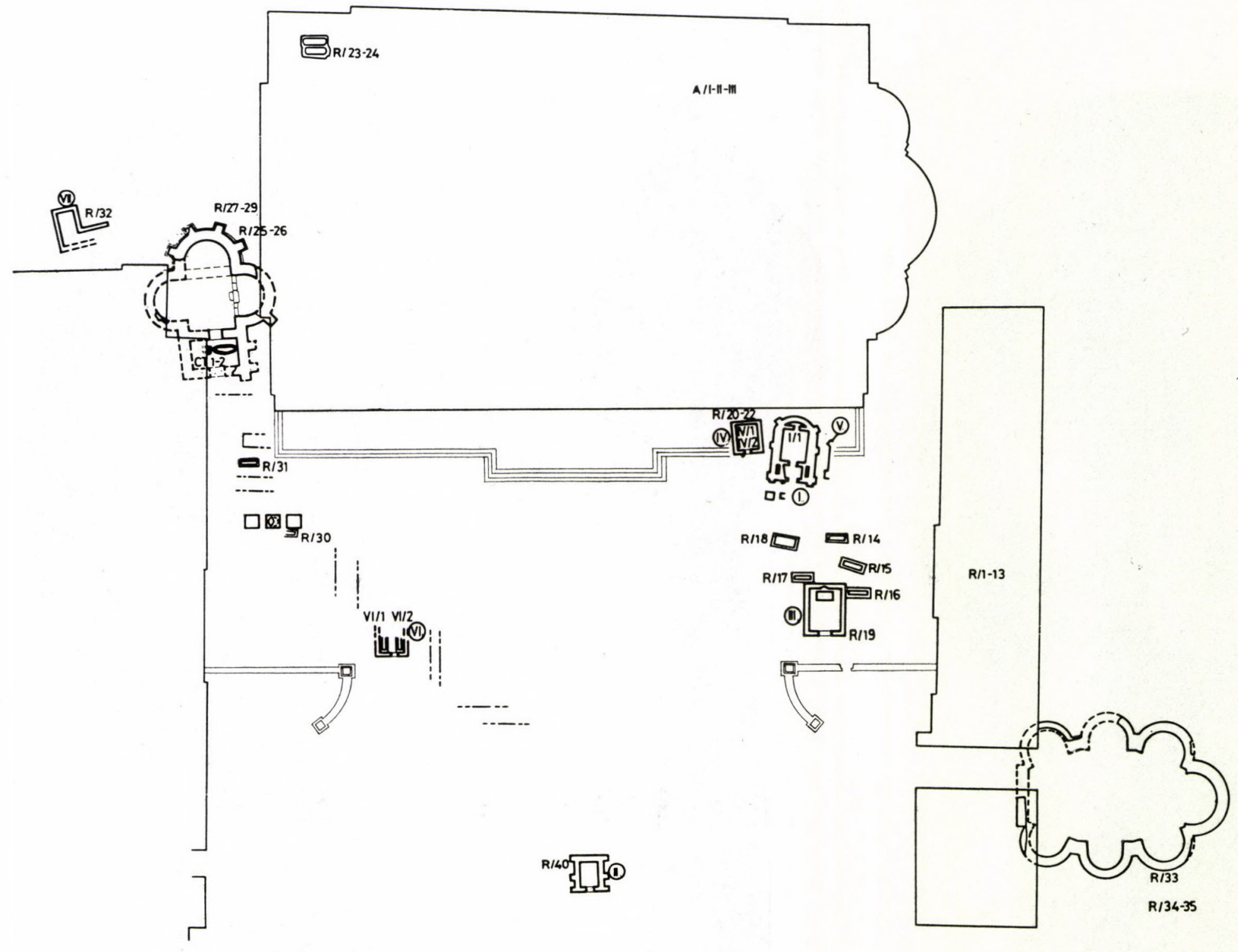

$\uparrow$

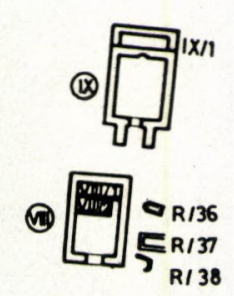




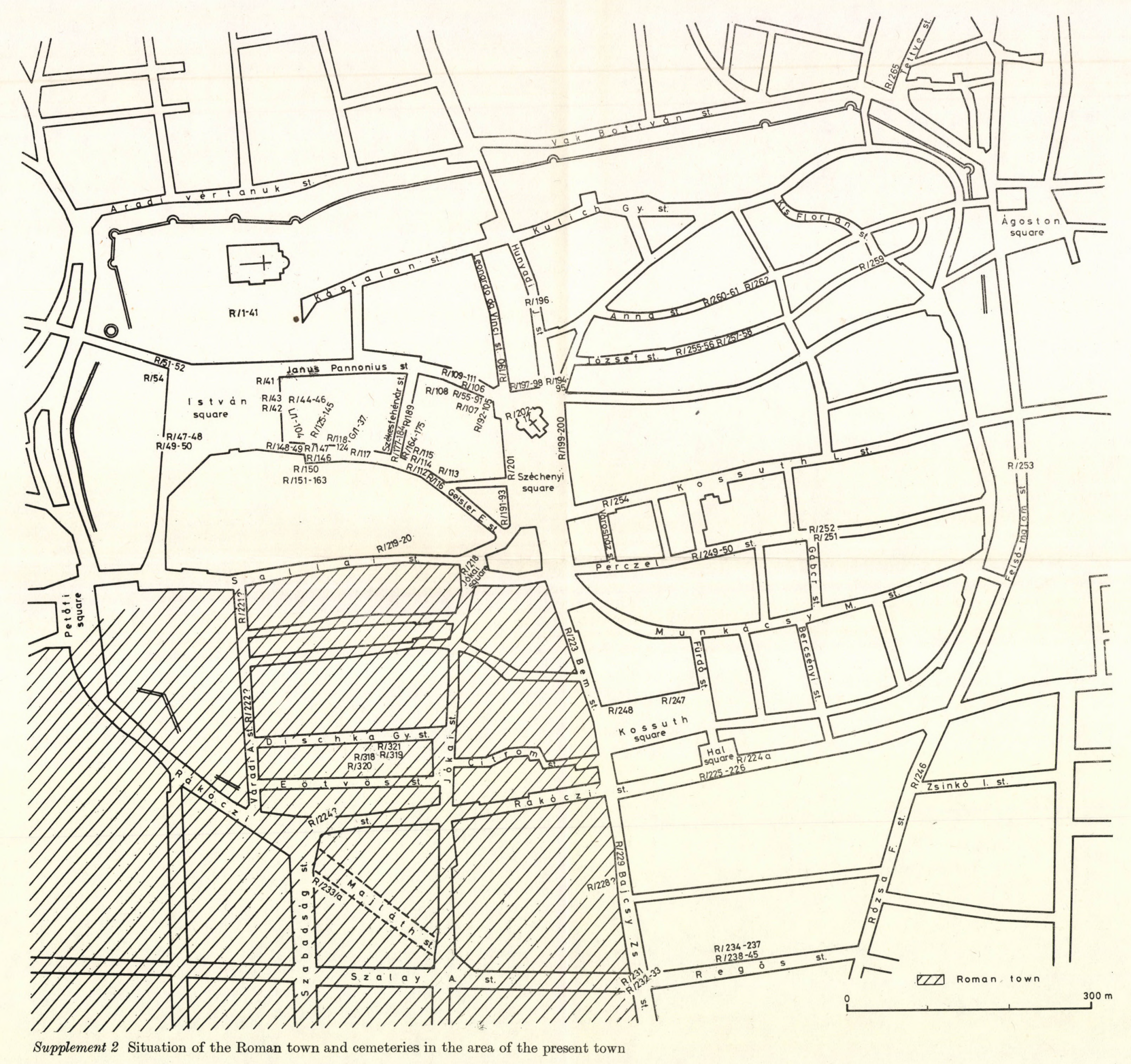




\section{.}




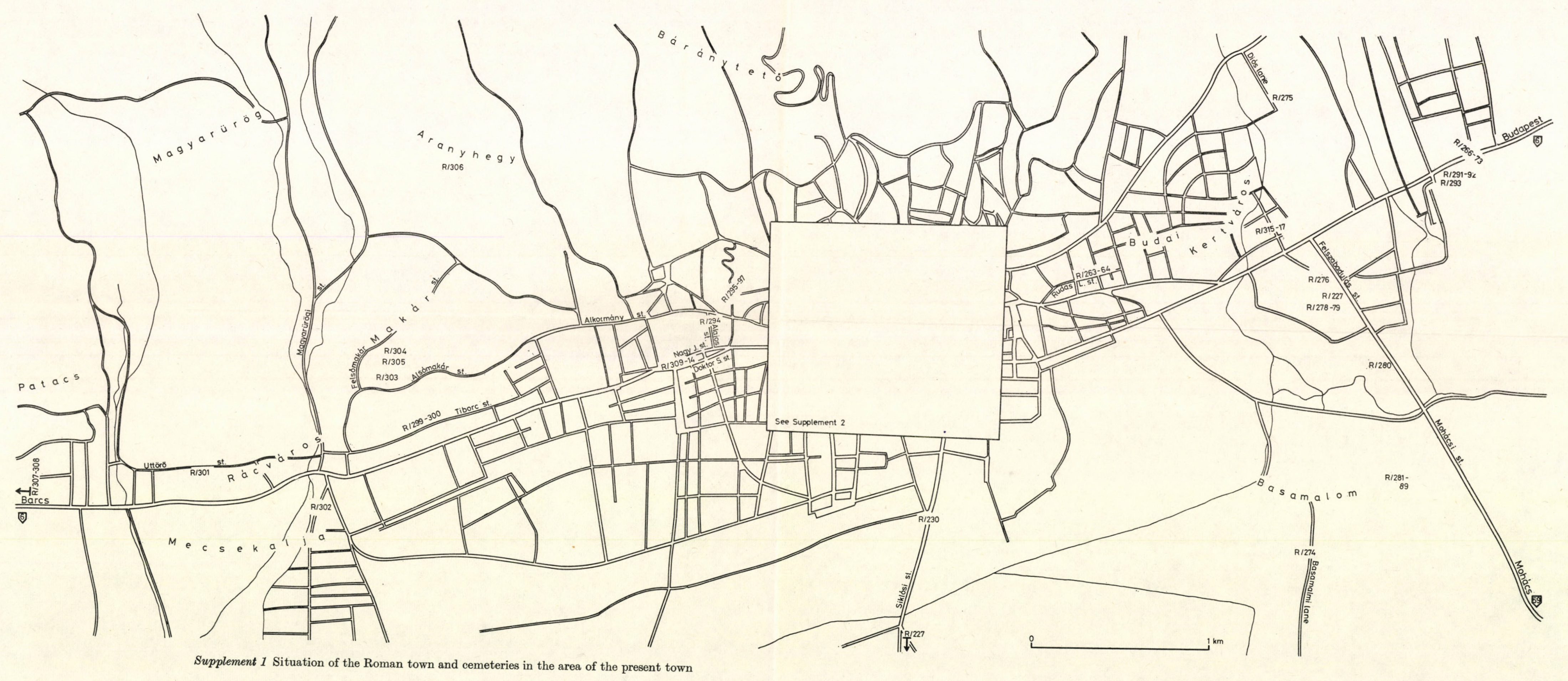



We recommend from the Series:

ROMAN MOSAICS IN HUNGARY

by Á. Kiss

In English • 72 pages • 47 photos • 1 supplement

$21 \times 29 \mathrm{~cm}$

MITTELBRONZEZEITLICHE TELL-SIEDLUNG BEI BÉKÉS

by J. Banner, I. Bóna

In German 156 pages • 53 photos • 2 supplements $21 \times 29 \mathrm{~cm}$

DAS BRONZEZEITLICHE GRÄBERFELD BEI TÁPÉ by 0 . Trogmayer

In German - 268 pages $\cdot 61+$ XXVII plates - Numerous figures 14 iables • 36 supp!ements $21 \times 29 \mathrm{~cm}$

Distributors:

KULTURA

H-1389 Budapest, P.O.B. 149 


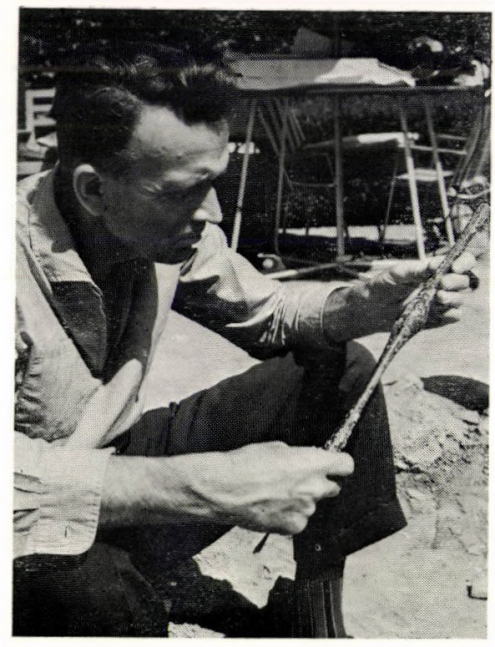

Ferenc Fülep graduated at Debrecen University in 1946. From 1947 to 1949 he was Director of the Museum of County Fejér and Székesfehérvár, in 1951 he became Director General of the Hungarian National Museum, Budapest, and between 1953 and 1956 he also held the position of Head of the Department of Museums in the Ministry of Culture. In 1972 he was granted the degree Dr. Sc. Between 1949 and 1957 he was excavating the Roman camp of Campona, and from 1954 he has carried out archaeological excavations in Pécs, the Roman Sopianae, in southern Hungary. He is Editor-in-Chief of Archaeologiai Értesitő and also of Folia Archaeologica, the annual of the Hungarian National Museum.

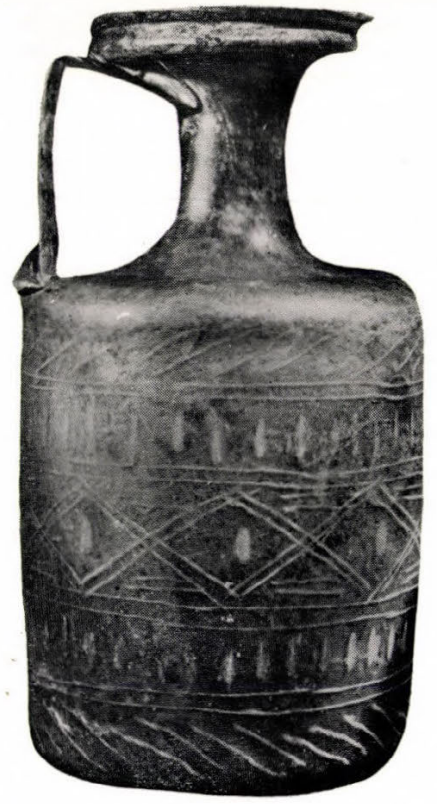

